\title{
Structure of Hot Molecular Cores
}

\author{
In a u g u r a 1-Dis sertation \\ zur \\ Erlangung des Doktorgrades \\ der Mathematisch-Naturwissenschaftlichen Fakultät \\ der Universität zu Köln \\ vorgelegt von \\ Rainer Rolffs \\ aus Bonn
}


Berichterstatter: Prof. Dr. Peter Schilke (Gutachter)

Prof. Dr. Karl Menten

Tag der mündlichen Prüfung: 22. 6. 2011 


\section{Contents}

1 Introduction $\quad 9$

1.1 Pressure in the Interstellar Medium . . . . . . . . . . . . . . . . . . . . . . . . . . . . . . . . . . . . .

1.1.1 Heating and Cooling .......................... 9

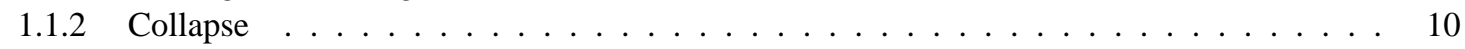

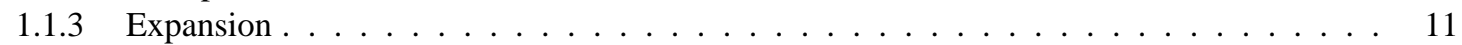

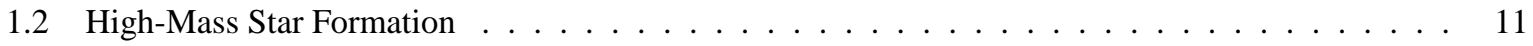

$1.2 .1 \quad$ Impact $\ldots \ldots \ldots \ldots \ldots \ldots \ldots \ldots$

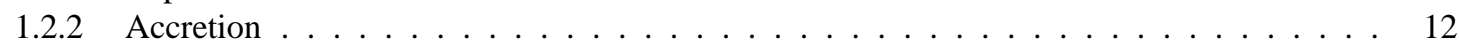

1.2 .3 An Evolutionary Sequence . . . . . . . . . . . . . . . . . . . . . . 13

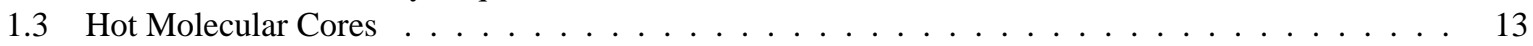

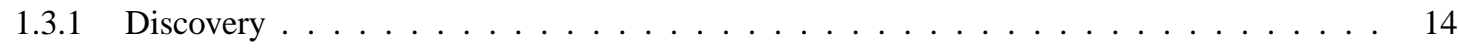

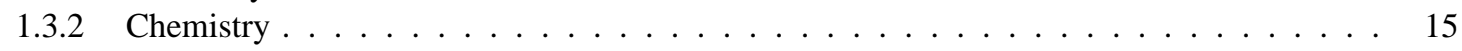

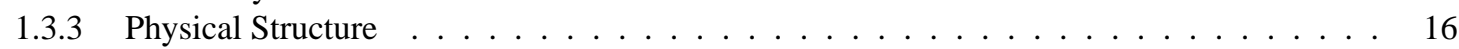

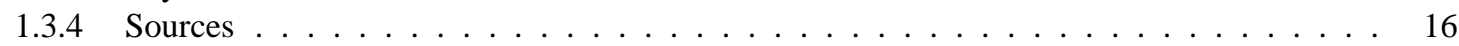

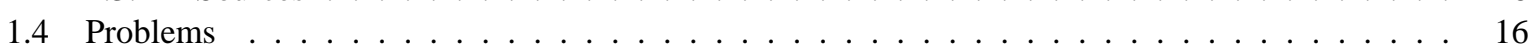

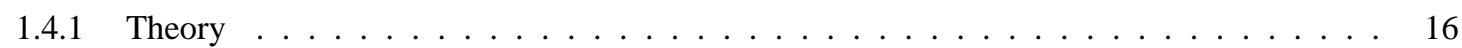

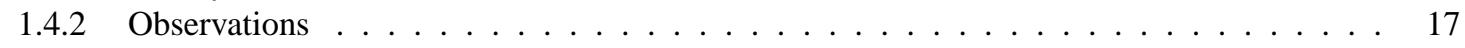

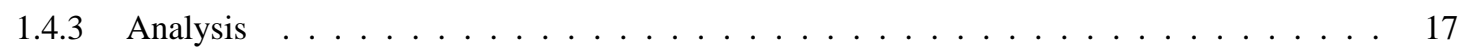

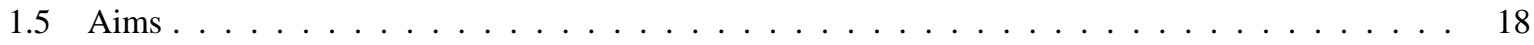

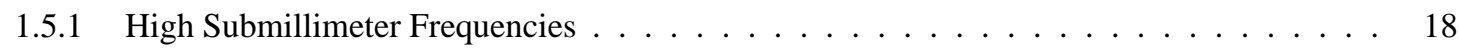

1.5 .2 High Resolution . . . . . . . . . . . . . . . . . . . . . . . . . . . . . . . . . . . . . . . .

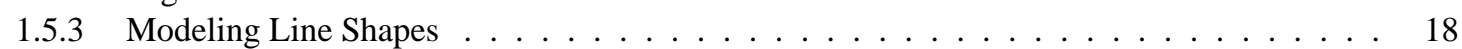

1.5.4 Three-dimensional Modeling . . . . . . . . . . . . . . . . . . 18

2 Radiative Transfer $\quad 19$

2.1 Molecular Energy Levels . . . . . . . . . . . . . . . . . . . . . . . . . . . 19

2.1 .1 Rotational Levels . . . . . . . . . . . . . . . . . . . . . . . . . 19

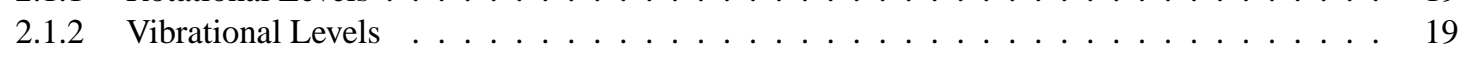

2.1 .3 Example: HCN Levels . . . . . . . . . . . . . . . . . . . . . . 20

2.2 Radiative Transitions . . . . . . . . . . . . . . . . . . . . . . . . . . . 20

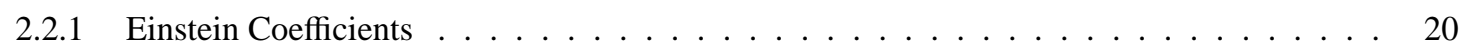

2.2 .2 Example: HCN Transitions . . . . . . . . . . . . . . . . . . . . . . 20

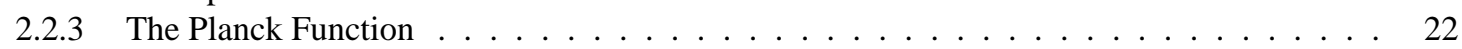

2.3 Level Populations . . . . . . . . . . . . . . . . . . . . . . . . . . . . 22

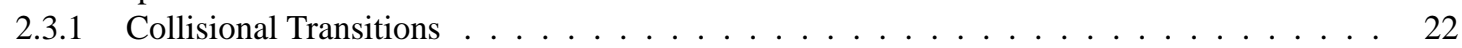

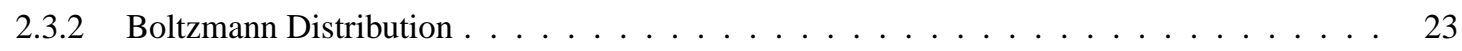

2.3.3 Local Thermodynamic Equilibrium (LTE) and Beyond . . . . . . . . . . . . . . . 23

2.3 .4 Non-LTE Methods . . . . . . . . . . . . . . . . . . . . . . . . . . . . . . . . . . . . . . . . . . . . .

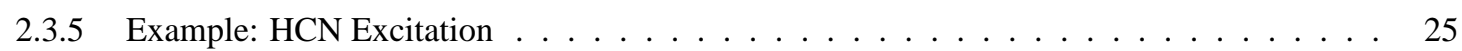

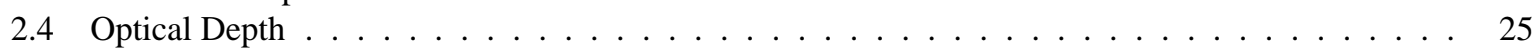

2.4 Basic Radiative Transfer . . . . . . . . . . . . . . . . . . . . . 25

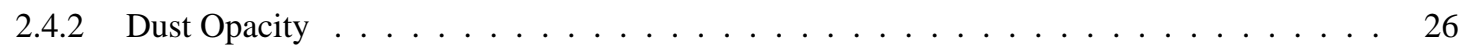

2.4 .3 Line Width . . . . . . . . . . . . . . . . . . . . . . 27

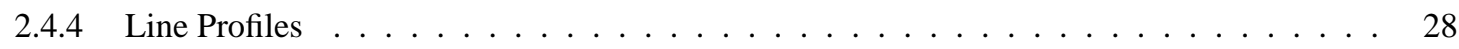

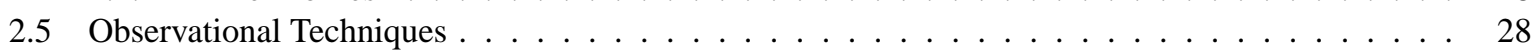

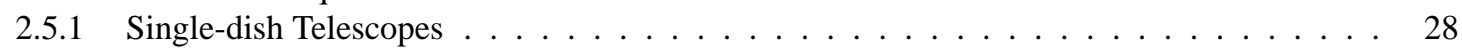

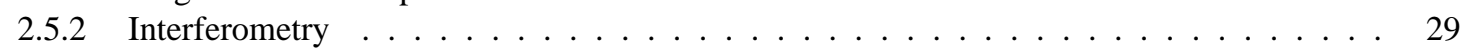




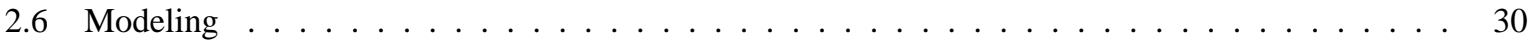

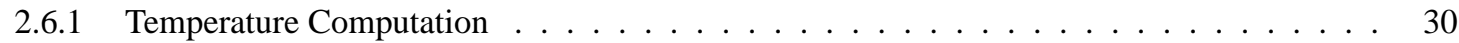

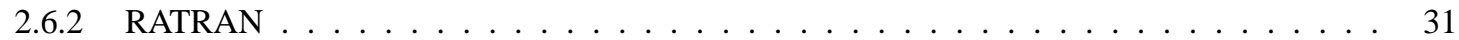

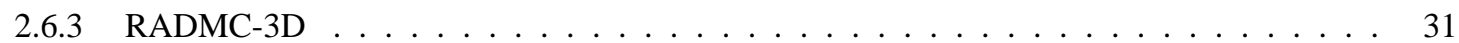

2.6 .4 Defining Parameters . . . . . . . . . . . . . . . . . . . . . . 31

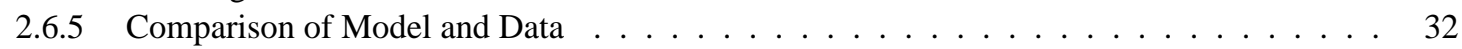

2.6 .6 Searching the Parameter Space . . . . . . . . . . . . . . . . . . . . . . 32

3 APEX paper: Structure of evolved cluster-forming regions 3

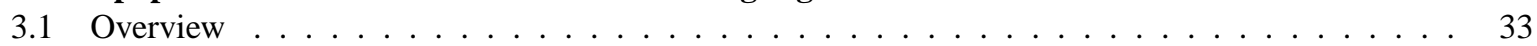

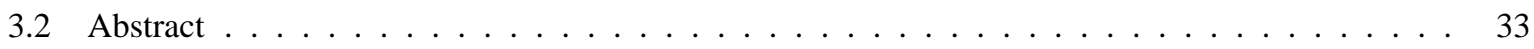

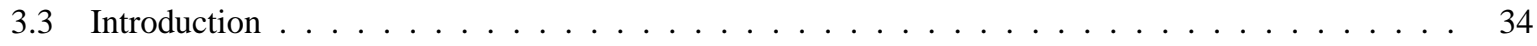

3.4 Observations and data reduction $\ldots \ldots \ldots \ldots \ldots \ldots \ldots \ldots \ldots$

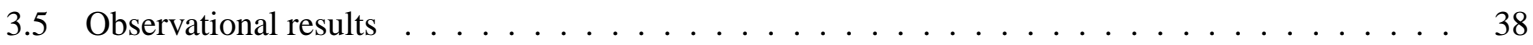

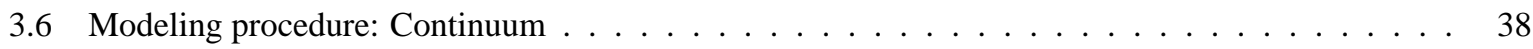

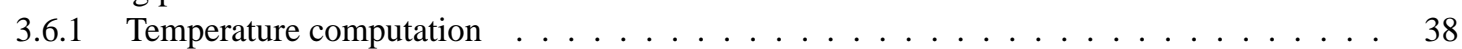

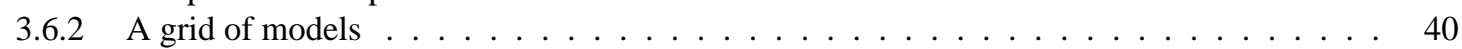

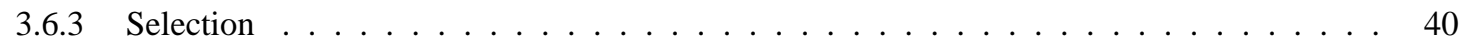

3.7 Modeling procedure: Lines . . . . . . . . . . . . . . . . . . . . . . . . 40

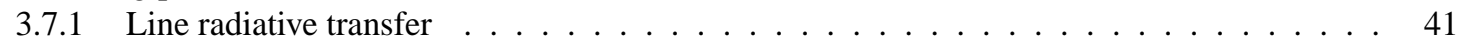

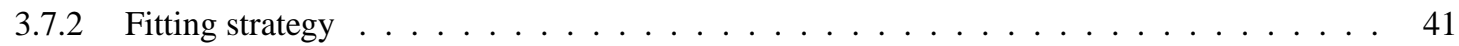

3.7 .3 The 'final' model . . . . . . . . . . . . . . . . . . . . . . . . 42

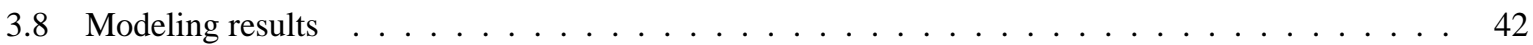

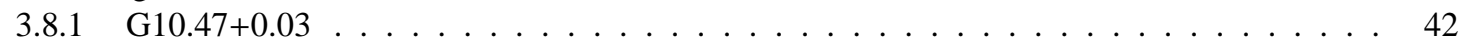

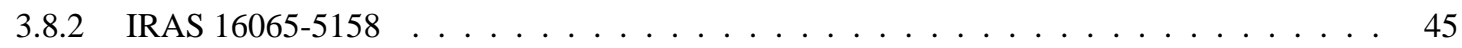

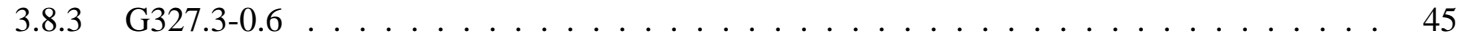

$3.8 .4 \mathrm{G} 34.26+0.15 \ldots \ldots \ldots \ldots \ldots \ldots \ldots \ldots$

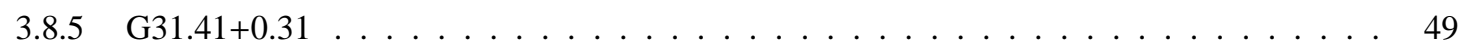

3.8 .6 IRAS $12326-6245 \ldots \ldots \ldots \ldots \ldots \ldots$

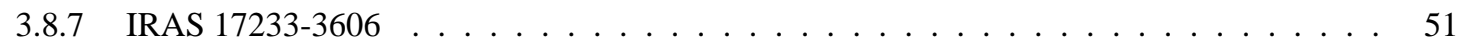

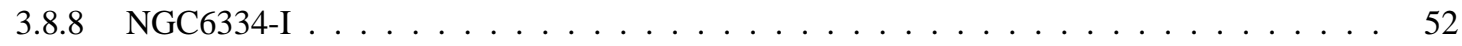

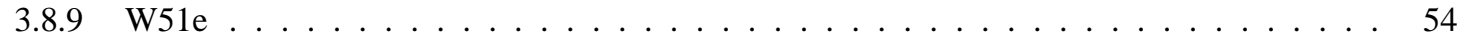

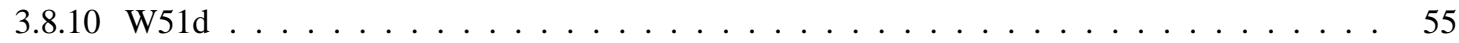

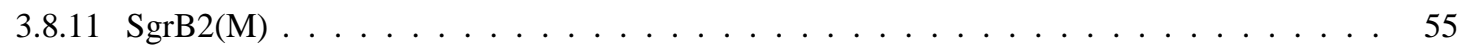

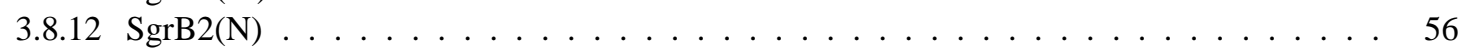

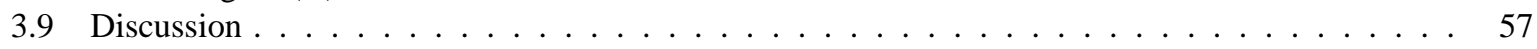

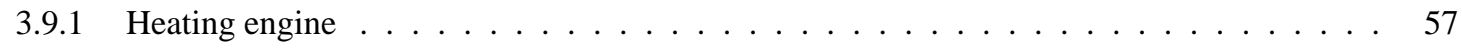

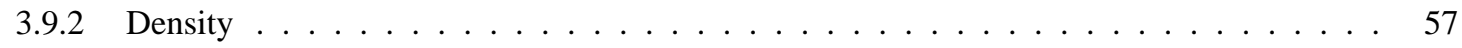

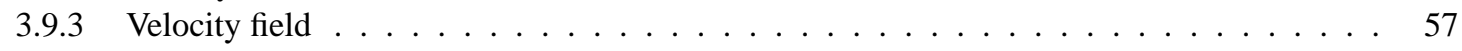

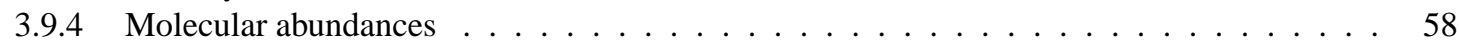

3.9 .5 Limitations of our modeling approach . . . . . . . . . . . . . . . . . . . . 58

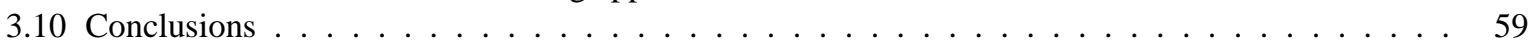

3.11 Additional Information . . . . . . . . . . . . . . . . . . . . . . . 60

4 Herschel paper: Reversal of infall in $\operatorname{SgrB2}(M)$ revealed by Herschel/HIFI observations of HCN lines at $\mathrm{THz}$ frequencies

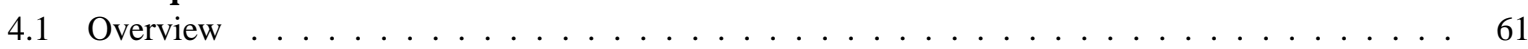

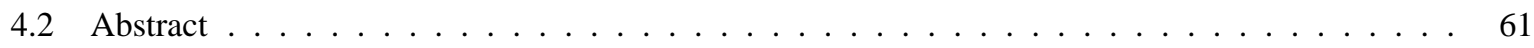

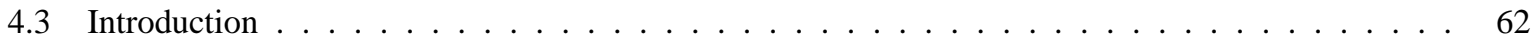

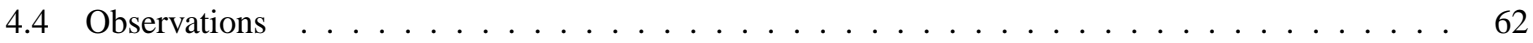

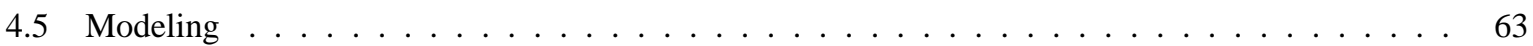

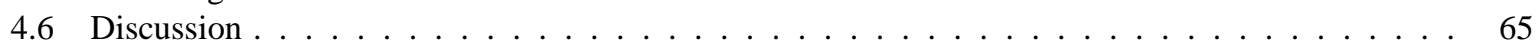

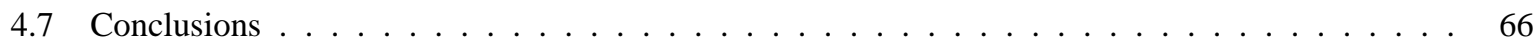

4.8 Additional Information . . . . . . . . . . . . . . . . . . . . . . 67

$4.8 .1 \mathrm{HCN}$ : More Frequencies . . . . . . . . . . . . . . . . . 67

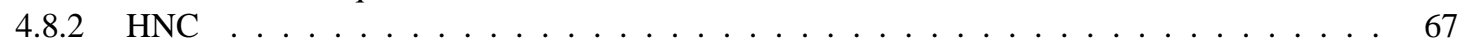

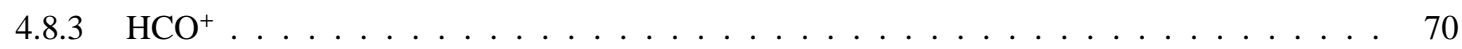

4.8.4 Variation of the abundances . . . . . . . . . . . . . . . . . . 70

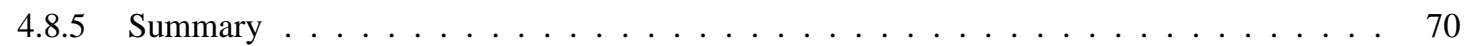


5 VLA paper: Hot HCN around young massive stars at 0.1" resolution 73

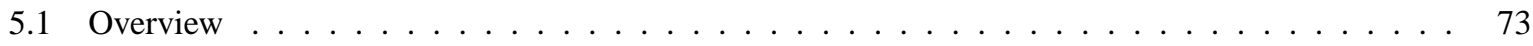

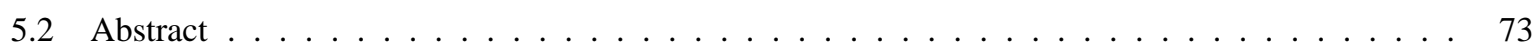

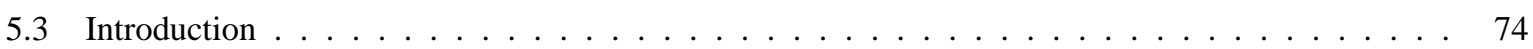

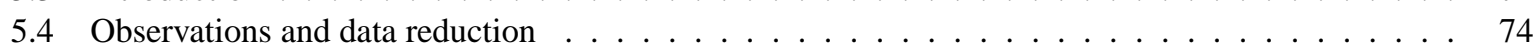

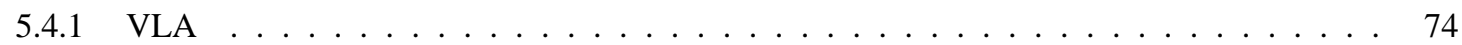

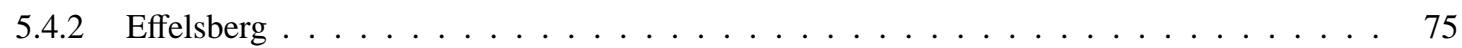

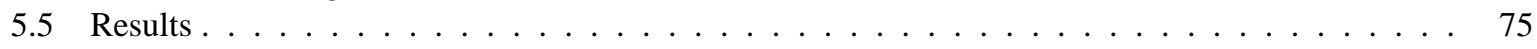

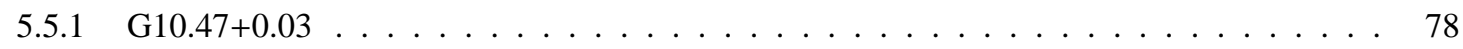

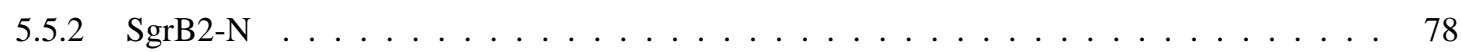

$5.5 .3 \quad$ SgrB2-M . . . . . . . . . . . . . . . . . . . . . . . 79

5.5 .4 Single-dish data . . . . . . . . . . . . . . . . . . . . . . . . . . . . . . . . . 79

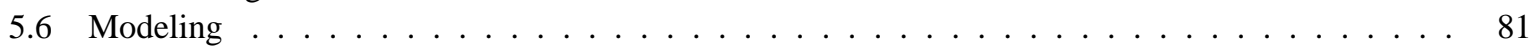

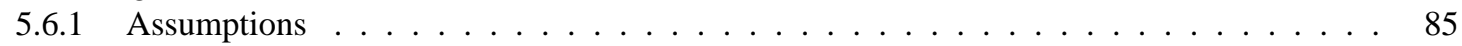

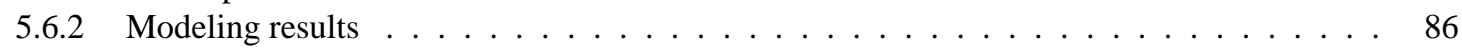

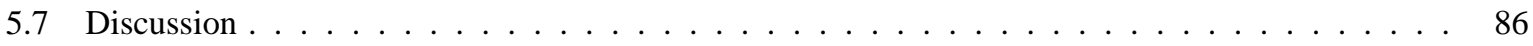

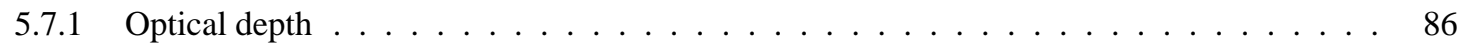

5.7 .2 Heating . . . . . . . . . . . . . . . . . . . . . . . . . . . . . . . 89

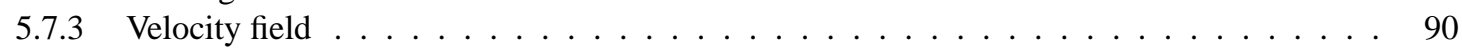

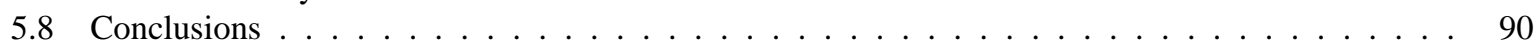

6 SMA paper: Structure of the Hot Molecular Core G10.47+0.03 93

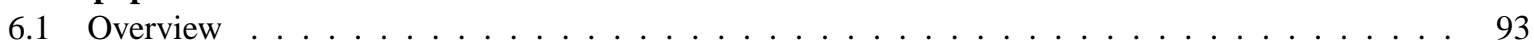

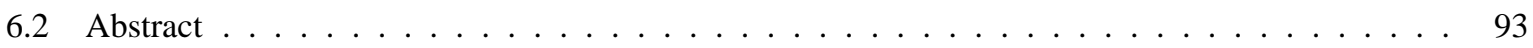

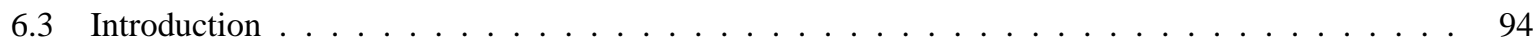

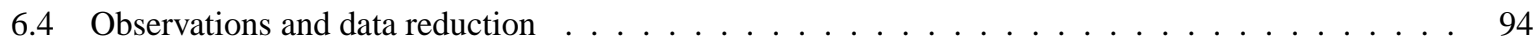

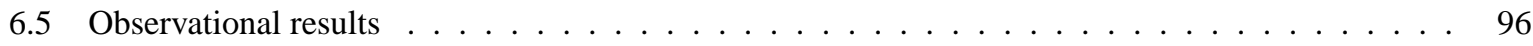

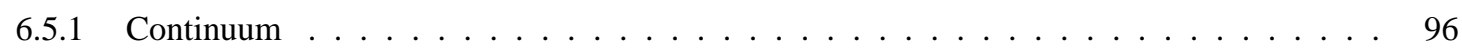

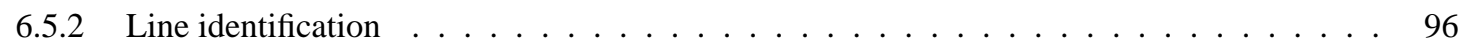

6.5 .3 Line maps . . . . . . . . . . . . . . . . . . . . . . . . . . . . . . . . 99

6.5 .4 Spectra . . . . . . . . . . . . . . . . . . . . . . . . . 99

6.5.5 Comparison to APEX data . . . . . . . . . . . . . . . . . . . . . 100

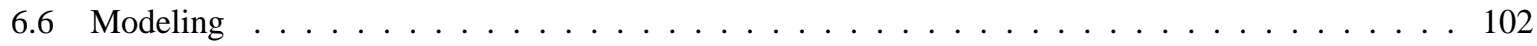

6.6 .1 The Models . . . . . . . . . . . . . . . . . . . . . . . . . . 102

6.6 .2 Continuum . . . . . . . . . . . . . . . . . . . . . . . . . . . . . 104

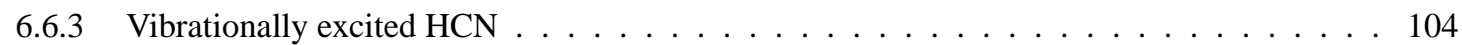

6.7 Discussion . . . . . . . . . . . . . . . . . . . . . . . . . . . . . . . 104

6.7 .1 Chemistry . . . . . . . . . . . . . . . . . . . . . . . . . . . 104

6.7 .2 Density Distribution . . . . . . . . . . . . . . . . . . . . . . 106

6.7 .3 Velocity Field . . . . . . . . . . . . . . . . . . . . . . . 110

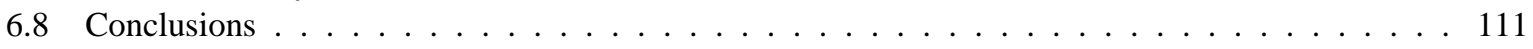

6.9 Appendix: Line maps . . . . . . . . . . . . . . . . . . . . . . . . . . . . . . 112

7 Additional SMA paper: Submillimeter Continuum Observations of Sagittarius B2 at Subarcsecond $\begin{array}{ll}\text { Spatial Resolution } & 117\end{array}$

$7.1 \quad$ Abstract . . . . . . . . . . . . . . . . . . . . . . . . . . . . . . . 117

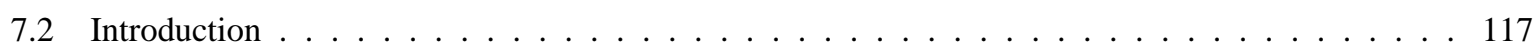

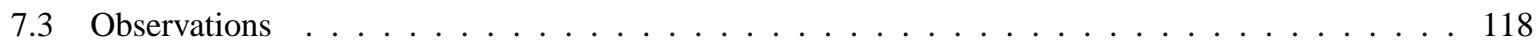

7.4 Results . . . . . . . . . . . . . . . . . . . . . . . . . . 118

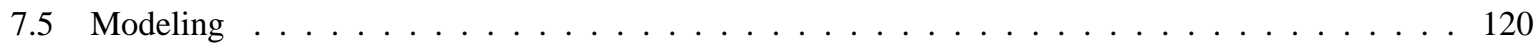

7.6 Discussion . . . . . . . . . . . . . . . . . . . . . . . . . 120

8 Discussion 125

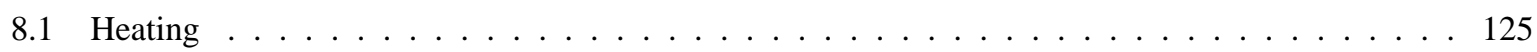

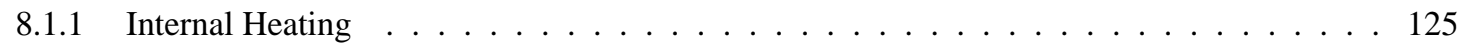

8.1 .2 Multiplicity of Heating Sources . . . . . . . . . . . . . . . . . . . . 125

8.2 Density Distribution . . . . . . . . . . . . . . . . . . . . . . . . . 126

8.2 .1 Radial Profile . . . . . . . . . . . . . . . . . . . . . . . . . . . . . . . . 126

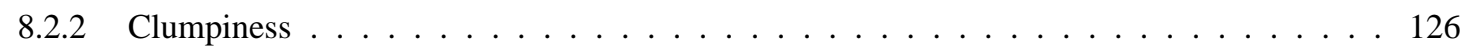


8.3 Velocity Field . . . . . . . . . . . . . . . . . . . . . . . . . . . 127

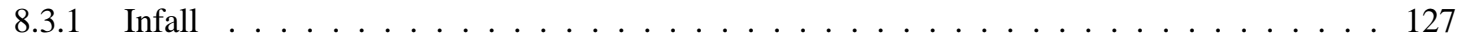

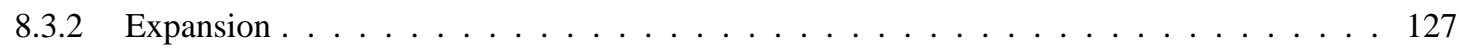

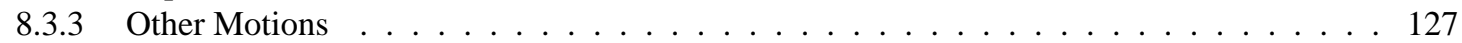

8.4 Molecular Abundances . . . . . . . . . . . . . . . . . . . . . . . . . . . 127

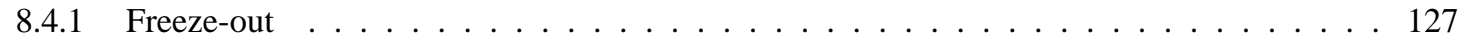

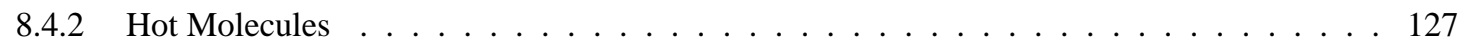

8.5 Implications for High-Mass Star Formation . . . . . . . . . . . . . . . . . . . . . . . 128

8.5.1 Large Mass of Hot Gas . . . . . . . . . . . . . . . . . . . . . . . . . 128

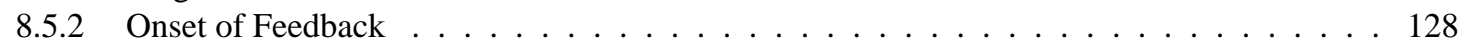

8.5.3 Distribution of Matter . . . . . . . . . . . . . . . . . . 128

9 Conclusions $r$\begin{tabular}{l}
129 \\
\hline
\end{tabular}

10 Outlook $\quad 131$

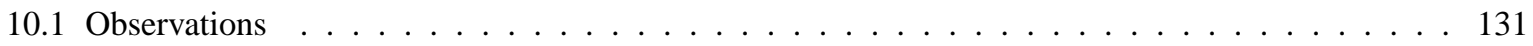

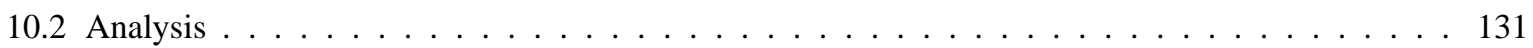




\section{Zusammenfassung}

Massereiche Sterne entstehen tief im Inneren von dichtem molekularem Gas, welches sie aufgrund ihres hohen Energieausstoßes aufheizen und ionisieren. Während einer frühen Phase ist die Ionisation auf kleine Bereiche beschränkt und die stellare Strahlung wird von Staub absorbiert. Die hohen Temperaturen führen zum Verdampfen der Eismäntel der Staubkörner, und viele hoch angeregte und komplexe Moleküle können in diesen heißen molekularen Kernen beobachtet werden. In späteren Stadien wird die ganze Molekülwolke ionisiert und auseinandergerissen, und ein Sternhaufen wird sichtbar.

Diese Doktorarbeit hat zum Ziel, die Verteilung von Dichte, Temperatur, molekularer Häufigkeit und Geschwindigkeitsfeld in heißen molekularen Kernen näher zu bestimmen. Diese Schlüsselinformationen für die Entstehung massereicher Sterne und für die Astrochemie werden durch aufwendige Strahlungstransport-Modellierung sowohl von Einzelteleskop- als auch von Interferometer-Beobachtungen gewonnen.

Zwölf Quellen wurden mit dem APEX-Teleskop in Submillimeter-Linien der Moleküle HCN, $\mathrm{HCO}^{+}$und $\mathrm{CO}$ beobachtet, wobei ein weiter Bereich von Anregungen und optischen Tiefen abgedeckt wurde. Dies wurde mitt dem Herschel-Teleskops ergänzt, welches HCN-Linien in SgrB2-M bis zu hohen (THz-) Frequenzen und Anregungen beobachtete. Die Linienformen und -intensitäten wurden mit dem sphärischen Strahlungstransportprogramm RATRAN unter den Annahmen einer radialen Potenzgesetz-Verteilung der Dichte und eines zentralen Heizens modelliert.

Vibrationsangeregtes HCN und ionisiertes Gas wurden mit dem VLA-Radiointerferometer bei einer hohen Auflösung von 0.1" (1000 AU) in G10.47+0.03, SgrB2-M und -N kartiert. Das SMA-Interferometer wurde eingesetzt, um Hunderte von Moleküllinien und Staubemission in G10.47+0.03 zu beobachten, wobei eine Frequenz von $690 \mathrm{GHz}$ und eine Auflösung von 0.3" erreicht wurden. Die Daten wurden mit dem dreidimensionalen Strahlungstransportprogramm RADMC-3D modelliert, welches die Staubtemperatur aus dem stellaren Aufheizen berechnet.

Modellieren mit einer Potenzgesetz-Dichtestruktur reproduziert die meisten Einzelteleskop-Linien, aber die hochaufgelösten Daten zeigen eine zentrale Abflachung und eine schnelle radiale Abnahme der Dichte und ähneln einem Plummer-Profil. Das Modellieren der Linienformen deutet auf kleinskalige Dichteschwankungen hin. Internes Heizen durch massereiche Sterne ist konsistent mit den Daten und macht sich durch vibrationsangeregtes HCN um kleine Regionen von ionisiertem Gas bemerkbar. Strahlungsdiffusion aufgrund der hohen Säulendichten führt zu Hunderten von Sonnenmassen an heißem (>300 K) Gas. Die HCN-Häufigkeit nimmt mit der Temperatur $\mathrm{zu}$ und erreicht in heißem Gas hohe Werte der Größenordnung $10^{-5}$ relativ zu $\mathrm{H}_{2}$. Großskaliger Einfall macht sich durch asymmetrische Linienformen bemerkbar und ist langsamer als der freie Fall, während zur selben Zeit zentrale Expansionsbewegungen durch blauverschobene Absorption und eine sich mit höherer Anregung ändernde Asymmetrie nachgewiesen sind.

Ich stelle fest, dass heiße molekulare Kerne bei anhaltendem Gravitationseinfall durch die beginnende Rückkopplung massereicher Sterne auf das Gas gekennzeichnet sind. Der erhöhte thermische, turbulente, windgetriebene und Strahlungsdruck im zentralen Bereich führt zu Expansionsbewegungen und zu einem zentralen Abflachen der Dichte. Hohe Temperaturen werden mittels Diffusion der Strahlung durch Staub erreicht. 


\section{Abstract}

High-mass stars form deeply embedded in dense molecular gas, which they heat up and ionize due to their high energy output. During an early phase, the ionization is confined to small regions, and the stellar radiation is absorbed by dust. The high temperatures lead to the evaporation of ice mantles around dust grains, and many highly excited and complex molecules can be observed in these Hot Molecular Cores. At later stages, the whole molecular cloud is ionized and disrupted, and a star cluster becomes visible.

This $\mathrm{PhD}$ thesis aims at constraining the distribution of density, temperature, molecular abundances, and velocity field in Hot Molecular Cores. This key information for high-mass star formation and for astrochemistry is obtained by sophisticated radiative transfer modeling of both single-dish and interferometric observations.

With the APEX telescope, 12 sources were observed in submillimeter lines of the $\mathrm{HCN}, \mathrm{HCO}^{+}$, and $\mathrm{CO}$ molecules, covering a wide range of excitations and optical depths. This was extended with the Herschel space telescope, which observed HCN lines in SgrB2-M up to high (THz) frequencies and excitations. The line shapes and intensities were modeled with the spherical radiative transfer code RATRAN, assuming a radial power-law density distribution and central heating.

With the VLA radio interferometer, vibrationally excited $\mathrm{HCN}$ and ionized gas was mapped at a high resolution of $0.1^{\prime \prime}$ (1000 AU) in G10.47+0.03, SgrB2-M and -N. The SMA interferometer was used to observe hundreds of molecular lines and dust emission in G10.47+0.03, reaching a frequency of $690 \mathrm{GHz}$ and a best resolution of $0.3^{\prime \prime}$. The data were modeled with the three-dimensional radiative transfer code RADMC-3D, which computes the dust temperature from stellar heating.

Modeling using a power-law density structure reproduces most single-dish lines, but the high-resolution data show a central flattening and a rapid radial decrease of the density, resembling a Plummer profile. Modeling of the line shapes indicates small-scale clumpiness. Internal heating by high-mass stars is consistent with the data and traced by vibrationally excited $\mathrm{HCN}$ around small regions of ionized gas. Diffusion of radiation due to the high column densities lead to hundreds of solar masses of hot $(>300 \mathrm{~K})$ gas. The HCN abundance increases with temperature, reaching high values on the order of $10^{-5}$ relative to $\mathrm{H}_{2}$ in the hot gas. Large-scale infall is traced by asymmetric line shapes and is slower than free-fall, while at the same time central expansion motions are detected by blue-shifted absorption and a change of the asymmetry with higher excitation.

I conclude that Hot Molecular Cores are characterized by the beginning feedback from high-mass stars, while gravitational infall is ongoing. The increased thermal, radiative, turbulent, and wind-driven pressure in the central region leads to expansion motions and to a central flattening of the density. High temperatures are reached through diffusion of radiation by dust. 


\section{Chapter 1}

\section{Introduction}

This PhD thesis was conducted at the Max-Planck-Institut für Radioastronomie in Bonn and the I. Physikalisches Institut at the University of Cologne. I worked on it from March 2008 to April 2011. The thesis advisor was Peter Schilke.

This introductory chapter gives background information and highlights the scientific context of the thesis, which is high-mass star formation. Starting from the conditions for star formation (Sect. 1.1), the peculiarities of the formation of high-mass stars are described in Sect. 1.2. Section 1.3 gives details about Hot Molecular Cores, an evolutionary stage of high-mass star formation prominent for molecular line emission. Problems in observations, analysis, and theory are discussed in Sect. 1.4. Finally, the aims of this thesis are defined in Sect. 1.5.

The next chapter (Chapter 2) describes the tools used in this work. As the measurement of electromagnetic radiation plays a key role for the whole science of astronomy, the first part (Sects. 2.1 to 2.4) is about radiative transfer, with an emphasis on molecular lines. Section 2.5 describes the observational techniques to measure radio and submillimeter wavelengths (single-dish and interferometry), and Sect. 2.6 introduces the modeling tools that are used to analyze the observational data. They basically simulate the radiative transfer and the observations of a model, to be compared to the real data.

Chapters 3 to 6 form the main part of the thesis. They rely on observations made with the APEX 12-m telescope (Chapter 3), the Herschel Space Observatory (Chapter 4), the VLA radio interferometer (Chapter 5), and the SMA submillimeter interferometer (Chapter 6). I present and discuss the observational data and the analysis with radiative transfer modeling in these chapters. They are based on papers that I have written. The papers of Chapters 3, 4, and 5 are refereed and published in the journal Astronomy E Astrophysics (Rolffs et al. 2011b, 2010, 2011a), the paper of Chapter 6 (Rolffs et al. 2011c) is submitted to the same journal. To Chapter 7 I contributed only the modeling part (Sect. 7.5), but reproduce the whole letter submitted to $A \mathcal{E} A$ (Qin et al. 2011) in order to embed Sect. 7.5 in its context.

In Chapter 8, I discuss the results from all observations and modeling, focusing on heating (Sect. 8.1), the density distribution (Sect. 8.2), the velocity field (Sect. 8.3), and the implications for astrochemistry (Sect. 8.4) and high-mass star formation (Sect. 8.5). Finally, I draw conclusions in Chapter 9 and give a short outlook in Chapter 10 .

\subsection{Pressure in the Interstellar Medium}

The Interstellar Medium (ISM) is kept in the plane of the Galaxy by the gravitational potential of the stars. In addition, the dense (molecular) clouds are held together by self-gravity. Turbulence plays an important role on all scales, rendering the ISM highly dynamic (e.g. Ballesteros-Paredes et al. 1999; Mac Low \& Klessen 2004). This is in contrast to the old picture of pressure equilibrium (Spitzer 1978), which is characterized by stable phases.

\subsubsection{Heating and Cooling}

The temperature of the ISM depends on (past and present) heating and the efficiency of cooling. Heating is basically the conversion of other forms of energy into thermal energy, which is genererally backed by the tendency to increase the entropy. Cooling is the conversion of thermal energy into photons, which are dispersed in space. It depends on the temperature, while heating is independent of it.

Above $10^{6} \mathrm{~K}$, the gas cools slowly by free-free radiation. At lower temperatures, internal levels of the gas particles have to be excited by collisions to decay radiatively, which depends on the availability of levels that can be excited. Above $10^{4} \mathrm{~K}$, electronic levels of atoms and ions are easily excited, and cooling is very efficient, but 
lower temperatures are not sufficient to excite these levels. Excitation of fine-structure lines, such as the $\mathrm{C}^{+}$line at $158 \mu \mathrm{m}$, can cool down to about $100 \mathrm{~K}$.

The formation of molecules starts the next step of cooling by providing further efficient mechanisms. As molecules have many low-lying rotational (and vibrational) levels, the gas can cool e.g. by rotational transitions of $\mathrm{CO}$ and $\mathrm{H}_{2} \mathrm{O}$. In addition, dust (about $1 \%$ of the mass) cools by continuum radiation, and influences the gas temperature through collisions with the molecules, especially at high density.

Main heating sources of the ISM are stellar radiation and supernovae. The first provides an approximately constant heating through absorption of the local interstellar radiation field. The latter is a rare explosive event, inducing not only radiation energy, but especially kinetic energy. Shocks and the decay of turbulence subsequently heat up the gas. In dense molecular clouds, which are shielded from the interstellar star light, absorption of the cooling radiation itself, the conversion of gravitational energy, and cosmic rays provide additional heating, maintaining a minimum temperature of about $10 \mathrm{~K}$, typical before the onset of star formation.

Considering the efficiency of cooling and heating by stellar radiation, three phases can be distinguished in a simplified picture of the ISM (McKee \& Ostriker 1977) - a hot ionized phase of $10^{6} \mathrm{~K}$ produced by supernovae, a warm phase of $10^{4} \mathrm{~K}$ (which can have different degrees of ionization), and a cold neutral phase of about $100 \mathrm{~K}$. The thermal pressure, which is the product of particle density and temperature, would be $n T \approx 3000 \mathrm{~cm}^{-3} \mathrm{~K}$ outside of molecular clouds (where gravity is crucial), leading e.g. to a few dozen atoms per $\mathrm{cm}^{3}$ for the cold phase. The volume of the Milky Way is thought to be about equally distributed between the hot and the warm phase, the gas mass $\left(\sim 10^{10} \mathrm{M}_{\odot}{ }^{1}\right)$ between the warm and the cold phase. $\sim 10 \%$ of the gas mass is molecular.

The modern picture emphasizes much more the turbulent and dynamic nature of the ISM, which is out of equilibrium. The lifecycle of interstellar matter involves gradual cooling and contracting until star formation sets in, and subsequent heating and disruption of the remaining gas. Through star formation, the ISM loses mass, which could be partly replenished from the intergalactic medium.

\subsubsection{Collapse}

Pressure gradients drive the acceleration of matter. Different types of pressure can be distinguished, e.g. thermal, gravitational, turbulent, rotational, or magnetic pressure. Gravity is the driving force for collapse, while most pressure types provide support against collapse. Turbulence provides support on global scales, but can promote local collapse at the same time (Klessen et al. 2004).

At high densities, self-gravity becomes important, exerting a contraction force. Thermal and turbulent pressure represent microscopic and macroscopic motions of the particles, respectively. In addition, magnetic pressure tends to stabilize a cloud against collapse, since charged particles are coupled to the magnetic field, which resists compression. Neutral and charged particles can slowly decouple by ambipolar diffusion.

For collapse to occur, gravity has to ultimately win over all other forces. A simple approximation to estimate the critical mass, at which the cloud is stable, is to evaluate the energy balance in virial equilibrium

$$
2 \mathcal{T}+2 \mathcal{U}+\mathcal{M}+\mathcal{W}=0
$$

For a cloud of mass $M$ and radius $R$, the internal energy (kinetic energy of macroscopic motion) is $\mathcal{T} \propto M v^{2}$ with $v$ the typical velocity, the thermal energy is $\mathcal{U} \propto M T$, the magnetic energy is $\mathcal{M} \propto B^{2} R^{3}$ with the field strength $B$, and the gravitational (or potential) energy is $\mathcal{W} \propto-\frac{M^{2}}{R}$. For collapse, the gravitational energy must be larger than the rest. However, this is very simplified and ignores surface terms, also it is not always clear if the condition of equilibrium is fulfilled (Ballesteros-Paredes 2006).

A simple case is to consider only gravitational and thermal energy: Then $\mathcal{U} \propto \mathcal{W}$, or $T \propto \frac{M}{R}$, therefore $M \propto \rho R^{3} \propto \rho \frac{M^{3}}{T^{3}}$. Division by $M$ and multiplication by $\frac{T^{3}}{\rho}$ leads to the Jeans mass $M_{\mathrm{J}} \propto \sqrt{\frac{T^{3}}{\rho}}$. For a temperature of $10 \mathrm{~K}$ and a density of $10^{4} \mathrm{H}_{2}$ molecules per $\mathrm{cm}^{3}$, it is about $1 \mathrm{M}_{\odot}$, while for diffuse neutral gas it can be some $10^{4} \mathrm{M}_{\odot}$.

In the complete absence of supporting forces, a cloud would collapse over the free-fall time $t_{\mathrm{ff}}$. The core radius $R$ is roughly the acceleration times the square of this time, $R \approx \frac{G M}{R^{2}} t_{\mathrm{ff}}^{2}$, so $t_{\mathrm{ff}} \approx(G \rho)^{-\frac{1}{2}}$. For a neutral cloud, the free-fall time is around $10^{7}$ years, for a dense core only some $10^{4}$ years.

With increasing density, self-gravity becomes stronger. Smaller masses can collapse and the timescales are shorter. Collapse thus proceeds first in the dense, inner regions, with outer regions following (inside-out collapse, Shu 1977). This might be different if the cloud is initially homogeneous, in which case the collapse propagates inward (Larson-Penston collapse, Larson 1969a; Penston 1969). A comparison between these solutions can be found in Zhou (1992).

\footnotetext{
${ }^{1}$ Astronomical constants in this thesis are solar mass $\mathrm{M}_{\odot}=1.98892 \times 10^{30} \mathrm{~kg}$, solar luminosity $\mathrm{L}_{\odot}=3.839 \times 10^{26} \mathrm{~W}$, the Astronomical Unit AU $=1.49598 \times 10^{11} \mathrm{~m}$, and the parsec $\mathrm{pc}=3.08568025 \times 10^{16} \mathrm{~m}$. Physical constants are Planck's constant $h=6.626 \times 10^{-34} \mathrm{Js}$, the speed of light $c=2.99792458 \times 10^{8} \mathrm{~m} / \mathrm{s}$, Boltzmann's constant $k=1.38 \times 10^{-23} \mathrm{~J} / \mathrm{K}$, and the gravitational constant $G=6.6739 \times 10^{-11} \mathrm{~m}^{3} / \mathrm{kg} / \mathrm{s}^{2}$.
} 
The supporting force is here represented by thermal pressure. However, in molecular clouds turbulence and magnetic fields dominate, increasing the stable mass. The far greater contribution of turbulence than of thermal pressure can be seen in the line widths, which are much larger than the thermal line width. The line width to the power of three is contained in the Jeans mass.

Nevertheless, since higher densities increase the self-gravity, the stable mass decreases and consecutively lower masses can contract. This is triggered by turbulent contractions. This gravoturbulent fragmentation leads to strong density fluctuations (cores inside clumps) in Giant Molecular Clouds, which typically measure $50 \mathrm{pc}$ and have masses of $10^{5} \mathrm{M}_{\odot}$. Molecular clouds are probably in a non-equilibrium state of overall gravitational collapse with many local centers of collapse (Ballesteros-Paredes et al. 2011).

Collapse first proceeds in an isothermal way, as the energy released by contraction, converted to thermal energy, can be radiated away easily. This is essential for the fragmentation. However, as the density increases, it becomes harder to get rid of the additional thermal energy, because cooling lines become optically thick, leading to trapping of line photons, and later also the dust emission is increasingly absorbed. The dust optical depth becomes even larger with higher temperature, as the emitted radiation is shifted to shorter (infrared) wavelengths.

When the isothermal approximation breaks down due to the large dust optical depth, the collapsing core can cool only at its surface, where accretion is ongoing, and the temperature rises. A first hydrostatic core develops, where thermal pressure balances gravity (Masunaga et al. 1998). This is the seed of a star.

\subsubsection{Expansion}

As soon as stars have formed, they provide additional pressure to the ISM. They convert gravitational and nuclear binding energy into radiation, produce winds and, during the accretion, bipolar outflows.

These feedback mechanisms strongly enhance the turbulence and also the thermal pressure by heating the gas. Especially the high-mass stars also provide radiation pressure and ionize the surrounding gas by their strong UV radiation. The ionized gas has high pressure due to its temperature of $10^{4} \mathrm{~K}$. The combined pressure from the fast stellar winds that high-mass stars drive, their radiation pressure, and the thermal pressure of the ionized gas finally removes the gas from a newly formed star cluster. Having lost a substantial part of their mass, the less massive clusters disssolve.

The feedback on the star formation process itself is mostly negative, since the additional pressure works against gravity. However, it can also be positive and trigger star formation elsewhere by compressing the gas locally (Deharveng \& Zavagno 2008; Zavagno et al. 2007, 2010).

At later stages of stellar evolution, massive winds can lead to an expansion of stellar envelopes, and high-mass stars explode after a few million years as supernovae, sweeping up and heating the ISM on large scales. These processes enrich the ISM with metals and close the cycle of interstellar matter.

\subsection{High-Mass Star Formation}

A detailed overview of star formation, including also the topic of the last section (Sect. 1.1), can be found in the book by Stahler \& Palla (2005). A review on high-mass star formation is Zinnecker \& Yorke (2007).

\subsubsection{Impact}

The formation of high-mass stars, through its feedback on the ISM, regulates the star formation process. Together with the high energy output, it thus shapes and dominates the evolution and appearance of galaxies. This is particularly true for starburst galaxies and earlier cosmic epochs, which were characterized by high star formation rates triggered by galaxy mergers. The first stars in the universe must have had very high masses, as the gas temperatures were high due to the lack of cooling mechanisms. Mainly through the production and disposal of heavy elements, high-mass stars provide the conditions for the formation of dust, planets, and life.

High-mass stars have large enough masses that they collapse to a neutron star or a black hole when the raw material of nuclear fusion is exhausted and its support against gravity vanishes. The released potential energy causes a supernova explosion, outshining the whole galaxy and creating giant bubbles in the ISM. This criterion means that they have masses above $\sim 8 \mathrm{M}_{\odot}$. According to the stellar initial mass function (IMF, Kroupa 2002), only $0.4 \%$ of newly formed stars have high enough masses. However, as the luminosity on the Main Sequence (MS, which relates the luminosity to the surface temperature of hydrogen-burning stars) is proportional to the mass to the power of about 3.5, they dominate the luminosity, but use up the hydrogen fuel very quickly (lifetime on the MS proportional to $M / L$, about $10^{10}$ years for the Sun). So high-mass stars are short-lived, and most of their material is recycled to the ISM. In contrast, the matter is essentially locked in the $94 \%$ of newly formed stars that have masses lower than $1 \mathrm{M}_{\odot}$. 


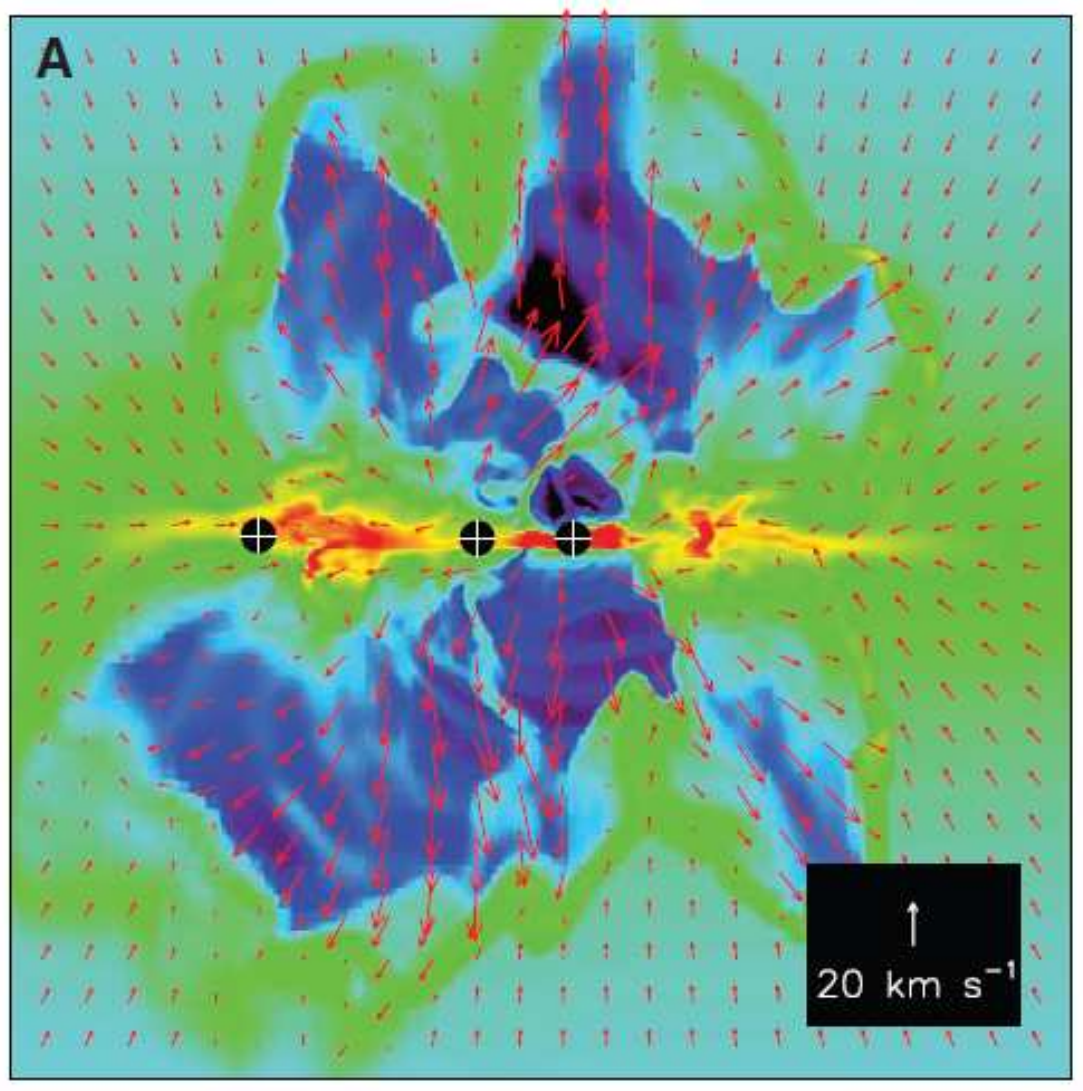

Figure 1.1: High-mass stars forming via disk accretion (simulation by Krumholz et al. 2009). The color scale represents logarithmic density, the arrows denote the gas velocity.

Already before the supernova explosions, high-mass stars have a far greater impact on their natal molecular cloud than the low-mass stars. They have high luminosities and hot surface temperatures, therefore an ionizing radiation field, producing HII regions and photon dominated regions (PDR), where the molecules are dissociated. With their fast winds, they drive out the cluster gas.

\subsubsection{Accretion}

A fundamental difference between low-mass and high-mass star formation is that for low-mass stars hydrogen burning starts only after accretion and contraction have finished, while for high-mass stars it starts while still growing in mass. The virial equilbrium (Equation 1.1), which applies to a hydrostatic core, relates gravitational and thermal energy; hence contraction and higher mass mean higher temperatures, which eventually ignite hydrogen. For high-mass star formation, the Kelvin-Helmholtz time scale $R / \dot{R}=\frac{G M^{2}}{R L}$, over which the luminosity could be maintained by contraction, is less than the collapse time scale $M / \dot{M}$. The accretion rate $\dot{M}$ (infalling mass per time) produces a luminosity of $\frac{G M}{R} \dot{M}$. It depends on stellar mass and environment, and a high-mass star reaches values over $10^{-3} \mathrm{M}_{\odot} \mathrm{yr}^{-1}$ (Beuther et al. 2002; Keto \& Wood 2006).

The extreme luminosities of high-mass stars lead to high radiation pressure, which reverses any spherical accretion flow. Therefore, they can only form by an inhomogeneous non-spherical infall or by disk accretion. This way, the accreting material is shielded from the radiation, which can escape through expanding low-density bubbles (e.g. Fig. 1.1, Krumholz \& Matzner 2009).

Disk accretion is accompanied by bipolar outflows. In low-mass star formation, magneto-centrifugal outflows, powered by the accretion, are a common phenomenon. High-mass stars produce strong radiation-driven winds, which can entrail molecular gas in an outflow.

Outflows from young high-mass stars are much more massive (Beuther et al. 2002) and probably less collimated (Shepherd 2005) than from low-mass stars. Direct detection of disks is more difficult. Large massive but instable rotating structures, so-called toroids, have been observed (Beltrán et al. 2005; Cesaroni et al. 2007).

The ionization is first quenched by accretion, i.e. the accretion rate is larger than the ionization rate and so no HII region can be observed (Walmsley 1995). Accretion, with its large infalling momentum, can well proceed into HII regions as an ionized accretion flow (Keto 2003). Finally, the accretion disk is probably photoevaporated, 
which puts an end to accretion (Hollenbach et al. 1994), and the HII region undergoes the classical pressure-driven expansion (Spitzer 1978).

That accretion must occur through a disk is also set by the conservation of angular momentum. Forming stars have to get rid of angular momentum, which would prevent contraction otherwise. Therefore, matter has to flow in while angular momentum is transported outwards by friction in the disk or by outflows. Also the formation of binary or multiple stellar systems and of planets serve this purpose.

In addition to angular momentum, other factors limit and regulate star formation, whose relative importance is not clear though. Important contributions come from turbulence (Mac Low \& Klessen 2004), likely replenished by outflows (Li \& Nakamura 2006; Wang et al. 2010), and from magnetic fields (e.g. Hennebelle \& Fromang 2008; Peters et al. 2011). Also thermal pressure from heating, winds, and radiation pressure play a role.

While it has become clear that high-mass stars can form by accretion, although with substantial differences to low-mass stars, it is currently under debate how high-mass stars grow by accretion, with two rivaling theories. Monolithic collapse of turbulent-supported cores (McKee \& Tan 2003) assumes that a high-mass star has its own well-defined mass reservoir and accretion disk. In contrast, competitve accretion in a cluster environment (Bonnell et al. 2001, 2004) focuses on the global mass reservoir, accreted by the combined gravitational potential of the stars, which compete for this accretion flow by their location and their mass.

There is observational support for both scenarios, e.g. the outflows and the core mass function for monolithic collapse and the large-scale infall and the clustering of high-mass stars for competitive accretion. The truth lies probably in between, with aspects of both theories being real. In addition, stars may collide and merge in cluster centers, focused by their gravitational attraction. This coalescence scenario (Bonnell et al. 1998; Bally \& Zinnecker 2005) of merging low-mass stars applies however only in exceptional cases.

To conclude, the high and hard luminosity that high-mass stars produce very early in their formation is an obstacle to accretion, but not an unsurmountable one. The exact way of accretion is not yet known, however. The radiation is likely to limit the stellar mass, e.g. by photoevaporation of the accretion disk.

\subsubsection{An Evolutionary Sequence}

In low-mass star formation, a well-established evolutionary scheme is based on the Spectral Energy Distribution (SED) of a protostar, which reflects the relative importance of envelope/disk and the star itself. As the star is hotter, the SED is shifted to shorter wavelengths with time. From a prestellar core, a class 0 protostar emerges, which emits only in the submm regime (Andre et al. 1993). The star, which is completely obscured by the envelope, drives an outflow. It first appears in the SED as an infrared protostar, with the submm contribution from envelope and disk still dominating (class I). In class II, there is no more outflow and only weak accretion from a circumstellar disk, which gradually disappears (due to planet formation). Class III (T Tauri star) is a contracting pre-MS star with only remnants of the disk present.

While low-mass protostars can even be distinguished on a plot of luminosity vs. effective (surface) temperature as colder stars gaining their luminosity from contraction, there is no high-mass protostar in the sense of a pre-MS star (before the onset of hydrogen burning), but high-mass stars instead evolve up the MS by growing in mass. They cannot be observed optically there, however, as they are highly obscured by surrounding dust. Moreover, they evolve very quickly and do not form in isolation, but in a clustered mode. This makes it much more difficult to clearly define an evolutionary sequence for high-mass star formation.

Theoretically, Zinnecker \& Yorke (2007) distinguish between four phases: compression to dense cores or filaments by gravoturbulent fragmentation, collapse of parts of these cores into small protostars, accretion onto these protostars, and disruption of the whole molecular cloud by feedback from the high-mass stars.

Observationally, the most likely sequence of events has distilled out as the following. The highest column densities are sites of future high-mass star formation (Krumholz \& McKee 2008), and absorb the infrared radiation field. Visible as dark patches in the infrared background, they are called infrared dark clouds (IRDC, Menten et al. 2005; Rathborne et al. 2006). When high-mass stars have formed there, they first heat up the gas, giving rise to a Hot Molecular Core (HMC, Sect. 1.3). Next, they ionize the gas, and HII regions growing in size can be observed by their free-free radio emission (Hoare et al. 2007). Hypercompact HII regions have high densities $\left(>10^{6}\right.$ electrons per $\mathrm{cm}^{3}$ ), a rising spectral index, they are very small $(\sim 0.01 \mathrm{pc})$, and have broad radio recombination lines (Kurtz 2005). They probably evolve over ultracompact and compact to large, classical HiI regions. When they have expanded and disrupted the cloud, a star cluster or an OB association can be seen in visible light.

\subsection{Hot Molecular Cores}

As soon as stars have formed inside dense and cold cores, they heat up their surrounding gas. Ice mantles around dust grains evaporate, releasing complex molecules into the gas phase. Molecules are highly excited due to the 


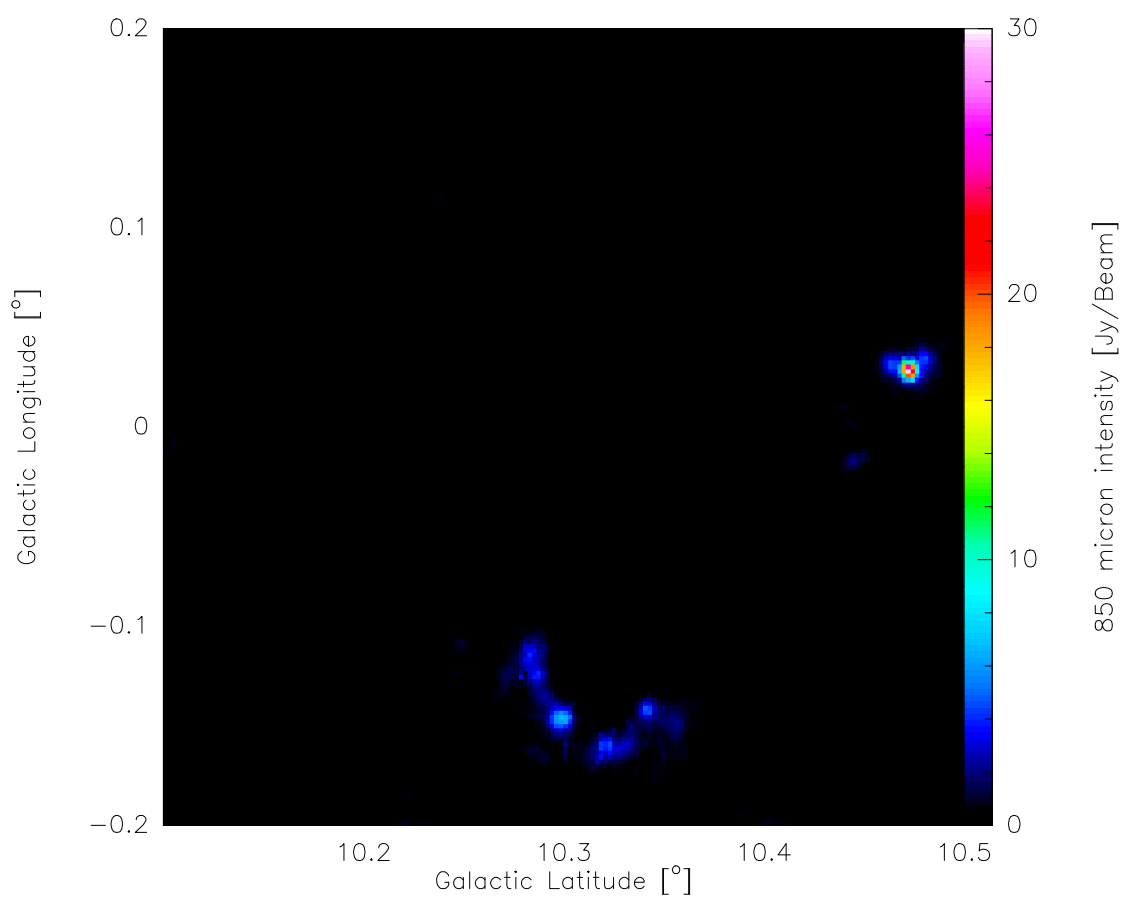

Figure 1.2: ATLASGAL map of a region of $0.4^{\circ}$ diameter ( 80 beam sizes). The compact source on the right side is the Hot Molecular Core G10.47+0.03, the color scale is Jy/Beam.

large temperatures of over $100 \mathrm{~K}$, and the large column densities together with the high temperatures lead to extraordinary emission of molecular lines. This stage is called a Hot Molecular Core.

It is characterized by the beginning of stellar feedback. Most obvious is the heating of dust and gas by the newly formed stars. The higher temperatures have also chemical implications, from the evaporation of grain mantles to gas-phase reactions that change the composition of the molecular gas.

Reviews of hot cores can be found e.g. in Kurtz et al. (2000), Churchwell (2002), van der Tak (2004), Cesaroni (2005), and Beuther (2007).

\subsubsection{Discovery}

Due to the high dust column densities, these sources are obscured in the mid-infrared and at shorter wavelenghts, so they look simply dark with classical optical telescopes. The frequency-dependence of dust absorption originates in the size distribution of dust grains, which are more abundant the smaller they are, and the properties of their coupling to electromagnetic radiation, which is stronger with higher ratio of grain size to wavelength.

With the advent of spectrally resolved radio astronomy and, in conjunction, molecular astrophysics, the existence of HMCs could be revealed. The long wavelength is needed to see through the dust, and the molecular lines to estimate the temperature by relative intensities of lines from different excitation states.

An important molecule was, and is, ammonia $\left(\mathrm{NH}_{3}\right)$. The inversion transitions of its metastable states lie relatively close in a well-observable frequency range $(1.3 \mathrm{~cm})$, and the hyperfine structure allows a derivation of optical depth (e.g. Morris et al. 1980; Cesaroni et al. 1994). But also $\mathrm{CH}_{3} \mathrm{CN}$ was used early to derive a temperature of $275 \mathrm{~K}$ in Orion (Loren \& Mundy 1984). A later example is the use of the vibrational states of $\mathrm{HC}_{3} \mathrm{~N}$ (Wyrowski et al. 1999).

The SED that hot cores emit peaks at around $100 \mu \mathrm{m}$, in the far infrared. This wavelength regime is completely absorbed by the Earth's atmosphere and not observable from the ground. In 1983, the space telescope IRAS (Infrared Astronomical Satellite) surveyed the whole sky at 12, 25, 60, and $100 \mu \mathrm{m}$, albeit with low angular resolution. HMC candidates can be selected by color criteria from the point source catalog, and investigated by further continuum and line observations (Sridharan et al. 2002; Araya et al. 2005; Dedes et al. 2011). SED (and line) studies of HMCs are about to improve significantly with data from the Herschel space telescope.

For high column densities $\left(\sim 10^{25} \mathrm{H}_{2} \mathrm{~cm}^{-3}\right)$, dust changes between optically thin and thick at around $1 \mathrm{~mm}$. In this regime, most of the dust emission can be actually seen, and HMCs appear as the strongest sources in the sky due to their large masses and high temperatures. For instance, in the $850 \mu \mathrm{m}$ survey of the inner Galactic plane ATLASGAL (APEX Telescope Large Area Survey of the Galaxy, Schuller et al. 2009), HMCs stick out prominently as compact and strong sources (Fig. 1.2). 


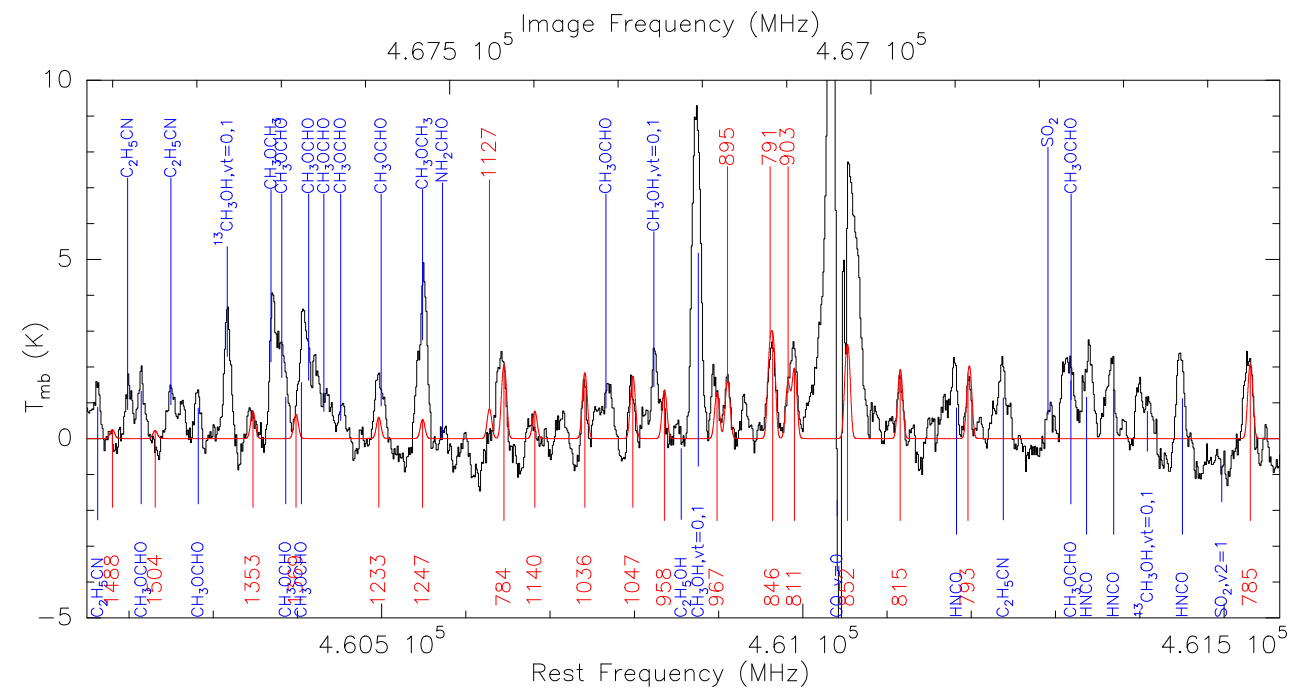

Figure 1.3: APEX observations of the Hot Molecular Core G327.3-0.6 (double-sideband mode), overlaid with a model from myXCLASS for $\mathrm{CH}_{3} \mathrm{CN}, v_{8}=1$ (red, the size is $1.5^{\prime \prime}$, the temperature $300 \mathrm{~K}$, the line width $4 \mathrm{~km} / \mathrm{s}$ and the $\mathrm{CH}_{3} \mathrm{CN}$ column density is $10^{17} \mathrm{~cm}^{-2}$ ). The energy of the lower level is given in $\mathrm{K}$.

Embedded high-mass stars, whose entire radiation is absorbed by dust and converted to the infrared, can already have developed an HII region, which emits free-free radiation in the radio regime. As their dust emission shows up in the IRAS maps, Wood \& Churchwell (1989) searched for ultracompact HII regions in color-selected IRAS sources. Due to the clustering of high-mass stars, one can expect to find HMCs close to them. Also, HMCs can be heated by the star(s) in the UCHII regions, in which case it is considered as external heating, in contrast to heating by internal sources which have not yet developed a UCHII region.

Finally, HMCs are often associated with water masers (at $22 \mathrm{GHz}$ ), which require several hundred $\mathrm{K}$ and $>10^{8}$ $\mathrm{cm}^{-3}$ to be excited. Also $\mathrm{OH}$ and $\mathrm{CH}_{3} \mathrm{OH}$ masers occur. Masers are very strong, non-thermal lines which arise from population inversion. They are very compact and easily detectable.

Ouflows also often appear in HMCs, but to definitely detect a hot core, the spectrum has to show many lines from complex organics and highly excited states (e.g. Fig. 1.3).

\subsubsection{Chemistry}

Chemistry in the ISM differs signifcantly from chemistry on Earth due to the low number densities, which prevent three-body encounters (e.g. Tielens 2005). Therefore, reactions with a single product cannot happen due to energy and momentum conservation. Also the low temperatures typical in molecular clouds $(\sim 10 \mathrm{~K})$ prevent endothermic reactions or reactions with an activation barrier. Instead, charge-transfer reactions between neutral and ion dominate the gas-phase chemistry. A typical sequence of events is the ionization of $\mathrm{H}_{2}$ by cosmic rays, the formation of $\mathrm{H}_{2}^{+}, \mathrm{H}_{3}^{+}$, and $\mathrm{HCO}^{+}$by collisions with $\mathrm{H}_{2}$ and $\mathrm{CO}$. Subsequent reaction pathways can lead to larger molecules, which finally recombine with an electron and lose (at least) a hydrogen atom. The products of such gas-phase reactions tend to be rather poor in hydrogen (e.g. carbon-chain molecules).

The majority of molecules in the ISM were found in HMCs, including complex molecules (Herbst \& van Dishoeck 2009). With the high temperatures and large densities, gas-phase chemistry in HMCs is somewhat richer than in other molecular environments, in particular activation barriers can be overcome and endothermic reactions can occur. Probably even more important is grain-surface chemistry, as the released binding energy can be given to the dust grain and more reactions are possible. Atoms and molecules stick to the grains when they collide, and ice mantles form at low temperatures. Hydrogen atoms can move on the surface, and at higher temperatures also heavier atoms/molecules can move. This way, the hydrogen atoms scan the grain until they find a reaction partner and form the most abundant molecule $\mathrm{H}_{2}$ (Duley \& Williams 1993) as well as saturated molecules such as $\mathrm{H}_{2} \mathrm{O}$ (water), $\mathrm{NH}_{3}$ (ammonia) and $\mathrm{CH}_{3} \mathrm{OH}$ (methanol).

The astrochemistry of HMCs thus develops from freezing out of molecules to form ice mantles around dust grains in a cold phase, the warm-up with increased mobility and reacitivity and eventual evaporation of the ices when the newly formed stars provide heating, and gas-phase chemistry in a hot phase. Garrod et al. (2008) have simulated the reactions occuring in gradual warm-up of the grain mantles and the gas-phase, leading to varying abundances of the molecules. In principle, such simulations can provide age estimates of HMCs, as chemical timescales are long enough in interstellar conditions and the gas does not reach a chemical equilibrium. However, 
this requires detailed knowledge of the history of collapse and warm-up for different parts of the cores, as well as reliable reaction networks also at high temperatures.

An indication of age could be e.g. the abundances of $\mathrm{SO}$ and $\mathrm{SO}_{2}$, as they seem to differ substantially between sources. Sometimes, N- and O-bearing molecules are found at different locations, which could be an age or temperature effect (Rodgers \& Charnley 2001). Very complex reaction networks and history determine the abundances of the different molecules, starting from the elemental abundances, which in the solar neighborhood are relative to hydrogen $0.07(\mathrm{He}), 6.8 \times 10^{-4}(\mathrm{O}), 3.7 \times 10^{-4}(\mathrm{C}), 1.2 \times 10^{-4}(\mathrm{~N})$, and $1.6 \times 10^{-5}(\mathrm{~S})($ Cameron 1973$)$.

\subsubsection{Physical Structure}

The physical structure of HMCs is not well known, and differs from source to source. Most sources have masses on the order of $10^{3} \mathrm{M}_{\odot}$, and luminosities on the order of $10^{5} \mathrm{~L}_{\odot}$, which is reprocessed by dust and mostly emitted in the far infrared.

The density reaches values well above $10^{7} \mathrm{H}_{2} \mathrm{~cm}^{-3}$ in the central region and falls off with radius. This condensation of matter is expected from collapse. Some HMCs show fragmentation in the sense of multiple centers. It is not clear how to define the size for a continuous density distribution; the area where most of the dust and line emission is concentrated (if observed with an interferometer) is typically on the order of $0.1 \mathrm{pc}$.

The temperature is higher in the inner part than further outside. This temperature gradient points to internal heating by the formation of embedded stars or protostars. Average temperatures, deduced from line ratios, are $100-300 \mathrm{~K}$, but probably all temperatures up to the dust sublimation temperature of around $1500 \mathrm{~K}$ are present on small scales.

Apart from turbulence, which is imprinted in the line widths, there are many indications for systematic motions such as outflows, rotation, and collapse. Velocity gradients seen at high spatial resolution can be interpreted as rotating massive disks or toroids (Beltrán et al. 2005, 2011; Zapata et al. 2010). Outflows can be observed on larger scales and in high-velocity line wings. Simultaneous infall often shows up in asymmetric line profiles or in red-shifted absorption (e.g. Qiu et al. 2011; Furuya et al. 2011).

\subsubsection{Sources}

A few dozen sources are currently known in the Galaxy, and they display a high individuality. The sources investigated in this thesis are briefly described in Chapter 3, as they were all observed with the APEX telescope (Table 3.1). These are G10.47+0.03 (Sect. 3.8.1), IRAS 16065-5158 (Sect. 3.8.2), G327.3-0.6 (Sect. 3.8.3), G34.26+0.15 (Sect. 3.8.4), G31.41+0.31 (Sect. 3.8.5), IRAS 12326-6245 (Sect. 3.8.6), IRAS 17233-3606 (Sect. 3.8.7), NGC6334-I (Sect. 3.8.8), W51e (Sect. 3.8.9), W51d (Sect. 3.8.10), SgrB2-M (Sect. 3.8.11), and SgrB2-N (Sect. 3.8.12).

SgrB2-M was also observed with Herschel (Chapter 4), and G10.47+0.03, SgrB2-M and -N with the VLA (Chapter 5) and the SMA (G10.47+0.03 in Chapter 6, continuum of SgrB2 in Chapter 7). They are all extremely massive HMCs associated with $\mathrm{HCH}$ II regions.

Among the other HMCs, Orion-KL is the most prominent source with a distance of only $414 \mathrm{pc}$ (Menten et al. 2007). It could be an unusual hot core though, heated by a nearby explosive event rather than internal high-mass stars (Zapata et al. 2009b, 2011).

\subsection{Problems}

The understanding of high-mass star formation and of astrochemistry has to face several problems in theory, observations, and analysis. Some of them are described in the following.

\subsubsection{Theory}

Although much progress has been made in the last years, it is still not well understood how high-mass stars form. Examples of problems include the relative contribution of different forms of pressure to the support against gravity, the exact ways of collapse and accretion, and the effects of feedback.

High-mass stars form only in clusters (Lada \& Lada 2003) and usually in multiple systems, while low-mass star formation can also occur in relative isolation. Furthermore, they are concentrated in cluster centers (mass segregation), and it is under debate if they formed there or have migrated there due to dynamical interactions.

Apart from that, the stellar initial mass function seems to be very universal, and the core mass function follows a similar slope. It is not clear what regulates the IMF, and what the connection to the core mass function is.

(Magneto-)hydrodynamical simulations of the star formation process can hardly include all the physics that is involved (e.g. detailed time-dependent heating/cooling), and lack the resolution to handle all relevant structures due to limited computing power. The latter hurdle is bypassed by sink particles, which form ad-hoc stars once 


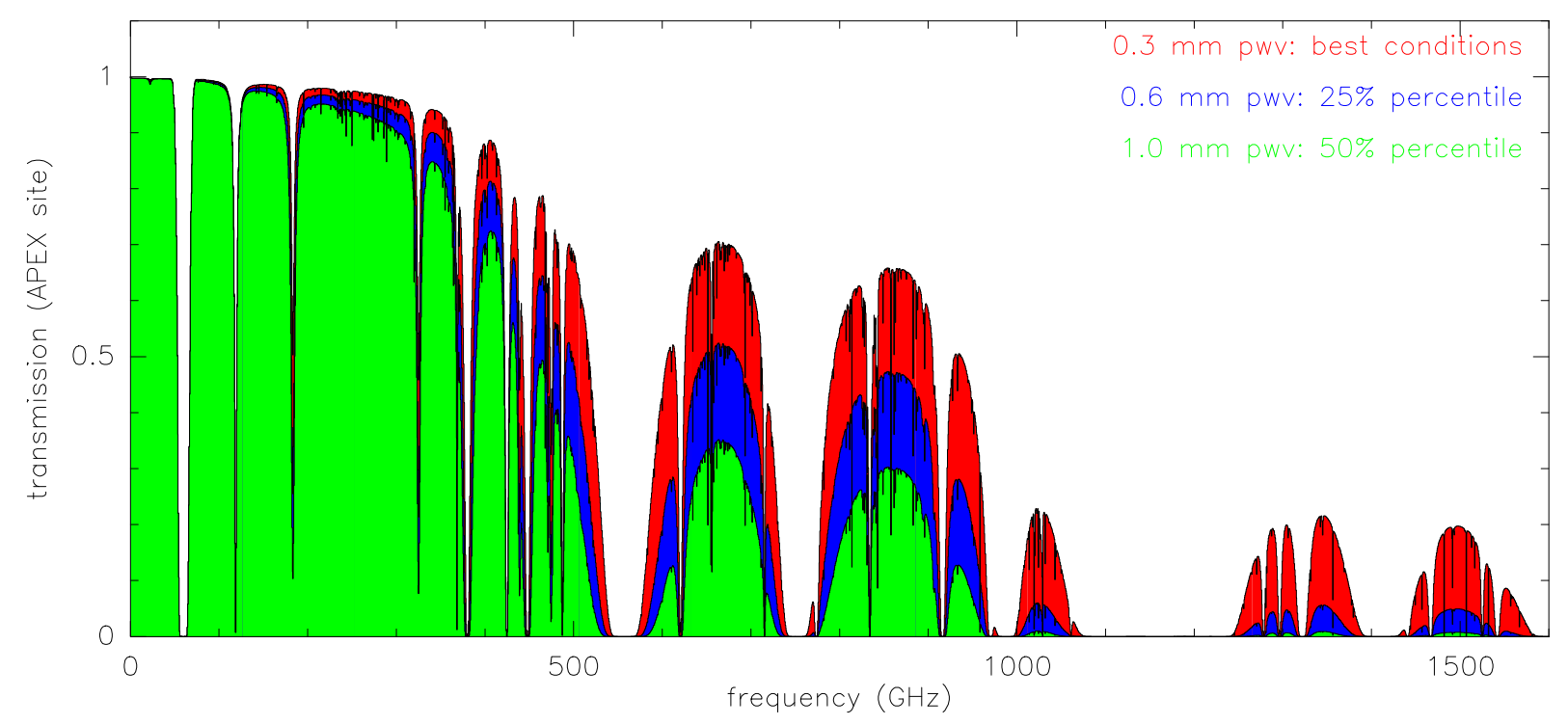

Figure 1.4: Atmospheric transmission at the APEX site, for columns of precipitated water vapor of $0.3,0.6$ and 1 mm (courtesy: P. Schilke).

the density gets too high. Still, even if the evolution is computed correctly, the outcome depends on the initial conditions, which have to be set a priori. It is not yet possible to compute several models, since one run takes several months to complete on the best available computers.

Astrochemical calculations need a complete network of reaction rates, as well as dust properties and timedependent physical conditions. The latter could come from hydrodynamical simulations.

\subsubsection{Observations}

High-mass stars are rare and undergo a fast formation and evolution process. Therefore, not many in a given stage of formation are present in the Galaxy, and they are mostly far away.

Moreover, the structures are very compact, and the cores from individual stars overlap due to the strong clustering of high-mass stars. Hence, very good angular resolution is a critical issue.

Due to the large dust column densities and the frequency-dependent dust opacity, most of the sources are obscured at short wavelengths. In the optical and infrared, one can only see a cold surface, while the hot core is hidden and only visible at submillimeter and radio wavelengths. These in turn are partly obscured by the Earth's atmosphere, in particular by water vapor. The transparency depends on weather and location. Figure 1.4 shows the transparency of the atmosphere as function of observing frequency for one of the best sites, under different weather conditions.

\subsubsection{Analysis}

The extreme richness of spectral lines that HMCs exhibit leads to spectral confusion and blending, i.e. line overlap. While this is less of a problem for strong lines (e.g. from simple molecules that have only relatively few transitions), it makes the detection of complex molecules, which have many weak transitions, very difficult and in many cases impossible, regardless of sensitivity. But also in the analysis of profiles of strong lines one has to be aware of possible blending by neighboring lines.

The individuality of the sources impedes a comparison to simulations, which have necessarily different initial conditions. This makes it difficult to test theories, which lack clear observational predictions.

The observed radiation depends on the physical structure, and it is not straightforward to derive physical and chemical quantities such as temperature, density, and molecular abundances. The abundances can vary with physical conditions, which renders the problem even more difficult.

For a proper analysis of the observations, one needs radiative transfer modeling. The models should be as simple as possible to allow a combination of parameters to reproduce the observations. Defining parameters and scanning the parameter space for solutions poses a giant challenge.

The modeling needs physical inputs, many of which are very uncertain or not yet measured or calculated. 
These include dust opacities, collision rates of molecules (collected at the database LAMDA ${ }^{2}$ and BASECOL ${ }^{3}$ ), and line frequencies (collected at the databases $\mathrm{CDMS}^{4}$ and $\mathrm{JPL}^{5}$ ).

\subsection{Aims}

The aim of this thesis is to constrain the structure of HMCs (their density, temperature, velocity, and abundance distribution) in order to facilitate future progress in high-mass star formation and in astrochemistry. This shall be achieved by exploring four ground-breaking techniques, two on the observational and two on the analytical side.

\subsubsection{High Submillimeter Frequencies}

High submillimeter frequencies ( $>600 \mathrm{GHz}$ ) are challenging due to atmospheric absorption (Fig. 1.4) and receiver technology. New instruments, namely APEX, the SMA, and Herschel, open up these frequency windows and provide the opportunity to use them in my research (Chapters 3, 4, and 6). Compared to lower frequencies, rotational transitions trace higher energy levels and the larger dust opacity facilitates absorption features, which are sensitive probes of the source structure. Also the continuum gives hints on the structure, as dust becomes optically thick.

\subsubsection{High Resolution}

High angular resolution requires a large aperture $D$, because the beam size (FWHM) is $252^{\prime \prime} \frac{\lambda[\mathrm{mm}]}{D[\mathrm{~m}]}$. As the wavelength $\lambda$ has to be long enough to penetrate the foreground dust, this means interferometers (see Sect. 2.5). Currently the best possible resolution provides the VLA in its most extended configuration and at its shortest wavelength (7 mm, Chapter 5). However, the brightness sensitivity decreases with higher resolution. At shorter wavelengths, where the dust emits stronger, the SMA can be used (Chapter 6). Spectral resolution is essential to measure line shapes, a resolution of around $1 \mathrm{~km} / \mathrm{s}$ is sufficient and provided by the heterodyne receivers.

\subsubsection{Modeling Line Shapes}

Not only the intensity of spectral lines contain important information, but lines have different optical depths at different velocities (frequency offsets from the rest frequency). While optically thin lines have usually a Gaussian shape, optically thick lines trace different parts of the source at different velocities. This offers access to the line-ofsight structure such as the excitation temperature profile and the velocity field (systematic motions like outflows, rotation, and infall) and can even constrain sub-beam size structures by detailed radiative transfer modeling, e.g. with RATRAN (Chapters 3 and 4).

\subsubsection{Three-dimensional Modeling}

Although models should be as simple as possible to reproduce the observations, real sources are not that simple. In particular, as high-resolution maps show, multiple cores and stars have formed, so spherical or cylindrical symmetry does not apply. Instead, one has to go for true three-dimensional models, at best in combination with computation of the dust temperature. This can be done with RADMC-3D (Chapters 5 and 6).

\footnotetext{
${ }^{2}$ http://www.strw.leidenuniv.nl/ moldata

${ }^{3}$ http://basecol.obspm.fr

${ }^{4}$ http://www.cdms.de

${ }^{5}$ http://spec.jpl.nasa.gov
} 


\section{Chapter 2}

\section{Radiative Transfer}

This chapter starts with brief descriptions of molecular energy levels and radiative transitions between them (Sects. 2.1 and 2.2). The determination of the level populations is discussed in Sect. 2.3. Section 2.4 deals with the resulting radiation. These sections are based on my diploma thesis (Rolffs 2007), and illustrated with examples from the HCN molecule ${ }^{1}$, which plays an important role in this thesis. The radiative transfer theory is also described e.g. in Rybicki \& Lightman (1979), Genzel (1991), and Rohlfs \& Wilson (2004).

How to measure the radiation with single-dish and interferometric telescopes is described in Sect. 2.5. Radiative transfer modeling is discussed in Sect. 2.6.

\subsection{Molecular Energy Levels}

\subsubsection{Rotational Levels}

The rotational energy of molecules has discrete levels due to the quantization of angular momentum $L$ (e.g. Haken $\&$ Wolf 2003). For linear molecules, it is given by

$$
E_{\mathrm{rot}}=\frac{L^{2}}{2 \Theta}=\frac{h^{2}}{8 \pi^{2} \Theta} J(J+1)
$$

with the moment of inertia $\Theta=\sum m_{i} R_{i}^{2}$ ( $m$ being the mass of a nucleus and $R$ its distance from the center of mass). $J$ is the quantum number of angular momentum. Its projection onto an arbitrary direction is another quantum number, whose possible values are $J, J-1, \ldots-J$. These $2 J+1$ projections have the same energy, so the energy level is degenerate and its statistical weight is $2 J+1$.

$\Theta$ depends on the equilibrium distances of the nuclei. These can be distorted by rotation or vibration. Due to centrifugal forces, $R$ and thus $\Theta$ increases with rotation, so high- $J$ states have a somewhat lower energy than they would have for constant $\Theta$. Including this effect, the energy levels can be approximated by $E_{\mathrm{rot}}=B J(J+1)-$ $D(J(J+1))^{2}$ with the molecular constants $B$ and $D$. Substituting one nucleus with a heavier isotope changes $\Theta$ and hence the rotational energy levels.

\subsubsection{Vibrational Levels}

Vibrational levels have higher energies than pure rotational levels. According to quantum mechanics, a harmonic oscillator has a vibrational energy proportional to $\left(v+\frac{1}{2}\right) \cdot v$ is the vibrational quantum number, and even for $v=0$ there are fluctuations which cause a substantial minimum vibrational energy (as the molecule cannot go lower in energy, it can be set to zero in practice). The proportionality constant depends on the force gradient and the (reduced) mass. For an anharmonic oscillator, a term proportional to $\left(v+\frac{1}{2}\right)^{2}$ and to the inverse of the dissociation energy has to be subtracted.

Different modes of vibration can be distinguished. Stretching vibrations are periodic changes of the bonding length, while bending vibrations change the angles between the bonds. The latter occur only for molecules with more than two atoms and usually require less energy. The energies depend on the atomic masses and the bonding length. An $\ell$-type finestructure arises due to the vibrational deformation of the molecule and the coupling between vibration and rotation.

\footnotetext{
${ }^{1}$ On Earth, HCN (hydrogen cyanide) is known as hydrocyanic or prussic acid (in German Blausäure) when it is dissolved in water. It is very poisonous: by reacting with iron of a certain enzyme, it blocks the respiration mechanism of cells and prevents the incorporation of oxygen from the blood. For prebiotic chemistry, it is important as a building block of amino acids.
} 
Electronic levels (the excitation of electrons) are generally so high in energy that they are not excited in starforming regions.

\subsubsection{Example: HCN Levels}

The molecular constants that describe the rotation of $\mathrm{HCN}$ are $B=1.478222 \mathrm{~cm}^{-1}=2.127 \mathrm{~K}=44.316 \mathrm{GHz}^{2}$ and $D=2.91 \times 10^{-6} \mathrm{~cm}^{-1}=4.2 \times 10^{-6} \mathrm{~K}=87.2 \mathrm{kHz}$. As the quadrupole moment of the ${ }^{14} \mathrm{~N}$ nucleus couples to the gradient of the electric field, a hyperfine splitting of the energy levels occurs. This is important only for the lowest rotational states. The 1-0 transition is split into 3 lines which differ by $3.5 \mathrm{MHz}\left(12 \mathrm{~km} \mathrm{~s}^{-1}\right)$, while the six $4-3$ transitions are distributed over $3 \mathrm{~km} \mathrm{~s}^{-1}$, with $95 \%$ of the line strength in the central transitions.

Vibrational modes of $\mathrm{HCN}$ are stretching of the $\mathrm{C}-\mathrm{N}$ or the $\mathrm{C}-\mathrm{H}$ bonds and bending of the $\mathrm{C}$ atom perpendicular to the $\mathrm{NH}$ line. The energy of the $\mathrm{C}-\mathrm{H}$ stretch vibrational mode is about $v_{1} \times 4764 \mathrm{~K}$ (the quantum number $v_{1}$ denotes the number of quanta in this mode), the $\mathrm{C}-\mathrm{N}$ stretch mode has $v_{3} \times 3017 \mathrm{~K}$ (less than $\mathrm{CH}$ due to the higher reduced mass). The designation ( 1 or 3 ) is sometimes reversed. Bending vibrations require $v_{2} \times 1027 \mathrm{~K}$.

While the stretching vibrations keep the linear structure of the molecule, the bending vibrations can occur in the plane of rotation or perpendicular to it, so the bending mode is actually split into two, slightly different modes. If the orientation of each of the $v_{2}$ quanta is + or - , the sum is the quantum number $\ell$, which therefore goes from $v_{2}$ to $-v_{2}$ in steps of 2 . $\ell$ couples rotation and vibration, and is actually another rotational quantum number: Due to the bending deformation, rotation around the axis becomes possible. The result of the two simultaneous rotations is that $J$ cannot be smaller than $\ell$ and that a bending mode of quantum number $v_{2}$ splits into $v_{2}+1$ levels, whose energies depend mainly on the absolute value of $\ell$ and slightly on its sign (the direction of rotation), which is $(-1)^{J}$ for the lower levels. An alternative designation is $e$ for the lower levels (and the ground vibrational state) and $f$ for the upper levels.

The energy level diagram of HCN is summarized in Figure 2.1 (based on Maki 1974; Maki et al. 2000).

\subsection{Radiative Transitions}

\subsubsection{Einstein Coefficients}

The probabilities for radiative transitions are described by the Einstein Coefficients (Einstein 1916). Considering two levels, the populations $n_{u}$ and $n_{l}$ (number densities of upper and lower level, respectively) change according to

$$
\frac{d n_{u}}{d t}=-\frac{d n_{l}}{d t}=-A_{u l} n_{u}-B_{u l} J_{u l} n_{u}+B_{l u} J_{u l} n_{l}
$$

$n_{u}$ can decrease by spontaneous emission, which is proportional to $n_{u}$ itself and to the $A$ coefficient. The $B$ coefficients describe transitions induced by radiation. The probabilities are proportional to the mean intensity $J_{u l}$, which is the intensity from all directions weighted by the profile function $\Phi$ (Sect. 2.4.4; it is centered at $v_{u l}=\frac{E_{u}-E_{l}}{h}$, where $E$ denotes the energy), $J_{u l}=\int \frac{d \Omega}{4 \pi} \int d v I \Phi$. Induced emission, described by the second term, means that a passing photon of the right frequency is doubled. This process is thus proportional to $n_{u}$. The last term describes the absorption of a passing photon, which causes the transition from the lower to the upper level.

The Einstein $B$ coefficients are proportional to the $A$ coefficient (Sect. 2.3.2). The strength (Einstein $A$ coefficient) of a transition is calculated quantum-mechanically from the wave functions of the two states. For electric dipole transitions, it is proportional to $\mu^{2} v^{3}$ with the dipole moment $\mu$. For forbidden (multipole) transitions, it is very low. Hence, radiative transitions are unlikely if they violate certain selection rules, and are more likely the larger the energy gap is. The selection rules describe the conditions for allowed (strong) transitions. For instance, $\Delta J=1$ is necessary for a rotational transition, since the photon carries one quantum of angular momentum.

\subsubsection{Example: HCN Transitions}

Due to the corresponding energy gaps, direct $\ell$-type transitions occur in the radio regime, rotational transitions in the $\mathrm{mm}$ to far-infrared regime, vibrational transitions in the infrared $\left(14 \mu \mathrm{m}\right.$ for $\left.\Delta v_{2}=1\right)$ and electronic transitions in the ultraviolet (190 $\mathrm{nm}$ for the electronic state of $\mathrm{HCN}$ ).

The selection rules are bit more complicated when the vibrational levels are included. Pure rotational transitions stay within a certain vibrational state. Rovibrational transitions (which change the vibrational state) require $\Delta J=1$ for the lower level of an $\ell$-type doublet and $\Delta J=0$ for the upper level. Direct $\ell$-type transitions are not electric dipole transitions and hence weak. They exchange upper and lower level of the same $J$ with $\Delta \ell=2$.

\footnotetext{
${ }^{2}$ All units denote the energy, making use of $E=k T=h v=\frac{h c}{\lambda}$
} 

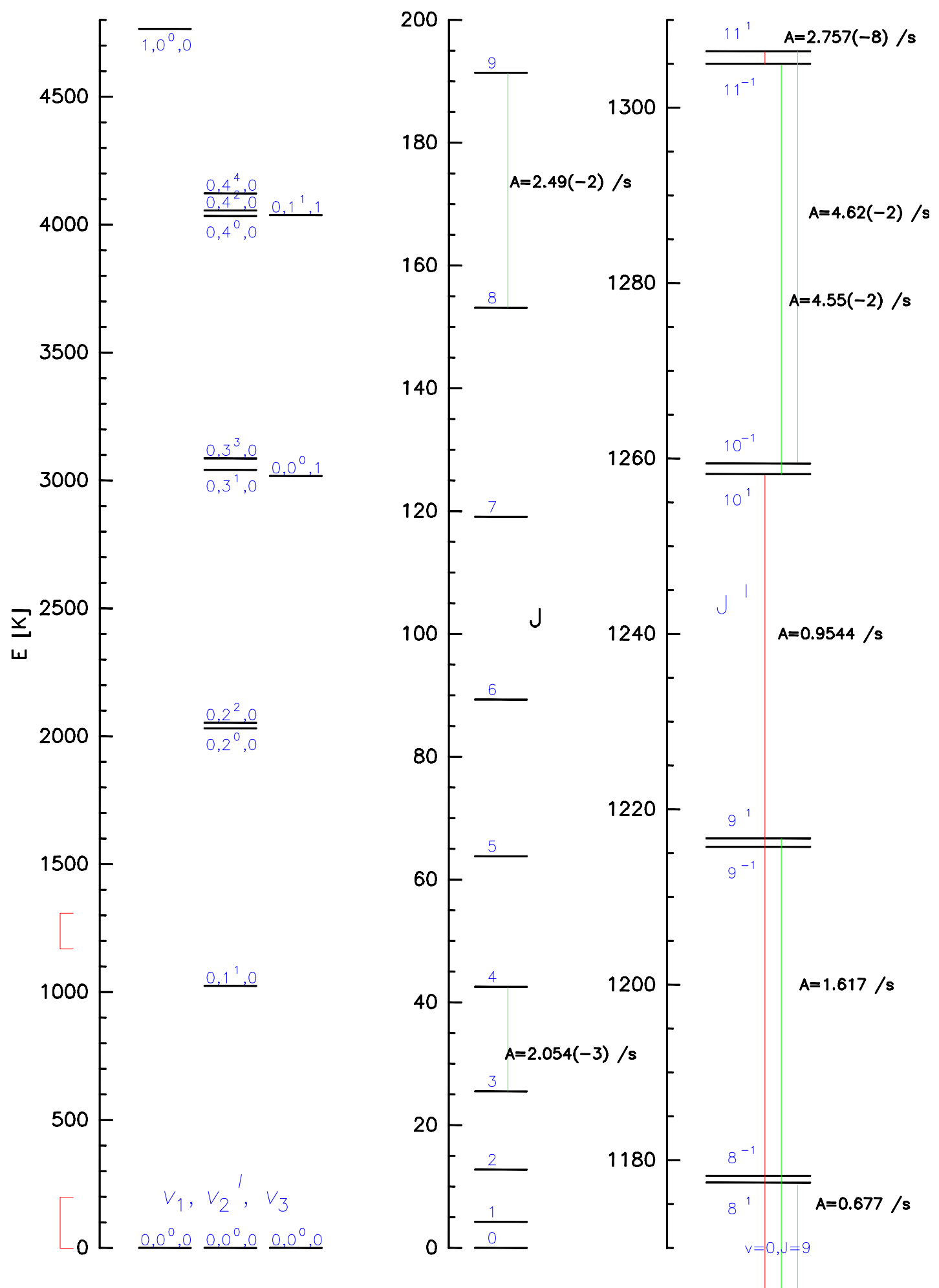

Figure 2.1: Energy levels of HCN. The left panel shows the vibrational levels, the central panel the lowest rotational levels and the right panel shows levels of the first vibrational state. Some transitions with their Einstein A coefficient (numbers in parenthesis are powers of 10) are indicated. 
If an HCN molecule is in the upper level of a certain $J$ in $v_{2}=1$, the most likely transition is to $J$ in $v=0$, the next likely is to the upper level of $J-1$ in $v_{2}=1$, and an unlikely transition is to the lower level of the same $J$. If it is in the lower level, it can decay to $J-1$ or $J+1$ in $v=0$ or to the lower level of $J-1$ in $v_{2}=1$. In the right panel of Figure 2.1, some transitions with their Einstein $A$ value are indicated. All transitions relevant for astronomy can be found in the Cologne Database for Molecular Spectroscopy (CDMS, Müller et al. 2001, 2005).

$\mathrm{HCN}$ in space was first detected by Huebner et al. (1974) in a comet through its 1-0 transition. Vibrationally excited HCN was detected by Ziurys \& Turner (1986) towards Orion-KL and the carbon star IRC+10216. Also in Orion-KL the $J=9-8$ transition was first detected (Stutzki et al. 1988). In the same carbon star, many vibrational states can be observed, and the 9-8 transition in $v_{2}=4, \ell=0$ shows maser emission due to Coriolis coupling with the $v_{3}=1, v_{2}=1$ state (Schilke et al. 2000). Direct $\ell$-type transitions were detected by Thorwirth (2001) toward the protoplanetary nebula CRL 618 (Thorwirth et al. 2003b) as well as Orion-KL, SgrB2-N and G10.47.

\subsubsection{The Planck Function}

Quantum Statistics yields an expression for the radiation field, the Planck formula $B$. The derivation is based on the insight that photons are bosons and on the evaluation of the density of states. The expression is

$$
B=\frac{2 h v^{3}}{c^{2}}\left(e^{\frac{h v}{k T}}-1\right)^{-1}
$$

Although discrete energy levels were assumed in the derivation, this formula describes both line and continuum radiation: In a solid body the energy levels are so close together that every frequency is possible, and so it emits continuous thermal radiation. The emitted radiation depends on the optical depth, which is again frequencydependent (Sect. 2.4).

The Planck function describes the radiation that every body emits due to its temperature. The wavelength of maximum emission is $\lambda_{\max }=29 \mu \mathrm{m} \frac{100 \mathrm{~K}}{T}$ (Wien's displacement law), and the total emission ( $B$ integrated over frequency and solid angle) is proportional to $T^{4}$ (Stefan-Boltzmann law).

If $h v \ll k T$, the Rayleigh-Jeans approximation $e^{\frac{h v}{k T}} \approx 1+\frac{h v}{k T}$ is valid:

$$
B_{\mathrm{RJ}}=\frac{2 h v^{3}}{c^{2}} \frac{k T}{h v}=\frac{2 v^{2} k T}{c^{2}}
$$

As here $B \propto T$, one can express an intensity $I$ as a temperature $T_{\mathrm{b}}$ (brightness temperature). Even if the RayleighJeans approximation is not valid, the brightness temperature is defined as

$$
T_{\mathrm{b}}=\frac{c^{2}}{2 k v^{2}} I
$$

If a source had infinite extension and optical depth and a uniform temperature, then this temperature would be the brightness temperature in the limit of low frequencies, while at higher frequencies $T_{\mathrm{b}}$ would be less than the temperature. For a real source, the telescope's finite resolution causes angular averaging (Sect. 2.5), and along the line of sight $T_{\mathrm{b}}$ is averaged according to the temperature structure and the optical depth (Sect. 2.4).

\subsection{Level Populations}

\subsubsection{Collisional Transitions}

In addition to radiative transitions, it is also possible to change the state by collisions. The probability for such transitions is proportional to the number of collisions, therefore to the density of collision partners $n_{\text {col }}$ times the density of the molecule. For a two-level-system, this is described by the following equation:

$$
\frac{d n_{u}}{d t}=-\frac{d n_{l}}{d t}=-\gamma_{u l} n_{\mathrm{col}} n_{u}+\gamma_{l u} n_{\mathrm{col}} n_{l}
$$

The rate coefficients $\gamma_{u l}$ and $\gamma_{l u}$ (from upper to lower and from lower to upper level, respectively) depend on the levels, the molecule and the collision partner. Their ratio is determined by the temperature (Sect. 2.3.2, equation 2.9).

In molecular clouds, the collision partners are molecular hydrogen (6 out of 7 particles) and Helium atoms (1 out of 7, 25\% by mass). In many cases, considerable differences for collisions with ortho- and para- $\mathrm{H}_{2}$ exist (e.g. Flower \& Launay 1985; Offer \& van Dishoeck 1992). Other particles (electrons, ions, dust particles, CO and other molecules, $\mathrm{H}$ and other atoms) are rare compared to $\mathrm{H}_{2}$ and $\mathrm{He}$, but the cross sections for collisions with electrons 
(abundance around $10^{-7}$ ) is large, particularly for ions. Usually $n_{\text {col }}$ is set equal to the $\mathrm{H}_{2}$ density and the rate coefficients are scaled to include Helium and electrons.

Merging equations (2.2) and (2.6) and generalizing to a multi-level-system yields for every state $i$

$$
\frac{d n_{i}}{d t}=\sum_{j>i}\left(n_{j} A_{j i}+\left(n_{j} B_{j i}-n_{i} B_{i j}\right) J_{i j}\right)-\sum_{j<i}\left(n_{i} A_{i j}+\left(n_{i} B_{i j}-n_{j} B_{j i}\right) J_{i j}\right)+\sum_{j} n_{\mathrm{col}}\left(n_{j} \gamma_{j i}-n_{i} \gamma_{i j}\right)
$$

When statistical equilibrium is reached, the level populations stay the same, $\frac{d n_{i}}{d t}=0$ for every $i$ (although the individual molecules change their state). For given conditions (density, gas temperature, and radiation field), the level populations are determined by the above equation. As the radiation field in turn depends on the level populations, this is a non-linear equation.

\subsubsection{Boltzmann Distribution}

A huge simplification compared to the solution of equation (2.7) arises for the case of Local Thermodynamic Equilibrium (LTE, Sect. 2.3.3). Statistical Thermodynamics predicts for an equilibrium system of temperature $T$ (canonical ensemble) that the levels are populated according to the Boltzmann distribution:

$$
\frac{n_{u}}{n_{l}}=\frac{g_{u}}{g_{l}} e^{-\frac{E_{u}-E_{l}}{k T}} \quad \text { or } \quad \frac{n_{i}}{n_{t o t}}=\frac{g_{i}}{Q} e^{-\frac{E_{i}}{k T}}
$$

The statistical weight $g$ is the degeneracy of a level, i.e. how many quantum states the energy level has. $E$ is the energy above ground, $n_{t o t}$ is the number density of the molecule and $Q=\sum_{i} g_{i} e^{-\frac{E_{i}}{k T}}$ is the partition function that accounts for the condition $\sum_{i} n_{i}=n_{t o t}$.

Einstein coefficients and collisional rate coefficients are intrinsic properties of a certain transition of a certain molecule or atom. They do not depend on environmental conditions. Therefore, if a statement on them is valid under such a condition, it is generally valid.

If the density $n_{\mathrm{col}}$ is high enough, the radiative terms can be neglected and equation (2.6) in steady state determines the level populations by $n_{u} \gamma_{u l}=n_{l} \gamma_{l u}$. With the Boltzmann equation (2.8), the rate coefficients are related as

$$
\frac{\gamma_{l u}}{\gamma_{u l}}=\frac{g_{u}}{g_{l}} e^{-\frac{E_{u}-E_{l}}{k T}}
$$

So if one collisional rate coefficient is known, the other one is given according to the temperature.

Similarly, if the radiation field is strong enough, the collisional terms can be neglected and equation (2.2) in steady state can be written as $J_{u l}=\frac{n_{u} A_{u l}}{n_{l} B_{B_{u}}-n_{u} B_{u l}}$. With the radiation field given by the Planck formula (2.3) and inserting the Boltzmann equation (2.8), this reads

$$
B=\frac{n_{u} A_{u l}}{n_{l} B_{l u}-n_{u} B_{u l}}=\frac{A_{u l}}{\frac{g_{l}}{g_{u}} e^{\frac{E_{u}-E_{l}}{k T}} B_{l u}-B_{u l}}
$$

Comparison with $B$ (equation 2.3) yields the relation between the Einstein coefficients:

$$
\begin{gathered}
g_{u} B_{u l}=g_{l} B_{l u} \\
A_{u l}=\frac{2 h v^{3}}{c^{2}} B_{u l}
\end{gathered}
$$

\subsubsection{Local Thermodynamic Equilibrium (LTE) and Beyond}

If the level populations are uniquely determined by the temperature, LTE reigns. This is generally the case if the density is high enough that radiative transitions are negligible compared to collisional transitions, or if a thermal radiation field is strong enough. The radiation field can also consist of line photons which are trapped due to high optical depth: In this case a radiative decay leads to excitation of another molecule, so the energy of a collision is not lost, but just transfered to another place.

The Boltzmann distribution is only maintained if the spontaneous decay, that tends to drive the molecules into the lowest energy level, is compensated by excitation. If the excitation is insufficient, the upper level will depopulate, reaching a new equilibrium. Imitating the Boltzmann distribution, an arbitrary ratio of upper and lower level can be described by an excitation temperature, defined as

$$
T_{\mathrm{ex}}(u-l)=-\frac{E_{u}-E_{l}}{k \ln \left(\frac{n_{u} g_{l}}{n_{l} g_{u}}\right)}
$$


In LTE, the excitation temperature of all transitions is the kinetic temperature.

Usually, collisions excite the upper levels, which decay radiatively or collisionally. Thus, kinetic energy is transformed to potential energy, and the gas cools by radiative losses. If the collisional excitation is frequent enough, it can thermalize the level populations, and the excitation temperature is the kinetic temperature. If it occurs too rarely, however, the upper level is less populated than in LTE and the excitation temperature is less than the kinetic temperature. This happens roughly if the density $n_{\text {col }}$ is less than the critical density $n_{\text {crit }}$, at which the change of the level populations by spontaneous emission $A_{u l} n_{u}$ is as strong as by collisions $\gamma_{u l} n_{\mathrm{col}} n_{u}$ :

$$
n_{\mathrm{crit}}=\frac{A_{u l}}{\gamma_{u l}}
$$

The critical density is different for every transition, and for $n_{\text {col }} \ll n_{\text {crit }}$ the thermal ratio of $n_{u} / n_{l}$ (equation 2.8 ) is multiplied by $n_{\text {col }} / n_{\text {crit }}$, resulting in very weak emission.

The radiation field as well can thermalize the level populations, if it is optically thick and comes from all directions: Neglecting collisions one has from equation (2.2) $-\left(A_{u l}+B_{u l} J_{u l}\right) n_{u}+B_{l u} J_{u l} n_{l}=0$ or $\frac{n_{u}}{n_{l}}=\frac{B_{l u} J_{u l}}{A_{u l}+B_{u l} J_{u l}}$. Substituting the Planck function (2.3) and the Einstein coefficients (2.12) and (2.11), the Boltzmann distribution is recovered. For the general case, the Planck function has to be multiplied by $1-e^{-\tau}$ and the fraction of the sky that is covered by the source, so the upper level will have a lower population.

In the case of radiative excitation, the radiation temperature would determine the excitation temperature, while it is the kinetic temperature in the case of collisional excitation. These two temperatures are not necessarily equal, e.g. the dust can be heated by external radiation to which the gas is transparent, or the gas can be heated by shocks while dust remains cool. Very high densities would provide enough collisions to adjust gas and dust temperatures, and also guarantee that one single temperature would describe the level populations, but for most interstellar conditions there is no way out: The complicated equation (2.7) has to be solved for a given density, kinetic temperature and radiation field to determine the level populations and thus the line radiation.

\subsubsection{Non-LTE Methods}

The level populations depend on the radiation, which in turn depends on the level populations. If the density is so high that collisions can excite all levels faster than they decay, one can simply use the Boltzmann equation (2.8) and avoid this problem. However, LTE is generally not the case, and one has to use the rate equations (2.7) for statistical equilibrium.

A method to solve these equations is the $\Lambda$ iteration. The LTE populations give a source function (Sect. 2.4.1), and the $\Lambda$ operator computes the mean intensities $J_{i j}$ for each cell and transition from the source functions of all cells. It thus describes the local response of the radiation field to perturbations of the level populations. With the $J_{i j}$, new level populations are obtained using (2.7), which change the $\Lambda$ operator, and the procedure is repeated until the population changes are low enough.

The convergence of the $\Lambda$ iteration can be accelerated by splitting the $\Lambda$ operator into a local and an external contribution (Accelerated Lambda Iteration ALI, Rybicki \& Hummer 1991). The local part, which contains the radiation emitted by the cell itself (or by the cell and its nearest neighbors), is the diagonal (or tridiagonal) part of the $\Lambda$ matrix and can easily be inverted. This direct inversion allows to solve the local problem without iteration and removes self-coupling within a cell. The external radiation acts as a perturbation to the level populations, which have to be solved iteratively. Especially for high optical depths the convergence is much faster this way.

The Monte-Carlo method (Bernes 1979) sends photons of random direction and frequency through the medium. They interact with the matter and change the level populations. The number of photons has to be sufficiently large for a good sampling of directions and frequencies and for a low statistical error.

Similarly to the ALI method, the convergence of Monte Carlo can be accelerated by splitting the radiation field into local and external mean intensities (Accelerated Monte Carlo, Hogerheijde \& van der Tak 2000). The local field simplifies emission and absorption within the same cell by using local source function and opacity, which are given by the current level populations. The field is obtained by summing up rays between random positions on the boundary and within the cell $\left(S\left(1-e^{-\tau}\right)\right)$, and adding the diluted external field $\left(I_{0} e^{-\tau}\right)$ for each ray direction. Without this acceleration, optically thick cells would trap photons in random walks (permanent absorption and re-emission).

Several approximations are based on an escape probability mechanism (EP) to simplify the non-locality of the problem. An emitted photon has a certain probability to escape the source, in which case it does not contribute to the excitation. For a very low optical depth, the escape probability may be unity, and the level populations at different locations do not influence one another.

In the case of a monotonous velocity field with a large velocity gradient (LVG, Sobolev 1960), different regions have different velocities, so the photons are Doppler-shifted and the profile functions do not overlap. The region of 


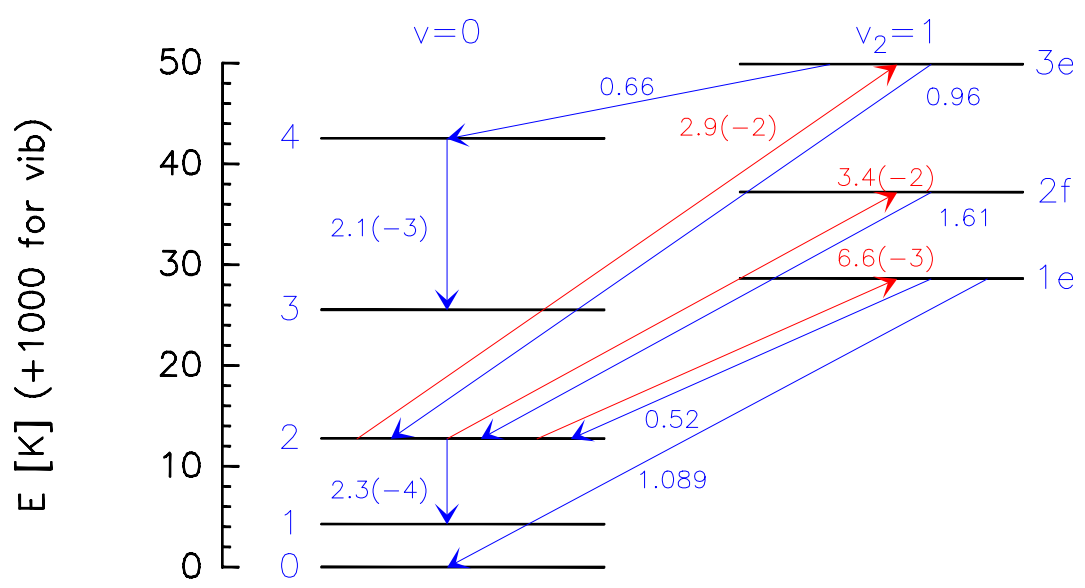

Figure 2.2: Infrared pumping leading to population of $J=4$ from $J=2$. All radiative transitions from $J=2$ are drawn (blue downward, red upward). The rates (in 1/s), written next to the lines, are the Einstein $A$ coefficients for the downward transitions and $B_{l u} J_{14 \mu \mathrm{m}}$ for the upward transitions, where surrounding dust of $300 \mathrm{~K}$ and $\tau_{14 \mu \mathrm{m}}=1$ was assumed. Lower temperature, $\tau$ or sky covering fraction significantly weakens the IR excitation. $A_{9,8}$ is with $2.5 \times 10^{-2} / \mathrm{s}$ as large as the the rate to $v_{2}=1, J=10$, so IR pumping would stop to be efficient near $J=9$ (but at frequencies of these rotational transitions, the dust continuum is strong enough that direct pumping becomes important, and also higher vibrational states contribute).

interaction depends on the velocity gradient and the line width. The LVG method allows to rapidly compute level populations by ignoring non-local effects.

The Coupled Escape Probability method (CEP, Elitzur \& Asensio Ramos 2006) couples the different cells to get the exact solution. The problem is transformed to solving only algebraic equations.

Several methods are described and compared in van Zadelhoff et al. (2002) and in Pavlyuchenkov et al. (2007). An application of an ALI method can be found e.g. in Keto et al. (2004)

\subsubsection{Example: HCN Excitation}

HCN has a high critical density (a few times $10^{6} \mathrm{~cm}^{-3}$ for $1-0$, more than $10^{8} \mathrm{~cm}^{-3}$ for $4-3$, and more than $10^{9}$ $\mathrm{cm}^{-3}$ for 9-8). This means that collisions cannot excite it sufficiently when the density is not very high. At low densities, each collision is followed by radiative decay, resulting in a large population of $J=0$ and very few molecules in higher- $J$ states.

Another way to excite the molecule is radiative excitation. Line trapping occurs when a strong line, originating from a high-density region, excites other molecules, whose line emission can in turn excite other molecules. The line radiation can only get lost when the optical depth is low enough. This is a non-local effect and transfers high excitation from high to low densities.

Collisional excitation of the vibrational states requires extremely high densities, which are not found in the ISM. Warm dust emits strong infrared (IR) radiation, though, which can excite HCN. If the radiation field is strong enough, the vibrational level populations thermalize to the dust temperature.

As the Einstein $A$ values of the IR transitions are high, a vibrationally excited molecule quickly falls back to the ground state. Because $\Delta J=1$ (or 0 ) for both transitions, the original $J$ can change by 2 or stay the same. Radiative excitation to $J+1$ is more likely than to $J-1$ because of the statistical weight, of which the decay is independent. The result is that even without collisional excitation, the whole $J$ ladder is populated until spontaneous rotational decay $\left(A_{J, J-1}\right)$ becomes larger than the excitation to the vibrational state $\left(B_{v=0, v_{2}=1} J_{14 \mu \mathrm{m}}\right)$. This IR pumping (Carroll $\&$ Goldsmith 1981; Hauschildt et al. 1993) accounts for part of the populations in the higher- $J$ states. It is illustrated in Figure 2.2.

\subsection{Optical Depth}

\subsubsection{Basic Radiative Transfer}

For the definition of the intensity of electromagnetic radiation one can imagine the following: A ray direction and a frequency are given. Now one counts all $N$ photons within a frequency interval $d v$ that fly through a plane $d A$ (perpendicular to the ray direction) and that go to (or come from) a solid angle $d \Omega$ (in the ray direction). As $N$ is 
proportional to $d v, d A, d \Omega$ and to the measurement time $d t$, the intensity $I$ is defined as the energy of the radiation divided by these parameters:

$$
I=\frac{N h v}{d t d v d A d \Omega}
$$

While photons of frequency $v$ travel a length $d s$, they can be absorbed or scattered to another direction by the matter at this location, which in turn can emit photons or scatter them to our direction. However, scattering can be completely neglected at wavelengths longer than near-infrared and will be ignored here.

As the decrease of the intensity is proportional to the intensity itself (Lambert-Beer law), while the increase is usually independent of it, the intensity change can be split into these two parts:

$$
d I=-\kappa I d s+\epsilon d s
$$

The absorption coefficient or opacity $\kappa$ and the emission coefficient $\epsilon$ specify the probability that a photon is absorbed and emitted, respectively. They depend on the frequency and the characteristics and density of the matter. Stimulated emission is included in $\kappa$ as 'negative absorption'.

The optical depth $\tau$ is differentially defined as $d \tau=-\kappa d s$, so it weights the line-of-sight according to its absorption capability and reverses its direction: $\tau=-\int_{s_{0}}^{s} \kappa d s=\int_{s}^{s_{0}} \kappa d s$ is 0 at the observer's location $s_{0}>s$. The basic equation of radiative transfer (2.16) can thus be written as

$$
\frac{d I}{d \tau}=I-S
$$

with the source function $S=\frac{\epsilon}{\kappa}$. Multiplying this equation by $e^{-\tau}$, it can be transformed to $-S e^{-\tau}=\frac{d I}{d \tau} e^{-\tau}-$ $I e^{-\tau}=\frac{d}{d \tau}\left(I e^{-\tau}\right)$. Integration from 0 to $\tau$ yields

$$
I(\tau) e^{-\tau}-I(0)=-\int_{0}^{\tau} S e^{-\tau^{\prime}} d \tau^{\prime}
$$

$I(\tau)=I_{0}$ is the background intensity at $s$, and $I(0)=I$ is the intensity at the observer's location $s_{0}$. If $S$ is independent of $\tau$ (homogeneous medium or sufficiently small distance), solving the integral gives

$$
I=I_{0} e^{-\tau}+S\left(1-e^{-\tau}\right)
$$

In the optically thin case $(\tau \ll 1)$ the intensity is $I=I_{0}(1-\tau)+S \tau$, so part of the background is substituted by the foreground. In the optically thick case $(\tau \gg 1), I=S$, so one sees only the foreground. As $\tau$ is highly frequency-dependent, different layers can be seen at different frequencies.

\subsubsection{Dust Opacity}

Interstellar dust grains are the more abundant, the smaller they are (Mathis et al. 1977). A consequence of the size distribution of dust grains and their properties of electromagnetic interaction is that emission and absorption is generally stronger the shorter the wavelength is. This can be described by $\kappa_{\text {dust }} \propto \gamma^{\beta}$ with $\beta$ between 1 and 2 , plus some maxima at resonance frequencies of graphites and silicates.

Exact shape and magnitude of the opacity depend on the type of grains, however, and differ a lot. For example, the grains can coagulate to larger grains (for very large grains, $\beta \approx 0$ ), and gas can freeze out and form ice mantles around them. The dust opacity is thus dependent on density, temperature, elemental abundances and evolution (coagulation time); it is therefore complicated and very uncertain. As the opacity is proportional to the mass density of dust, it is usually given as $\kappa_{\text {dust }} / \rho_{\text {dust }}$ (e.g. in Ossenkopf \& Henning 1994). The total optical depth is proportional to the column density of $\mathrm{H}_{2}$ (if the gas is molecular):

$$
\tau_{\text {dust }}=N_{\mathrm{H}_{2}}\left[\mathrm{~cm}^{-2}\right] \frac{\kappa_{\text {dust }}\left[\mathrm{cm}^{-1}\right]}{\rho_{\text {dust }}\left[\mathrm{g} \mathrm{cm}^{-3}\right]} \frac{\rho_{\text {dust }}}{\rho_{\text {gas }}} 4.55 \times 10^{-24}
$$

with $\frac{\rho_{\text {dust }}}{\rho_{\text {gas }}} \approx \frac{1}{100}$. When $73 \%$ of the gas mass is in the form of $\mathrm{H}_{2}(25 \%$ Helium and $2 \%$ metals $)$, the gas mass per $\mathrm{H}_{2}$ molecule is two proton masses divided by 0.73 , which is $4.55 \times 10^{-24} \mathrm{~g}$.

For dust, $\tau$ generally rises with frequency. It decreases for ionized gas, as to produce free-free radiation, an electron changes its velocity vector in the field of a nucleus, which happens more often for small changes. For a star, $\tau$ is large for all frequencies, but one still sees different layers at different frequencies. For line radiation, as the name suggests, $\tau$ has a narrow peak at the transition frequency $v_{u l}=\frac{E_{u}-E_{l}}{h}$. 


\subsubsection{Line Width}

As the transition occurs between discrete energy levels, one may wonder how a frequency different from $v_{u l}$ is possible at all. There are five reasons for the line width:

- Natural Broadening: Most fundamental, the Heisenberg uncertainty relation puts a limit on the accuracy of energy $\Delta E$ and time $\Delta t: \Delta E \Delta t \geq \frac{h}{4 \pi}$. For a transition, the time uncertainty is given by the statistical lifetime of the upper level, $\Delta t=1 / A_{u l}$, and causes the photon to have an energy uncertainty of $\Delta E=h \Delta v$. Therefore, the natural line width is roughly the Einstein $A$ coefficient and very small.

- Pressure Broadening: When the molecule is colliding, its energy levels are distorted because of interaction with the collision partner. This strongly depends on the pressure, which measures the amount and strength of collisions. For the low interstellar densities, this pressure broadening is generally not important.

- Thermal Broadening: The radial velocity v (projection to the line of sight, positive for receding and negative for approaching motion) causes a Doppler shift in the frequency, from the rest frequency $v_{0}$ to the sky frequency $v$ :

$$
\frac{\mathrm{v}}{c}=\frac{v_{0}-v}{v_{0}}
$$

The individual velocities of the molecules follow a Maxwell distribution, with the average speed proportional to $\sqrt{\frac{T}{m}}$ ( $m$ being the molecular mass, thermal energy is kinetic energy of the particles). This results in a thermal line width of $\Delta \mathrm{v}=\sqrt{\frac{k T}{m}}$ (related to frequency by $\left.\frac{\Delta \mathrm{v}}{c}=\frac{\Delta v}{v}\right)$. For $m=27 \times 1.67 \times 10^{-27} \mathrm{~kg}(\mathrm{HCN})$ and $T=300 \mathrm{~K}$, it is $0.3 \mathrm{~km} \mathrm{~s}^{-1}$.

- Turbulent Broadening: Macroscopic motions of the gas shift the frequency according to equation (2.21). On the one hand, the whole source moves relative to us, shifting all lines. This velocity is given as $\mathrm{v}_{\mathrm{LSR}}$, the velocity relative to the Local Standard of Rest. On the other hand, internal motions such as turbulence smear out the lines

- Systematic Motions: While turbulent motion is chaotic and undirected, systematic motions such as outflows, rotation, infall, and different systemic velocities of different parts of the cloud cause additional line broadening. Turbulence and systematic motions combined result in line widths of $3-15 \mathrm{~km} \mathrm{~s}^{-1}$ for the sources of this work.

The frequency dependence of $\tau, \kappa$ and $\epsilon$ is described by the profile function $\Phi_{u l}(v)$. Its integral is normalized to unity, $\int_{0}^{\infty} \Phi d v=1$, so it distributes the absorption/emission strength over the frequencies.

The simplest approximation for $\Phi$ is a box centered at $v_{u l}$ with a width $\Delta v$, the line width, and a height of $1 / \Delta v$. For thermal broadening, it is a Gaussian function:

$$
\Phi_{u l}=\sqrt{\frac{4 \ln 2}{\pi}} \frac{1}{\Delta v} e^{-\frac{4 \ln 2\left(v-v_{u l}\left(1-\frac{v}{c}\right)\right)^{2}}{\Delta v^{2}}}
$$

For natural and pressure broadening, it is a Lorentzian function (or Breit-Wigner distribution):

$$
\Phi_{u l}=\frac{1}{2 \pi} \frac{\Delta v}{\left(v-v_{u l}\left(1-\frac{\mathrm{v}}{c}\right)\right)^{2}+\Delta v^{2} / 4}
$$

$\Delta v$ is the Full Width at Half Maximum, FWHM, and the central frequency $v_{u l}$ can be shifted by the relative radial velocity v (equation 2.21). For macroscopic motions, $\Phi$ can be arbitrarily complicated. It is usually assumed to be a Gaussian with turbulence dominating. In more general cases, it can be taken as a Voigt function (convolution of Gaussian and Lorentzian) with certain local velocity and line width(s).

Now the exact expressions for the absorption coefficient $\kappa$ and the emission coefficient $\epsilon$ can be given. Comparing equations (2.16) and (2.2), one sees that $\epsilon \propto n_{u} A_{u l}$ and $\kappa \propto n_{l} B_{l u}-n_{u} B_{u l}$, as the decrease of $n_{u}$ goes into increase of the intensity. $\epsilon$ has the unit of intensity per length, i.e. energy per volume, time, solid angle and frequency (see equation 2.15). $n_{u} A_{u l}$ is the number of photons per time and volume, so it has to be multiplied by $h v$ (energy), $1 / 4 \pi$ (solid angle, all directions) and $\Phi$ (frequency). The same applies to $\kappa$, because $B_{u l} I$ has the unit of $A_{u l}\left(\mathrm{~s}^{-1}\right)$.

$$
\begin{gathered}
\epsilon=\frac{h v}{4 \pi} n_{u} A_{u l} \Phi \\
\kappa=\frac{h v}{4 \pi}\left(n_{l} B_{l u}-n_{u} B_{u l}\right) \Phi=\frac{c^{2}}{8 \pi v^{2}} n_{u} A_{u l} \Phi\left(e^{\frac{h v}{k T_{\mathrm{ex}}}}-1\right)
\end{gathered}
$$




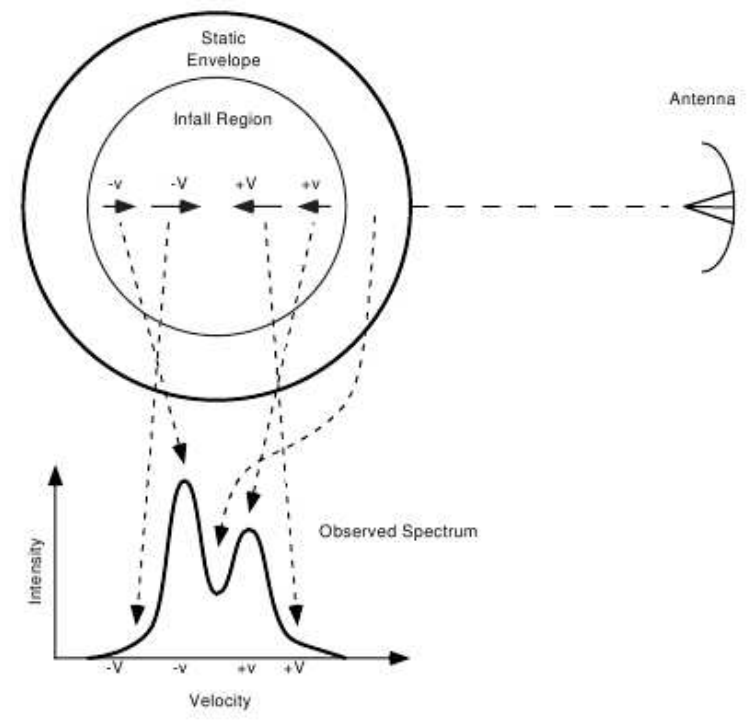

Figure 2.3: Infall profile, resulting from velocity-selective absorption. Inside-out collapse is shown, but other inward motions produce very similar line shapes. Figure from Evans (2003)

In the last step, the relations between the Einstein coefficients (2.11 and 2.12) and the definition of the excitation tempertature (2.13) were used. The source function is given by

$$
S=\frac{\epsilon}{\kappa}=\frac{n_{u} A_{u l}}{n_{l} B_{l u}-n_{u} B_{u l}}=\frac{2 h v^{3}}{c^{2}}\left(e^{\frac{h v}{k T \mathrm{ex}}}-1\right)^{-1}
$$

which is the Planck function (2.3) at the excitation temperature. $\kappa$ and $\epsilon$ are generally sums of different components, e.g. contributions from lines that can partially overlap and from the dust continuum.

\subsubsection{Line Profiles}

The shape of real spectral lines depends on the distribution of temperature and opacity. For low optical depth, the line has a Gaussian shape (if there is only one velocity component). The background intensity determines whether the line is in absorption $\left(I_{0}>S\left(T_{\mathrm{ex}}\right)\right)$ or in emission $\left(I_{0}<S\left(T_{\mathrm{ex}}\right)\right)$.

For high optical depth, one sees a black-body radiation with the excitation temperature at $\tau \approx 1$. At the maximum of $\Phi, \tau$ exceeds unity first, so regions closer to the observer are traced at the line center, while regions farther away are seen at frequencies more distant from $v_{u l}$. If the temperature is lower in the foreground region, self-absorption is observed: The center of the line is weaker than the edges, as the colder foreground molecules absorb the emission from the warmer background molecules.

In addition, $\Phi$ is shifted by the velocities along the line-of-sight. A high-velocity outflow produces broad wings, and an infall can lead to asymmetric self-absorption, with the blue side being stronger than the red side. The reason is that the foreground envelope, which recedes from us, can better absorb the emission from the hot inner gas which recedes as well, while the emission from the approaching gas can pass (Zhou \& Evans 1994). The asymmetry is stronger with larger infall velocities. Figure 2.3 illustrates this line shape.

\subsection{Observational Techniques}

After having described the origin of radiation, I will here briefly present a few aspects of the measurement of radio and submillimeter wavelengths. Details of radio telescopes can be found e.g. in Rohlfs \& Wilson (2004).

\subsubsection{Single-dish Telescopes}

A telescope collects the incoming parallel radiation by an antenna with a parabolic surface, and measures the intensity with a receiver. For instance, heterodyne receivers mix the signal with a tunable local oscillator and take spectra by measuring many frequencies simultaneously, or bolometers measure the temperature change due to the absorption of radiation. Through a secondary reflector, the focus can be adjusted to compensate for deformations of the telescope. 
The sensitivity depends on the collecting area, receiver noises, and atmospheric transmission. The noise from the whole system is summed up in the system temperature $T_{\text {sys. }}$. The observational noise (rms variation) for a frequency bandwidth $\Delta v$ and an integration time $t$ is

$$
\Delta T=\frac{T_{\text {sys }}}{\sqrt{\Delta v t}}
$$

The angular resolution is limited by diffraction. The beam pattern, which describes the angular dependence of the telescope's sensitivity, is the Fourier transform of the aperture. Thus, a point is spread to a beam of half power beam width (HPBW) $\theta=1.44 \frac{\lambda}{D}$ with wavelength $\lambda$ and telescope diameter $D$. Expressed in arcseconds,

$$
\theta=252^{\prime \prime} \frac{\lambda[\mathrm{mm}]}{D[\mathrm{~m}]}
$$

Scanning over a point source produces the beam shape, and is used to correct the pointing of the telescope when the position of the source is known. The sky is convolved by the beam, and the telescope can take either a single pointing (as an average over the sky weighted by the beam pattern) or a map that is smeared out with the beam. The beam consists of a main beam with approximately Gaussian shape of the above HPBW and sidelobes at larger angles. For a small source, only the main beam contributes, and the measured forward beam brightness temperature $T_{\mathrm{A}}^{*}$ has to be corrected for the main beam efficiency $B_{\text {eff }}$ (and re-corrected for the forward efficiency $\left.F_{\text {eff }}\right)$ to give the main beam brightness temperature

$$
T_{\mathrm{mb}}=\frac{F_{\mathrm{eff}}}{B_{\mathrm{eff}}} T_{\mathrm{A}}^{*}
$$

This is still less than the brightness temperature that a source smaller than the beam emits. For a hypothetical Gaussian source of FWHM $\sigma$, this beam dilution is given by the beam filling factor $\frac{\sigma^{2}}{\theta^{2}+\sigma^{2}}$.

To filter out the contribution of the atmosphere and the receiver bandpass, reference measurements are taken at positions off the source and subtracted from the measurements on the source. The data are calibrated by comparison with a source of known flux or a load of known temperature.

To convert units between brightness temperature (in K) and Jy/Beam $\left(1 \mathrm{Jy}=10^{-26} \frac{\mathrm{w}}{\mathrm{m}^{2} \mathrm{~Hz}}\right)$, one has to evaluate the solid angle of the beam $\Omega$. Assuming a circular beam with Gaussian shape $f(r)=e^{-4 \ln (2) \frac{r^{2}}{\sigma^{2}}}, \Omega=\int_{0}^{\infty} 2 \pi r f(r) d r=$ $\frac{\pi}{4 \ln (2)} \theta^{2}$ for $\theta$ in radian, i.e. 1/ln(2) larger than a circle with diameter $\theta$. Dividing the flux $F$ (in Jy) by $\Omega$ gives the flux density or intensity, which is converted to brightness temperature by applying the Rayleigh-Jeans approximation of the Planck function (equation 2.5):

$$
T_{\mathrm{b}}[\mathrm{K}]=13.6\left(\frac{\lambda[\mathrm{mm}]}{\theta\left[{ }^{\prime \prime}\right]}\right)^{2} F[\mathrm{Jy}]
$$

\subsubsection{Interferometry}

The low angular resolution of single-dish telescopes can be overcome by combining several antennas, thereby simulating a large telescope that consists mainly of gaps. The extension of the array (the largest baseline) can be used in equation (2.28) instead of the diameter to compute the angular resolution. The field of view is given by the beam size of a single antenna.

The signal received by each antenna is correlated with the other antennas, producing an interference pattern (fringes) that depends on the baseline and the source emission. The sky emission is Fourier-transformed by measuring interferences instead of the direct emission. The visibility, which is the interference contrast, is stored as amplitude and phase for each baseline and time interval, along with the orientation of the baseline relative to the source.

Angular coordinates are transformed to the Fourier space coordinates $u$ and $v$. The location of the antennas can be seen in this $u v$ plane, and a baseline represents a $u v$ point. While a single-dish telescope completely fills the $u v$ plane up to a maximum value given by its diameter, an interferometer has an incomplete $u v$ coverage, given by its array configuration relative to the source. Many baselines of different lengths are essential for a good $u v$ coverage. Tracking a source while the Earth rotates helps in sampling the $u v$ plane, as one baseline is stretched from a point to a part of an ellipse.

A point source would have the same amplitude on any baseline, but the extension of real sources causes a lower amplitude on the longer baselines. The missing of short baselines means filtering out of extended emission, which complicates the interpretation of interferometer data.

Atmospheric influence distorts phases and amplitudes. To correct for that, a point-like calibrator source has to be observed regularly between the target observations. The variations of its phase and amplitude are due to 
atmospheric changes. It should be close-by and strong. Before or after the track, a source of known flux has to be observed for the absolute flux calibration as well as a very strong source without line emission to correct for fluctuations in the bandpass (the spectral response of the receiver).

To restore the image, the calibrated visibilities have to be Fourier-transformed. The synthesized beam depends on the weighting of the visibilities. Natural weighting is assigning the same weight to each data point, uniform weighting gives the same weight to each $u v$ area, i.e. visibilities in sparsely sampled regions of the $u v$ plane have greater weights. Uniform weighting results in smaller beams, but larger noise. Different weighting schemes lying between these two are used, sometimes taking also the system temperature into account, thereby giving lower weights to measurements taken at low elevations, where the noise is increased due to the larger air mass.

Resulting beam and map are called 'dirty' as the beam has an irregular shape with positive and negative sidelobes due to missing spacings (the incomplete $u v$ coverage). The dirty map can be cleaned by finding maxima in the image (clean components), subtract their dirty beam, and add instead the clean components convolved with a clear (two-dimensional) Gaussian beam of the same HPBW. Still, the map might show systematic fluctuations which result from phase noise (the calibration is imperfect due to the low time resolution and the different locations of calibrator and source). Self-calibration is taking the clean components (or a known source structure) as representative of the real source and modifying the calibration (mostly of the phases) accordingly. After imaging again, the process can be repeated.

While a larger single-dish telescope has a smaller beam and therefore the same $\Delta T$ as a smaller one, the noise for an interferometer is approximately increased by the ratio of the area of the simulated telescope and the actual collecting area. Higher brightness sensitivity is thus reached with more compact configurations, giving lower resolution. For same resolution and collecting area, shorter wavelenghts increase the sensitivity, as the array can be more compact for the same beam. However, the system temperature usually increases with frequency because both receiver and atmosphere have higher contributions (see Fig. 1.4 for the atmospheric transmission).

\subsection{Modeling}

Analyzing the observations involves understanding how the measured radiation was produced. With the physical background provided in Sects. 2.1 to 2.4, it is possible to create models and compare their radiation to the observations. Deducing the source structure in a more direct way is impossible mainly due to the loss of the third spatial dimension that occurs in the integration of equation (2.16).

Section 2.6.1 describes how to compute the temperature, Sects. 2.6.2 and 2.6.3 present the radiative transfer codes RATRAN and RADMC-3D, respectively, which are used to compute the radiation that a model emits. In Sects. 2.6.4 to 2.6.6 I discuss how to define a frame for the source structure, how to compare model and data, and how to search the parameter space for good fits to the data.

\subsubsection{Temperature Computation}

A method to approximate the dust temperature in spherical symmetry with central heating is presented in Sect. 3.6.1.

The approximation of heating/cooling balance assumes that the radiation is an external field that is not influences by the dust. The dust grains are thus bathed in a fixed radiation field, and their temperature is determined by the interplay of heating by the radiation, which is independent of the dust temperature, and cooling by dust emission, which depends on $T^{4}$ and the dust opacity.

The diffusion approximation, in contrast, assumes that the dust is optically thick to its own radiation, so it cannot cool easily. Instead, dust emission is constantly re-absorbed, and the radiation diffuses outwards. This radiative trapping leads to a steeper temperature gradient and higher temperatures in optically thick regions that harbor an internal heating source. The efficieny of diffusion depends on geometry, as the radiation can better escape in the case of an inhomogeneous medium, e.g. a disk or filaments.

Although the above methods are good approximations in their regimes, an exact way of computation has to be iterative. The dust temperature depends on the radiation field, which in turn is, to varying degrees, influenced by the dust temperature through the cooling radiation.

Dust radiative transfer codes explicitly perform these computations, e.g. DUSTY (Ivezic et al. 1999) or RADMC3D (Sect. 2.6.3). They are usually slower with larger optical depth, since the photons are absorbed and emitted more often.

As for the level populations, the timescales are short enough that one can assume an equilibrium. An exact determination of the temperature would have to take into account the different cooling and heating of dust and gas and the coupling of their temperatures, which is stronger at high densities. 


\subsubsection{RATRAN}

The radiative transfer code RATRAN, developed by Hogerheijde \& van der Tak (2000), calculates the excitation and radiative transfer of molecular lines, using an accelerated Monte Carlo method for the determination of level populations. The manual can be found at http://www.sron.rug.nl/ṽdtak/ratran. The code consists of two parts. AMC computes the level populations for a given source structure, SKY does the ray-tracing to create maps in a certain transition.

AMC involves two steps. A fixed set of random rays is propagated through the source, iterating the level populations until a certain accuracy is reached. In the second step, this is repeated for varying ray directions. The number of photons is doubled for each new set, until the level populations and the radiation are consistent. The procedure is accelerated for large optical depths by treating local contributions to the radiation field separately.

SKY solves the equation (2.19) along the line-of-sight for each cell consecutively starting from the back. This is repeated for each pixel and channel. Frequency-dependent opacity and source function are known from the level populations, the temperature, and the dust opacity. Only one line can be computed at once, so line overlapping is not taken into account.

Inputs are a molecular data file, a description of the physical and chemical structure of the model, and instructions for AMC and SKY. The molecular data file can be taken from the LAMDA ${ }^{3}$ database, containing energy of the levels, their statistical weights, the Einstein A coefficients of the transitions and the collisional rate coefficients for different temperatures. The model is usually spherically symmetric and consists of shells. Two-dimensional models are also possible in a special version of the code. Each shell has specified inner and outer radius, density of $\mathrm{H}_{2}$ and of the molecule, temperature of dust and gas, line width and radial velocity. In addition to numerical parameters, dust opacity and distance are specified in the remaining input files.

\subsubsection{RADMC-3D}

The three-dimensional dust and line radiative transfer code RADMC-3D, developed by C. Dullemond, computes the dust temperature and the resulting continuum and line radiation. A detailed manual can be found at http://www.ita.uni-heidelberg.de/dullemond/software/radmc-3d.

The dust temperature is computed by tracing photon packets which are randomly emitted by the stars. They represent a certain spectrum and are absorbed by the dust, which is thereby heated and emits its own photon packet, which is traced through the model until it escapes through the boundaries. With growing number of emitted photon packets, the dust temperature becomes more accurate.

The ray-tracing uses recursive sub-pixeling to accurately account for structures smaller than a pixel. Maps at any desired frequency can be made; for lines these frequencies sample the line shape. The opacity of a cell is the sum of the opacities of dust, ionized gas, and all lines that are included. Line overlapping is thus taken into account.

Level populations are determined assuming LTE. As additional methods, the LVG method is already implemented (Shetty et al. 2011), and full non-LTE line transfer is planned.

Inputs include dust opacity, molecular data, and location and properties of stars. A flexible module that the user may edit (in FORTRAN90) allows adaptive mesh refinement and the definition of parameters, e.g. for the density.

\subsubsection{Defining Parameters}

Setting up a model begins with a fundamental decision. There are infinite possibilities of structures that a source could have, and a model is necessarily a simplification and has to follow assumptions and fundamental limits. For instance, a model is assumed to be homogeneous (maybe with different, independent components), to follow geometrical symmetries, or to have statistical distributions, described by certain parameters. The parameter set implicitly pre-defines the structure of a model, and this structure is the only one that can be tested by varying parameters.

Model structures can be based on (magneto-)hydrodynamic simulations, or follow some kind of analytical shape. In either case, the structure is parameterized to allow adjustment to the data. An example of a complicated model, which is described by more parameters, is an independent density in each cell, requiring one parameter for each cell. Such more complicated models have higher chances to reproduce the data, but the large number of parameters makes it very difficult to find this parameter combination and to interpret it. Therefore, the number of parameters should be kept small (usually on the expense of tighter limitations, though).

Physical relations can help to reduce the number of parameters. The distribution of density, temperature, abundances, and velocity depend on each other, although in a complex way which might not be possible to compute.

\footnotetext{
${ }^{3}$ http://www.strw.leidenuniv.nl/ monoldata
} 
An example is the temperature, which can be determined from heating and the density distribution. In this case, the temperature shape is not described by parameters, but implicitly by the luminosity of stars and the density shape.

\subsubsection{Comparison of Model and Data}

When the radiative transfer code has produced a map with arbitrary spectral and spatial resolution (the specified channel width and pixel size), this map has to be 'observed' by a telescope to compare it to the observational data. For a single-dish telescope, this means convolving the map with the telescope beam, usually approximated as a Gaussian with the width given by equation (2.28). The spectrum is then extracted from where the telescope pointed. To account for the $u v$ coverage of an interferometer, the map has to be Fourier-transformed and the same imaging procedure performed as for the data. The resulting map is of course free from the noise which distorts the data map. To avoid the additional uncertainties involved in the imaging procedure, model and data could also be compared directly in $u v$ space.

A simple way of comparing model and data is to overlay the spectra and/or display the maps next to each other. One can then see by eye which aspects of the model fit satisfactorily to the data and which need adjustment. However, this judgment is subjective.

A quantitative estimate of how well a model reproduces the data can be made by computing the $\chi^{2}$, i.e. the average of the squares of the difference between model and data points. While that can be computed relatively easy, each data point must be associated with a weighting factor that determines its relative weight in the summation over all data points. Weighting can be done by thermal noise, but this is often not the desired weighting scheme. In particular, some lines are considered more important, and not all spectral and spatial regions constrain the model equally well; instead the line cores and the source center could be given a larger weight. This weighting substantially influences the evaluation of a model, and has to be chosen carefully.

Running a model and comparing its output to the data can be made with flexible scripts that I wrote in PYTHON. Such scripts convert the given set of parameters into the input needed by the radiative transfer code, run the program, and process the output. $\chi^{2}$ can be computed, and several parameter sets can be tested at once.

\subsubsection{Searching the Parameter Space}

A straightforward way to search for a suitable model is the manual adjustment of parameters. After evaluation of a model by comparison to the data, the parameters are varied and the new model is computed and evaluated. Obviously, the art is to decide how to vary the parameters in order to improve the fit. Due to the very complex dependences of observational quantities on the parameters, this is a difficult task requiring a lot of trials and errors.

A brute-force method is to sample the parameter space as completely as possible with the given computational limits. Each parameter is divided into a number of values, spanning the region of interest. Each combination of the values of the different parameters is computed, and the models are evaluated by their $\chi^{2}$. This allows to find the best-fitting model, as well as an error estimate of its parameters, in this grid. The major drawback of the complete computation of the discretized parameter space is the huge computational effort - for $N$ parameters with $M$ values each, $M^{N}$ models have to be computed.

The third method is to employ algorithms which search the parameter space. These algorithms choose the new parameters on the basis of previous variations of $\chi^{2}$ with changing parameters. Examples are the LevenbergMarquardt algorithm, which moves down the $\chi^{2}$ gradient and thereby finds the local minimum of $\chi^{2}$. Algorithms based on swarm intelligence also test parameter combinations that are far away from local minima, but concentrate on regions of low $\chi^{2}$. The parameters can be constrained and error estimates provided. Although more intelligent than the grid method, it too has to compute many models, which is a problem if a model run needs a large computational effort. A flexible fitting engine that makes use of various algorithms, MAGIX, is currently under development ${ }^{4}$.

Sometimes a close relationship between an observational quantity and a parameter can be found. In the case of a monotonous dependence (the larger the parameter, the larger the quantity), the parameter may be automatically adjusted by using the ratio of the model and data quantity. For instance, the intensity of an optically thin line is related to the molecular abundance in this way. The abundance can be adjusted by an automatic variation based on the ratio of modeled and observed intensity.

Continuum and lines can often both be computed separately and be separated in the observations. As the computation for the lines is usually much more elaborate than for the continuum, the relative independence of the continuum from the lines offers the possibility to first compute the continuum, compare it to the observations, and compute the lines only if the fit is satisfactory. This method substantially limits the parameter space that has to be searched, as only the continuum-relevant parameters have to be searched completely, while the search of the full parameter set is operated on a subset of these parameters.

\footnotetext{
${ }^{4}$ http://www.astro.uni-koeln.de/projects/schilke/MAGIX
} 


\section{Chapter 3}

\section{APEX paper: Structure of evolved cluster-forming regions}

Sections 3.2 to 3.10 appeared in Astronomy $\mathcal{E}$ Astrophysics: Rolffs, R., Schilke, P., Wyrowski, F., Menten, K. M.,

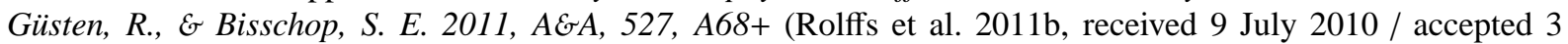
December 2010)

\subsection{Overview}

Telescope: APEX (Atacama Pathfinder EXperiment) is a 12-m telescope in the Atacama desert in Northern Chile at an altitude of $5100 \mathrm{~m}$. The instruments include heterodyne spectrometers covering 211-370 GHz, a bolometer array for continuum maps at $345 \mathrm{GHz}$ (LABOCA, the Large Apex BOlometer CAmera), and CHAMP+, a 7pixel spectral line receiver covering 602-720 and 790-950 GHz. APEX is operated by the Max-Planck-Institut für Radioastronomie (MPIfR), the European Southern Observatory (ESO) and the Onsala Space Observatory.

Observations: HCN lines (4-3 and 9-8) toward eight hot core sources were observed in 2006 by Peter Schilke and Claudia Comito, which formed the basic data set of my diploma thesis (Rolffs 2007). In 2008, I wrote a successful observing proposal for far more lines (from $\mathrm{HCN}, \mathrm{HCO}^{+}$, and $\mathrm{CO}$ ) in 12 sources, using the APEX-1, APEX-2, and CHAMP+ receivers.

Modeling: To compute the temperature in a centrally heated sphere, I used an approximate method: diffusion inside the photosphere and heating/cooling balance outside. It was necessary to develop this method because available dust radiative transfer codes (such as DUSTY, Ivezic et al. 1999) could not compute models of very high optical depths. From a large grid of parameters, models were selected that fit the radial profile of the $345 \mathrm{GHz}$ continuum (extracted from LABOCA data). In an additional grid, abundance structures were tested by automatically adapting the abundance values to fit optically thin lines, computed by RATRAN. Finally, the abundances were manually fine-tuned to produce one model for each source.

Results: Many lines, being very optically thick, display complicated line shapes with self-absorption, asymmetries, and outflow wings. The most striking observational result is the high detection rate of strong vibrationally excited $\mathrm{HCN}$ and, in half of the sources, even $\mathrm{H}^{13} \mathrm{CN}$. This suggests that the amount of hot molecular gas is much higher than previously thought. The lines from the vibrational state could be well modeled; in those sources with detected $\mathrm{H}^{13} \mathrm{CN}$ the $\mathrm{HCN}$ abundance has to increase with temperature. The blue asymmetric self-absorbed profiles could also be modeled, resulting from temperature gradients and an infalling velocity field, often requiring a lower $\mathrm{HCN}$ and $\mathrm{HCO}^{+}$abundance in the outer region. However, the situation is not that simple in many sources, where also red asymmetries and irregular shapes occur. Moreover, emission from high- $J$ lines in the outer pixels of the $\mathrm{CHAMP}+$ receiver could not be reproduced by the models. This must be due to clumpiness and multiplicity of heating. Also, the lack of self-absorption in two sources is caused by such geometry effects.

\subsection{Abstract}

Context: An approach towards understanding the formation of massive stars and star clusters is to study the structure of their hot core phase, an evolutionary stage where dust has been heated, but molecules have not yet 
Table 3.1: Sources observed with the APEX telescope. Peak flux and full width at half-maximum are extracted from ATLASGAL $(850 \mu \mathrm{m})$.

\begin{tabular}{|c|c|c|c|c|c|c|c|c|}
\hline Source & Abbreviation & $\begin{array}{c}\text { Distance } \\
{[\mathrm{kpc}]}\end{array}$ & $\begin{array}{c}\text { Luminosity }^{a} \\
{\left[\mathrm{~L}_{\odot}\right]}\end{array}$ & $\begin{array}{l}\text { Peak Flux } \\
\text { [Jy/Beam] }\end{array}$ & $\begin{array}{c}\text { FWHM } \\
{\left[{ }^{\prime \prime}\right]}\end{array}$ & $\begin{array}{c}\text { RA } \\
(\mathrm{J} 2000)\end{array}$ & $\begin{array}{c}\text { Dec } \\
(\mathrm{J} 2000)\end{array}$ & $\begin{array}{c}\text { LSR Velocity } \\
{[\mathrm{km} / \mathrm{s}]}\end{array}$ \\
\hline IRAS 12326-6245 & $\mathrm{i} 12$ & $4.4^{1}$ & $2.7(5)^{2}$ & 22 & 26.5 & $12: 35: 35.06$ & $-63: 02: 31.0$ & -39.3 \\
\hline G327.3-0.6 & g327 & $2.9^{3}$ & $1(5)^{4}$ & 48 & 27.5 & $15: 53: 07.8$ & $-54: 37: 06.4$ & -44.0 \\
\hline IRAS 16065-5158 & i16 & $4^{2}$ & $2.9(5)^{2}$ & 17 & 27.8 & $16: 10: 20.0$ & $-52: 06: 08.8$ & -62.2 \\
\hline NGC6334(I) & ngc & $1.7^{5}$ & $2.6(5)^{6}$ & 56 & 28.6 & $17: 20: 53.44$ & $-35: 46: 57.9$ & -8.0 \\
\hline IRAS 17233-3606 & i17 & $1^{7}$ & $2.7(4)^{2}$ & 49 & 27.0 & $17: 26: 42.5$ & $-36: 09: 18.1$ & -3.4 \\
\hline $\operatorname{SgrB} 2(N)$ & $\mathrm{b} 2 \mathrm{n}$ & $7.8^{8}$ & $8.4(5)^{9}$ & 147 & 27.8 & $17: 47: 19.88$ & $-28: 22: 18.4$ & +64.0 \\
\hline $\operatorname{SgrB2}(\mathrm{M})$ & $\mathrm{b} 2 \mathrm{~m}$ & $7.8^{8}$ & $6.3(6)^{9}$ & 126 & 32.6 & $17: 47: 20.17$ & $-8: 23: 04.6$ & +60.0 \\
\hline $\mathrm{G} 10.47+0.03$ & g10 & $10.6^{10}$ & $7(5)^{11}$ & 26 & 25.3 & $18: 08: 38.24$ & $--19: 51: 50.2$ & +68.0 \\
\hline $\mathrm{G} 31.41+0.31$ & g31 & $7.9^{12}$ & $2.6(5)^{13}$ & 22 & 26.7 & $18: 47: 34.31$ & $-01: 12: 45.9$ & +97.0 \\
\hline G34.26+0.15 & g34 & $3.7^{14}$ & $4.7(5)^{12}$ & 51 & 29.1 & $18: 53: 18.57$ & $+01: 14: 58.3$ & +58.0 \\
\hline W51d & w51d & $5.4^{15}$ & $2.4(6)^{16}$ & 39 & 30.0 & $19: 23: 39.9$ & $+14: 31: 10.1$ & +60.0 \\
\hline W51e & w51e & $5.4^{15}$ & $1.2(6)^{16}$ & 74 & 29.1 & $19: 23: 43.91$ & $+14: 30: 28.4$ & +56.0 \\
\hline
\end{tabular}

References. (1) Osterloh et al. (1997); (2) Faúndez et al. (2004); (3) Simpson \& Rubin (1990); (4) Wyrowski et al. (2006); (5) Neckel (1978); (6) Sandell (2000); (7) MacLeod et al. (1998); (8) Reid et al. (2009); (9) Goldsmith et al. (1992); (10) Pandian et al. (2008); (11) Cesaroni et al. (2010); (12) Churchwell et al. (1990); (13) Cesaroni et al. (1994); (14) Watt \& Mundy (1999); (15) Sato et al. (2010); (16) Rudolph et al. (1990).

Notes. ${ }^{(a)}$ The numbers in parentheses are powers of 10.

been destroyed by ultraviolet radiation. These hot molecular cores are very line-rich, but the interpretation of line surveys is also hampered by poor knowledge of the physical and chemical structure.

Aims: To constrain the radial structure of high-mass star-forming regions containing hot cores, we attempt to reproduce by radiative transfer modeling both the intensity and shape of a variety of molecular lines.

Methods: We observed 12 hot cores with the Atacama Pathfinder Experiment (APEX) in lines of $\mathrm{HCN}^{\mathrm{HCO}} \mathrm{HCO}^{+}$ $\mathrm{CO}$, and their isotopologues, including high-J lines and vibrationally excited HCN. We investigate how well the sources can be modeled as centrally heated spheres with a power-law density gradient, making use of the radiative transfer code RATRAN and the radial profile of the submm continuum emission, taken from the APEX Telescope Large Area Survey of the GALaxy (ATLASGAL).

Results: Most of the observed lines have complicated shapes that incorporate self-absorption, asymmetries, and line wings. Vibrationally excited $\mathrm{HCN}$ is detected in all sources, and vibrationally excited $\mathrm{H}^{13} \mathrm{CN}$ in half of the sources. We are able to successfully model most features seen in the APEX data, such as the ratio of the isotopologue lines (very high optical depths), self-absorption (temperature gradient), blue asymmetries (moderate infall), vibrationally excited $\mathrm{HCN}$ (high inner temperatures), and $\mathrm{H}^{13} \mathrm{CN}$ (high $\mathrm{HCN}$ abundance under dense and hot conditions). Other features could not be reproduced, such as an occasional lack of self-absorption, the emission from high-J lines in the outer pixels of the CHAMP+ receiver $\left(15^{\prime \prime}-20^{\prime \prime}\right.$ from the center), the outflow wings, and the red asymmetric profiles.

Conclusions: The amount of molecular gas, in particular of $\mathrm{HCN}$, at very high temperatures is larger than previously thought. A complex interplay between infall and outflow motions is present. Our basic model assumptions of pure central heating and a power-law radial density distribution can serve as approximations for most sources, but are too simple to explain all observed lines. In particular, taking into account clumpiness, multiplicity of heating sources and a more complex velocity field seems to be necessary to more closely match model calculations to observations. This would require three-dimensional radiative transfer modeling of high-resolution interferometric data.

\subsection{Introduction}

Hot molecular cores are early stages of massive star formation, where stellar feedback is starting to affect the remnant core. The dense molecular gas out of which massive stars form is heated by their high luminosity, and shocks impinge on it, but it is not yet destroyed by ionization. While radiation from hypercompact HII regions 
Table 3.2: Frequency Settings.

\begin{tabular}{|c|c|c|c|}
\hline Receiver & Frequency Coverage [GHz] & Lines & Sources \\
\hline APEX-2A & $341.5-343.1 \& 353.5-355.1$ & HCN 4-3 & g327, ngc, b2n, b2m, g10, g31, w51d, w51e \\
\hline APEX-2A & $343.9-345.6 \& 355.9-357.6$ & $\mathrm{H}^{13} \mathrm{CN} \& \mathrm{HC}^{15} \mathrm{~N} 4-3$ & g327, ngc, i17, b2n, b2m, g10, g31, w51d, w51e \\
\hline FLASH & $460.2-461.9 \& 466.2-467.9$ & $\mathrm{CO} 4-3$ & g327, ngc, g10, g31 \\
\hline FLASH & 796.9-798.6 \& 808.9-810.6 & $\mathrm{HCN} 9-8$ & g327, ngc, b2n, b2m, g10, g31, w51d, w51e \\
\hline APEX-1 & $224.2-225.2 \& 226.6-227.6$ & $\mathrm{C}^{17} \mathrm{O} 2-1$ & $\mathrm{i} 12, \mathrm{i} 16$ \\
\hline APEX-1 & $257.7-259.5$ & $\mathrm{H}^{13} \mathrm{CN} \& \mathrm{HC}^{15} \mathrm{~N} 3-2$ & i12, i16, ngc, b2n, b2m, g10, g34, w51d, w51e \\
\hline APEX-1 & $259.8-261.6$ & $\mathrm{H}^{13} \mathrm{CO}^{+} \& \mathrm{HC}^{17} \mathrm{O}^{+} 3-2$ & i12, i16, b2n, b2m, g10, g34, w51d, w51e \\
\hline APEX-1 & $265.4-266.4 \& 267-268$ & $\mathrm{HCN} \& \mathrm{HCO}^{+} 3-2$ & i12, i16, ngc, b2n, g10, g31, g34, w51d, w51e \\
\hline APEX-2 & $340.4-341.4 \& 343.4-344.4$ & $\mathrm{HC}^{18} \mathrm{O}^{+} \& \mathrm{HC}^{15} \mathrm{~N} 4-3$ & $\mathrm{i} 12, \mathrm{~g} 327, \mathrm{i} 16, \mathrm{ngc}, \mathrm{b} 2 \mathrm{n}, \mathrm{b} 2 \mathrm{~m}, \mathrm{~g} 10, \mathrm{~g} 31, \mathrm{w} 51 \mathrm{~d}, \mathrm{w} 51 \mathrm{e}$ \\
\hline APEX-2 & $345-346 \& 346.6-347.6$ & $\mathrm{H}^{13} \mathrm{CN} \& \mathrm{H}^{13} \mathrm{CO}^{+} 4-3 \& \mathrm{CO} 3-2$ & i12, g327, i16, ngc, b2n, b2m, g10, g31, g34, w51d, w51e \\
\hline APEX-2 & $354-355 \& 356-357$ & $\mathrm{HCN} \& \mathrm{HCO}^{+} 4-3$ & $\mathrm{i} 12, \mathrm{~g} 327, \mathrm{i} 16, \mathrm{ngc}, \mathrm{b} 2 \mathrm{~m}, \mathrm{~g} 10, \mathrm{~g} 34, \mathrm{w} 51 \mathrm{~d}, \mathrm{w} 51 \mathrm{e}$ \\
\hline $\mathrm{CHAMP}^{+}$ & $657.9-660.5$ & $\mathrm{C}^{18} \mathrm{O} 6-5$ & i12, g327, i16, ngc, i17, b2n, b2m, g10, g34, w51d, w51e \\
\hline $\mathrm{CHAMP}^{+}$ & $689.6-692.3$ & $\mathrm{H}^{13} \mathrm{CN} 8-7 \& \mathrm{CO} 6-5$ & i12, g327, i16, ngc, i17, b2n, b2m, g10, g34, w51d, w51e \\
\hline $\mathrm{CHAMP}^{+}$ & $693.5-696$ & $\mathrm{H}^{13} \mathrm{CO}^{+} 8-7$ & g327, ngc, i17, b2n, b2m, g10, g34, w51d, w51e \\
\hline $\mathrm{CHAMP}^{+}$ & 708.1-710.7 & $\mathrm{HCN} 8-7$ & i12, g327, i16, ngc, i17, b2n, b2m, g10, g34, w51d, w51e \\
\hline $\mathrm{CHAMP}^{+}$ & $800-802.7$ & $\mathrm{HCN}, \mathrm{v}_{2}=19-8$ & g327, ngc, g10, w51e \\
\hline $\mathrm{CHAMP}^{+}$ & $876-878.6$ & $\mathrm{C}^{18} \mathrm{O} 8-7$ & $\mathrm{i} 12, \mathrm{~g} 327, \mathrm{i} 16, \mathrm{ngc}, \mathrm{i} 17, \mathrm{~b} 2 \mathrm{n}, \mathrm{b} 2 \mathrm{~m}, \mathrm{~g} 10, \mathrm{~g} 34, \mathrm{w} 51 \mathrm{~d}, \mathrm{w} 51 \mathrm{e}$ \\
\hline $\mathrm{CHAMP}^{+}$ & $879.4-882$ & ${ }^{13} \mathrm{CO} 8-7$ & i12, g327, i16, ngc, i17, b2n, b2m, g10, g34, w51d, w51e \\
\hline $\mathrm{CHAMP}^{+}$ & $889.4-892$ & $\mathrm{HCO}^{+} 10-9$ & i12, g327, i16, ngc, i17, b2n, b2m, g10, g34, w51d, w51e \\
\hline
\end{tabular}

Notes. APEX-2A and FLASH are double-sideband receivers (observations in 2006). APEX-1, APEX-2, and CHAMP ${ }^{+}$are single-sideband receivers (observations in 2008). In addition to the lines listed here, every HCN line has a vibrational satellite at around $40 \mathrm{~km} / \mathrm{s}$ lower in frequency. The other vibrational satellite line is a few $\mathrm{GHz}$ higher in frequency.

is observable in some cases, ionization is still kept too compact by the high densities caused, e.g., by ongoing accretion, to affect significant volumes (Walmsley 1995). Ice mantles around dust grains evaporate or are removed by shocks. Their complex chemistry and high temperatures make hot cores very line-rich and prominent targets for line surveys (e.g. Schilke et al. 2006; Belloche et al. 2007; Bergin et al. 2010). Owing to the large mass of warm dust, they are the brightest interstellar sources in the submm sky. Since high-mass stars quickly ionize their surroundings and drive out the gas, hot cores must be short-lived objects. They are indeed destroyed by the expanding ultracompact HII regions ionized by their central star.

Despite their importance to both astrochemistry and massive star formation, little is known about the internal structure of hot cores and their envelopes (the distribution of density, temperature, molecular abundances, and velocity field). Reasons for this are difficulties in observations and in data analysis. These objects are compact $(\approx 0.1 \mathrm{pc})$, rare (a few dozens are currently known in the Galaxy), and hence typically at large distances. The high dust column densities make them invisible in the near infrared and at shorter wavelengths. Single-dish radio and submm telescopes do not have high enough angular resolution to resolve the sources, and the still limited interferometer data available today are confusing and hard to interpret. Hot cores are found at the formation sites of clusters, so their structure is not simple and the interpretation of observations is not straightforward, but has to rely on radiative transfer modeling, i.e. a comparison between model and data.

Several attempts have been made to model the structure of hot cores in spherical symmetry, often based on their spectral energy distribution (SED) (van der Tak 2002). For instance, Hatchell \& van der Tak (2003) modeled the continuum and CS integrated line intensity, Osorio et al. (2009) reproduced the $\mathrm{NH}_{3}(4,4)$ line in $\mathrm{G} 31.41+0.31$ (VLA, Cesaroni et al. 1998), or Nomura \& Millar (2004) coupled physical and chemical models to predict abundances in Orion. However, no multi-line study trying to reproduce line shapes has yet been attempted. In this paper, we present APEX observations of many $\mathrm{HCN}, \mathrm{HCO}^{+}$, and $\mathrm{CO}$ lines in 12 hot cores, as well as a comparison with models.

The models are spherically symmetric and have power-law density gradients. The temperature is determined self-consistently from central heating (Sect. 3.6.1). Continuum and line emission and absorption are computed using the code RATRAN (Hogerheijde \& van der Tak 2000, Sect. 3.7.1). To avoid searching blindly in the large, multi-dimensional parameter space, we develop a method that approaches a possible good fit. From a grid of parameters, we select the models that fit the radial profile of the $850 \mu \mathrm{m}$ continuum (Sect. 3.6), and identify suitable abundances (Sect. 3.7). 
Table 3.3: Line ratios.

\begin{tabular}{lcccccccccccc}
\hline \hline Line ratio & i12 & g327 & i16 & ngc & i17 & b2n & b2m & g10 & g31 & g34 & w51d & w51e \\
\hline $\mathrm{HCN}, v_{2}=1 / \mathrm{HC}^{15} \mathrm{~N}, v=0(4-3)$ & 0.25 & 0.7 & 0.6 & 0.5 & 0.8 & 1 & 1.5 & 1 & 0.3 & $0.25^{a}$ & 0.9 & 0.3 \\
$\mathrm{H}^{13} \mathrm{CN}, v_{2}=1 / \mathrm{HC}^{15} \mathrm{~N}, v=0(4-3)$ & $<0.1$ & 0.3 & 0.1 & $<0.15$ & 0.08 & 0.5 & 0.7 & 0.7 & $<0.15$ & $<0.1^{a}$ & $<0.2$ & $<0.15$ \\
\hline $\mathrm{H}^{13} \mathrm{CN}, v=0 / \mathrm{HC}^{15} \mathrm{~N}, v=0(4-3)$ & 4 & 1.5 & 2.5 & 2.5 & 2 & 0.8 & 4.5 & 3.5 & 3 & $2.5^{a}$ & 3.5 & 2.8 \\
$\mathrm{H}^{13} \mathrm{CO}^{+} / \mathrm{HC}^{18} \mathrm{O}^{+}(4-3)$ & 10 & 4 & 8 & 5 & $\ldots$ & 2 & 4 & $>2$ & 3.5 & $\ldots$ & $>5$ & 3 \\
${ }^{13} \mathrm{CO} / \mathrm{C}^{18} \mathrm{O}(8-7)$ & 2.5 & 2 & 2.3 & 3.3 & 1.8 & 2 & 1.3 & 1.3 & $\ldots$ & 2.5 & 4.4 & 2 \\
\hline
\end{tabular}

Notes. ${ }^{(a)} 3-2$ transition

\subsection{Observations and data reduction}

APEX (Atacama Pathfinder Experiment) ${ }^{1}$ is a $12-\mathrm{m}$ telescope located at $5100 \mathrm{~m}$ altitude in the northern Atacama desert in Chile (Güsten et al. 2006). This paper is based entirely on observations performed with this telescope. The sources observed are listed in Table 3.1. All data were taken in position switching mode, with the off positions $100^{\prime \prime}$ (wobbler for CHAMP+) or 400" (other settings) in azimuth from the source center. Beam sizes vary between $24^{\prime \prime}$ at $260 \mathrm{GHz}, 18^{\prime \prime}$ at $345 \mathrm{GHz}, 9^{\prime \prime}$ at $690 \mathrm{GHz}$, and 7" at $890 \mathrm{GHz}$. Pointing corrections were on the order of $5^{\prime \prime}$.

The first part of the data were taken in 2006 for eight sources. For the HCN 9-8 line, the FLASH receiver (Heyminck et al. 2006) and for the 4-3 lines, the APEX-2A receiver (Risacher et al. 2006) was used. Since these are double-sideband receivers, three settings with slightly different band center frequencies (spaced by $80 \mathrm{MHz}$ ) were taken to identify blending from the other sideband. In 2008, we observed lines from different isotopologues of $\mathrm{HCN}, \mathrm{HCO}^{+}$, and CO with the APEX-1, APEX-2 (Vassilev et al. 2008), and CHAMP ${ }^{+}$receivers (Kasemann et al. 2006; Güsten et al. 2008), all of which are single-sideband receivers. CHAMP $^{+}$allows simultaneous observations in the $690 \mathrm{GHz}$ and the $850 \mathrm{GHz}$ bands. Although it is a seven-pixel array receiver, we did not compile maps because the sources are very compact; we thus chose a higher signal-to-noise ratio $(\mathrm{S} / \mathrm{N})$ in the central pixel, and used the average of the outer pixels as an effective offset position ( 2.15 beam sizes, $15^{\prime \prime}-20^{\prime \prime}$, from the center). The fast Fourier transform spectrometer (Klein et al. 2006) was used as the backend for all observations. Table 3.2 summarizes the frequency settings. In addition, $\mathrm{CO} 4-3$ and 7-6 observations of nine sources performed by Friedrich Wyrowski, as well as observation of HCN lines from the line survey of G327.3-0.6 by Suzanne Bisschop were used to supplement our data set.

The data reduction involved the following steps. Using CLASS from the GILDAS software package ${ }^{2}$, the individual scans were summed, rejecting obviously bad ones. To convert from corrected antenna temperature $\mathrm{T}_{A}^{*}$ to main-beam brightness temperature $\mathrm{T}_{m b}$, the intensity was multiplied by the forward efficiency of 0.95 and divided by the main beam efficiency, which is 0.73 below $400 \mathrm{GHz}$. For the FLASH receiver, it is 0.6 below 600 $\mathrm{GHz}$ and 0.43 above (Güsten et al. 2006). For $\mathrm{CHAMP}^{+}$in July 2008, we adopted 0.28 below $750 \mathrm{GHz}$ and 0.3 above. For the September 2008 data, we used 0.38 below $750 \mathrm{GHz}$ and 0.35 above $^{3}$

A polynomial of order between 0 and 5 was fitted to the channels less affected by lines and used as a baseline that was subtracted from the spectra. The lines from $\mathrm{HCN}, \mathrm{HCO}^{+}$, and $\mathrm{CO}$ were extracted, and, if necessary, another baseline subtraction was done. In some spectra, baseline ripples of period not much longer than the line width hampered this procedure. Finally, if lines were observed in different frequency settings, they were averaged to improve the $\mathrm{S} / \mathrm{N}$.

The intensity error can be estimated from varying intensities in these different settings. While it was usually within $30 \%$, sometimes a factor of 2 or even 3 could be seen. Pointing was usually done on the source itself, but the drifting of the pointing and focus is a major problem for the high-frequency observations. The intensities of the $\mathrm{HCN}$ isotopologue lines are typically 30-50\% larger in the APEX-2A data than in the APEX-2 data, sometimes twice as large. We chose to trust the newer APEX-2 observations, since for G327.3-0.6 they are consistent with Suzanne Bisschop's data, taken with the same receiver. In addition, the APEX-2A receiver is known to have problems with varying sideband gain ratios affecting the calibration.

Radial $850 \mu \mathrm{m}$ intensity profiles for all sources were extracted from ATLASGAL (APEX Telescope Large Area Survey of the Galaxy, Schuller et al. 2009), which uses LABOCA (Large APEX Bolometer Camera) on APEX (Siringo et al. 2009). The peak of the dust emission was taken as the center of the radial profiles. Regridding to $1^{\prime \prime}$ pixel size and radial averaging was done with MIRIAD ${ }^{4}$ (Sault et al. 1995).

\footnotetext{
${ }^{1}$ This publication is based on data acquired with the Atacama Pathfinder Experiment (APEX). APEX is a collaboration between the MaxPlanck-Institut fur Radioastronomie, the European Southern Observatory, and the Onsala Space Observatory.

${ }^{2}$ http://www.iram.fr/IRAMFR/GILDAS

${ }^{3}$ http://www.mpifr.de/div/submmtech

${ }^{4} \mathrm{http}: / /$ bima.astro.umd.edu/miriad
} 
Table 3.4: Integrated line intensities (in $\mathrm{K} \mathrm{km} / \mathrm{s}$ ).

\begin{tabular}{lcccccccccccc}
\hline \hline Line & $\mathrm{i} 12$ & $\mathrm{~g} 327$ & $\mathrm{i} 16$ & $\mathrm{ngc}$ & $\mathrm{i} 17$ & $\mathrm{~b} 2 \mathrm{n}$ & $\mathrm{b} 2 \mathrm{~m}$ & $\mathrm{~g} 10$ & $\mathrm{~g} 31$ & $\mathrm{~g} 34$ & w51d & w51e \\
\hline $\mathrm{HC}^{15} \mathrm{~N} \mathrm{4-3}$ & 6.2 & 14.4 & 8.9 & 9.2 & 45.2 & 57.8 & 31.6 & 3.1 & 6.3 & $15^{a}$ & 6.1 & 15.6 \\
$\mathrm{HC}^{18} \mathrm{O}^{+} 4-3$ & 1.7 & 6.9 & 2.2 & 1.1 & $\ldots$ & 92.9 & 39.2 & 3.5 & 2.2 & $\ldots$ & low & 9.1 \\
$\mathrm{C}^{18} \mathrm{O} 6-5$ & 171.2 & 122.8 & 155.4 & 114.7 & 255.9 & 414.6 & 844.6 & 87.1 & $\ldots$ & 174.2 & 99.1 & 237.5 \\
$\mathrm{C}^{18} \mathrm{O} 8-7$ & 60.1 & 50.4 & 50.5 & 47.8 & 85.6 & 192.1 & 502.1 & 57.7 & $\ldots$ & 81.7 & 65.8 & 129.9 \\
$\mathrm{HCN}, v_{2}=13-2$ & 1.9 & 9.2 & 5.7 & 3.1 & $41.6^{b}$ & 63.2 & 43.8 & 4.8 & 1.3 & 2.9 & 2.6 & 8.0 \\
$\mathrm{H}^{13} \mathrm{CN}, v_{2}=18-7$ & low & 15.6 & 3.4 & low & 11.0 & 42.8 & 42.4 & 15.0 & $\ldots$ & low & low & low \\
\hline $\mathrm{SiO} 8-7$ & 25.8 & 9.1 & 3.8 & 3.7 & $\ldots$ & 60.0 & 63.6 & 8.2 & 12.7 & 28.6 & 21.3 & 14.4 \\
$\mathrm{HC}$ & & 12.1 & 11.8 & 5.3 & 49.8 & 57.1 & 55.5 & 6.1 & 8.0 & 10.4 & 8.3 & 12.9 \\
\hline
\end{tabular}

Notes. The line flux is integrated over the central $20 \mathrm{~km} / \mathrm{s}$ ( $40 \mathrm{~km} / \mathrm{s}$ for $\mathrm{SiO}, 60 \mathrm{~km} / \mathrm{s}$ for b2n and b2m). low means that the $\mathrm{S} / \mathrm{N}$ is too low (non-detection). The baseline subtraction introduces substantial uncertainties. The lower energy level of SiO 8-7 at $347.3 \mathrm{GHz}$ is at $58 \mathrm{~K}$, of $\mathrm{HC}_{3} \mathrm{~N} 38-37$ at $345.6 \mathrm{GHz}$ is at $307 \mathrm{~K}$. ${ }^{(a)} 3-2$ transition ${ }^{(b)} 4-3$ transition

Table 3.5: Velocities of line peaks (in $\mathrm{km} / \mathrm{s}$ ).

\begin{tabular}{lcccccccccccc}
\hline \hline Line & $\mathrm{i} 12$ & $\mathrm{~g} 327$ & $\mathrm{i} 16$ & $\mathrm{ngc}$ & $\mathrm{i} 17$ & $\mathrm{~b} 2 \mathrm{n}$ & $\mathrm{b} 2 \mathrm{~m}$ & $\mathrm{~g} 10$ & $\mathrm{~g} 31$ & $\mathrm{~g} 34$ & w51d & w51e \\
\hline $\mathrm{C}^{18} \mathrm{O} 6-5$ & -39.1 & -44.3 & -62.3 & -7.7 & -2.9 & 66.3 & 62.0 & 66.7 & $\ldots$ & 58.1 & 61.3 & 56.9 \\
$\mathrm{C}^{18} \mathrm{O} 8-7$ & -39.8 & -43.7 & -62.3 & -7.6 & -2.7 & 66.0 & 60.8 & 67.2 & $98.3^{a}$ & 58.2 & 62.0 & 57.6 \\
\hline $\mathrm{HCN} \mathrm{4-3}$ & -40.8 & -48.0 & -57.4 & -10.0 & $\ldots$ & 43.6 & 49.4 & 63.0 & 94.6 & 55.4 & 60.7 & 52.5 \\
$\mathrm{HCN} 8-7$ & -40.6 & -40.2 & -58.7 & -9.9 & 0.5 & 88.8 & 72.3 & 63.1 & $96.3^{b}$ & 57.8 & 61.5 & 52.3 \\
$\mathrm{HCO}^{+} 4-3$ & -40.2 & -47.5 & -64.0 & -7.1 & 0.1 & 46.7 & 49.9 & 63.7 & 95.3 & 56.7 & 61.2 & 52.8 \\
$\mathrm{CO}_{-5}-5$ & -33.6 & -39.7 & -57.7 & -9.9 & 1.4 & 92.0 & 84.6 & 63.2 & $95.8^{c}$ & 55.5 & 59.3 & 51.0 \\
\hline
\end{tabular}

Notes. The $\mathrm{C}^{18} \mathrm{O}$ lines represent the source velocity, the other lines the kinematics (contaminated by the foreground, though). (a) ${ }^{13} \mathrm{CO} 8-7^{(b)} \mathrm{HCN} 9-8^{(c)} \mathrm{CO} 7-6$

Table 3.6: Line widths (full width at half-maximum in $\mathrm{km} / \mathrm{s}$ ) derived from Gaussian fits to the lines.

\begin{tabular}{lccccccccccccc}
\hline \hline Line & i12 & g327 & i16 & ngc & i17 & b2n & b2m & g10 & g31 & g34 & w51d & w51e \\
\hline $\mathrm{HC}^{15} \mathrm{~N} 3-2$ & 6.0 & 9.3 & 8.7 & 8.0 & $\ldots$ & 14.3 & not & 10.7 & $\ldots$ & 7.2 & 9.4 & 11.6 \\
$\mathrm{HC}^{15} \mathrm{~N} 4-3$ & 6.2 & 7.6 & 8.6 & 6.1 & 10.8 & not & 17.9 & 10.2 & 9.0 & $\ldots$ & 6.9 & 10.0 \\
$\mathrm{H}^{13} \mathrm{CN} 3-2$ & 6.4 & not & not & 7.4 & $\ldots$ & not & not & not & $\ldots$ & 6.0 & 7.7 & 11.4 \\
$\mathrm{H}^{13} \mathrm{CN} 4-3$ & 7.4 & not & not & 7.2 & not & not & not & not & 7.8 & 6.1 & 8.5 & not \\
$\mathrm{H}^{13} \mathrm{CN} 8-7$ & 7.3 & 10.3 & 10.3 & 4.5 & 12.0 & not & not & 14.5 & $\ldots$ & 9.0 & 12.7 & not \\
\hline $\mathrm{HC}^{17} \mathrm{O}^{+} 3-2$ & low & $\ldots$ & low & $\ldots$ & $\ldots$ & 7.1 & 23.6 & low & $\ldots$ & 8.5 & low & 8.4 \\
$\mathrm{HC}^{18} \mathrm{O}^{+} 4-3$ & low & low & low & 3.8 & $\ldots$ & not & 25.0 & low & 9.8 & $\ldots$ & low & low \\
$\mathrm{H}^{13} \mathrm{CO}^{+} 3-2$ & 4.6 & $\ldots$ & 4.7 & $\ldots$ & $\ldots$ & not & not & 8.8 & $\ldots$ & 5.7 & 6.7 & 8.0 \\
$\mathrm{H}^{13} \mathrm{CO}^{+} 4-3$ & 5.1 & 5.4 & 4.7 & 4.9 & $\ldots$ & 9.0 & 19.2 & 5.8 & 5.4 & 5.5 & 6.2 & 7.3 \\
$\mathrm{H}^{13} \mathrm{CO}^{+} 8-7$ & $\ldots$ & low & $\ldots$ & low & low & not & 15.5 & low & $\ldots$ & low & 6.4 & low \\
\hline $\mathrm{C}^{17} \mathrm{O} 2-1$ & 4.8 & $\ldots$ & 4.5 & $\ldots$ & $\ldots$ & $\ldots$ & $\ldots$ & $\ldots$ & $\ldots$ & $\ldots$ & $\ldots$ & $\ldots$ \\
$\mathrm{C}^{18} \mathrm{O} 6-5$ & 6.9 & 6.3 & 7.8 & 5.3 & 8.5 & 15.9 & 19.2 & 8.5 & $\ldots$ & 6.5 & 5.6 & 8.1 \\
$\mathrm{C}^{18} \mathrm{O} 8-7$ & 5.3 & 5.0 & 6.6 & 5.1 & 5.5 & 12.9 & 19.9 & 7.3 & $\ldots$ & 6.5 & 5.4 & 7.5 \\
${ }^{13} \mathrm{CO} 8-7$ & not & 7.4 & 10.3 & 6.9 & 11.5 & not & not & 9.9 & 6.1 & 5.9 & 7.9 & 11.8 \\
\hline $\mathrm{HCN}, v_{2}=13-2$ & 6.9 & 6.8 & 11.5 & 5.3 & $\ldots$ & not & 19.2 & 7.5 & low & 4.5 & 8.2 & not \\
$\mathrm{H}^{13} \mathrm{CN}, v_{2}=18-7$ & low & 6.1 & 6.2 & low & low & 16.6 & 20.9 & 11.7 & $\ldots$ & low & low & low \\
\hline
\end{tabular}

Notes. If the line shape differs too significantly from a Gaussian, this is indicated by not. low means that the $\mathrm{S} / \mathrm{N}$ is too low to allow an accurate derivation of the line width. 


\subsection{Observational results}

We present the results that we were able to directly derive from the observations. The spectra of those lines that were modeled $\left(\mathrm{HCN}, \mathrm{HCO}^{+}\right.$, and $\left.\mathrm{CO}\right)$ are shown at the end of the paper. Additional information can best be obtained from the original data, which the interested reader can download ${ }^{5}$.

The most striking result is that we detect vibrationally excited $\mathrm{HCN}$, whose level energies lie over $1000 \mathrm{~K}$ above the ground, in all sources, and even vibrationally excited $\mathrm{H}^{13} \mathrm{CN}$ in half of the sources $(\mathrm{g} 327, \mathrm{i} 16, \mathrm{i} 17, \mathrm{~b} 2 \mathrm{n}$, $\mathrm{b} 2 \mathrm{~m}, \mathrm{~g} 10)$. It is less clear whether we detect vibrationally excited $\mathrm{HC}^{15} \mathrm{~N}$, which is probably present in some sources. In contrast, there is not a single detection (with upper limits of around $0.1 \mathrm{~K}$ ) of vibrationally excited $\mathrm{HCO}^{+}$, which has very similar level structure and line strengths.

Most lines have complicated shapes, with self-absorption often being present in the main isotopologues of $\mathrm{HCN}, \mathrm{HCO}^{+}$, and $\mathrm{CO}$. The notable two exceptions are $\mathrm{i} 12$ and w51d, whose $\mathrm{HCN}$ and $\mathrm{HCO}^{+}$lines are singly peaked. The optical depth of all these lines must be very high, as seen from the other isotopologues. In the selfabsorbed lines, the blue peak is always stronger in ngc, g10, g31, g34, w51e, and w51d, the red peak is always stronger in $\mathrm{i} 17$ and $\mathrm{i} 12$, and both asymmetries occur in $\mathrm{g} 327, \mathrm{i} 16, \mathrm{~b} 2 \mathrm{n}$, and $\mathrm{b} 2 \mathrm{~m}$. Line wings are also a prominent feature of these most optically thick lines. The lower level energies lie at 25 and $150 \mathrm{~K}$ for $\mathrm{HCN}_{\text {and }} \mathrm{HCO}^{+} 4-3$ and 9-8, respectively, and at 33 and $117 \mathrm{~K}$ for $\mathrm{CO} 4-3$ and 7-6, respectively.

To derive more quantitative information, we list the line parameters of selected lines: Table 3.3 compares the intensity ratios, Table 3.4 gives integrated line intensities, Table 3.5 presents the peak velocities, and Table 3.6 contains the line widths. However, we highlight that the optical depths are generally very high, so the lines are severely affected by gradients in excitation temperature, geometry, and velocity field, and can only be understood by means of radiative transfer modeling of the line shapes. For example, even the $\mathrm{HC}^{15} \mathrm{~N} 4-3$ line is optically thick towards the center of all models presented in Sect. 3.8.

\subsection{Modeling procedure: Continuum}

We first attempt to reproduce the radial intensity profile from LABOCA. The temperature in our spherical model with density power law gradient is determined in an approximate way from central heating (Sect. 3.6.1). We set up a grid to cover the relevant ranges of parameters (Sect. 3.6.2), and select the models that are consistent with the continuum data (Sect. 3.6.3).

\subsubsection{Temperature computation}

We use an approximate method to determine the temperature in a spherical, centrally heated cloud with high column density. This method goes back to Larson (1969b) and was also used in a similar way by Osorio et al. (1999).

In its inner parts, the dust is optically thick to its own radiation, which leads to diffusion of radiation. Both the dust temperature and density decrease outwards, both lowering the opacity because the decreasing temperature shifts the radiation field to longer wavelengths where the dust is less opaque. When the radiation is able to escape, a dust photosphere develops. This concept is similar to the effective radius of a star, where the whole luminosity would be emitted at one effective temperature. The photospheric radius separates the inner region, where diffusion determines the temperature gradient, from the outer region, where balance between heating and cooling determines the dust temperature.

The computation is done as follows. First, the Planck and Rosseland mean opacities are computed from tabulated dust opacities by weighting with the Planck function, thus converting frequency-dependent into temperaturedependent opacities. The photospheric radius is given by $R_{\mathrm{ph}}=\sqrt{\frac{L}{4 \pi \sigma T_{\mathrm{ph}}^{4}}}$. At larger radii, the balance between heating and cooling is

$$
T_{\mathrm{ph}}^{4} \kappa_{P}\left(T_{\mathrm{ph}}\right) \Omega=T^{4} \kappa_{P}(T)
$$

where $\Omega=0.5\left(1-\sqrt{1-\left(r / R_{\mathrm{ph}}\right)^{-2}}\right)$ is the solid angle of the photosphere at radius $r$. This allows us to find the radius for any temperature. At smaller radii, it is found by integrating the diffusion equation that

$$
\frac{d r}{d T}=\frac{-16 T^{3} r^{2}}{3 T_{\mathrm{ph}}^{4} \kappa_{R}(T) n(r)} .
$$

The density $n$ is determined by the density power-law index $p$ and the assumption that the Rosseland optical depth at $T_{\mathrm{ph}}$ from the photospheric to the outer radius is $2 / 3$.

\footnotetext{
${ }^{5}$ https://www.astro.uni-koeln.de/wd-schilke/Data/Rolffs
} 
Table 3.7: Average of the $n_{\text {mod }}$ selected models, with standard deviations of the parameters and derived properties (central column density of $\mathrm{H}_{2}$ in a pencil beam, total mass, mass above $100 \mathrm{~K}$, inner, photospheric, and outer radii, density at the photospheric radius).

\begin{tabular}{|c|c|c|c|c|c|c|c|c|c|c|c|c|c|c|c|}
\hline Source & opac & $n_{\text {mod }}$ & $p$ & $\Delta p$ & $\begin{array}{c}L \\
{\left[\mathrm{~L}_{\odot}\right]}\end{array}$ & $\begin{array}{c}\Delta L \\
{\left[\mathrm{~L}_{\odot}\right]}\end{array}$ & $\begin{array}{l}T_{\mathrm{ph}} \\
{[\mathrm{K}]}\end{array}$ & $\begin{array}{c}\Delta T_{\mathrm{ph}} \\
{[\mathrm{K}]}\end{array}$ & $\begin{array}{c}\mathrm{N}_{\mathrm{H}_{2}} \\
\mathrm{~cm}^{-2}\end{array}$ & $\begin{array}{c}\mathrm{M} \\
{\left[\mathrm{M}_{\odot}\right]}\end{array}$ & $\begin{array}{c}\mathrm{M}_{>100 \mathrm{~K}} \\
{\left[\mathrm{M}_{\odot}\right]}\end{array}$ & $\begin{array}{c}\mathrm{R}_{\mathrm{in}} \\
{[\mathrm{AU}]}\end{array}$ & $\begin{array}{c}\mathrm{R}_{\mathrm{ph}} \\
{[\mathrm{AU}]}\end{array}$ & $\begin{array}{l}R_{\text {out }} \\
{[\mathrm{AU}]}\end{array}$ & $\begin{array}{c}\mathrm{n}_{\mathrm{ph}} \\
{\left[\mathrm{cm}^{-3}\right]}\end{array}$ \\
\hline i12 & bare,e6 & 42 & 1.85 & 0.12 & $1.8(5)$ & $6.2(4)$ & 90 & 10 & $5.8(24)$ & $2.0(3)$ & 21 & $2.0(2)$ & $7.9(3)$ & $2.4(5)$ & $9.3(5)$ \\
\hline i12 & bare,no & 48 & 1.80 & 0.15 & $1.7(5)$ & $6.0(4)$ & 67 & 6 & $1.9(25)$ & $1.1(4)$ & 114 & $3.1(2)$ & $1.4(4)$ & $2.2(5)$ & $1.7(6)$ \\
\hline i12 & thin,e6 & 46 & 1.80 & 0.14 & $1.7(5)$ & $6.0(4)$ & 68 & 6 & $7.9(24)$ & $3.7(3)$ & 40 & $2.6(2)$ & $1.4(4)$ & $2.2(5)$ & $6.5(5)$ \\
\hline $\mathrm{i} 12$ & bare,e5 & 43 & 1.81 & 0.14 & $1.7(5)$ & $6.3(4)$ & 78 & 9 & $1.0(25)$ & $4.2(3)$ & 42 & $2.3(2)$ & $1.0(4)$ & $2.2(5)$ & $1.2(6)$ \\
\hline g327 & bare,e6 & 79 & 1.68 & 0.24 & $6.1(4)$ & $2.2(4)$ & 63 & 8 & $8.7(24)$ & $1.8(3)$ & 15 & $1.3(2)$ & $9.5(3)$ & $1.1(5)$ & $1.2(6)$ \\
\hline g327 & bare,no & 56 & 1.61 & 0.26 & $6.6(4)$ & $2.3(4)$ & 50 & 6 & $2.7(25)$ & $9.5(3)$ & 94 & $2.1(2)$ & $1.6(4)$ & $1.0(5)$ & $2.5(6)$ \\
\hline g327 & thin,e6 & 57 & 1.62 & 0.24 & $6.7(4)$ & $2.3(4)$ & 51 & 6 & $1.1(25)$ & $3.4(3)$ & 33 & $1.7(2)$ & $1.5(4)$ & $1.1(5)$ & $9.0(5)$ \\
\hline g327 & bare,e5 & 73 & 1.66 & 0.24 & $6.5(4)$ & $2.2(4)$ & 57 & 7 & $1.5(25)$ & $3.8(3)$ & 35 & $1.6(2)$ & $1.2(4)$ & $1.1(5)$ & $1.6(6)$ \\
\hline i16 & bare,e6 & 43 & 1.83 & 0.12 & $1.9(5)$ & $6.5(4)$ & 106 & 10 & $4.0(24)$ & $1.6(3)$ & 13 & $1.7(2)$ & $6.1(3)$ & $2.9(5)$ & $9.3(5)$ \\
\hline i16 & bare,no & 38 & 1.74 & 0.16 & $1.7(5)$ & $7.0(4)$ & 75 & 8 & $1.3(25)$ & $8.7(3)$ & 61 & $2.5(2)$ & $1.1(4)$ & $2.4(5)$ & $1.7(6)$ \\
\hline i16 & thin,e6 & 36 & 1.74 & 0.15 & $1.7(5)$ & $6.8(4)$ & 75 & 8 & $5.5(24)$ & $3.0(3)$ & 22 & $2.1(2)$ & $1.1(4)$ & $2.3(5)$ & $6.3(5)$ \\
\hline i16 & bare,e5 & 37 & 1.79 & 0.14 & $1.9(5)$ & $6.9(4)$ & 91 & 10 & $6.7(24)$ & $3.6(3)$ & 27 & $2.0(2)$ & $8.1(3)$ & $2.8(5)$ & $1.2(6)$ \\
\hline ngc & bare,e6 & 39 & 1.83 & 0.12 & $1.7(5)$ & $5.7(4)$ & 100 & 9 & $4.4(24)$ & $1.6(3)$ & 14 & $1.7(2)$ & $6.4(3)$ & $2.6(5)$ & $9.7(5)$ \\
\hline $\mathrm{ngc}$ & bare,no & 38 & 1.66 & 0.14 & $1.5(5)$ & $5.4(4)$ & 69 & 7 & $1.3(25)$ & $9.8(3)$ & 56 & $2.2(2)$ & $1.2(4)$ & $2.1(5)$ & $1.6(6)$ \\
\hline $\mathrm{ngc}$ & thin,e6 & 37 & 1.71 & 0.15 & $1.5(5)$ & $5.7(4)$ & 71 & 8 & $5.7(24)$ & $3.2(3)$ & 22 & $2.0(2)$ & $1.2(4)$ & $2.1(5)$ & $6.4(5)$ \\
\hline $\mathrm{ngc}$ & bare,e5 & 28 & 1.79 & 0.11 & $1.8(5)$ & $6.0(4)$ & 87 & 9 & $7.4(24)$ & $3.7(3)$ & 28 & $2.0(2)$ & $8.6(3)$ & $2.6(5)$ & $1.2(6)$ \\
\hline i17 & bare,e6 & 42 & 1.83 & 0.12 & $1.7(4)$ & $6.2(3)$ & 79 & 8 & $7.7(24)$ & $2.7(2)$ & 3 & $7.1(1)$ & $3.2(3)$ & $6.8(4)$ & $2.8(6)$ \\
\hline i17 & bare,no & 42 & 1.79 & 0.15 & $1.8(4)$ & $6.4(3)$ & 61 & 6 & $2.4(25)$ & $1.4(3)$ & 17 & $1.1(2)$ & $5.5(3)$ & $6.5(4)$ & $5.3(6)$ \\
\hline i17 & thin,e6 & 43 & 1.79 & 0.14 & $1.8(4)$ & $6.5(3)$ & 62 & 6 & $9.8(24)$ & $4.9(2)$ & 6 & $9.3(1)$ & $5.3(3)$ & $6.5(4)$ & $2.0(6)$ \\
\hline i17 & bare,e5 & 46 & 1.81 & 0.13 & $1.7(4)$ & $6.0(3)$ & 70 & 7 & $1.3(25)$ & $5.6(2)$ & 6 & $8.4(1)$ & $4.1(3)$ & $6.5(4)$ & $3.7(6)$ \\
\hline $\mathrm{b} 2 \mathrm{n}$ & bare,e6 & 39 & 1.58 & 0.25 & $6.2(5)$ & $1.7(5)$ & 45 & 5 & $1.6(25)$ & $4.1(4)$ & 542 & $5.5(2)$ & $5.9(4)$ & $2.7(5)$ & $3.5(5)$ \\
\hline $\mathrm{b} 2 \mathrm{n}$ & bare,no & 18 & 1.49 & 0.18 & $6.2(5)$ & $1.4(5)$ & 37 & 3 & $4.9(25)$ & $2.1(5)$ & 3303 & $7.9(2)$ & $8.7(4)$ & $2.5(5)$ & $9.9(5)$ \\
\hline $\mathrm{b} 2 \mathrm{n}$ & thin,e6 & 17 & 1.51 & 0.18 & $6.2(5)$ & $1.4(5)$ & 38 & 3 & $2.0(25)$ & $7.4(4)$ & 1196 & $6.8(2)$ & $8.5(4)$ & $2.6(5)$ & $3.5(5)$ \\
\hline $\mathrm{b} 2 \mathrm{n}$ & bare,e5 & 28 & 1.56 & 0.23 & $6.2(5)$ & $1.6(5)$ & 41 & 4 & $2.7(25)$ & $8.4(4)$ & 1262 & $6.6(2)$ & $7.0(4)$ & $2.6(5)$ & $5.4(5)$ \\
\hline b2m & bare,e6 & 57 & 1.58 & 0.13 & $4.0(6)$ & $1.4(6)$ & 69 & 7 & $5.5(24)$ & $1.1(5)$ & 508 & $8.2(2)$ & $6.5(4)$ & $9.4(5)$ & $1.3(5)$ \\
\hline $\mathrm{b} 2 \mathrm{~m}$ & bare,no & 47 & 1.47 & 0.18 & $4.2(6)$ & $1.4(6)$ & 51 & 6 & $1.8(25)$ & $6.1(5)$ & 3080 & $1.2(3)$ & $1.2(5)$ & $8.5(5)$ & $2.8(5)$ \\
\hline $\mathrm{b} 2 \mathrm{~m}$ & thin,e6 & 42 & 1.51 & 0.18 & $4.0(6)$ & $1.5(6)$ & 52 & 6 & $7.5(24)$ & $2.0(5)$ & 1150 & $1.0(3)$ & $1.1(5)$ & $8.5(5)$ & $1.1(5)$ \\
\hline $\mathrm{b} 2 \mathrm{~m}$ & bare,e5 & 49 & 1.57 & 0.15 & $4.0(6)$ & $1.5(6)$ & 61 & 7 & $9.7(24)$ & $2.2(5)$ & 1147 & $1.0(3)$ & $8.3(4)$ & $9.2(5)$ & $1.9(5)$ \\
\hline g10 & bare,e6 & 35 & 1.77 & 0.15 & $5.0(5)$ & $1.3(5)$ & 71 & 6 & $8.5(24)$ & $1.1(4)$ & 112 & $4.0(2)$ & $2.2(4)$ & $3.4(5)$ & $4.6(5)$ \\
\hline g10 & bare,no & 25 & 1.71 & 0.21 & $5.5(5)$ & $1.2(5)$ & 56 & 5 & $2.5(25)$ & $5.6(4)$ & 635 & $6.1(2)$ & $3.6(4)$ & $3.3(5)$ & $9.0(5)$ \\
\hline $\mathrm{g} 10$ & thin,e6 & 25 & 1.76 & 0.18 & $5.5(5)$ & $1.3(5)$ & 57 & 5 & $1.1(25)$ & $2.0(4)$ & 258 & $5.5(2)$ & $3.5(4)$ & $3.4(5)$ & $3.5(5)$ \\
\hline $\mathrm{g} 10$ & bare,e5 & 36 & 1.76 & 0.17 & $5.3(5)$ & $1.3(5)$ & 64 & 6 & $1.4(25)$ & $2.3(4)$ & 247 & $4.8(2)$ & $2.7(4)$ & $3.4(5)$ & $6.1(5)$ \\
\hline g31 & bare,e6 & 58 & 1.53 & 0.27 & $1.9(5)$ & $4.5(4)$ & 68 & 5 & $4.9(24)$ & $5.6(3)$ & 20 & $1.6(2)$ & $1.4(4)$ & $2.0(5)$ & $5.8(5)$ \\
\hline g31 & bare,no & 33 & 1.47 & 0.24 & $2.1(5)$ & $3.6(4)$ & 52 & 4 & $1.7(25)$ & $3.0(4)$ & 142 & $2.7(2)$ & $2.6(4)$ & $1.9(5)$ & $1.2(6)$ \\
\hline g31 & thin,e6 & 36 & 1.48 & 0.26 & $2.1(5)$ & $4.1(4)$ & 52 & 4 & $6.8(24)$ & $1.1(4)$ & 51 & $2.2(2)$ & $2.6(4)$ & $1.9(5)$ & $4.6(5)$ \\
\hline g31 & bare,e5 & 51 & 1.49 & 0.25 & $2.0(5)$ & $4.4(4)$ & 60 & 5 & $8.1(24)$ & $1.2(4)$ & 43 & $1.9(2)$ & $1.9(4)$ & $2.0(5)$ & $7.8(5)$ \\
\hline g34 & bare,e6 & 44 & 1.65 & 0.14 & $2.6(5)$ & $1.0(5)$ & 75 & 8 & $5.4(24)$ & $5.6(3)$ & 30 & $2.1(2)$ & $1.4(4)$ & $2.6(5)$ & $5.8(5)$ \\
\hline g34 & bare,no & 44 & 1.60 & 0.12 & $3.2(5)$ & $1.0(5)$ & 59 & 5 & $1.7(25)$ & $3.1(4)$ & 189 & $3.5(2)$ & $2.5(4)$ & $2.6(5)$ & $1.1(6)$ \\
\hline g34 & thin,e6 & 49 & 1.60 & 0.12 & $3.2(5)$ & $1.1(5)$ & 59 & 5 & $6.6(24)$ & $1.1(4)$ & 66 & $3.0(2)$ & $2.4(4)$ & $2.7(5)$ & $3.9(5)$ \\
\hline g34 & bare,e5 & 57 & 1.60 & 0.12 & $3.0(5)$ & $1.0(5)$ & 68 & 6 & $8.3(24)$ & $1.3(4)$ & 61 & $2.6(2)$ & $1.8(4)$ & $2.7(5)$ & $7.2(5)$ \\
\hline w51d & bare,e6 & 37 & 1.73 & 0.16 & $1.5(6)$ & $5.4(5)$ & 105 & 10 & $3.2(24)$ & $1.5(4)$ & 256 & $4.1(2)$ & $1.7(4)$ & $8.0(5)$ & $3.0(5)$ \\
\hline w51d & bare,no & 48 & 1.58 & 0.12 & $1.5(6)$ & $5.5(5)$ & 74 & 7 & $9.2(24)$ & $9.5(4)$ & 1179 & $5.7(2)$ & $3.4(4)$ & $7.0(5)$ & $4.8(5)$ \\
\hline w51d & thin,e6 & 47 & 1.58 & 0.12 & $1.5(6)$ & $5.5(5)$ & 75 & 7 & $3.6(24)$ & $3.3(4)$ & 415 & $4.7(2)$ & $3.4(4)$ & $6.8(5)$ & $1.8(5)$ \\
\hline w51d & bare,e5 & 38 & 1.63 & 0.14 & $1.5(6)$ & $5.5(5)$ & 89 & 10 & $4.8(24)$ & $3.8(4)$ & 481 & $4.5(2)$ & $2.4(4)$ & $7.6(5)$ & $3.5(5)$ \\
\hline w51e & bare,e6 & 64 & 1.66 & 0.17 & $7.1(5)$ & $2.5(5)$ & 69 & 7 & $6.6(24)$ & $1.8(4)$ & 352 & $3.9(2)$ & $2.7(4)$ & $4.0(5)$ & $3.4(5)$ \\
\hline w51e & bare,no & 39 & 1.58 & 0.13 & $8.3(5)$ & $2.6(5)$ & 54 & 5 & $2.0(25)$ & $9.7(4)$ & 1675 & $6.2(2)$ & 4.7 (4) & $4.0(5)$ & $6.7(5)$ \\
\hline w51e & thin,e6 & 43 & 1.61 & 0.14 & $8.1(5)$ & $2.7(5)$ & 55 & 4 & $8.2(24)$ & $3.4(4)$ & 613 & $5.3(2)$ & $4.5(4)$ & $4.0(5)$ & $2.5(5)$ \\
\hline w51e & bare,e5 & 65 & 1.65 & 0.17 & $8.2(5)$ & $2.7(5)$ & 64 & 6 & $1.1(25)$ & $3.8(4)$ & 732 & $4.9(2)$ & $3.4(4)$ & $4.3(5)$ & $4.5(5)$ \\
\hline
\end{tabular}

This method leads to a discontinuity at $R_{\mathrm{ph}}$, where the temperature rises very slowly inwards and drops by a factor of $\sqrt[4]{0.5}=0.84$ outwards. To overcome this artifact, we interpolate the temperature between $\sqrt[4]{0.5} T_{\mathrm{ph}}$ and $\sqrt[4]{2} T_{\text {ph }}$ as a power law.

In general, the temperature gradient is steeper in the inner parts of the cloud (where the radii are smaller than $\left.R_{\mathrm{ph}}\right)$. One can see this by assuming the behavior of the opacity to be proportional to $\nu^{\beta}$, which renders the mean opacity proportional to $T^{\beta}$, and evaluating the equations of heating/cooling balance and diffusion. The temperature in the outer parts approaches $T_{\mathrm{ph}}\left(\frac{r}{R_{\mathrm{ph}}}\right)^{-q_{\mathrm{out}}}$ with the temperature power law-exponent $q_{\text {out }}=\frac{2}{4+\beta}$. In the inner part, the temperature also follows a power law, but with exponent $q_{\text {in }}=\frac{1+p}{4-\beta}$. With $\beta=1$ and $p=1.5$, one has $q_{\text {out }}=0.4$ and $q_{\text {in }}=0.83$. Using tabulated opacities, the temperature gradients are similar to the these ones.

The following parameters can be set:

- dust opacity (opac): A table with opacities at different wavelengths. 
- density power-law index ( $p)$ : The density varies with radius $r$ as $r^{-p}$.

- photospheric temperature $\left(T_{\mathrm{ph}}\right)$ : The effective temperature of the dust photosphere. It is a measure of the column density (the lower $\mathrm{T}_{\mathrm{ph}}$, the higher the column density).

- inner temperature $\left(T_{\text {in }}\right)$ : The temperature at the inner boundary.

- outer temperature $\left(T_{\text {out }}\right)$ : The temperature at the outer boundary.

- luminosity $(L)$ : The total energy output is not necessary for the computation, but the model size (radius) scales with $\sqrt{L}$.

- distance (dist): To compute the dust continuum flux received on Earth, the distance to the source is needed.

\subsubsection{A grid of models}

We hold the following parameters fixed: the distance at the value given in Table 3.1, the inner temperature at 1500 $\mathrm{K}$ (approximately the dust sublimation temperature), and the outer temperature at $20 \mathrm{~K}$ (temperature of the ambient cloud). An additional four parameters are instead varied:

- Luminosity $(L)$ : We consider values a factor of $0.3-1$ those in Table 3.1 (in steps of 0.1 ). The luminosity in the table is mostly derived from IRAS fluxes, but this more likely reflects the sum of all heating sources within the IRAS beam (there are often other Hit regions close to the hot core). Therefore, the true luminosity can be substantially lower than given in Table 3.1 .

- Photospheric temperature $\left(T_{\mathrm{ph}}\right)$ : To cover a wide range, we try values between 25 and $175 \mathrm{~K}$, in steps of 2.5 $\mathrm{K}$ below $75 \mathrm{~K}$ and in steps of $5 \mathrm{~K}$ above.

- Density gradient $(p)$ : The power-law exponent is varied between 1 and 2.25 in steps of 0.25 .

- Dust opacity (opac): We test four different opacities from Ossenkopf \& Henning (1994), one with thin ice mantles and coagulation at a density of $10^{6} \mathrm{~cm}^{-3}$ (thin,e6), the other three without grain mantles and either no coagulation (bare,no) or coagulation at a density of either $10^{5} \mathrm{~cm}^{-3}$ (bare,e5) or $10^{6} \mathrm{~cm}^{-3}$ (bare,e6)

\subsubsection{Selection}

The SED is contaminated by free-free radiation in the radio and other sources in the far-IR, where the angular resolution is very low and the peak of the emission lies. Therefore, we do not attempt to reproduce the SED, but take its integral as an upper limit to the source luminosity and reproduce only the submm flux, where dust emission from the hot core certainly dominates. For each of the 7680 models, a $345 \mathrm{GHz}$ continuum map is then computed by applying RATRAN. The map is convolved with the APEX beam and radial profiles are extracted using MIRIAD. A model is assumed to successfully reproduce the LABOCA radial profile if the following selection criteria are fulfilled: the peak intensity of the model is between 0.75 and 1.25 times the observational value, its half-power width is smaller than the observational value (since both other sources and observational problems such as an incorrect focus or anomalous refraction tend to broaden the profile), and $\chi_{\text {cont }}^{2}$ is below 1 , with $\chi_{\text {cont }}^{2}=$ $\sum_{i=1}^{30} \frac{\left(o_{i}-m_{i}\right)^{2}}{30\left(\Delta o_{i}^{2}+\left(0.25 o_{i}\right)^{2}\right)}$, where $o_{i}$ and $m_{i}$ are the observed and modeled intensities at $i^{\prime \prime}$ from the center, respectively, and $\Delta o_{i}$ is the rms deviation of the pixels that were averaged to give $o_{i}$.

The selected models $\left(n_{\text {mod }}\right.$ out of 7680) span a cloud in parameter space. For fixed opacity, the parameters are the density gradient $p$, the luminosity $L$, and the photospheric temperature $T_{\mathrm{ph}}$. We take the average (i.e., the center of the parameter cloud) as a good representative of the selected models. Table 3.7 gives the averaged parameters, their standard deviations $\Delta p=\sqrt{\sum_{i=1}^{n_{\bmod }} \frac{\left(p_{i}-p\right)^{2}}{n_{\bmod }}}$ etc., and the most important properties of the models.

\subsection{Modeling procedure: Lines}

We attempt to reproduce the lines from $\mathrm{HCN}, \mathrm{HCO}^{+}$, and $\mathrm{CO}$ by developing detailed radiative-transfer models (Sect. 3.7.1). We explain which continuum-selected models we take as a physical structure to test several chemical structures (Sect. 3.7.2). We then attempt to improve the model fit to the emission lines by varying the abundances of the best-fit model (Sect. 3.7.3). 


\subsubsection{Line radiative transfer}

To compute molecular lines, we use the Monte Carlo radiative-transfer code RATRAN developed by Hogerheijde $\&$ van der Tak (2000). We provide as input the physical structure from the continuum determination, with the gas temperature being equal to the dust temperature. This holds very well for the inner part with its high densities, and lies within the uncertainties for the outer part. The dust mass density is converted to $\mathrm{H}_{2}$ density by assuming a gas-to-dust mass ratio of 100 and that $74 \%$ of the gas mass is in the form of $\mathrm{H}_{2}, 25 \%$ is helium, and $1 \%$ remaining elements). Additionally, the following information is needed:

- Molecular data: Energy levels, transition strengths, and collisional rate coefficients must be provided. For $\mathrm{CO}$ and $\mathrm{HCO}^{+}$isotopologues, we use the entries from the LAMDA database (Schöier et al. 2005), cut above $\mathrm{J}=20\left(\mathrm{HCO}^{+}\right)$and $\mathrm{J}=17(\mathrm{CO})$. For the vibrational bending mode $\left(v_{2}\right)$ of $\mathrm{HCN}$, each $\mathrm{J}$ has two vibrational levels (l-type doubling) about $1000 \mathrm{~K}$ above the ground state (Thorwirth et al. 2003a; Fuchs et al. 2004). Collisional rates for them are unknown, and the computation for so many levels takes a very long time. Furthermore, the accuracy of the Monte Carlo calculations is insufficient for the tiny population difference of the two $l$-type levels, which are very close in energy. Therefore, we use only the first 15 ground-state levels for the Monte Carlo computation and extrapolate the other level populations in LTE relative to the corresponding ground-state J. This method gives very nearly the same results as the complete molecular data file (provided by F. van der Tak), since IR pumping of the ground-state levels operates mainly in regions where the levels are also collisionally excited. Collisional rates for $\mathrm{J}$ up to 8 and temperatures up to $99 \mathrm{~K}$ were provided by Faure and Wernli (2007, priv. comm.); the remainder, with a smooth transition, is from the LAMDA database (extrapolations from Green \& Thaddeus (1974)). Frequencies and Einstein A coefficients are from the Cologne Database for Molecular Spectroscopy (CDMS, Müller et al. 2001, 2005).

- Velocity: The velocity field influences the line shapes. For a centrally heated source, the line profiles are found to be skewed towards the red by an outward motion and towards the blue by an inward motion, since the red side is more absorbed in the latter case. The asymmetry is stronger when the motion is faster (Evans 2003). We ignore the outflow wings, which come from non-spherical motions, by concentrating our attention on the line core. In spherical symmetry, outflows can not be accurately modeled. The velocity field that we use for ngc, b2m, g10, and $\mathrm{g} 31$ is $10 \%$ of free fall, i.e. $\mathrm{v}=-0.1 \times \sqrt{2 G M_{\text {in }} / r}$, where $M_{\text {in }}$ is the mass inside the radius $r$ (starting with $50 \mathrm{M}_{\odot}$ in the center, but $M_{\text {in }}$ is quickly dominated by the surrounding molecular gas). For g34 and w51e, it is $20 \%$ of free fall, while it is static (no velocity field) for the remaining sources (i12, i16, g327, i17, b2n, w51d). Since the modeled lines are not very sensitive to the exact radial behavior of the velocity, we restricted ourselves to fractions of the free-fall velocity; the adopted values are those that reproduce the data best.

- Line width: Internal motions broaden the lines. Interstellar line widths are generally dominated by turbulence or other macroscopic motions such as the relative motions of clumps rather than thermal motions. To reproduce the observed line widths, a 1/e half-width ( 0.6 of FWHM) of $3 \mathrm{~km} / \mathrm{s}(5 \mathrm{~km} / \mathrm{s} \mathrm{FWHM})$ is adopted for all sources except g327 (2.5 km/s; $4 \mathrm{~km} / \mathrm{s} \mathrm{FWHM)} \mathrm{and} \mathrm{b2n} \mathrm{and} \mathrm{b2m} \mathrm{(7} \mathrm{km/s;} 12 \mathrm{~km} / \mathrm{s} \mathrm{FWHM).} \mathrm{This}$ reflects the different degrees of internal motions.

- Abundance: The molecular abundances depend on the chemical evolution. Since modeling this is beyond the scope of the present analysis, we approximate variations with temperature as abundance jumps at certain temperatures. The HCN abundance rises with temperature (Rodgers \& Charnley 2001; Garrod et al. 2008; Boonman et al. 2001), while the $\mathrm{HCO}^{+}$abundance can be reduced at both low and high temperatures (Garrod et al. 2008). For $\mathrm{CO}$, we assume a constant abundance of $10^{-4}$, while we derive the $\mathrm{HCN}$ and $\mathrm{HCO}^{+}$ abundances as described in section 3.7.2.

- Isotope ratios: The isotope ratios determine the relative abundances of the isotopologues, if isotope-sensitive chemistry can be neglected. Based on Wilson \& Rood (1994), we use the following isotope ratios: for the Galactic center sources b2n and b2m ${ }^{12} \mathrm{C} /{ }^{13} \mathrm{C}=20,{ }^{14} \mathrm{~N} /{ }^{15} \mathrm{~N}=600,{ }^{18} \mathrm{O} /{ }^{16} \mathrm{O}=250,{ }^{17} \mathrm{O} /{ }^{16} \mathrm{O}=800$, for the nearby sources i17 and $\operatorname{ngc}{ }^{12} \mathrm{C} /{ }^{13} \mathrm{C}=70,{ }^{14} \mathrm{~N} /{ }^{15} \mathrm{~N}=450,{ }^{18} \mathrm{O} /{ }^{16} \mathrm{O}=550,{ }^{17} \mathrm{O} /{ }^{16} \mathrm{O}=1800$, and for the rest ${ }^{12} \mathrm{C} /{ }^{13} \mathrm{C}=50$, ${ }^{14} \mathrm{~N} /{ }^{15} \mathrm{~N}=400,{ }^{18} \mathrm{O} /{ }^{16} \mathrm{O}=300,{ }^{17} \mathrm{O} /{ }^{16} \mathrm{O}=1000$. The variation with Galactocentric distance is thus approximate.

The output map is convolved with the beam and spectra are extracted using MIRIAD.

\subsubsection{Fitting strategy}

The line computation can only be performed for a limited number of models, as it is highly demanding of computing power. On the basis of previous experience in the modeling, we devised the following general procedure. 
We consider the opacity bare,e5, since that is a compromise between bare,no and bare,e6 and has a similar $850 \mu \mathrm{m}$ opacity as thin,e6. In addition, the dust opacity being the same for every source facilitates a comparison. For this opacity, we consider three models, one close to the average, one with lower density gradient $p$, and one with higher $p$.

For each of the three models, we test freeze-out temperatures of 40 or $50 \mathrm{~K}$ (Garrod et al. 2008), depletion factors of 10,100 , or 1000 , and chemical jump temperatures of 100,150 , or $230 \mathrm{~K}$. Above the latter, $\mathrm{HCO}^{+}$is reduced by a factor of 1000 , while the $\mathrm{HCN}$ abundance is derived from its vibrational excitation. With seven freeze-out possibilities (either no depletion or two temperatures each with three depletion factors) and four hightemperature jump possibilities (no jump or three jump temperatures), there are 28 abundance structures of $\mathrm{HCN}$ and $\mathrm{HCO}^{+}$. The $\mathrm{CO}$ abundance is constant.

For an automatic adjustment of the abundance, an iterative procedure is employed to fit a line of low optical depth: The abundance is multiplied by the ratio of observed to modeled peak intensities; the computation stops when this ratio is close to 1 (within $25 \%$ ) or when five cycles are reached. The line with the lowest optical depth, which is reliably detected, is used. For $\mathrm{HCO}^{+}$, we use the $\mathrm{HC}^{17} \mathrm{O}^{+} 3-2$ line for b2n, b2m, g34, and w51e, $\mathrm{HC}^{18} \mathrm{O}^{+}$ 4-3 for i12, i16, ngc, and $\mathrm{g} 31$, and $\mathrm{H}^{13} \mathrm{CO}^{+} 4-3$ for $\mathrm{g} 327$, i17, g10, and w51d. For HCN, we use the $\mathrm{HC}^{15} \mathrm{~N} 4-3$ line (3-2 in g34) to adjust the abundance below the chemical jump temperature. The abundance at high temperature is traced by $\mathrm{HCN}, v_{2}=13-2$ for i12, ngc, $\mathrm{g} 31$, and w51d, 4-3 for $\mathrm{g} 34$ and w51e, and $\mathrm{H}^{13} \mathrm{CN}, v_{2}=18-7$ for $\mathrm{g} 327$, i16, $\mathrm{i} 17, \mathrm{~b} 2 \mathrm{n}, \mathrm{b} 2 \mathrm{~m}$, and $\mathrm{g} 10$. The starting values are $10^{-10}$ for $\mathrm{HCO}^{+}$and $10^{-9}$ for $\mathrm{HCN}$ between the jump temperatures.

\subsubsection{The 'final' model}

As a figure of merit, we compute for each model and line $\chi_{\text {line }}^{2}=\sum_{i=1}^{n_{\mathrm{ch}}} \frac{\left(o_{i}-m_{i}\right)^{2}}{n_{\mathrm{ch}}\left(0.3 o_{\max }\right)^{2}}$, where $o_{i}$ and $m_{i}$ are the observed and modeled continuum-subtracted intensities, respectively, and $n_{\mathrm{ch}}$ the number of channels between -5 and +5 $\mathrm{km} / \mathrm{s}$ from the source velocity. The observational error is dominated by calibration uncertainties (assumed to be $30 \%$ of the maximum intensity) rather than by thermal noise.

On the basis of a combination of the molecule-averaged $\chi^{2}$ and the normalized $\chi^{2}$ (the latter being the $\chi^{2}$ for the same peak intensities of model and data), we select the best-fit model from the different physical and chemical structures. By modifying the abundances of $\mathrm{HCN}$ and $\mathrm{HCO}^{+}$, we attempt to improve the fit. A constant $\mathrm{CO}$ abundance of $10^{-4}$ is assumed, since modifying this does not lead to significant improvements. This 'final' model is an example of the best-fit solution that can be reached by this method, and is final in the sense that it probably cannot be improved significantly under our assumptions.

\subsection{Modeling results}

We present the results of the fitting strategy. For each source, the 'final' model (parameters in Table 3.8) is compared to the line data, starting with the tighter fits ( $\chi^{2}$ in Table 3.9). Figures 3.2 to 3.13 show the observed lines, superimposed on this model. The rotational lines of the HCN ground vibrational state are shown in the upper left, those of the vibrationally excited state $\left(v_{2}=1\right)$ in the lower left, $\mathrm{HCO}^{+}$lines in the upper right, $\mathrm{CO}$ lines below that, and the lines from the outer pixels of CHAMP+ in the lower right. The vertical scale is the main beam brightness temperature in Kelvin. We note that some lines are affected by blending by neighboring lines, and in some sources broad $\mathrm{CO}$ and $\mathrm{HCN}$ lines blend vibrationally excited $\mathrm{HCN}$ and $\mathrm{HC}^{15} \mathrm{~N}$ (at 265.8, 354.4, 345.8 and 691.5 GHz). This is indicated by a 'B' in the spectrum. Some lines are so strongly blended that they cannot be used and are displayed for illustration purpose only. The strong lines from the main isotoplogues often have outflow wings, which are indicated by an ' $\mathrm{O}$ ' and not modeled. We note that this model is far from being unique, other selected physical structures with adapted abundances are also able to reproduce the data.

This model is compared to the continuum data in Fig. 3.1. The results of the continuum-only modeling are summarized in Table 3.7. Photospheric radii (for radiation of $\approx 40-90 \mathrm{~K}$ ) are around $0.1 \mathrm{pc}$, and the average gas density within it is usually several $10^{6} \mathrm{~cm}^{-3}$ (a few times $10^{5} \mathrm{M}_{\odot} \mathrm{pc}^{-3}$ ). The central optical depth at $850 \mu \mathrm{m}$ can be calculated from the column density $\mathrm{N}_{\mathrm{H}_{2}}$ by multiplying it with $10^{-25}$ and either 1.57 (bare,e6), 0.29 (bare,no), 0.83 (thin,e6), or 0.74 (bare,e5). It is usually between 0.1 and 1 . In addition, we find that $T_{\mathrm{ph}}$ must increase with $L$ and $p$ to fit the observations. In general, mass and bolometric flux are proportional to $L$, the radii to $\sqrt{L}$, and the densities at these radii to $1 / \sqrt{L}$.

\subsubsection{G10.47+0.03}

This massive hot core has optically thick $\mathrm{NH}_{3}(4,4)$ satellites, and expansion motions can be traced by blue-shifted absorption against two hypercompact HII regions (Cesaroni et al. 1994, 1998, 2010). The exceptionally high excitations and column densities of the core were also revealed by the $\mathrm{HC}_{3} \mathrm{~N}$ study of Wyrowski et al. (1999). 

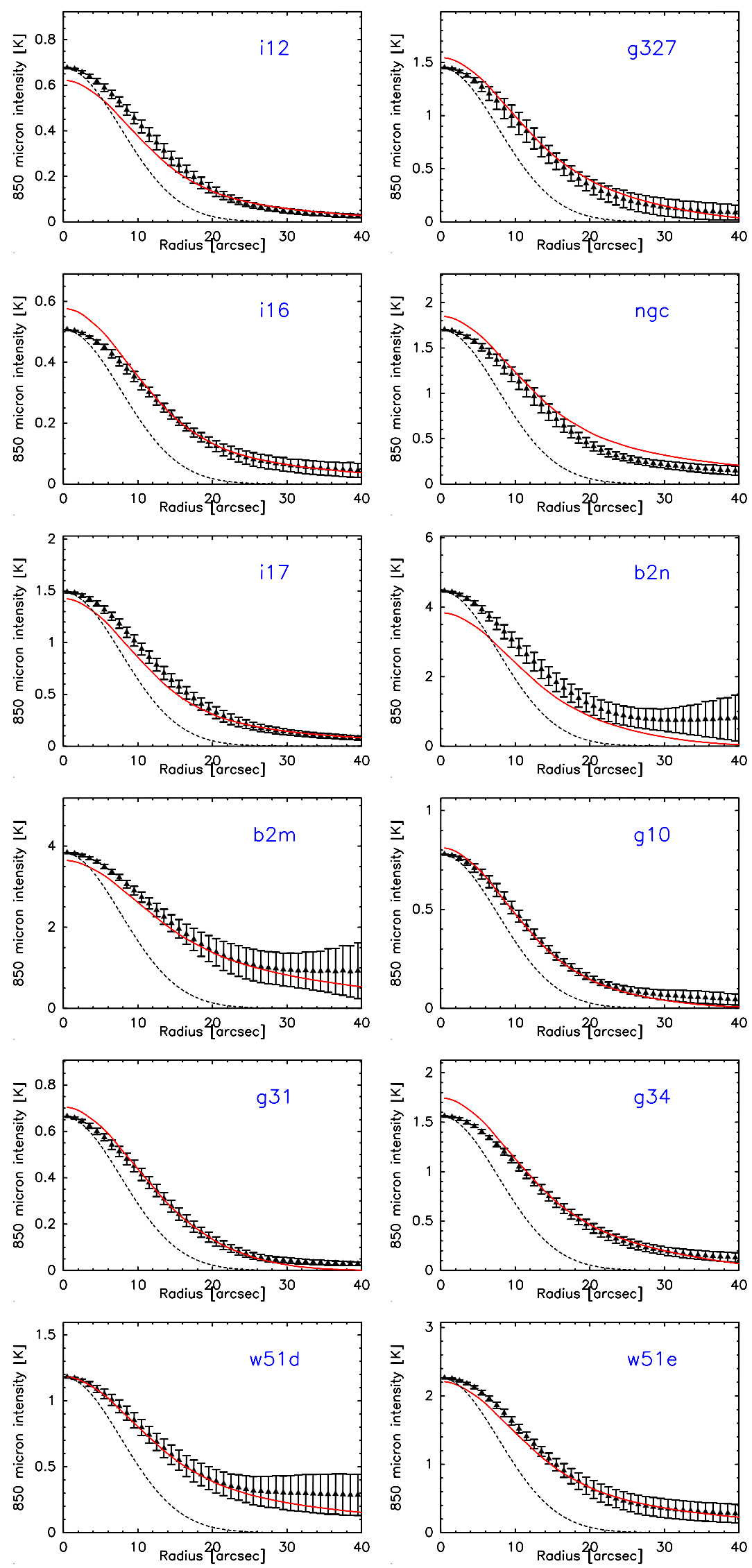

Figure 3.1: Radial intensity profiles at $850 \mu \mathrm{m}$ (LABOCA). The models from Table 3.8 are overlaid in red. The APEX beam is shown as a dashed Gaussian. The errorbars represent only the deviation from a circular shape, an additional error of $25 \%$ in the intensity would account for the calibration uncertainty. 

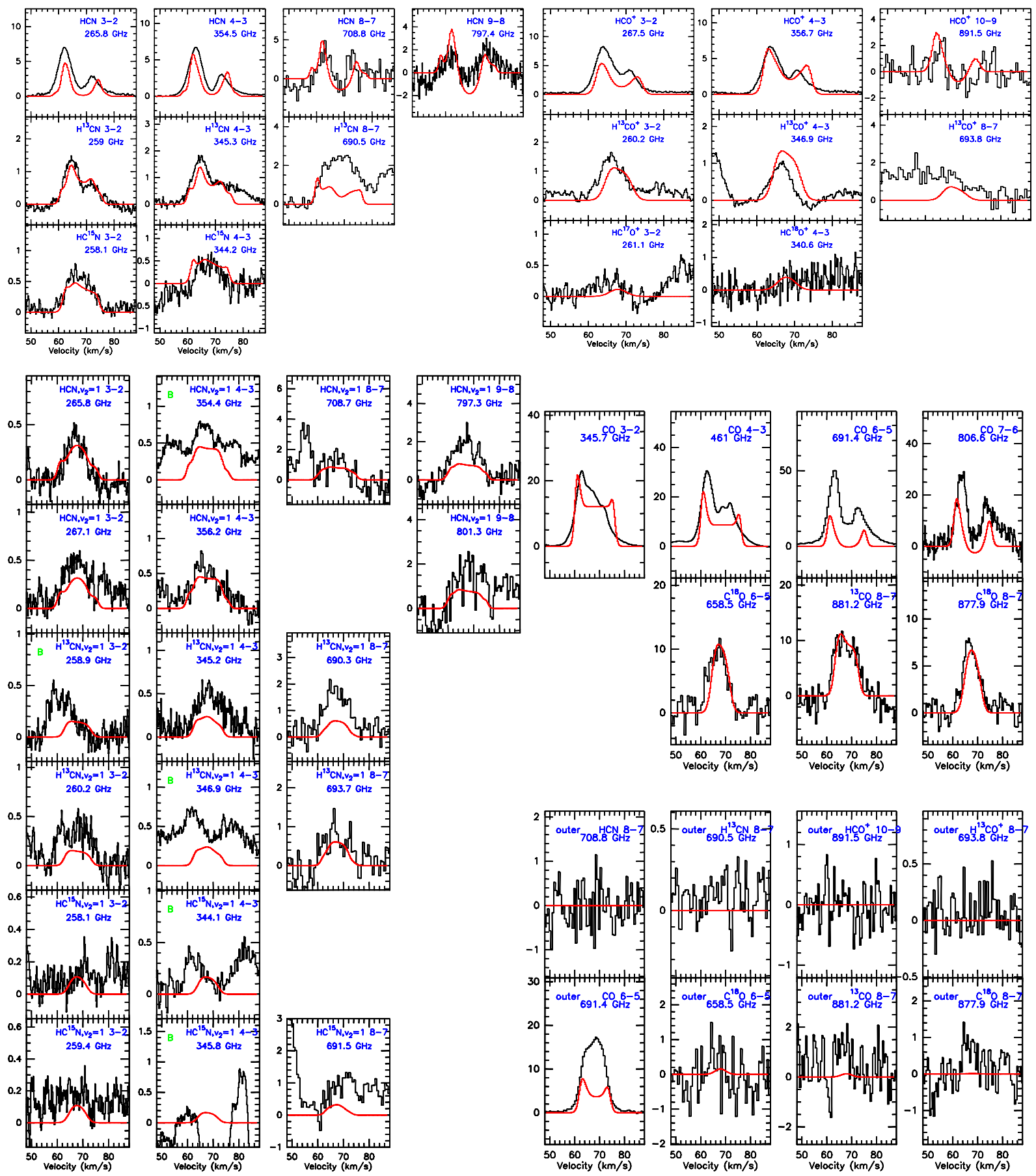

Figure 3.2: Lines towards G10.47+0.03. The model from Table 3.8 is overlaid in red. The lines are ordered on the same grid in all subsequent figures, apparent gaps indicate lines which were not observed. A green 'B' stands for blending by a different line, an 'O' means that the spectrum is likely to be affected by an outflow, which is not included in the model. 
Table 3.8: Parameters of the models that are shown in Figs. 3.2 to 3.13.

\begin{tabular}{|c|c|c|c|c|c|c|c|c|c|c|c|c|c|c|c|c|}
\hline Source & $\mathrm{p}$ & $\mathrm{L}$ & $\mathrm{T}_{\mathrm{ph}}$ & $\mathrm{db}$ & bf & $\overline{\mathrm{A}_{\mathrm{HCN}}^{<T 1}}$ & $T 1$ & $\mathrm{~A}_{\mathrm{HCN}}^{T 1-T 2}$ & $T 2$ & $\overline{\mathrm{A}_{\mathrm{HCN}}^{>T 2}}$ & $\mathrm{~A}_{\mathrm{HCO}^{+}}^{<T 1}$ & $T 1$ & $\mathrm{~A}_{\mathrm{HCO}^{+}}^{T 1-T 2}$ & $T 2$ & $\mathrm{~A}_{\mathrm{HCO}^{+}}^{>T 2}$ & $\mathrm{~A}_{\mathrm{CO}}$ \\
\hline i12 & 1.75 & $1.6(5)$ & 80.0 & 3 & 0 & $2(-10)$ & 50 & $2(-7)$ & $\ldots$ & $\ldots$ & $4(-12)$ & 40 & $4(-9)$ & $\ldots$ & $\ldots$ & $1(-4)$ \\
\hline g327 & 1.50 & $7.0(4)$ & 55.0 & 2.5 & 0 & $5(-10)$ & 40 & $5(-8)$ & 100 & $5(-5)$ & $2(-10)$ & 40 & $2(-9)$ & $\ldots$ & $\ldots$ & $1(-4)$ \\
\hline i16 & 1.75 & $2.0(5)$ & 90.0 & 3 & 0 & $8(-10)$ & 40 & $8(-8)$ & 100 & $1(-6)$ & $7(-11)$ & 40 & $7(-9)$ & $\ldots$ & $\ldots$ & $1(-4)$ \\
\hline ngc & 1.50 & $7.8(4)$ & 65.0 & 3 & 0.1 & $4(-10)$ & 50 & $4(-8)$ & $\ldots$ & $\ldots$ & $2(-11)$ & 50 & $2(-9)$ & 100 & $2(-12)$ & $1(-4)$ \\
\hline i17 & 1.75 & $1.6(4)$ & 70.0 & 3 & 0 & $1.5(-9)$ & 50 & $1.5(-7)$ & 100 & $1(-6)$ & $3(-10)$ & 40 & $3(-9)$ & $\ldots$ & $\ldots$ & $1(-4)$ \\
\hline$b 2 n$ & 1.50 & $5.9(5)$ & 42.5 & 7 & 0 & $5(-9)$ & 40 & $5(-8)$ & 100 & $8(-6)$ & $2(-9)$ & 40 & $2(-8)$ & $\ldots$ & $\ldots$ & $1(-4)$ \\
\hline $\mathrm{b} 2 \mathrm{~m}$ & 1.25 & $1.9(6)$ & 47.5 & 7 & 0.1 & $1.5(-8)$ & $\ldots$ & $\ldots$ & 150 & $5(-6)$ & $5(-10)$ & 40 & $5(-9)$ & $\ldots$ & $\ldots$ & $1(-4)$ \\
\hline g10 & 1.75 & $5.6(5)$ & 65.0 & 3 & 0.1 & $5(-9)$ & 40 & $5(-8)$ & 100 & $3(-5)$ & $8(-10)$ & 40 & $8(-9)$ & $\ldots$ & $\ldots$ & $1(-4)$ \\
\hline g31 & 1.50 & $2.1(5)$ & 60.0 & 3 & 0.1 & $4(-9)$ & 50 & $4(-7)$ & $\ldots$ & $\ldots$ & $2(-9)$ & $\ldots$ & $\ldots$ & $\ldots$ & $\ldots$ & $1(-4)$ \\
\hline g34 & 1.50 & $1.4(5)$ & 55.0 & 3 & 0.2 & $1(-9)$ & 50 & $1(-7)$ & $\ldots$ & $\ldots$ & $3(-9)$ & $\ldots$ & $\ldots$ & 150 & $3(-12)$ & $1(-4)$ \\
\hline w51d & 1.50 & $9.6(5)$ & 80.0 & 3 & 0 & $1(-10)$ & 50 & $1(-7)$ & $\ldots$ & $\ldots$ & $6(-12)$ & 40 & $6(-9)$ & $\ldots$ & $\ldots$ & $1(-4)$ \\
\hline w51e & 1.50 & $7.2(5)$ & 60.0 & 3 & 0.2 & $5(-11)$ & 40 & $5(-8)$ & $\ldots$ & $\ldots$ & $1(-11)$ & 50 & $1(-8)$ & $\ldots$ & $\ldots$ & $1(-4)$ \\
\hline
\end{tabular}

Notes. The opacity is always bare,e5. $\mathrm{p}$ is the density power-law index, $\mathrm{L}$ the luminosity, $\mathrm{T}_{\mathrm{ph}}$ the photospheric temperature, $\mathrm{db}$ is the turbulent $1 / \mathrm{e}$ half-width, bf the fraction of free-fall, and A the molecular fractional abundance. The abundances may jump at temperatures $\mathrm{T} 1$ and $\mathrm{T} 2$.

Table 3.9: $\chi^{2}$ of the models that are shown in Figs. 3.2 to 3.13.

\begin{tabular}{lccccccc}
\hline \hline Source & Fig. & $\chi_{\text {cont }}^{2}$ & $\chi_{\mathrm{HCN}, \mathrm{v}=0}^{2}$ & $\chi_{\mathrm{HCN}, \mathrm{v}_{2}=1}^{2}$ & $\chi_{\mathrm{HCO}^{+}}^{2}$ & $\chi_{\mathrm{CO}}^{2}$ & $\chi_{\text {lines }}^{2}$ \\
\hline i12 & 3.7 & 0.20 & 2.3 & 2.0 & 2.2 & 2.6 & 2.24 \\
g327 & 3.4 & 0.09 & 2.1 & 1.6 & 1.9 & 1.6 & 1.77 \\
i16 & 3.3 & 0.06 & 1.7 & 1.7 & 1.1 & 2.4 & 1.75 \\
ngc & 3.9 & 0.95 & 2.2 & 2.9 & 1.5 & 2.8 & 2.48 \\
i17 & 3.8 & 0.13 & 1.9 & 2.6 & 2.4 & 2.2 & 2.26 \\
b2n & 3.13 & 0.74 & 13.5 & 2.5 & 19.6 & 10.2 & 10.38 \\
b2m & 3.12 & 0.05 & 2.7 & 2.5 & 7.8 & 8.5 & 4.95 \\
g10 & 3.2 & 0.11 & 2.0 & 1.6 & 1.4 & 1.9 & 1.71 \\
g31 & 3.6 & 0.10 & 1.1 & 1.9 & 2.4 & 3.4 & 2.07 \\
g34 & 3.5 & 0.05 & 1.9 & 1.9 & 2.0 & 2.0 & 1.98 \\
w51d & 3.11 & 0.04 & 2.4 & 3.4 & 1.8 & 3.4 & 2.82 \\
w51e & 3.10 & 0.03 & 3.3 & 2.2 & 2.5 & 2.2 & 2.50 \\
\hline
\end{tabular}

Notes. For the lines, $\chi^{2}$ is evaluated over the inner $10 \mathrm{~km} / \mathrm{s}$, assuming an error of $30 \%$ of the peak intensity of the data.

Olmi et al. (1996) detected different velocity gradients in $\mathrm{CH}_{3} \mathrm{CN}$ and ${ }^{13} \mathrm{CO}$, which are possibly indicative of rotation and outflow, respectively.

Figure 3.2 shows the observed lines and the best-fit model (Table 3.8). The line asymmetries are reproduced by a $10 \%$ free-fall contribution. Vibrationally excited $\mathrm{H}^{13} \mathrm{CN}$ is clearly detected, requiring a high abundance in the inner part $\left(3 \times 10^{-5}\right.$ of $\left.\mathrm{H}_{2}\right)$. Owing to the large distance, the outer pixels are weak in this source. While the model fits quite well to the APEX data, our own SMA data reveal that this kind of model does not reproduce the innermost structure, since it is too strongly peaked (Rolffs et al., in prep.).

\subsubsection{IRAS 16065-5158}

Walsh et al. (1998) report the detection of an ultracompact HII region associated with this source. The hot core was detected with the APEX telescope from a large sample of color-selected IRAS sources (Dedes et al. 2011)

Figure 3.3 shows the observed lines and the best-fit model (Table 3.8). The source is probably less concentrated than the model, since the 8-7 transitions of $\mathrm{HCN}$ and $\mathrm{H}^{13} \mathrm{CN}$ do not show self-absorption and the emission in the outer pixels is much stronger than modeled. We note that vibrationally excited $\mathrm{H}^{13} \mathrm{CN}$ and even $\mathrm{HC}^{15} \mathrm{~N}(8-7$ at $691.5 \mathrm{GHz}$ ) is detected here.

\subsubsection{G327.3-0.6}

G327.3-0.6 has a spectacular richness of lines with relatively narrow widths (Gibb et al. 2000; Schilke et al. 2006). The hot core is embedded in a larger structure, where different stages of star formation take place (Wyrowski et al. 2006; Minier et al. 2009). 

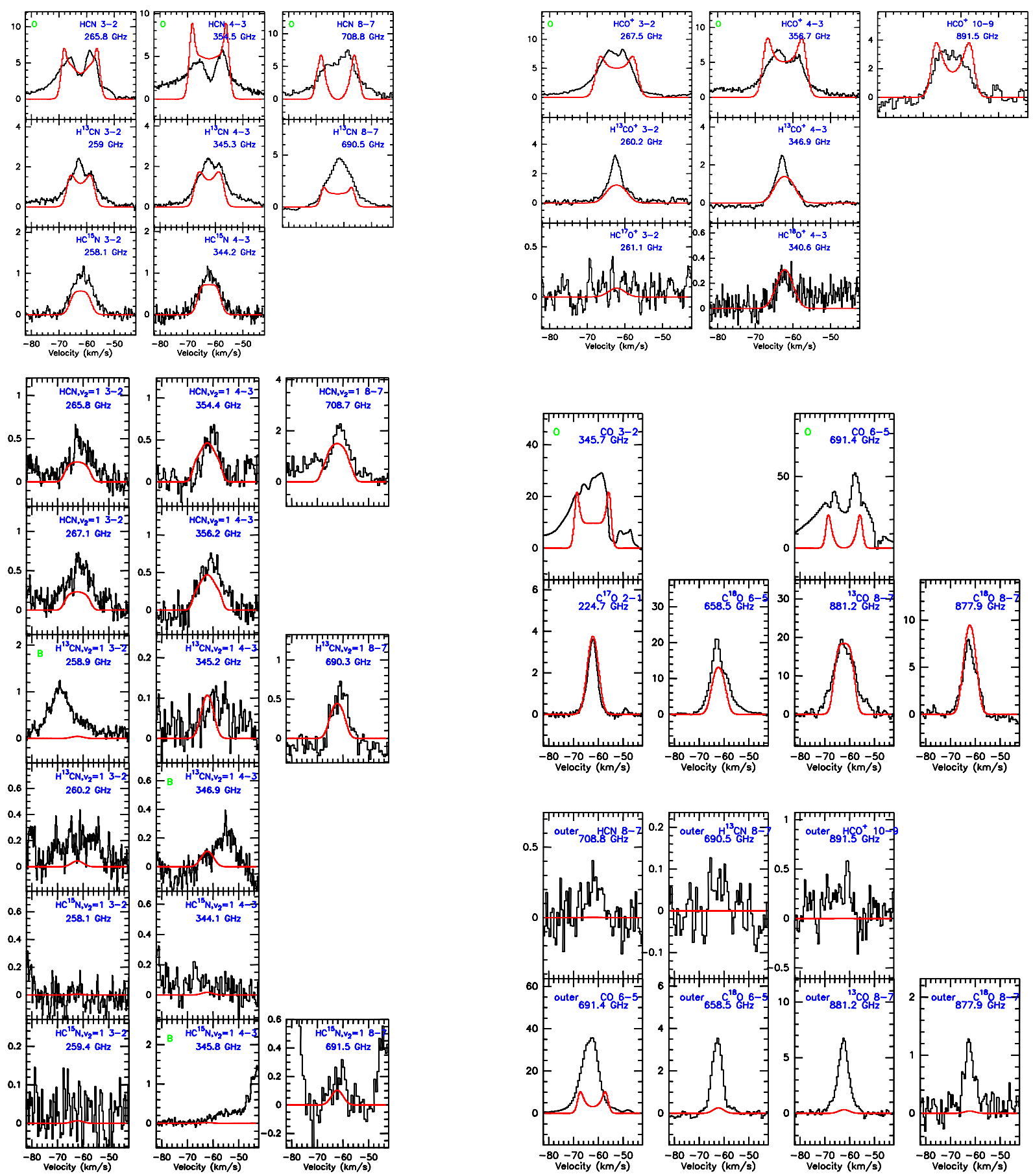

Figure 3.3: Lines towards IRAS 16065-5158. The model from Table 3.8 is overlaid in red. 


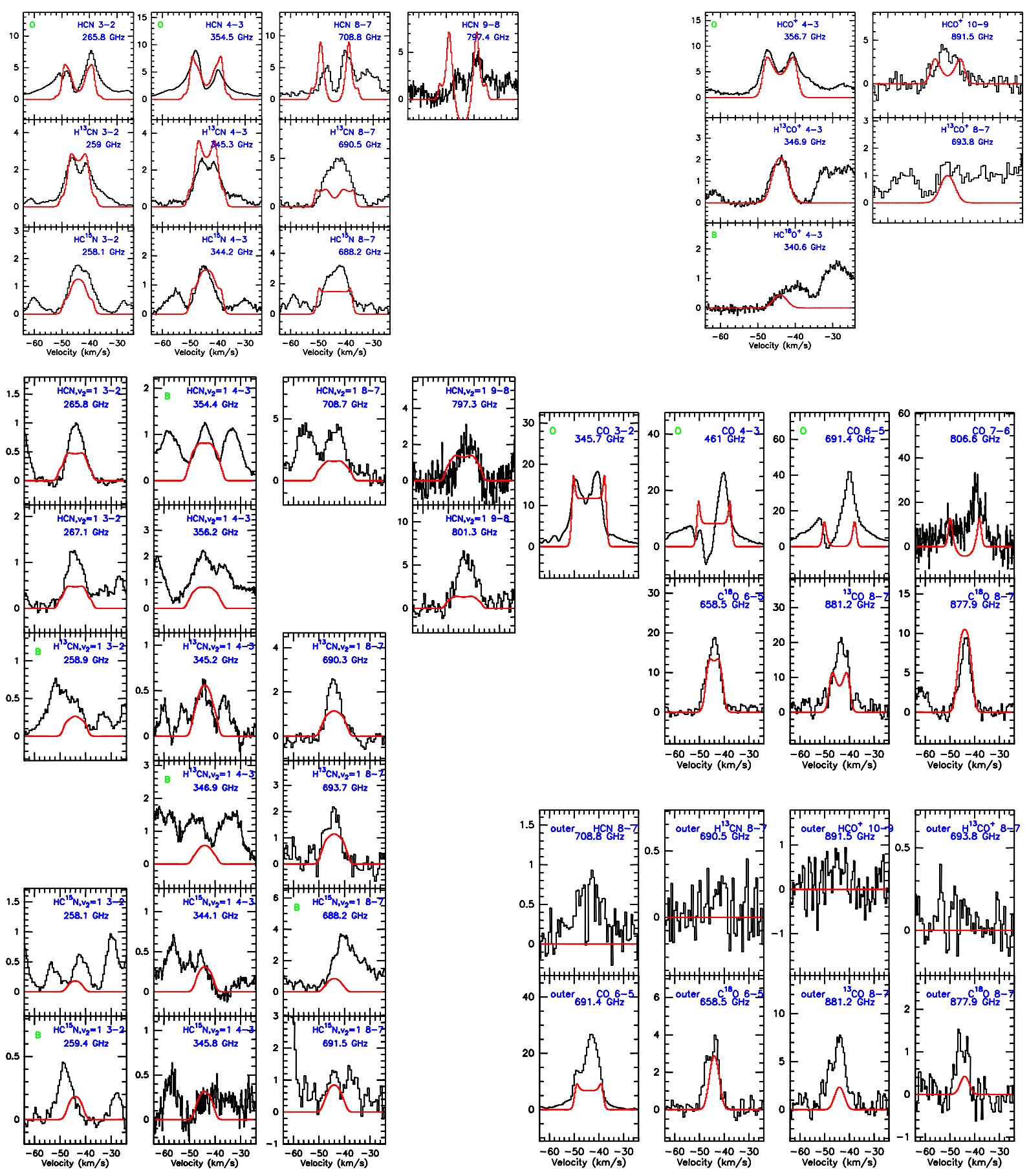

Figure 3.4: Lines towards G327.3-0.6. The model from Table 3.8 is overlaid in red. 

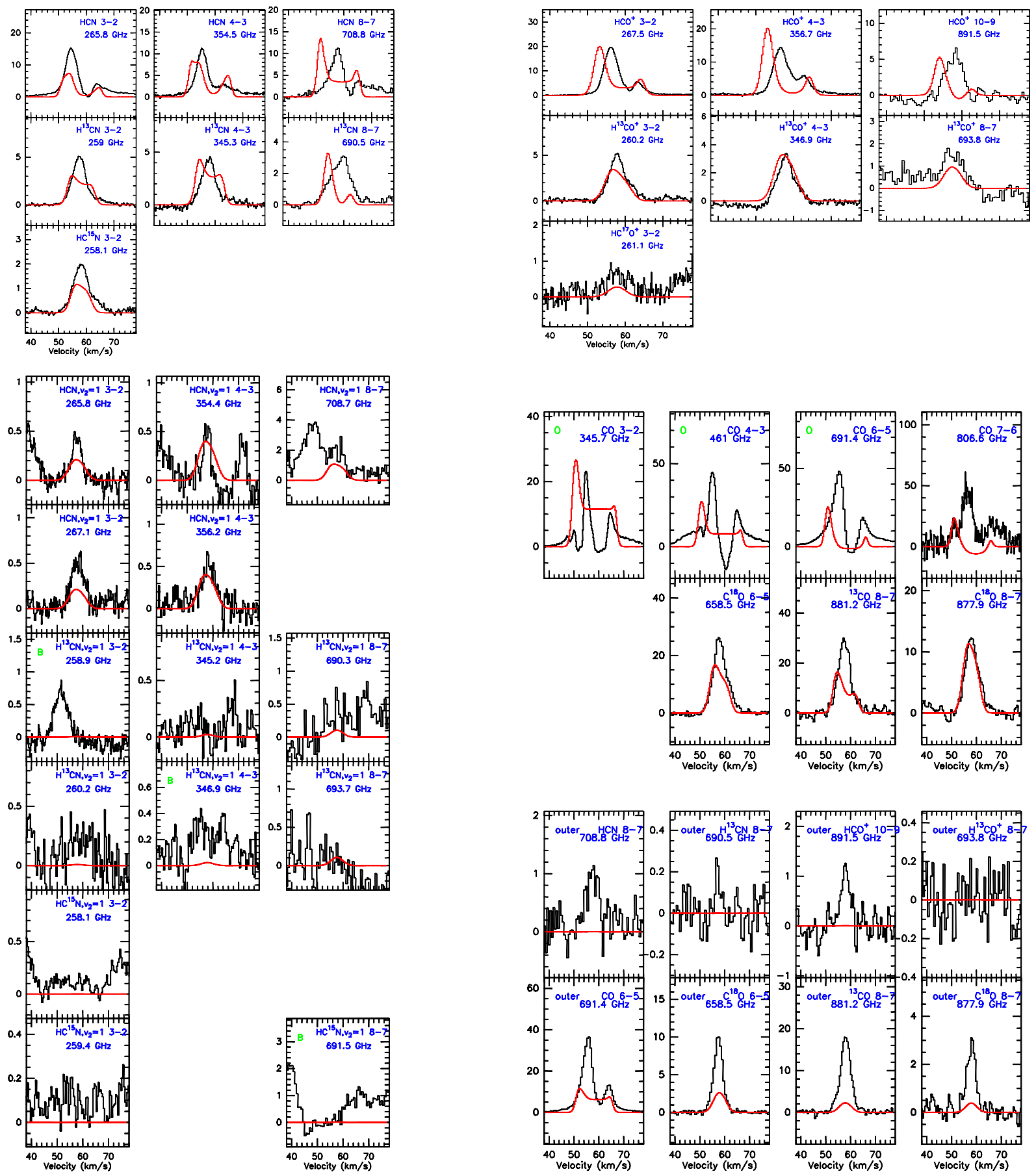

Figure 3.5: Lines towards G34.26+0.15. The model from Table 3.8 is overlaid in red.

Figure 3.4 shows the observed lines and the best-fit model (Table 3.8). The presence of both red and blue asymmetries indicates a complex velocity field. Vibrationally excited HCN, including its isotopologues, is very prominent in this source.

\subsubsection{G34.26+0.15}

This hot core lies off the bow of a cometary-shaped UCHII region (Watt \& Mundy 1999). Therefore, it has been proposed to be externally heated by this HII region (Mookerjea et al. 2007, and references therein). In this scenario, the emission from warm molecules arises in a relatively thin layer (PDR), while the inner cloud regions are cold.

However, we see no evidence for this in our data. Self-absorption and strong red asymmetries instead point to an infalling source with internal heating. We are perhaps able to probe larger scales, so external heating could be internal for our beam size. Figure 3.5 shows the observed lines and the best-fit model (Table 3.8). 

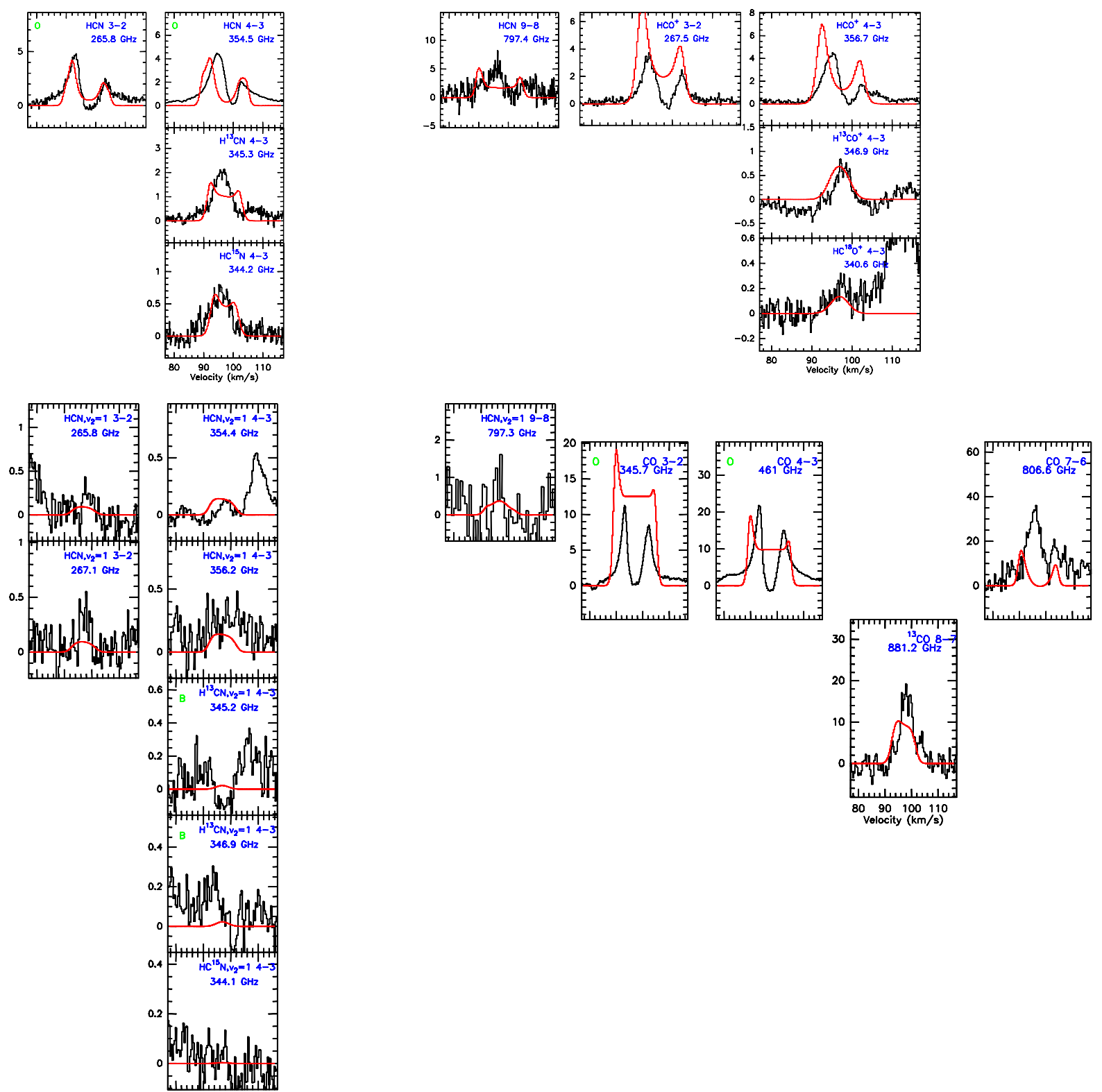

Figure 3.6: Lines towards G31.41+0.31. The model from Table 3.8 is overlaid in red.

\subsubsection{G31.41+0.31}

This hot core has a bipolar outflow (Olmi et al. 1996) and two continuum peaks (Cesaroni et al. 2010). A velocity gradient is interpreted as a massive toroidal disk by Beltrán et al. (2005) and an outflow by Araya et al. (2008).

Figure 3.6 shows the observed lines and the best-fit model (Table 3.8). No CHAMP+ observations were made for this source. With our data, we cannot distinguish between the proposed small-scale velocity fields.

\subsubsection{IRAS 12326-6245}

This hot core is associated with a massive molecular outflow and various infrared and radio sources (Henning et al. 2000).

Figure 3.7 shows the observed lines and the best-fit model (Table 3.8). The lack of self-absorption towards this source cannot be explained in the framework of our spherical models. To weaken self-absorption, the best-fit 

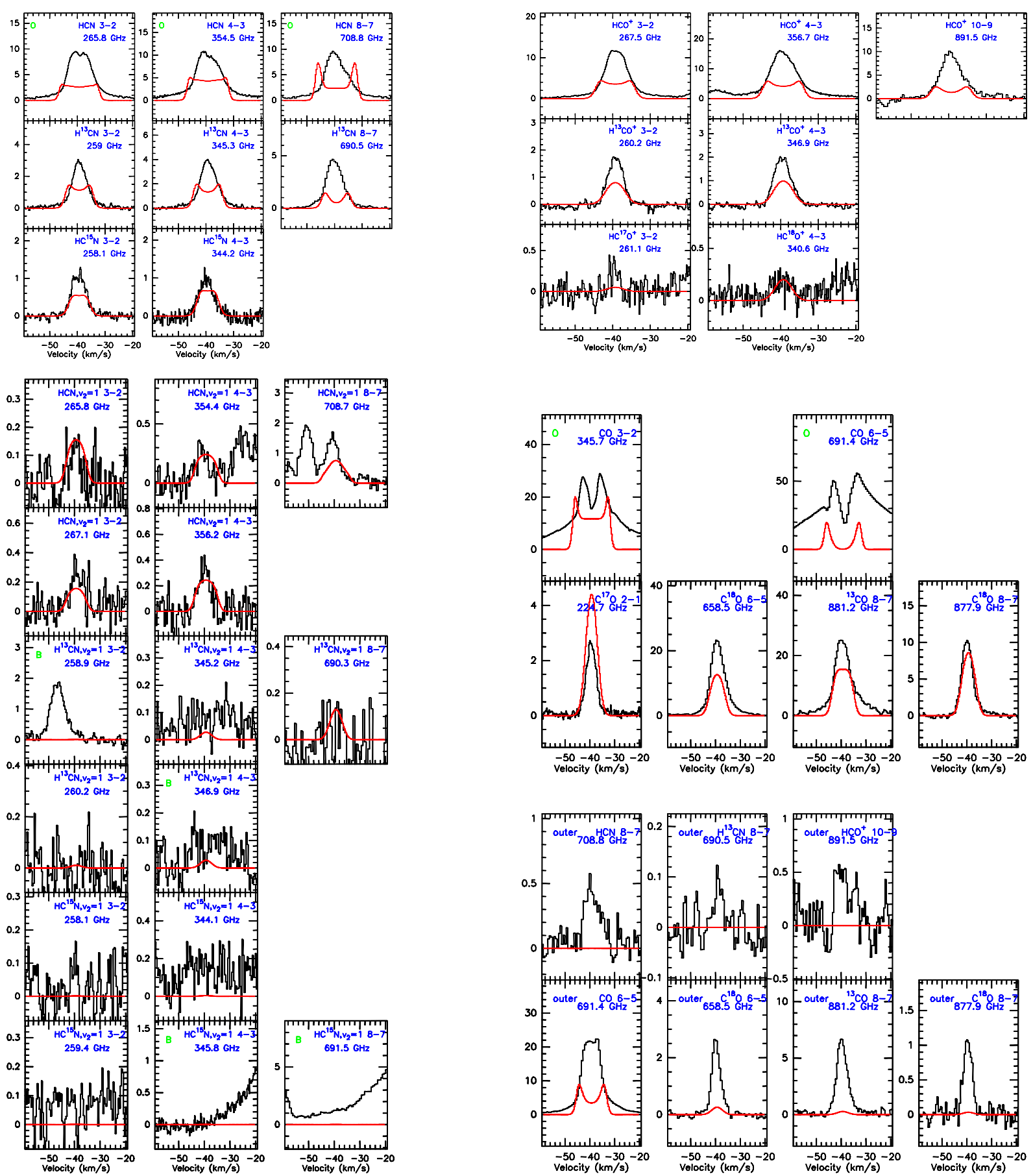

Figure 3.7: Lines towards IRAS 12326-6245. The model from Table 3.8 is overlaid in red. 

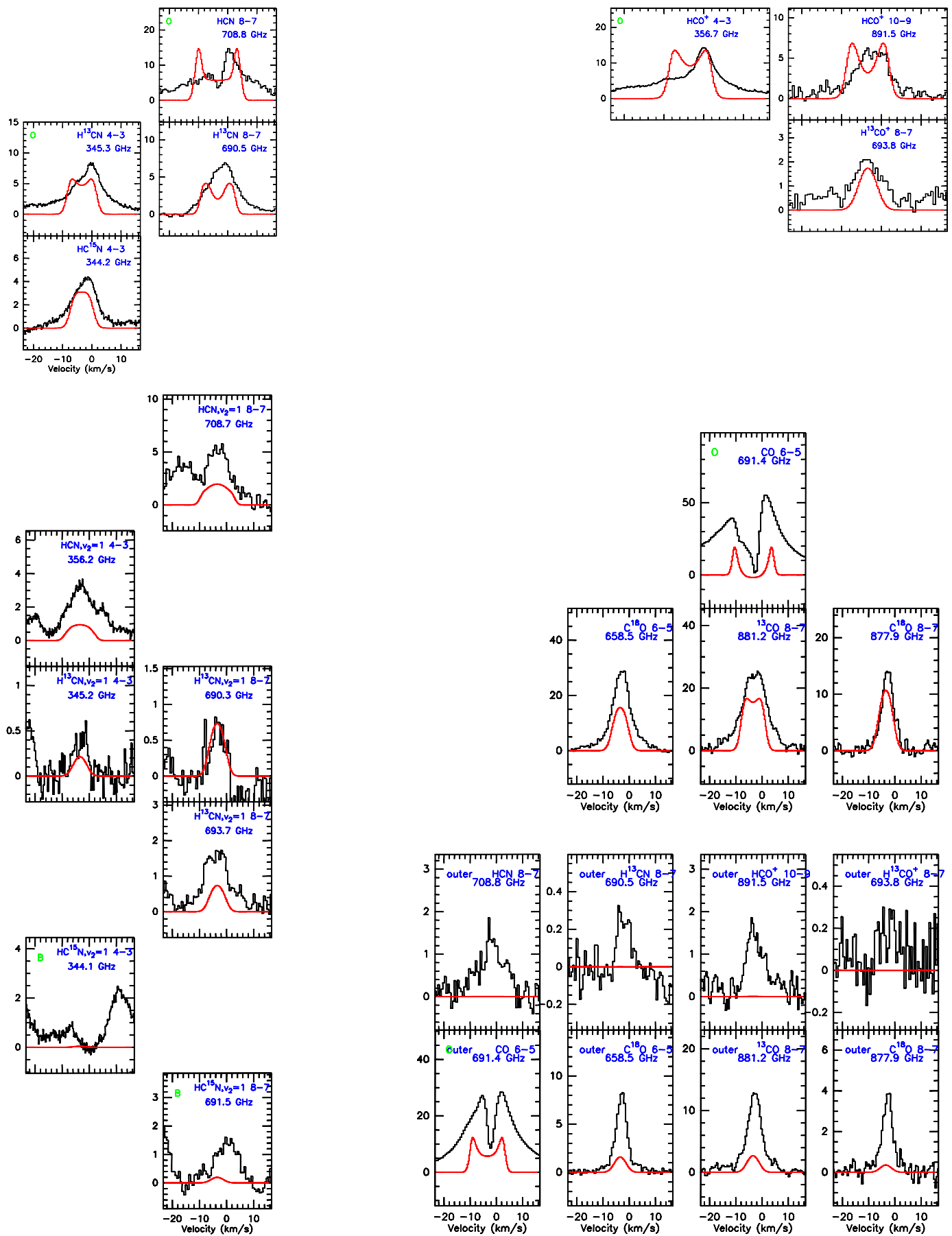

Figure 3.8: Lines towards IRAS 17233-3606. The model from Table 3.8 is overlaid in red.

models have high freeze-out; but abundance variations at higher temperatures are not necessary. In addition, the outer pixels of CHAMP+ have much stronger line emission than modeled.

\subsubsection{IRAS 17233-3606}

IRAS 17233-3606 is a recently detected hot core (Leurini et al. 2008) with a close distance. It drives at least three outflows (Leurini et al. 2009), contains multiple radio sources (Zapata et al. 2008a), and displays a velocity gradient, which is interpreted by Beuther et al. (2009) as rotation, but could also be due to the superposition of outflows.

Figure 3.8 shows the observed lines and the best-fit model (Table 3.8). Broad line wings and blue asymmetries are probably caused by the outflows. In addition, the model has too much self-absorption and too little high-J line emission in the outer pixels. We see strong vibrationally excited $\mathrm{H}^{13} \mathrm{CN}$. 


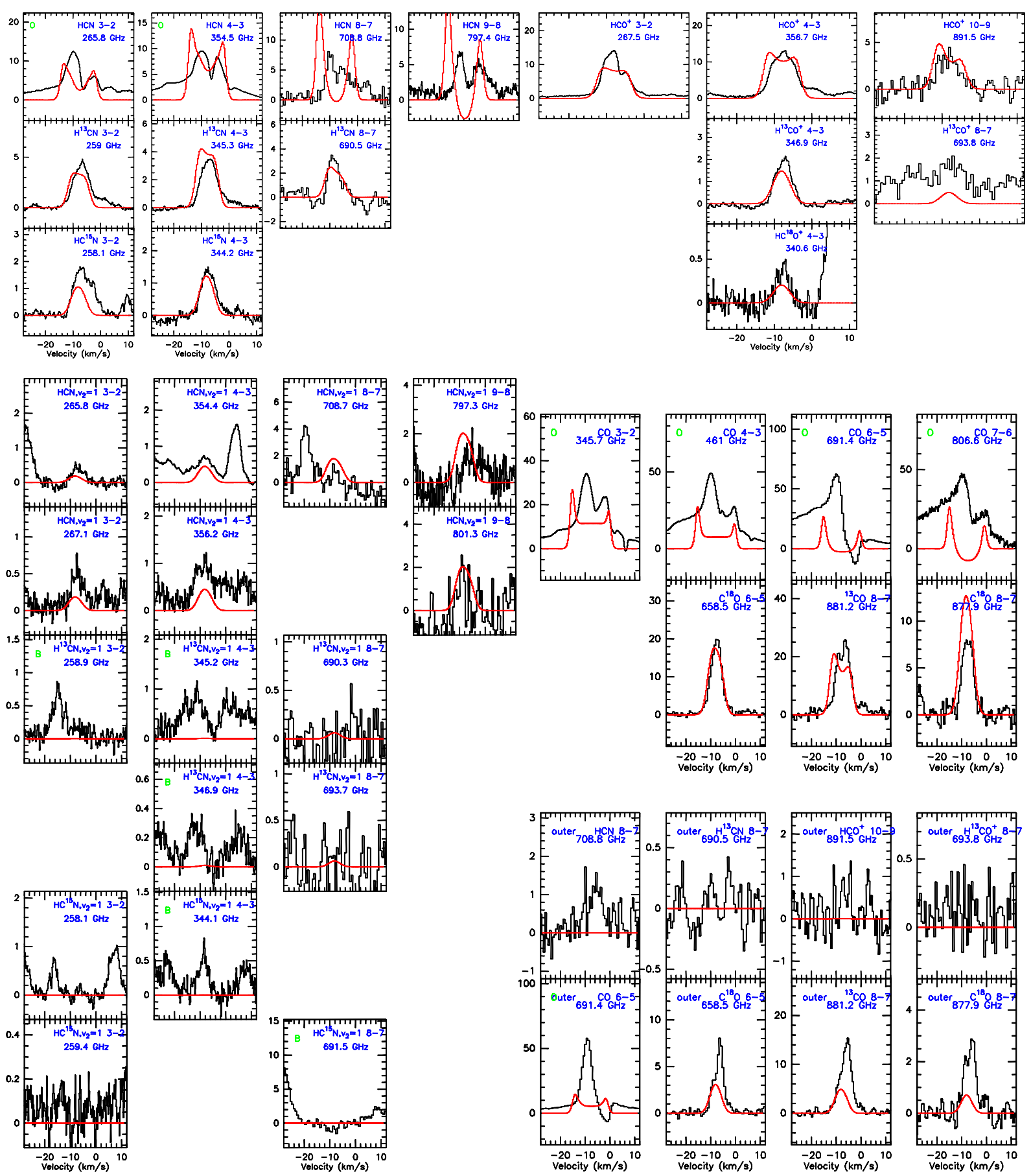

Figure 3.9: Lines towards NGC6334-I. The model from Table 3.8 is overlaid in red.

\subsubsection{NGC6334-I}

NGC6334-I was extensively studied in line surveys (Thorwirth et al. 2007; Schilke et al. 2006). The SMA resolves the core into several $\mathrm{mm}$ peaks (Hunter et al. 2006), which show very different emission-line characteristics (Brogan et al. 2009). The large-scale environment was mapped by Walsh et al. (2010), and an outflow is also present (Beuther et al. 2008). This source was observed with Herschel/HIFI (Emprechtinger et al. 2010; Ceccarelli et al. 2010).

Figure 3.9 shows the observed lines and the best-fit model (Table 3.8). Owing, possibly, to the clumpiness, the high-J HCN lines are weaker and less self-absorbed than in the model. 


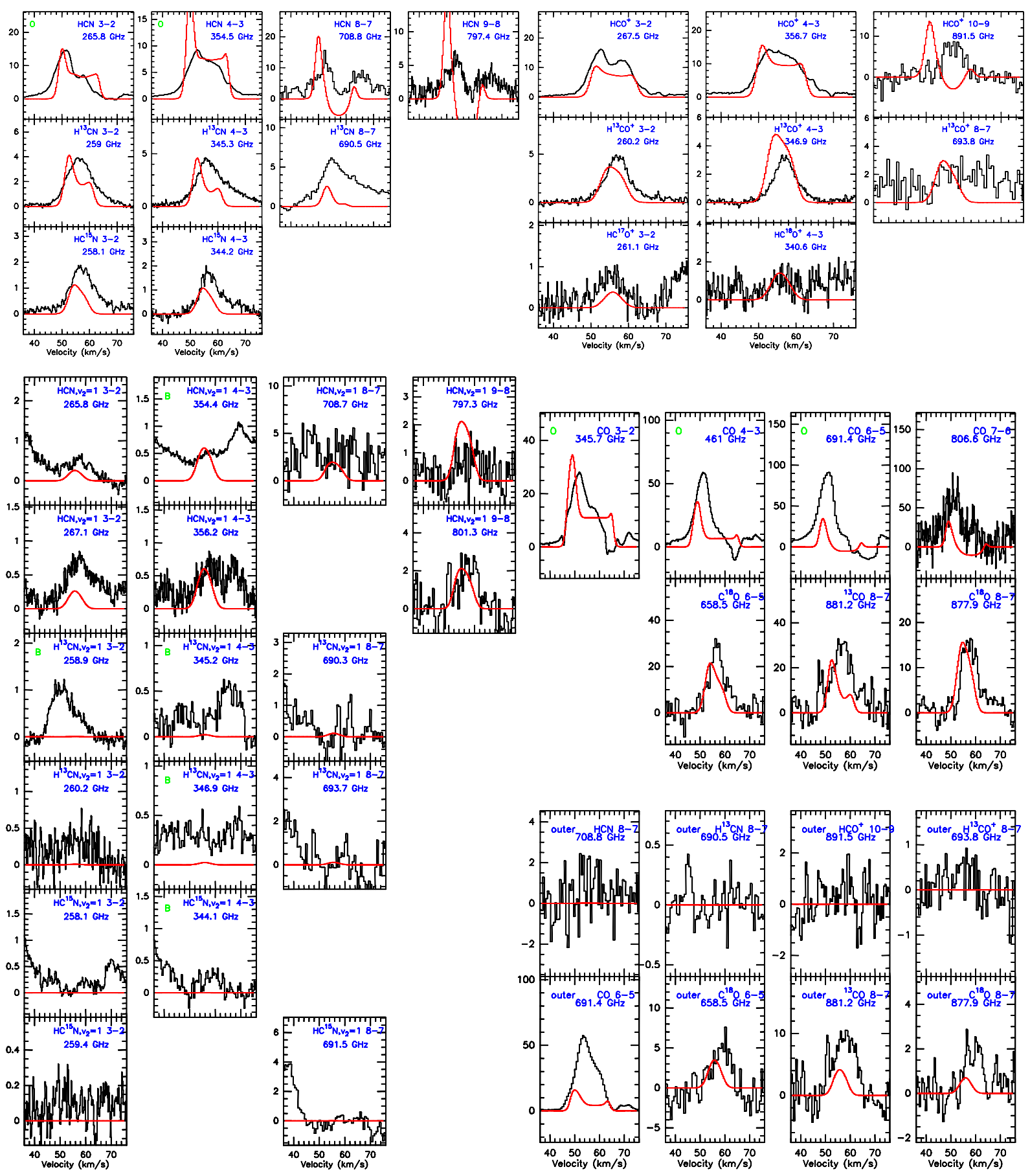

Figure 3.10: Lines towards W51e. The model from Table 3.8 is overlaid in red. 

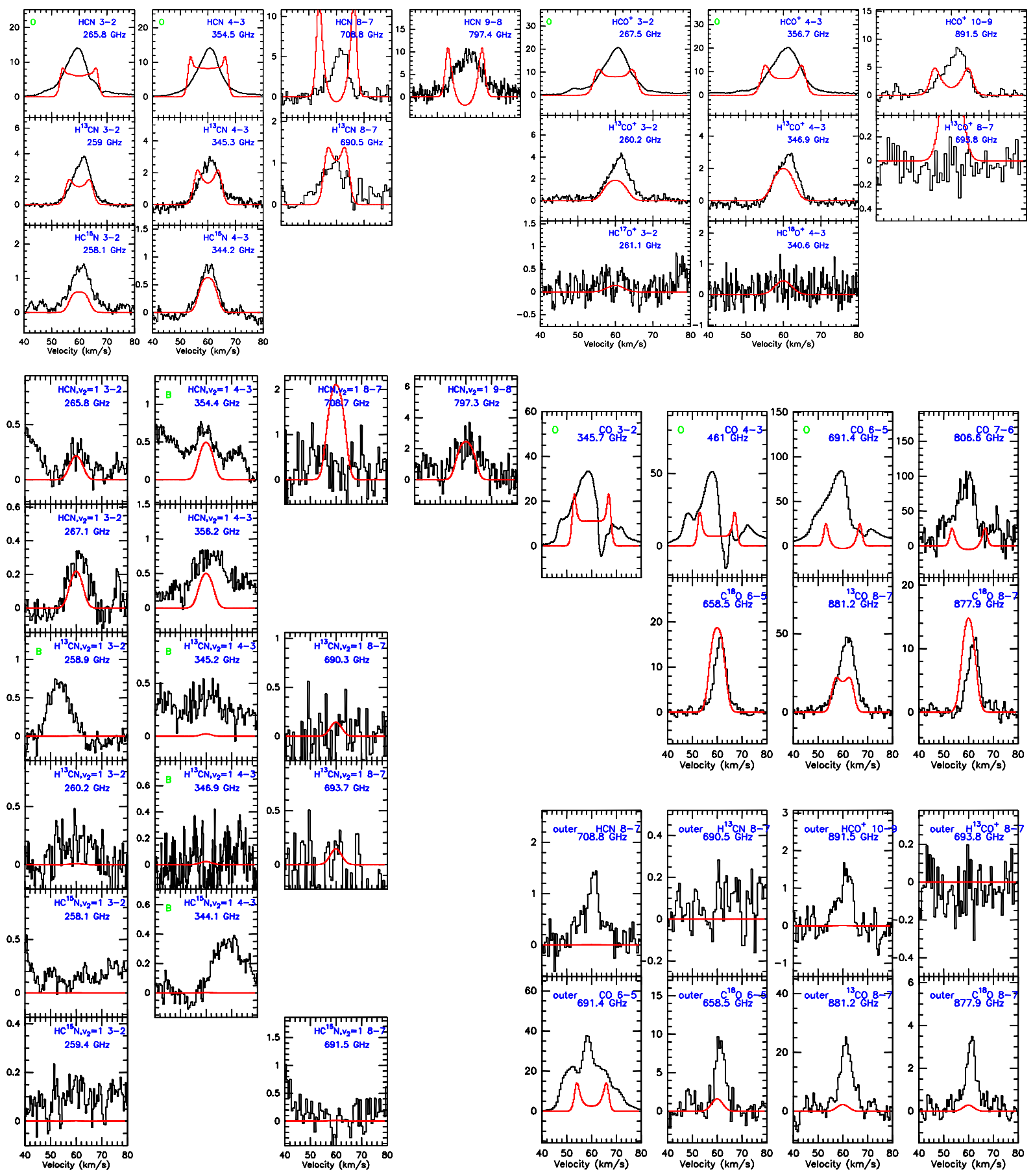

Figure 3.11: Lines towards W51d. The model from Table 3.8 is overlaid in red.

\subsubsection{W51e}

W51e contains two hot cores, separated by 7" and associated with the UCHII regions e2 and e8 (Zhang et al. 1998). Both regions exhibit asymmetric line profiles, which were interpreted by Rudolph et al. (1990) as signatures of overall gravitational collapse. However, Ho \& Young (1996) presented higher angular resolution observations and concluded that the collapse is localized to the two cores. Shi et al. (2010) shows that W51e2 has fragmented into additional pieces.

Figure 3.10 shows the observed lines and the best-fit model (Table 3.8). Both cores associated with e2 and e8 fall within our beam, which we had to ignore in the modeling. Our pointing was centered on the southern hot core (e1/e8). Infall is clearly seen by the strong blue asymmetries. 

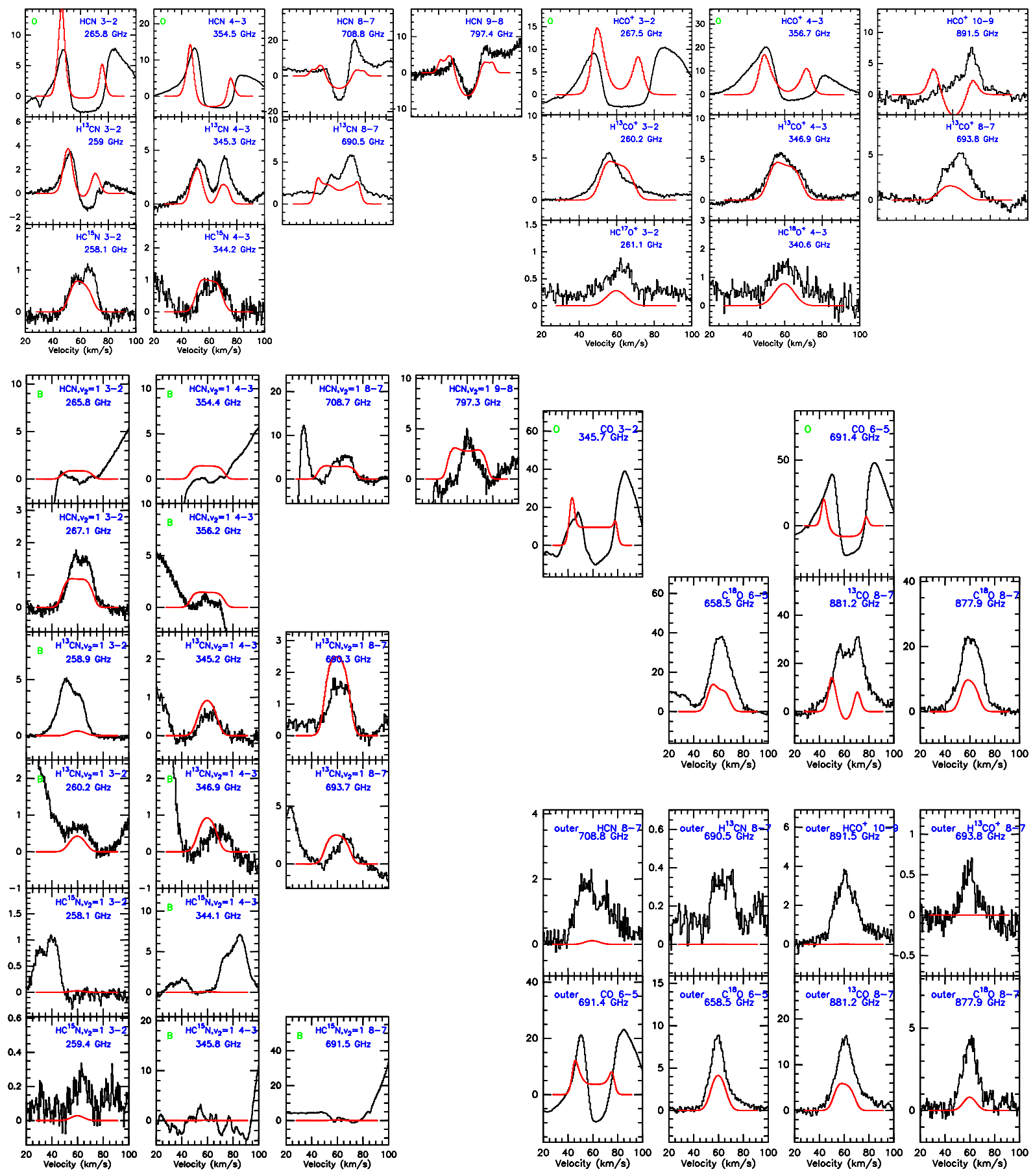

Figure 3.12: Lines towards SgrB2-M. The model from Table 3.8 is overlaid in red.

\subsubsection{W51d}

This hot core is located between the UCHII regions d1 and d2 (Zhang et al. 1998), and could host a rotating structure with outflow (Zapata et al. 2009a). Infall motion is traced by SMA observations of CN absorption (Zapata et al. 2008b).

Figure 3.11 shows the observed lines and the best-fit model (Table 3.8). The lack of self-absorption cannot be explained by spherical symmetry. Line emission in the outer pixels of CHAMP+ is much stronger than modeled.

\subsubsection{SgrB2(M)}

SgrB2 Main contains a large number of UCHII regions (Gaume \& Claussen 1990), and molecular gas of very high temperature is present (e.g. Wilson et al. 2006; Comito et al. 2003). The envelope was modeled by Lis \& Goldsmith (1990). 

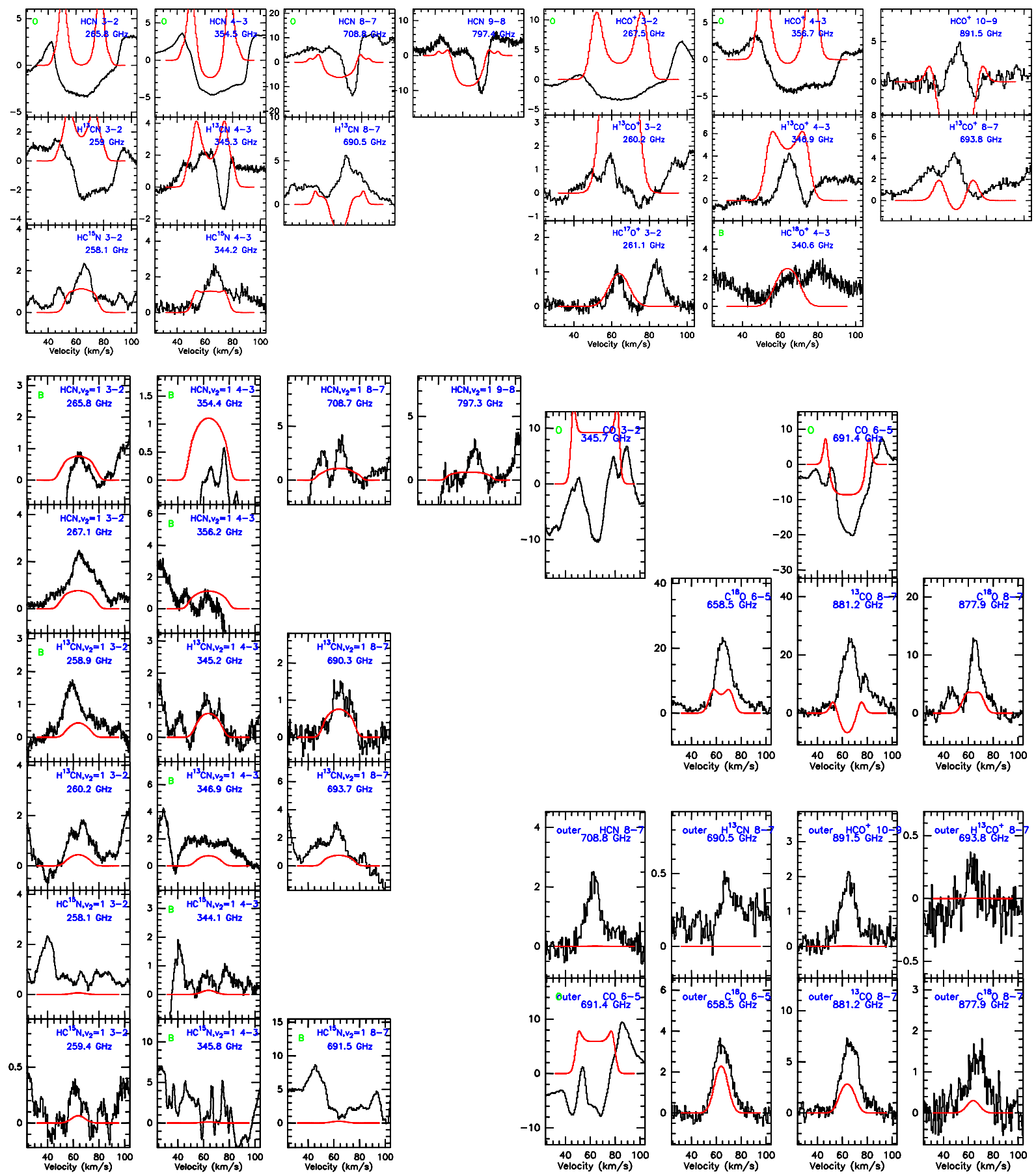

Figure 3.13: Lines towards SgrB2-N. The model from Table 3.8 is overlaid in red.

Figure 3.12 shows the observed lines and the best-fit model (Table 3.8). Warm and dense clumps can be seen by the $\mathrm{HCN}$ and $\mathrm{HCO}^{+}$emission in the outer pixels, which is not included in our model. Vibrationally excited $\mathrm{H}^{13} \mathrm{CN}$ is detected. Although the model incorporates infall, the asymmetry in the high-J lines points to an expanding velocity field in the inner part. We note that Herschel/HIFI data and a model that takes this into account are published in a separate paper (Rolffs et al. 2010).

\subsubsection{SgrB2(N)}

$\mathrm{SgrB} 2$ North is another very massive hot core 50" north of SgrB2(M), with a rich organic chemistry (e.g. Belloche et al. 2008). The hot and dense molecular gas is concentrated around the hypercompact HII region K2 (Liu \& Snyder 1999). de Vicente et al. (2000) showed with observations of vibrationally excited $\mathrm{HC}_{3} \mathrm{~N}$ that hot cores are also present between $\mathrm{M}$ and $\mathrm{N}$. 
Figure 3.13 shows the observed lines and the best-fit model (Table 3.8). The strong and broad lines are not clearly reproduced by the model. Very broad absorption in the low-J lines are accompanied by redshifted absorption in the high-J lines, pointing to non-spherical infall. Vibrationally excited $\mathrm{H}^{13} \mathrm{CN}$ is present, and the outer pixels again show line emission (e.g. $\mathrm{H}^{13} \mathrm{CN} 8-7$ ) that require high densities and temperatures at these locations, and hence clumpiness.

\subsection{Discussion}

The limitations of the data (too coarse angular resolution) and the radiative transfer modeling do not allow a reliable determination of the structure of the sources. Some observational features cannot be reproduced by any of the models, and the fits can be successfully performed by a variety of models. However, by systematically comparing models to the data, we can place some constraints on the source structure.

\subsubsection{Heating engine}

The high luminosities and submm fluxes imply that a massive (proto)star(s) is the heating engine. The lines from vibrationally excited HCN clearly show the high temperatures and the large heated mass. The self-absorption features prove the existence of a temperature gradient with lower temperatures closer to us. The observations are consistent with there being internal heating of the cores.

Although we model the sources with only one central star, we cannot exclude multiple heating sources. Multiplicity could indeed partly explain the deviations between model and data in many sources, and it is often observed by interferometers. This is unsurprising, because the sources are clusters in the making.

\subsubsection{Density}

Hot cores are basically heated density concentrations. For a power-law density distribution, an exponent of 1.5 seems consistent with the data, with a general tendency toward slightly larger values. The actual density depends on the dust opacity. For the opacity bare,e5 (Ossenkopf \& Henning 1994) and a typical model, densities at the dust sublimation radius (a few hundred AU from the star) reach values as high as $10^{9} \mathrm{~cm}^{-3}$ and fall off to $10^{3}$ or $10^{4}$ $\mathrm{cm}^{-3}$ at a radius of a pc, where the dust has cooled to the ambient temperature. Typical central column densities are $10^{25} \mathrm{~cm}^{-2}$.

Interferometer observations often reveal the presence of clumps, or in general a different small-scale density distribution than the strongly peaked one of our models. On larger scales, the rather strong lines in the outer pixels of CHAMP+ and the relatively little or no self-absorption in many sources are also indicative of clumpiness. The line emission in the CHAMP+ outer pixels clearly implies that there are higher densities at these locations. If that were the case for spherical (or cylindrical) symmetry, the continuum would be much higher than observed. A solution is the presence of high-density clumps, which provide the line emission but a moderate continuum due to beam dilution. The sources are likely to be embedded in a larger structure (see e.g. Myers 2009).

\subsubsection{Velocity field}

Infall motions are imprinted in the line shapes, as seen by blue asymmetries (lower velocities stronger). Freely contracting spheres (i.e., an inward velocity set by the mass inside) can be excluded, as in this case the lines would be far more asymmetric than observed. A tighter fit is obtained by reducing this free-fall speed to $10 \%$ in four sources (ngc, b2m, g10, g31) or 20\% in two sources (w51e, g34). This corresponds to infall velocities of around $1 \mathrm{~km} / \mathrm{s}$. We note that such a velocity field cannot be in a steady state, since the accretion rate decreases inwards. At the outer radius, these infall velocities correspond to cluster mass accretion rates of $10^{-3}$ to $10^{-1}$ solar masses per year. Dividing these rates by the source mass, each solar mass (representing a potential future star) would receive roughly $10^{-6}$ solar masses per year, and the timescale is thus $10^{6}$ years. Since in the remaining six sources there are also symmetric or red asymmetric line profiles, a pure infall does not reproduce the lines well and we model them for now with zero velocity. The red asymmetric lines point to the presence of expansion motions, and we investigate this more complicated velocity field in a separate paper, based on Herschel/HIFI data (Rolffs et al. 2010).

In addition, we see high-velocity wings in many lines of $\mathrm{CO}, \mathrm{HCN}$, and $\mathrm{HCO}^{+}$, resulting from outflows (which would also destroy any spherical symmetry). The line width represents internal motions, which are several $\mathrm{km} / \mathrm{s}$. The lowest amount of turbulence is found in g327, and the highest in the Galactic center sources b2n and b2m. 


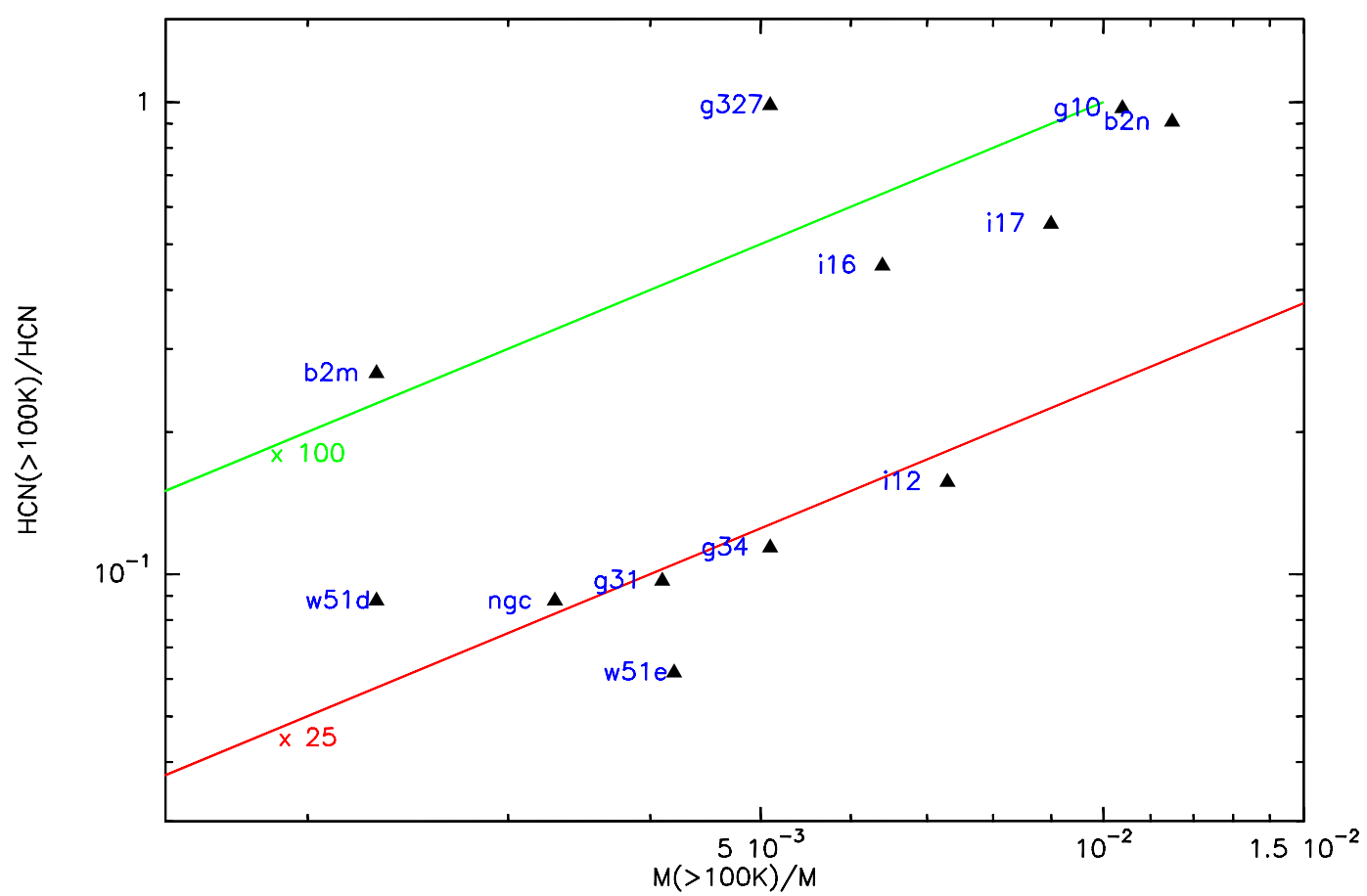

Figure 3.14: Mass fraction of hot gas vs. mass fraction of hot HCN in the best-fit models. Two groups of sources can be distinguished, one with detections of vibrationally excited $\mathrm{H}^{13} \mathrm{CN}$, having an $\mathrm{HCN}$ abundance enhancement at high temperatures of around 100 and on average a higher fraction of hot gas, and one with an HCN abundance enhancement of around 25, coming from freeze-out of $\mathrm{HCN}$ at low temperatures.

\subsubsection{Molecular abundances}

For the densities corresponding to the dust opacity bare,e5, we find typical abundances relative to $\mathrm{H}_{2}$ of $10^{-7}$ $(\mathrm{HCN}), 10^{-8}\left(\mathrm{HCO}^{+}\right)$, and $10^{-4}(\mathrm{CO})$. While we allow the $\mathrm{CO}$ abundance to be exactly $10^{-4}$ everywhere, the mentioned abundances of $\mathrm{HCN}$ and $\mathrm{HCO}^{+}$are only an order of magnitude between 50 and $100 \mathrm{~K}$. On the basis of Garrod et al. (2008), we allow a reduced abundance of these molecules below 40 or $50 \mathrm{~K}$ (due to molecular depletion), and this is actually needed to reproduce the observed self-absorptions. An evaporation temperature of $100 \mathrm{~K}$, together with water, would not match most of the observed lines.

In sources where vibrationally excited $\mathrm{H}^{13} \mathrm{CN}$ is detected, $\mathrm{HCN}$ must be very abundant at high temperatures $\left(10^{-6}\right.$ to several $\left.10^{-5}\right)$, which is consistent with predictions from chemical models (Rodgers \& Charnley 2001) and also needed by Boonman et al. (2001). However, the astrochemical reason for this very high abundance remains unclear. Figure 3.14 shows the abundance enhancement in the models, allowing us to identify the two groups with and without vibrationally excited $\mathrm{H}^{13} \mathrm{CN}$. The former group has on average not only a larger fraction of hot gas, but also a higher $\mathrm{HCN}$ abundance in this gas.

In contrast, no vibrationally excited $\mathrm{HCO}^{+}$is detected. Since it has a very similar level structure and line strengths as $\mathrm{HCN}$, this non-detection means that it must be much less abundant than $\mathrm{HCN}$ at high temperatures. Relative to the $\mathrm{HCN}$ (and $\mathrm{H}^{13} \mathrm{CN}$ ) abundances and intensities of the vibrational lines, the $\mathrm{HCO}^{+}$abundance cannot be higher than about $10^{-8}$ above $100 \mathrm{~K}$. We allow a reduced abundance of $\mathrm{HCO}^{+}$at high temperatures (van der Tak \& van Dishoeck 2000), but our data are of insufficient quality to constrain this.

\subsubsection{Limitations of our modeling approach}

We first note that we are limited to spherical symmetry. However, two- or three-dimensional modeling would not be reliably constrained by our data; the lines need to be spatially well resolved to achieve this. Consequently, we chose to consider only centrally heated models. The density was assumed to follow a power law. While this strong central condensation is consistent with the framework of central heating, a more realistic picture of a forming star cluster would include multiple heating sources and likely a central flattening of the density. These possibilities are not covered by our modeling, nor is a clumpy, geometrically flattened or filamentary structure. In addition, outflows cannot be considered in spherically symmetric models. 
The grid that we use for the continuum-relevant parameters is complete enough, but the selection of models relies on the LABOCA data, which do not have sufficient angular resolution. Owing to limited computational power, we could not test all selected models, and only a few chemical structures, thus we were unable to find the optimal global combination of the parameters. In addition, the chemical structures are crude approximations of the abundance variations, and are not computed from chemical models. The dust opacity is assumed to be constant throughout the source, although it is likely to vary (e.g. due to evaporation of ice mantles).

\subsection{Conclusions}

We have observed a sample of 12 hot cores in lines of $\mathrm{HCN}, \mathrm{HCO}^{+}$, and $\mathrm{CO}$ with the APEX telescope. With the aim of reproducing the line shapes, we tested spherical models with density power-law gradients and central heating. Our results can be summarized as follows:

1. Vibrational excitation: Vibrationally excited HCN (1000 K above ground) is very prominent in our sources, and even vibrationally excited $\mathrm{H}^{13} \mathrm{CN}$ is clearly detected in half of them $(\mathrm{g} 327, \mathrm{i} 16, \mathrm{i} 17, \mathrm{~b} 2 \mathrm{n}, \mathrm{b} 2 \mathrm{~m}, \mathrm{~g} 10)$. This result is surprising because it means that vibrationally excited $\mathrm{HCN}$ is optically thick and that there is much more hot gas than previously thought. The lines are still visible at $800 \mathrm{GHz}$, meaning that dust does not obscure the hot gas at these frequencies. They can be well modeled; to reproduce vibrationally excited $\mathrm{H}^{13} \mathrm{CN}$, an enhanced $\mathrm{HCN}$ abundance at high temperatures is necessary. On the other hand, no vibrationally excited $\mathrm{HCO}^{+}$is detected. Given its very similar molecular structure, this means it is much less abundant than $\mathrm{HCN}$ under hot, dense conditions.

2. Self-absorption: In most sources, the main transitions are heavily self-absorbed. This finding, together with the corresponding lines from more rare isotopologues, is qualitatively reproduced by our models. It is caused by very high optical depths and the temperature gradient, and depends also on the abundance structure (e.g. freeze-out). However, well-peaked, but highly optically thick lines, similar to those found in w51d and i12, cannot be modeled in our framework, and must be produced by geometry effects.

3. Outer pixels: The high-frequency CHAMP+ receiver on APEX has seven pixels. We were mainly interested in the central pixel, but also modeled the average of the other pixels, which are about two beam sizes $\left(15^{\prime \prime}-\right.$ $20^{\prime \prime}$ ) from the center. The modeling always leads to much lower intensities in the outer pixels than observed. We conclude that the warm, dense gas is not as highly concentrated as predicted by our models, which have steep radial gradients in temperature and density. Clumpiness and multiple heating sources are present instead, as expected for a cluster environment.

4. Velocity field Information about the velocity field is encoded in the line shapes, in particular their asymmetries. The blue side (lower velocities) is in most cases stronger than the red side, which we model as an inward velocity. The free-fall speed, which would be unhampered contraction, must be reduced to 10 or $20 \%$ to reproduce the line shapes. This means that there is infall, but also mechanisms to slow it down. However, we have modeled half of our sources as static, since the velocity field is often more complicated, shown by outflow wings in $\mathrm{CO}$, symmetric lines, and red asymmetries. This means that expansion motions are also present in some sources, e.g. b2m and g327, caused by the onset of feedback from massive stars (see the paper on Herschel/HIFI data of b2m, Rolffs et al. 2010).

5. Basic assumptions For the radiative transfer modeling, we are limited to a very simplified source structure. In particular, we assume spherical symmetry with a radial power-law gradient in density and pure central heating, which provides a rough approximation of the temperature. Molecular abundances are not computed from chemical models, but variations are approximated as jumps at certain temperatures. While these crude simplifications are able to explain many observational features, the assumptions are too simple to reproduce everything. Three-dimensional radiative transfer, coupled to physical and chemical models and based on high-resolution interferometric data, is needed to reliably determine the structure of hot cores.

\section{Acknowledgements}

We thank Floris van der Tak for the molecular data of vibrationally excited HCN and for the support with RATRAN. R.Rolffs acknowledges support by the International Max Planck Research School for Astronomy and Astrophysics. We thank Claudia Comito for help in the acquisition of the first part of the data. 


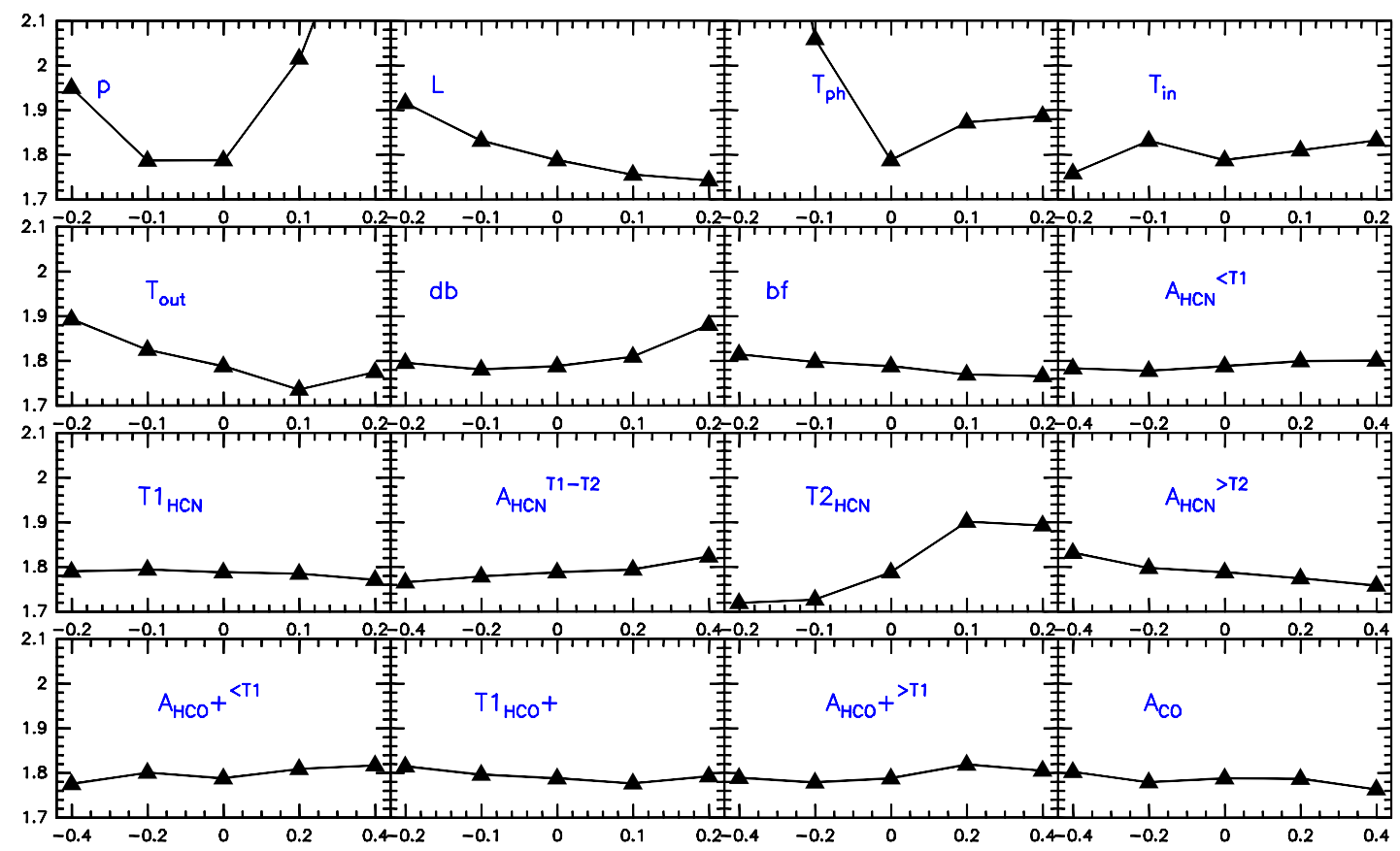

Figure 3.15: $\chi^{2}$ for the variation of each parameter in the model for G10.47+0.03. The abundances were varied by 20 and $40 \%$, the other parameters by 10 and $20 \%$.

\subsection{Additional Information}

To get a handle on the uncertainties of the parameters, each parameter is varied while holding the other parameters fixed. As an example, the best-fit model for G10.47+0.03 is taken. The parameters are those shown in Table 3.8, plus $T_{\text {in }}(1500 \mathrm{~K})$ and $T_{\text {out }}(20 \mathrm{~K})$. The variation is by \pm 10 and $20 \%$ (for the abundances by \pm 20 and $40 \%$ ). Figure 3.15 shows the $\chi^{2}$ of these parameter variations. However, a model with lower $\chi^{2}$ is not necessarily one that would be judged as better by eye. For instance, one would give more weight to specific lines, or to the similarity of the line shapes.

Part of the problem is that the models do not attempt to reproduce all facets of the source, e.g. it is impossible to properly treat outflows in a spherical model. The observed spectra do show these features though, which is why unweighted $\chi^{2}$ values are not a good figure of merit.

The density power-law index $p$ could also be a bit lower than 1.75 , while the luminosity tends to higher values than $5.6 \times 10^{5} \mathrm{~L}_{\odot}$. Especially lower values of the photospheric temperature of $65 \mathrm{~K}$ make the fit worse. Variations of the inner temperature does not have a huge effect, and the outer temperature could also be a bit higher than $20 \mathrm{~K}$. The linewidth has a broad minimum at the adopted value of $5 \mathrm{~km} / \mathrm{s}$ FWHM. The velocity slightly tends to a stronger infall, supporting the infall interpretation. Abundances and jump temperatures are generally not well constrained; only the temperature at which the $\mathrm{HCN}$ abundance jumps to its high value of $3 \times 10^{-5}$ could be even lower than $100 \mathrm{~K}$. 


\section{Chapter 4}

\section{Herschel paper: Reversal of infall in SgrB2(M) revealed by Herschel/HIFI observations of $\mathrm{HCN}$ lines at $\mathrm{THz}$ frequencies}

Sections 4.2 to 4.7 appeared as a letter in the Astronomy $\mathcal{E}$ Astrophysics special issue for first results from Herschel/HIFI: Rolffs, R., Schilke, P., Comito, C., Bergin, E. A., van der Tak, F. F. S., Lis, D. C., Qin, S.-L., Menten, K. M., Güsten, R. et al. 2010, AEAA, 521, L46+ (Rolffs et al. 2010, received 31 May 2010 / accepted 7 July 2010)

\subsection{Overview}

Telescope: The Herschel Space Observatory is a space telescope of $3.5 \mathrm{~m}$ diameter, launched in May 2009. Besides two imaging instruments, it hosts the spectral line receiver HIFI (Heterodyne Instrument for the Far Infrared), which covers the 490-1250 GHz and 1410-1910 GHz regions, separated in 7 bands. Herschel is a mission of the European Space Agency (ESA).

Observations: The Herschel key project HEXOS (Herschel/HIFI Observations of EXtraOrdinary Sources) consists of full line surveys of five sources (Bergin et al. 2010). Since Peter Schilke and Claudia Comito are members of the HEXOS team, I have obtained access to the SgrB2-M data. Analysis of the HCN lines from the first part of these data (bands 1a, 1b, 2a, 4b) was published in Rolffs et al. (2010, next sections). Additional frequencies and lines from $\mathrm{HNC}$ and $\mathrm{HCO}^{+}$are presented in Sect. 4.8.

Modeling: On the basis of previous modeling with RATRAN that I have performed for the APEX data (Chapter 3), I was able to first construct a physical structure that fits the HIFI baseline levels. Second, HCN abundance and velocity field were adapted to fit the HCN lines. The models are spherically symmetric, with power-law density gradient and central heating.

Results: The asymmetries of the self-absorbed HCN lines change systematically with $J$ : From a blue profile at low $J$ to a red profile at high $J$. This could be reproduced by a model whose velocity field changes from infall in the outer parts to expansion in the inner parts. This finding suggests that the gravitational infall is reversed close to the center of the forming cluster, owing to feedback from the massive stars.

\subsection{Abstract}

Aims: To investigate the accretion and feedback processes in massive star formation, we analyze the shapes of emission lines from hot molecular cores, whose asymmetries trace infall and expansion motions. 
Methods: The high-mass star forming region SgrB2(M) was observed with Herschel $^{1} / \mathrm{HIFI}$ (HEXOS key project) in various lines of HCN and its isotopologues, complemented by APEX data. The observations are compared to spherically symmetric, centrally heated models with density power-law gradient and different velocity fields (infall or infall+expansion), using the radiative transfer code RATRAN.

Results: The HCN line profiles are asymmetric, with the emission peak shifting from blue to red with increasing $\mathrm{J}$ and decreasing line opacity $\left(\mathrm{HCN}\right.$ to $\mathrm{H}^{13} \mathrm{CN}$ ). This is most evident in the HCN 12-11 line at $1062 \mathrm{GHz}$. These line shapes are reproduced by a model whose velocity field changes from infall in the outer part to expansion in the inner part.

Conclusions: The qualitative reproduction of the HCN lines suggests that infall dominates in the colder, outer regions, but expansion dominates in the warmer, inner regions. We are thus witnessing the onset of feedback in massive star formation, starting to reverse the infall and finally disrupting the whole molecular cloud. To obtain our result, the THz lines uniquely covered by HIFI were critically important.

\subsection{Introduction}

Massive stars form deep inside molecular clouds. While this obscures the process at infrared or shorter wavelengths, it offers the possibility of investigating high-mass star formation using dust emission and molecular lines from the radio to the far-infrared. The shapes of molecular lines are a particularly powerful tool, since they trace the density and temperature gradients as well as the velocity field. Even when the cores are not spatially resolved, radiative transfer modeling of a variety of lines can constrain their structure. The unique spectral coverage of HIFI opens up the full potential of this technique, since both the excitation of rotational transitions and the dust opacity increase with frequency. While the former allows us to study higher temperatures and densities, the latter leads to stronger continuum radiation, thereby facilitating the production of absorption features.

A particularly important stage of high-mass star formation is the hot core phase. When massive stars begin burning hydrogen, they are still accreting and surrounded by dense molecular gas, which is heated and emits a rich spectrum of molecular lines. Various feedback mechanisms (radiation pressure, pressure increase due to ionization, stellar winds, bipolar outflows) push the gas outwards. Finally, this dominates over gravitational accretion, and the molecular cloud is disrupted.

The giant molecular cloud Sagittarius B2 is the most massive and active star-forming region in our Galaxy. It is located close to the Galactic center at a distance of $7.8 \mathrm{kpc}$ (Reid et al. 2009). SgrB2(Main) is a tight cluster of ultracompact HiI regions (Gaume \& Claussen 1990), associated with a massive hot molecular core. Its luminosity is estimated to be $6.3 \times 10^{6} \mathrm{~L}_{\odot}$ (Goldsmith et al. 1992), the most luminous hot core of the Galaxy. Both infall and a decelerating outflow were seen by Qin et al. (2008).

In this paper, we present new results for the velocity field of SgrB2(M), based on Herschel/HIFI observations of $\mathrm{HCN}$ and complemented by APEX data.

\subsection{Observations}

Herschel is a new space observatory with a $3.5 \mathrm{~m}$ antenna operating at submm to far-infrared wavelengths (Pilbratt et al. 2010). Its spectral line receiver is HIFI (Heterodyne Instrument for the Far Infrared, de Graauw et al. 2010). The guaranteed time key project HEXOS (Herschel/HIFI Observations of EXtraOrdinary Sources, Bergin et al. $2010)$ includes a full line survey of $\operatorname{SgrB} 2(\mathrm{M})\left(\alpha_{J 2000}=17^{h} 47^{m} 20.35^{s}\right.$ and $\left.\delta_{J 2000}=-28^{\circ} 23^{\prime} 03.0^{\prime \prime}\right)$. In March 2010, this was started with HIFI bands $1 \mathrm{a}, 1 \mathrm{~b}, 2 \mathrm{a}$, and 4b, providing coverage of the frequency ranges $479-$ $726 \mathrm{GHz}$ and $1051-1121 \mathrm{GHz}$. Each band contains one rotational transition from $\mathrm{HCN}, \mathrm{H}^{13} \mathrm{CN}$, and $\mathrm{HC}^{15} \mathrm{~N}$ (Fig. 4.1), and two rotational transitions within their first vibrational state, which lies around $1000 \mathrm{~K}$ higher in energy (Fig. 4.2). Transition frequencies of HCN (Thorwirth et al. 2003a; Fuchs et al. 2004) are taken from CDMS (Müller et al. 2001, 2005). HIFI spectral scans are carried out in dual beam switch (DBS) mode, where the DBS reference beams lie approximately $6^{\prime}$ apart. The wide band spectrometer (WBS) is used as a back-end, providing a spectral resolution of $1.1 \mathrm{MHz}$ over a 4-GHz-wide intermediate frequency (IF) band. To allow separation of the two sidebands, bands 1a, 1b, 2a, and 4b were scanned with a redundancy of 4, 8, 8, and 4, respectively (Comito $\&$ Schilke 2002). The data were calibrated using the standard pipeline released with version 2.9 of HIPE (Ott 2010), and subsequently exported to $\mathrm{CLASS}^{2}$ using the HiClass task within HIPE. Deconvolution of the DSB

\footnotetext{
${ }^{1}$ Herschel is an ESA space observatory with science instruments provided by European-led Principal Investigator consortia and with important participation from NASA.

${ }^{2}$ Continuum and Line Analysis Single-dish Software, distributed with the GILDAS software, see http://www.iram.fr/IRAMFR/GILDAS.
} 

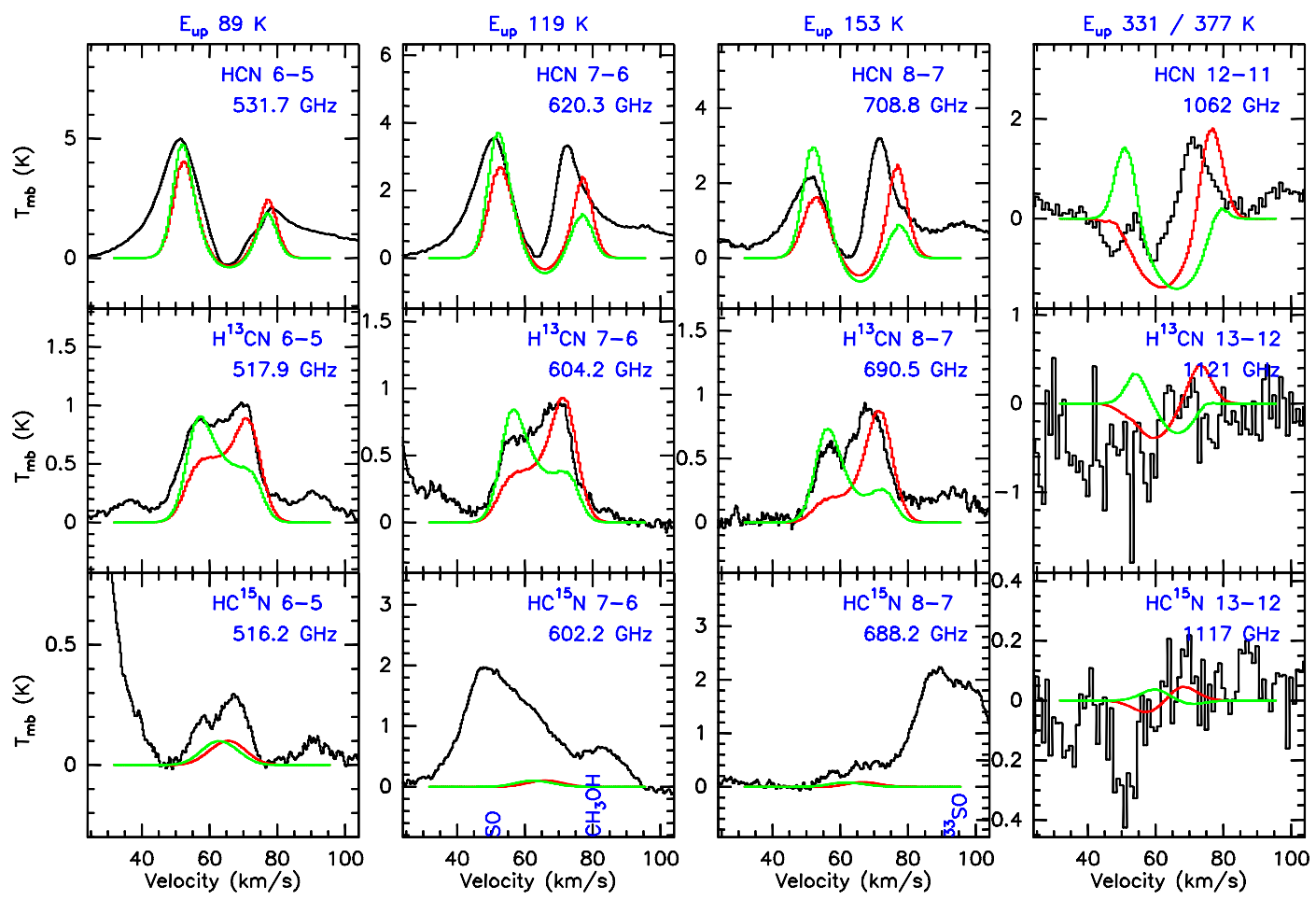

Figure 4.1: Herschel/HIFI observations of HCN in SgrB2(M). The green model has a constant infall, the red model has infall in the outer part and expansion in the inner region. Note the different asymmetries in the lines, which are reproduced by the red model. Upper state energies are stated above the plot.

data into single-sideband (SSB) format was performed using CLASS. All the HIFI data presented here, spectral features and continuum emission, are deconvolved SSB spectra. The horizontal and vertical polarizations differ by around $10 \%$, and were averaged. The intensity scale is main-beam temperature, and results from applying a beam efficiency correction of about 0.68 .

APEX (Atacama Pathfinder Experiment) is a 12-m telescope located at $5100 \mathrm{~m}$ altitude in the Atacama Desert, Chile, one of the optimal sites for submm observations on Earth (Güsten et al. 2006). The lines presented here (Fig. 4.3) are from a study of the structure of 12 hot cores (Rolffs et al. 2010, in prep.). Since the baseline levels in the ground-based data are not determined reliably enough to extract the continuum, the $850 \mu \mathrm{m}$ continuum is taken from ATLASGAL (Schuller et al. 2009).

\subsection{Modeling}

The spherical Monte Carlo radiative transfer code RATRAN (Hogerheijde \& van der Tak 2000) was employed to compute the molecular lines and the dust continuum. Using an iterative by-eye comparison, the fit to the data was obtained in two steps. First, the physical structure (density and temperature) was adapted to match the continuum. Second, the HCN abundance and the velocity were optimized for the lines.

The model we present here is centrally heated, its density following a radial power law with index 1.5. The dust opacity was taken from Ossenkopf \& Henning (1994), without either ice mantles or coagulation. At the inner radius, $1340 \mathrm{AU}$, the density is $1.8 \times 10^{8} \mathrm{~cm}^{-3}$ and the temperature is $1500 \mathrm{~K}$. The temperature was computed in an approximate way from central heating, using the diffusion equation in the inner part and balance between heating and cooling in the outer part, assuming an effective temperature for the dust photosphere of $58 \mathrm{~K}$. The temperature has a steeper gradient in the inner part, and falls off to $20 \mathrm{~K}$ at the outer radius of $5.6 \mathrm{pc}$, where the density is $7 \times 10^{3}$ $\mathrm{cm}^{-3}$. The model reproduces the continuum very well (Table 4.1). Its central dust optical depth is 0.4 at $345 \mathrm{GHz}$, 0.8 at $500 \mathrm{GHz}, 1.5$ at $700 \mathrm{GHz}$, and 3.7 at $1.1 \mathrm{THz}$.

To compute the lines, we used a turbulent 1/e half width of $7 \mathrm{~km} / \mathrm{s}(12 \mathrm{~km} / \mathrm{s} \mathrm{FWHM})$. The lines were additionally broadened by their high optical depth. We found no evidence of significant variations in the turbulent width with radius. The $\mathrm{HCN}$ abundance increases with temperature from $10^{-8}$ at temperatures below $100 \mathrm{~K}$, to $3 \times 10^{-8}$ between 100 and $300 \mathrm{~K}$, to $3 \times 10^{-7}$ at temperatures above $300 \mathrm{~K}$. The first jump is mainly needed to reproduce the intensities of the isotopologues and of vibrationally excited HCN, the second jump serves to fit vibrationally 


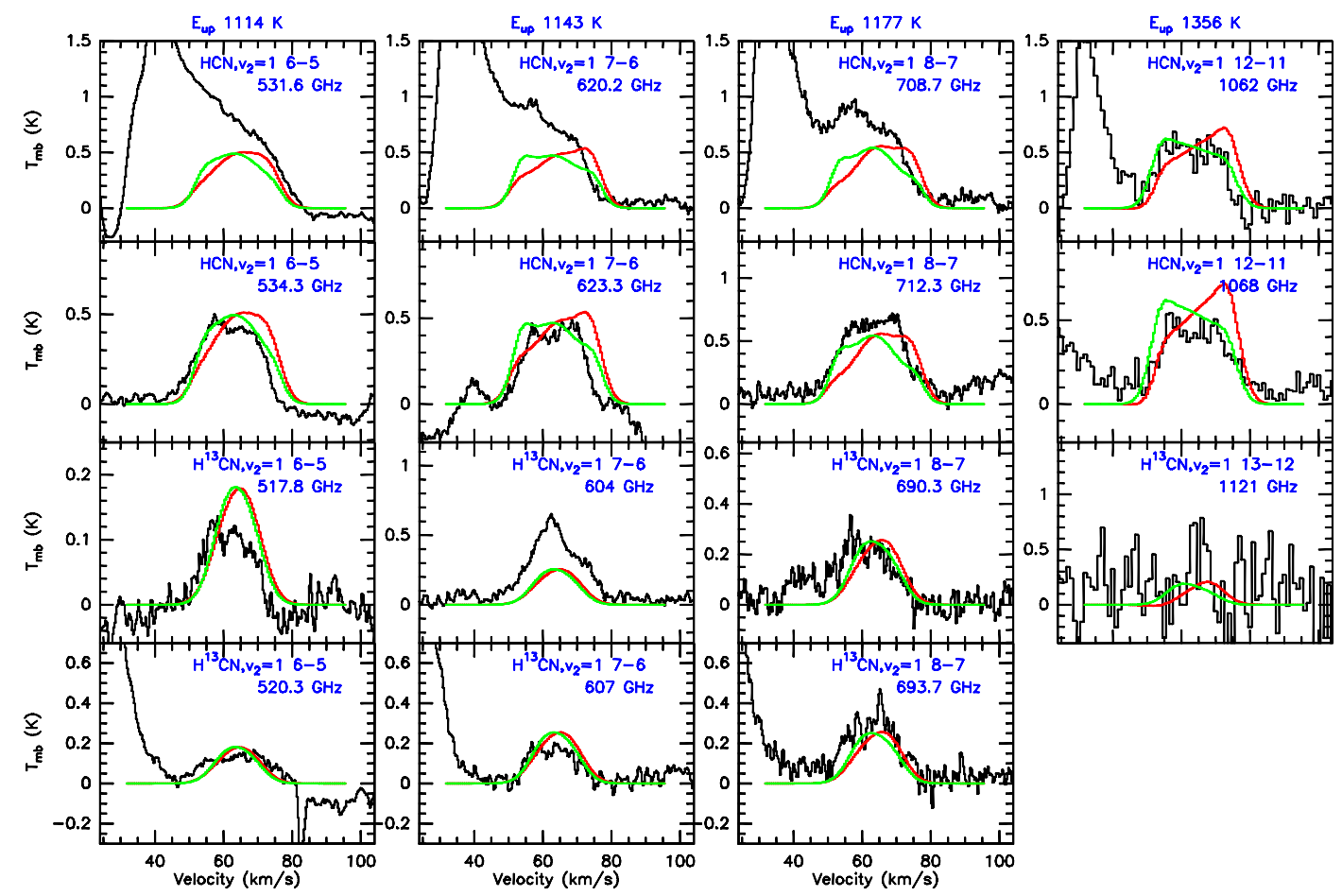

Figure 4.2: Herschel/HIFI observations of vibrationally excited HCN in SgrB2(M), overlaid with the pure-infall (green) and the expansion+infall model (red). The kinematics are not well constrained in this hot, innermost region.
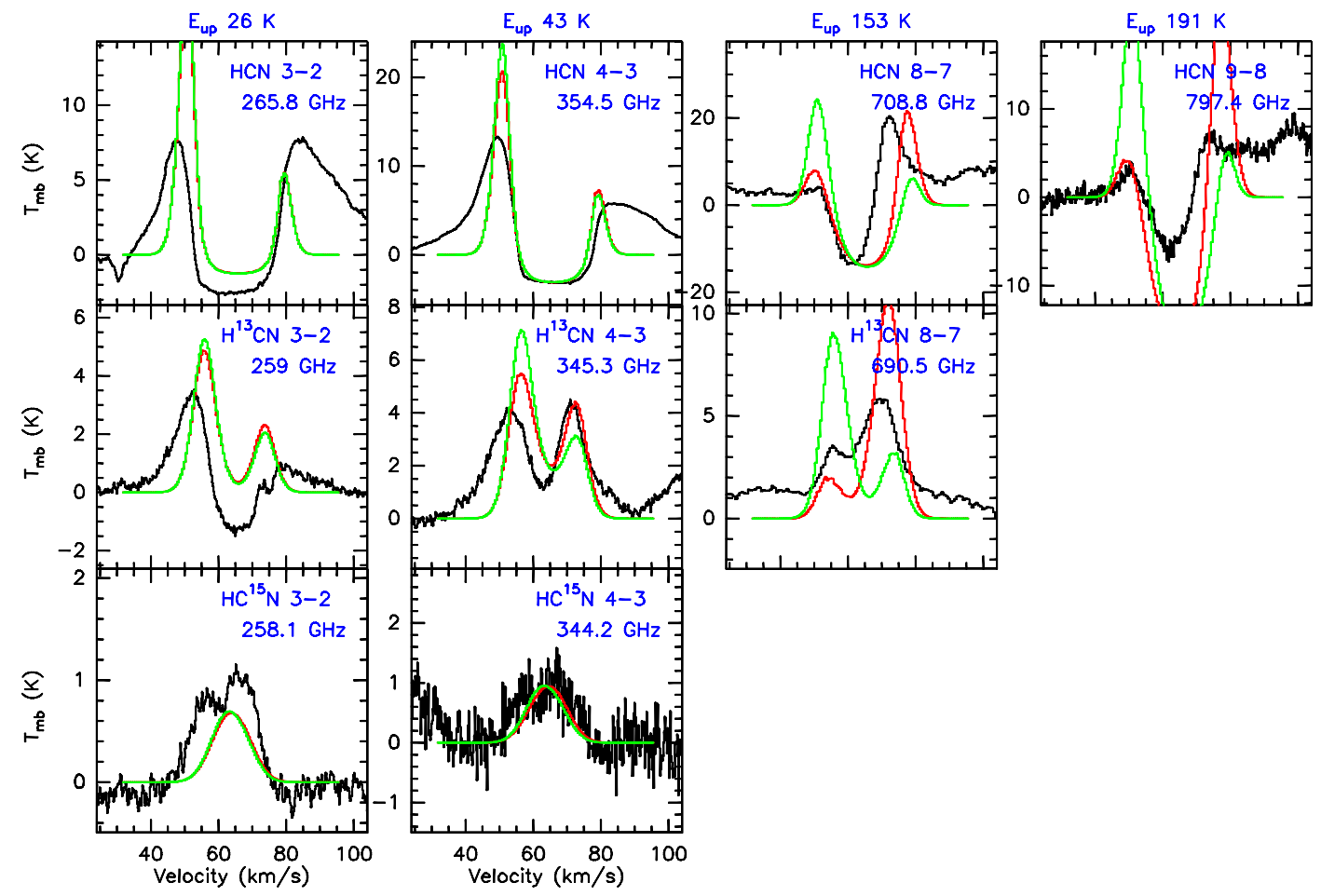

Figure 4.3: APEX observations of $\mathrm{HCN}$ in $\operatorname{SgrB} 2(\mathrm{M})$, overlaid with the pure-infall (green) and the expansion+infall model (red). Although less clear than in the HIFI data, the same asymmetry change can be seen. 


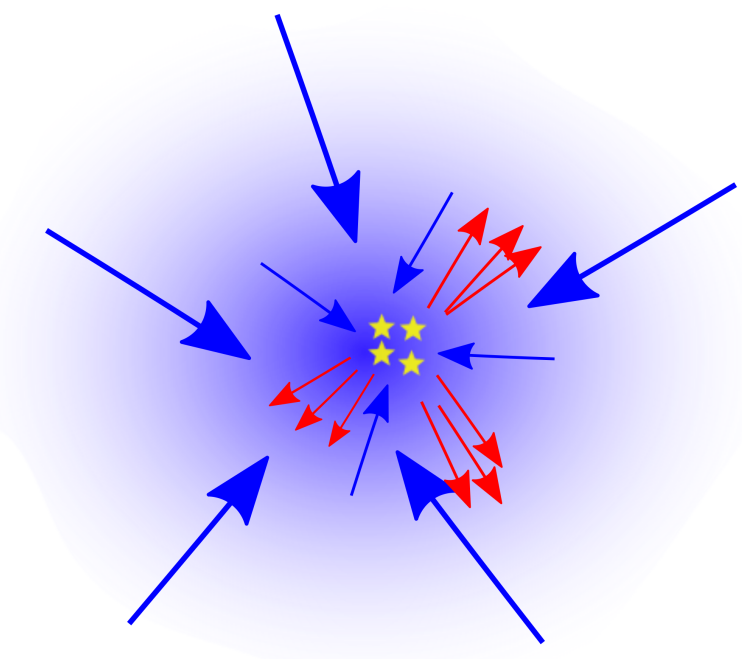

Figure 4.4: Sketch of the scenario described in the text. In the outer parts of the cloud, infall dominates. In the inner part, feedback has set in, in this example envisioned as multiple outflows, and gas is expelled from it. At the same time, infall continues along certain paths.

Table 4.1: Continuum fluxes of SgrB2(M) and the model.

\begin{tabular}{|lccc|}
\hline \hline $\begin{array}{c}\text { Frequency } \\
{[\mathrm{GHz}]}\end{array}$ & $\begin{array}{c}\text { Beam size } \\
{["]}\end{array}$ & $\begin{array}{c}\text { SgrB2(M) } \\
{[\mathrm{K}]}\end{array}$ & $\begin{array}{c}\text { model } \\
{[\mathrm{K}]}\end{array}$ \\
\hline 345 & 18.2 & 3.8 & 3.5 \\
\hline 500 & 43.1 & 2.4 & 2.4 \\
600 & 35.9 & 3.8 & 3.8 \\
700 & 30.8 & 5.7 & 5.7 \\
1100 & 19.6 & 14.6 & 14.2 \\
\hline
\end{tabular}

Notes. The first row is the $850 \mu \mathrm{m}$ flux from LABOCA on APEX, which contains also lines. The next rows are the baseline levels at the given frequencies in HIFI bands $1 \mathrm{a}, 1 \mathrm{~b}, 2 \mathrm{a}$ and $4 \mathrm{~b}$. The baseline level of HIFI is a reliable continuum measurement, since it is as strong as saturated absorption lines and compares well with ground-based continuum data.

excited $\mathrm{H}^{13} \mathrm{CN}$. These jumps could be due to evaporation of ice mantles and/or increased chemical production. We used a ${ }^{12} \mathrm{C} /{ }^{13} \mathrm{C}$ ratio of 20 and ${ }^{14} \mathrm{~N} /{ }^{15} \mathrm{~N}$ of 600 (Wilson \& Rood 1994). The green model has a constant infall velocity of $2 \mathrm{~km} / \mathrm{s}$, and the red model has the same velocity in its outer parts, but an expansion in its inner parts. Figure 4.5 shows the velocity field. We note that the exact shape of this, particularly the transition from infall to outflow, is not constrained by the observations, hence modeling the true velocity field by adopting this spherical approximation is an oversimplification (see Fig. 4.4).

A comparison with the observations (Figs. 4.1, 4.2, and 4.3) shows that the red model reproduces more closely the observed features. In particular, to reproduce the changes in the asymmetry that occur both from lower to higher $\mathrm{J}$ and from higher to lower optical depth (from $\mathrm{HCN} 6-5$ to $\mathrm{H}^{13} \mathrm{CN} 6-5$ ), a change in the velocity field is required, from infall in the colder, outer parts to expansion in the warmer, inner parts.

\subsection{Discussion}

We now discuss the velocity field and how it is physically determined.

In a gravitationally contracting sphere with density power-law index $p$, a first guess and upper limit to the infall speed would correspond to free fall, i.e., $\mathrm{v}_{\mathrm{ff}}(R)=\sqrt{\frac{2 G M(R)}{R}}$, where the mass inside the radius $R$ is $M(R) \propto R^{3-p}$ (for $p<3$ ), such that $\mathrm{v}_{\mathrm{ff}} \propto R^{\frac{2-p}{2}}$. In our model, this free-fall speed is roughly (at a radius of $1 \mathrm{pc}$ ) 10 times higher than the $2 \mathrm{~km} / \mathrm{s}$ of the model. Such high infall velocities can be excluded because they would produce lines that are far more asymmetric than observed.

The mass accretion rate $\dot{M}=\mathrm{v}_{\mathrm{acc}}(R) \frac{\mathrm{d} M(R)}{\mathrm{d} R}$, so $\mathrm{v}_{\text {acc }} \propto R^{p-2}$ for a constant mass accretion rate. Hence, infall accelerates in a spherical steady-state model with $p=1.5$. However, an accelerating infall, at least in the inner part, is inconsistent with the data. For simplicity, we chose a constant infall velocity, corresponding to accretion 


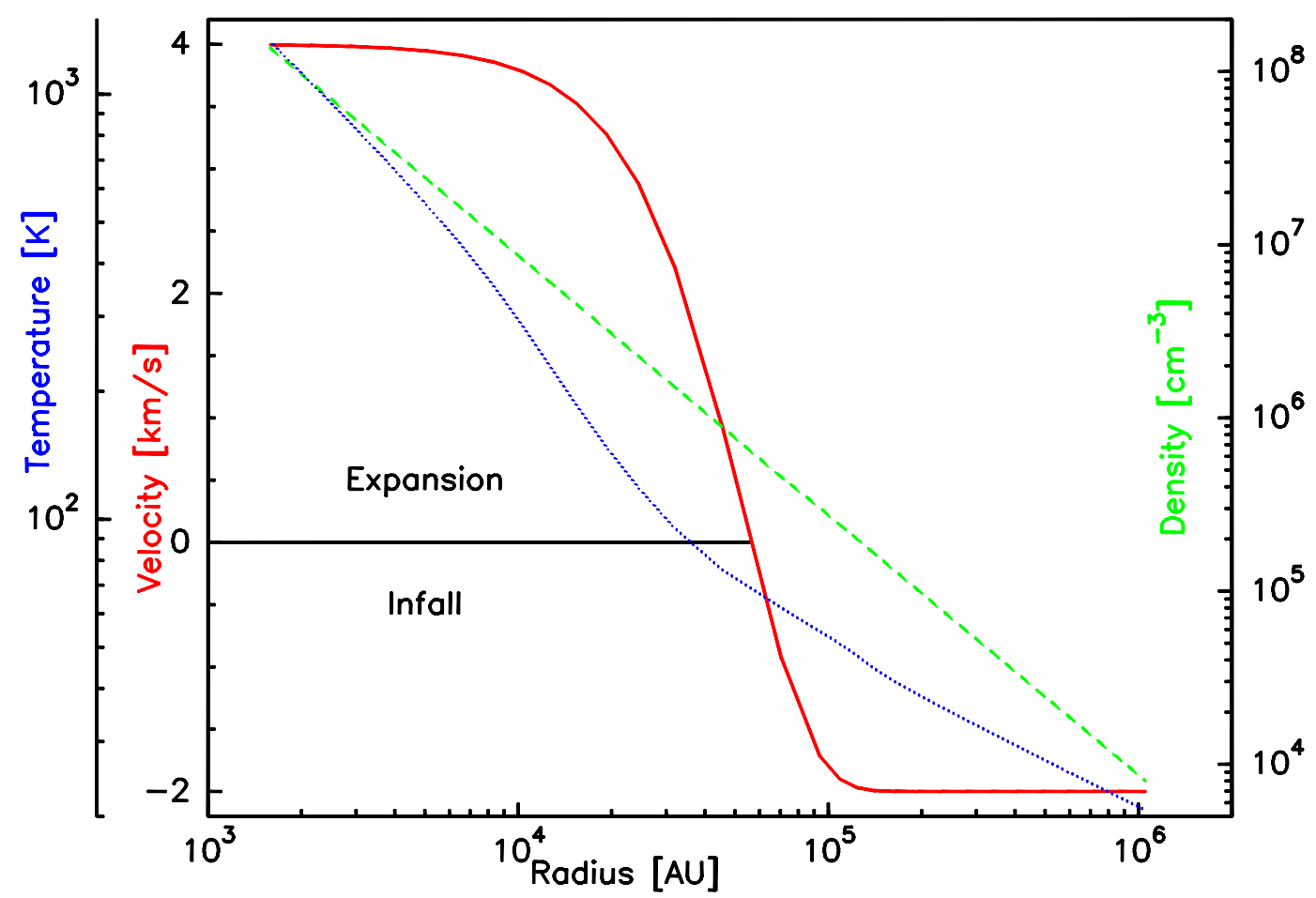

Figure 4.5: Physical structure of the model. Density is plotted as dashed green, temperature as dotted blue. The velocity field of the infall+expansion model is shown as the solid red curve (the infall model has a constant velocity of $-2 \mathrm{~km} / \mathrm{s})$. At the transition between infall and expansion, the temperature is $65 \mathrm{~K}$ and the density is $6 \times 10^{5} \mathrm{~cm}^{-3}$.

rates onto the cluster of $3 \times 10^{-1} \mathrm{M}_{\odot} / \mathrm{yr}$ at the outer boundary and $10^{-1} \mathrm{M}_{\odot} / \mathrm{yr}$ at a radius of $10^{5} \mathrm{AU}$. The excretion rate reaches $8 \times 10^{-2} \mathrm{M}_{\odot} / \mathrm{yr}$ at $2 \times 10^{4} \mathrm{AU}$.

The real velocity field must be non-spherical, and infall could well continue in the inner part (see Fig. 4.4 and also Qin et al. (2008)). The peak on top of the HCN 12-11 absorption (Fig. 4.1) could be a hint of that infall. Since the $\mathrm{H}^{13} \mathrm{CN}$ transitions are less asymmetric than modeled points in that direction, one may suspect that a linear combination of the pure infall and expanding models would produce the line spectra very well. For the HCN lines, because of their high optical depths, such a linear combination of intensities does not naturally reproduce the observed spectra; more sophisticated modeling, in which we abandon the spherical approximation, would be required. For overlying model and data (Figs. 4.1, 4.2, and 4.3), we assumed a source velocity of $64 \mathrm{~km} / \mathrm{s}$, although $60 \mathrm{~km} / \mathrm{s}$ would reproduce more closely the high-J lines. This blue shift of absorption towards higher $\mathrm{J}$ is consistent with our picture of a velocity field changing from infall to expansion, and is indicative of a deviation from spherical symmetry.

While gravity is clearly responsible for the infall, there must also be feedback mechanisms from newly formed stars working against gravity, thus regulating star formation. The velocity of the infalling envelope must be lower than that of pure gravitational collapse by around 90\%. Apart from magnetic braking, turbulence induced by bipolar outflows may be such a long-range feedback mechanism providing the necessary pressure (Wang et al. 2010). The massive stars in the center dominate the luminosity, and drive the expansion by means of stellar winds, radiation pressure (including reprocessed light), and heating and ionization of the gas, in particular thermal pressure of their HII regions (Krumholz \& Matzner 2009). Since in our model only $1 \%$ of the total mass of $7 \times 10^{5} \mathrm{M}_{\odot}$ is expanding, the outward momentum remains much lower than the inward momentum, indicating that feedback is not yet able to disrupt the cloud.

\subsection{Conclusions}

The changing asymmetry of the HCN lines clearly indicates a reversal of infall. Expansion motions dominate in the inner, warmer parts, and infall dominates in the outer, colder parts of the core. This can be naturally explained by the onset of feedback from massive stars. Our radiative transfer modeling demonstrates the power of constraining the source structure by fitting line shapes. A model that successfully reproduces all the features cannot have spherical symmetry, and the exact geometry probably has to be constrained by interferometric observations, to 
determine the free parameters. Although the Herschel/HIFI data do not have the desired spatial resolution, they place strong constraints on any such model, since they have to be reproduced.

We propose a scenario in which the cloud gravitationally contracts at significantly below the free-fall speed. A star cluster forms by fragmentation and accretion. The stars, especially the massive stars that dominate the luminosity, provide various feedback mechanisms to counteract the contraction. The additional pressure, be it thermal, radiative, or turbulent, decelerates the contraction, then dominates first in the inner part, before finally disrupting the whole cloud. SgrB2(M) is just beginning to drive out the gas, while large-scale global infall and probably also infall among very localized pathways in the interior remains ongoing.

This study illustrates the importance of high-frequency lines in constraining the source structure, and demonstrates the great potential of HIFI, which delivers velocity-resolved spectra at these frequencies. Ground-based telescopes may not be able to reach high enough frequencies. In our case, detecting the HCN 12-11 line was necessary in order to unambiguously trace the reversal of infall, so this result is unique to HIFI. We expect that a systematic study of high-J HCN transitions, even higher than the 12-11 line seen here, with a high signal-to-noise ratio, and toward a sample of hot core sources, would be highly rewarding in finding more sources in this particular stage of evolution.

\section{Acknowledgements}

HIFI has been designed and built by a consortium of institutes and university departments from across Europe, Canada and the United States under the leadership of SRON Netherlands Institute for Space Research, Groningen, The Netherlands and with major contributions from Germany, France and the US. Consortium members are: Canada: CSA, U.Waterloo; France: CESR, LAB, LERMA, IRAM; Germany: KOSMA, MPIfR, MPS; Ireland, NUI Maynooth; Italy: ASI, IFSI-INAF, Osservatorio Astrofisico di Arcetri- INAF; Netherlands: SRON, TUD; Poland: CAMK, CBK; Spain: Observatorio Astronmico Nacional (IGN), Centro de Astrobiologa (CSIC-INTA). Sweden: Chalmers University of Technology - MC2, RSS \& GARD; Onsala Space Observatory; Swedish National Space Board, Stockholm University - Stockholm Observatory; Switzerland: ETH Zurich, FHNW; USA: Caltech, JPL, NHSC. Support for this work was provided by NASA through an award issued by JPL/Caltech. CSO is supported by the NSF, award AST-0540882.

\subsection{Additional Information}

\subsubsection{HCN: More Frequencies}

After appearance of the paper, more bands were observed, containing additional $\mathrm{HCN}$ lines. Figures 4.6 and 4.7 show these lines as well, overlaid with the infall+expansion model. In the high-frequency bands (6 and 7), a bad baseline due to standing waves prevents a reliable comparison.

The new collisional rates of Dumouchel et al. (2010) do not make a big difference, since I was already using rates provided by Faure \& Wernli (2007, priv.comm.) instead of the old ones from Green \& Thaddeus (1974).

\subsubsection{HNC}

$\mathrm{HNC}$ as an isomer of $\mathrm{HCN}$ is routinely observed in cold molecular gas, including in galaxies. It is however an energetically unfavorable isomer: In chemical equilibrium, one would expect $10 \%$ of $\mathrm{HCN}$ to be in the form of $\mathrm{HNC}$ at $2500 \mathrm{~K}$, and a negligible fraction at much lower temperatures (Barber et al. 2002). However, interstellar molecules are not in chemical equilibrium, and the $\mathrm{HCN} / \mathrm{HNC}$ ratio is significant even at very low temperatures. In hot $(>100 \mathrm{~K})$ molecular gas the $\mathrm{HNC}$ abundance is still poorly constrained, although it is generally believed that the $\mathrm{HCN} / \mathrm{HNC}$ ratio is high in hot gas (Schilke et al. 1992). The HCN/HNC ratio is not yet understood, and poses a prominent challenge for astrochemical models.

In this context, the detection of vibrationally excited HNC (which was first detected by Schilke et al. (2003) in a proto-planetary nebula) and even $\mathrm{HN}^{13} \mathrm{C}$ in the HIFI SgrB2-M data is very interesting. Although it lies 'only' 670 $\mathrm{K}$ above the ground vibrational state (instead of $1000 \mathrm{~K}$ in the case of $\mathrm{HCN}$ ), this means that there is a significant amount of $\mathrm{HNC}$ in the warm gas.

Using the same model structure as for HCN (see Fig. 4.5), the only free parameter left is the HNC abundance. With the HNC collsional rates of Dumouchel et al. (2010), a reasonable fit could be obtained with an HCN/HNC ratio of 3 , a somewhat better fit with an $\mathrm{HNC}$ abundance of $3 \times 10^{-9}$ below $300 \mathrm{~K}$ and $7 \times 10^{-8}$ above $300 \mathrm{~K}$. This jump is especially required for the vibrationally excited $\mathrm{HN}^{13} \mathrm{C}$, whose vibrational level energies and Einstein $\mathrm{A}$ values were assumed to be the same as for HNC and whose frequencies were predicted by S. Thorwirt, based on infrared data. 


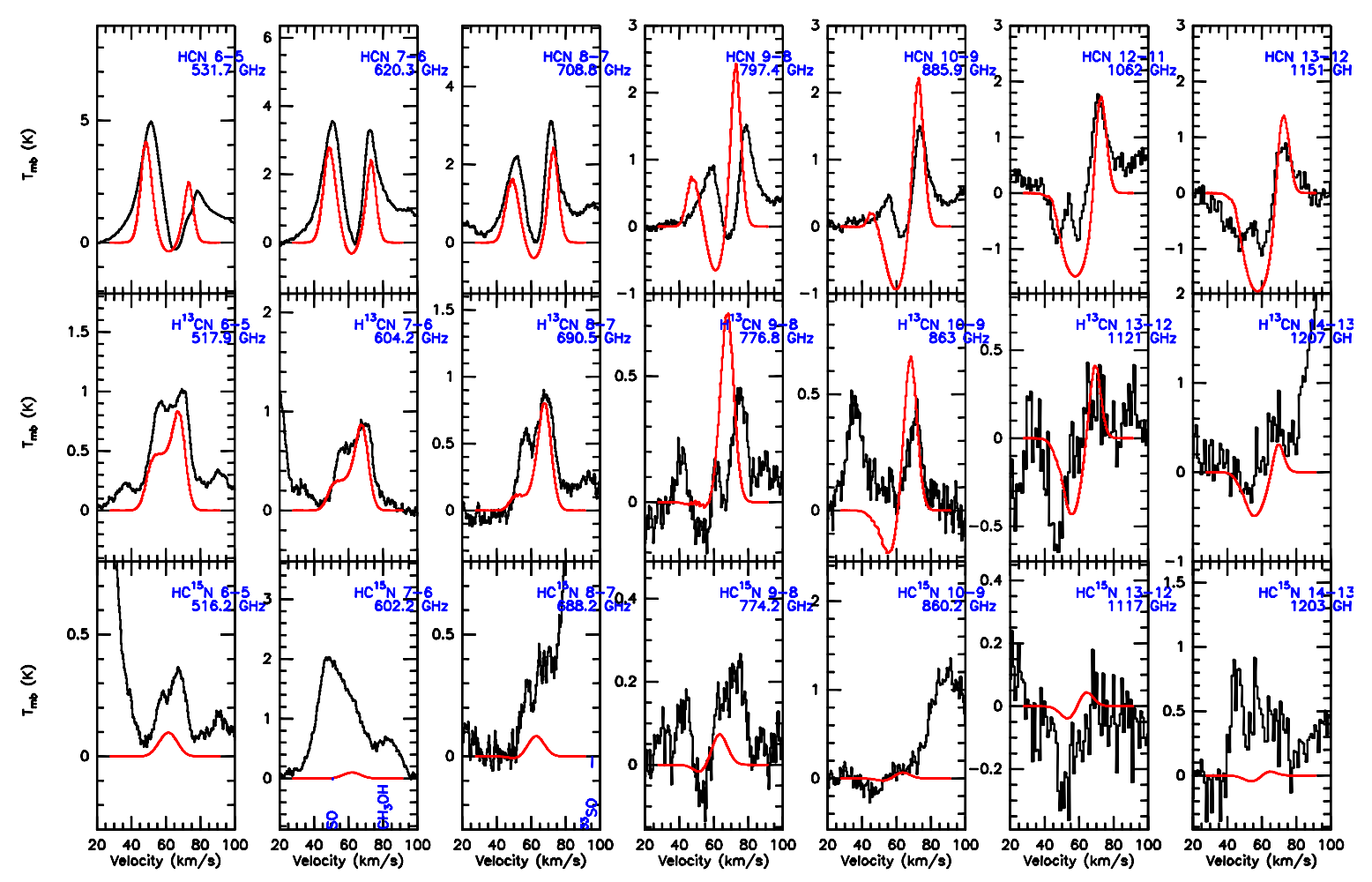

Figure 4.6: HCN lines in SgrB2-M, observed with Herschel/HIFI. The model output is overlaid in red. A source velocity of $60 \mathrm{~km} / \mathrm{s}$ was assumed.

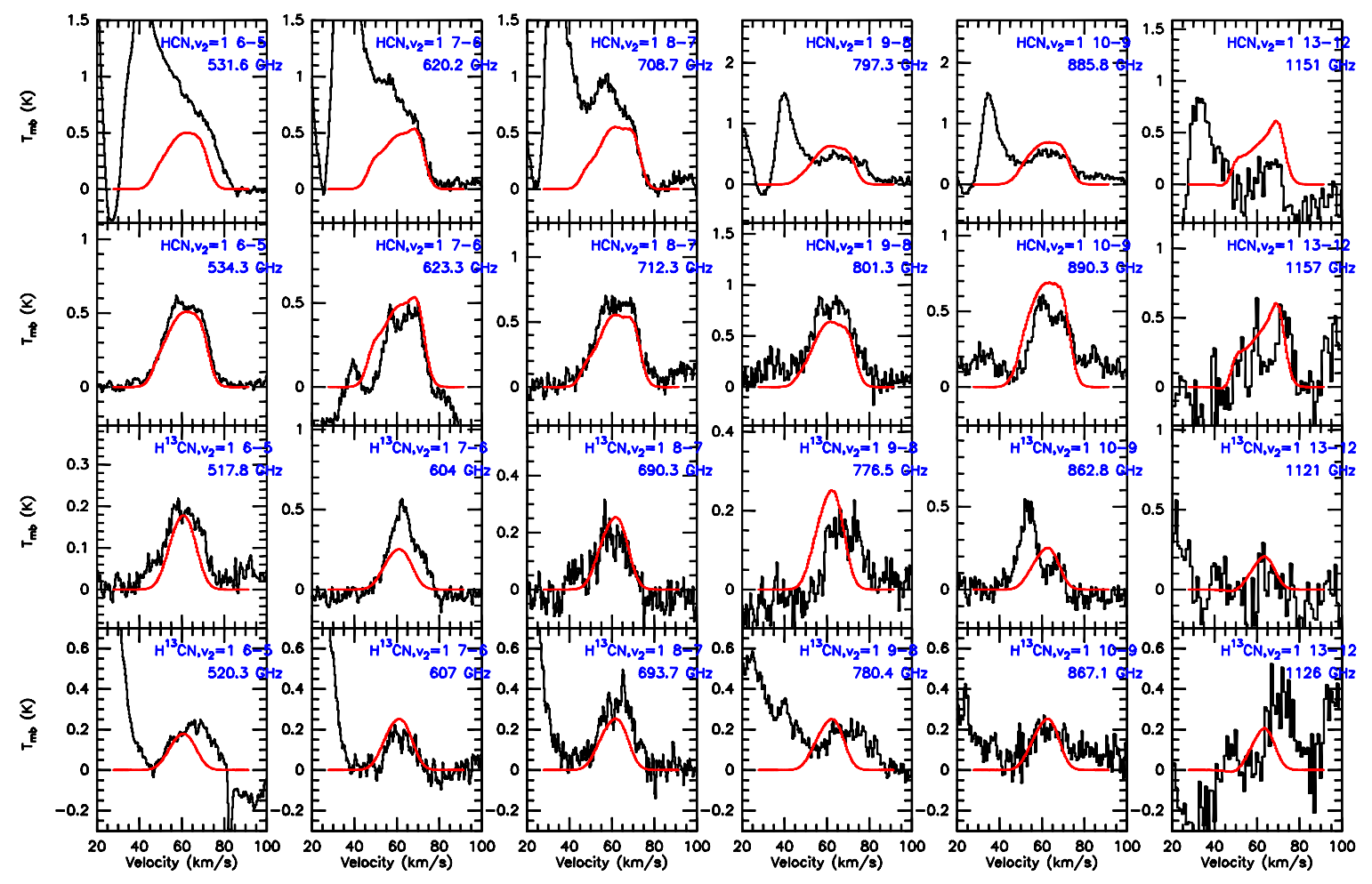

Figure 4.7: Lines from vibrationally excited $\mathrm{HCN}$ and $\mathrm{H}^{13} \mathrm{CN}$ in SgrB2-M, observed with Herschel/HIFI. The model output is overlaid in red. A source velocity of $60 \mathrm{~km} / \mathrm{s}$ was assumed. 


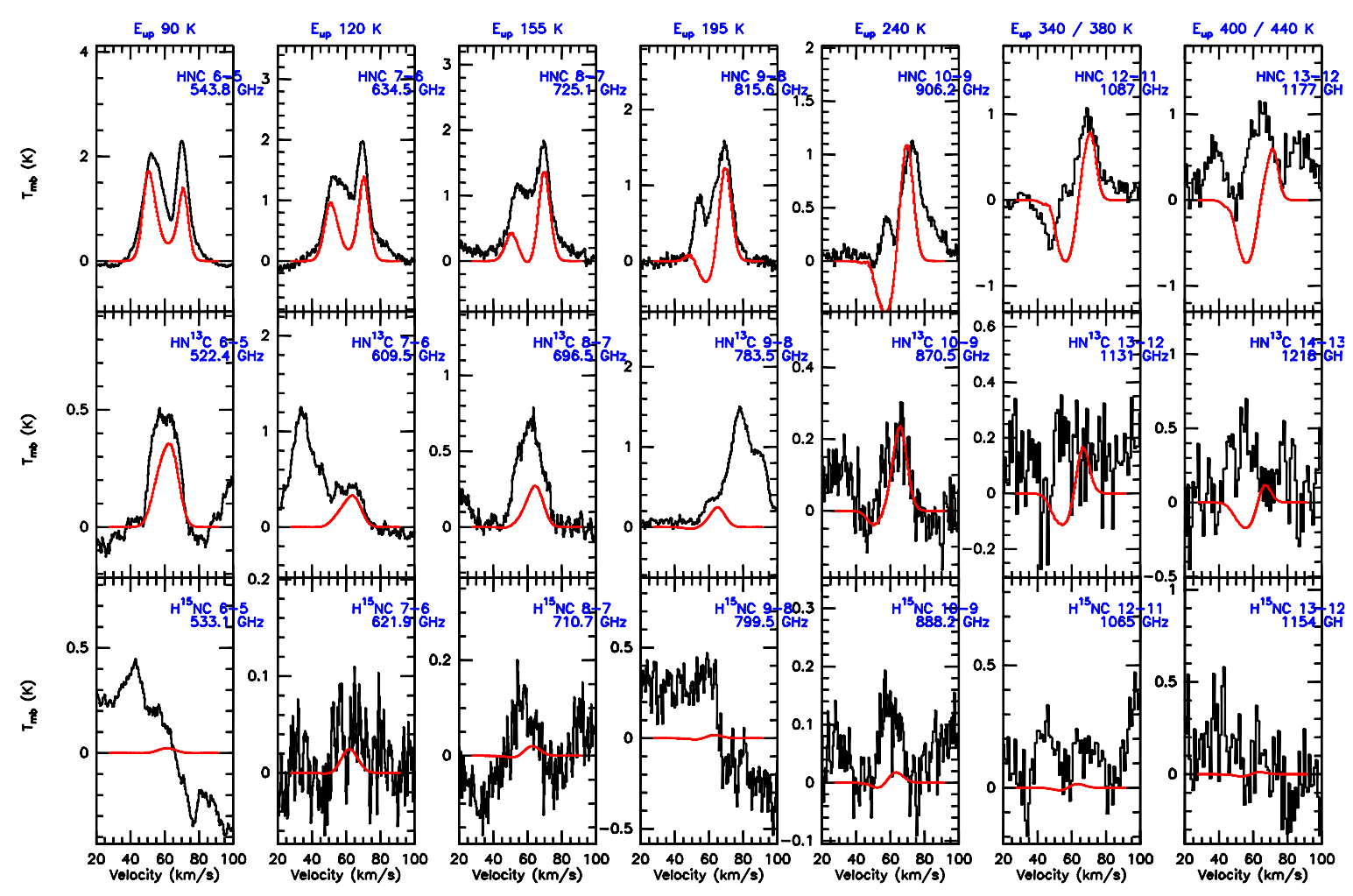

Figure 4.8: HNC lines in SgrB2-M, observed with Herschel/HIFI. The model output is overlaid in red. A source velocity of $60 \mathrm{~km} / \mathrm{s}$ was assumed.

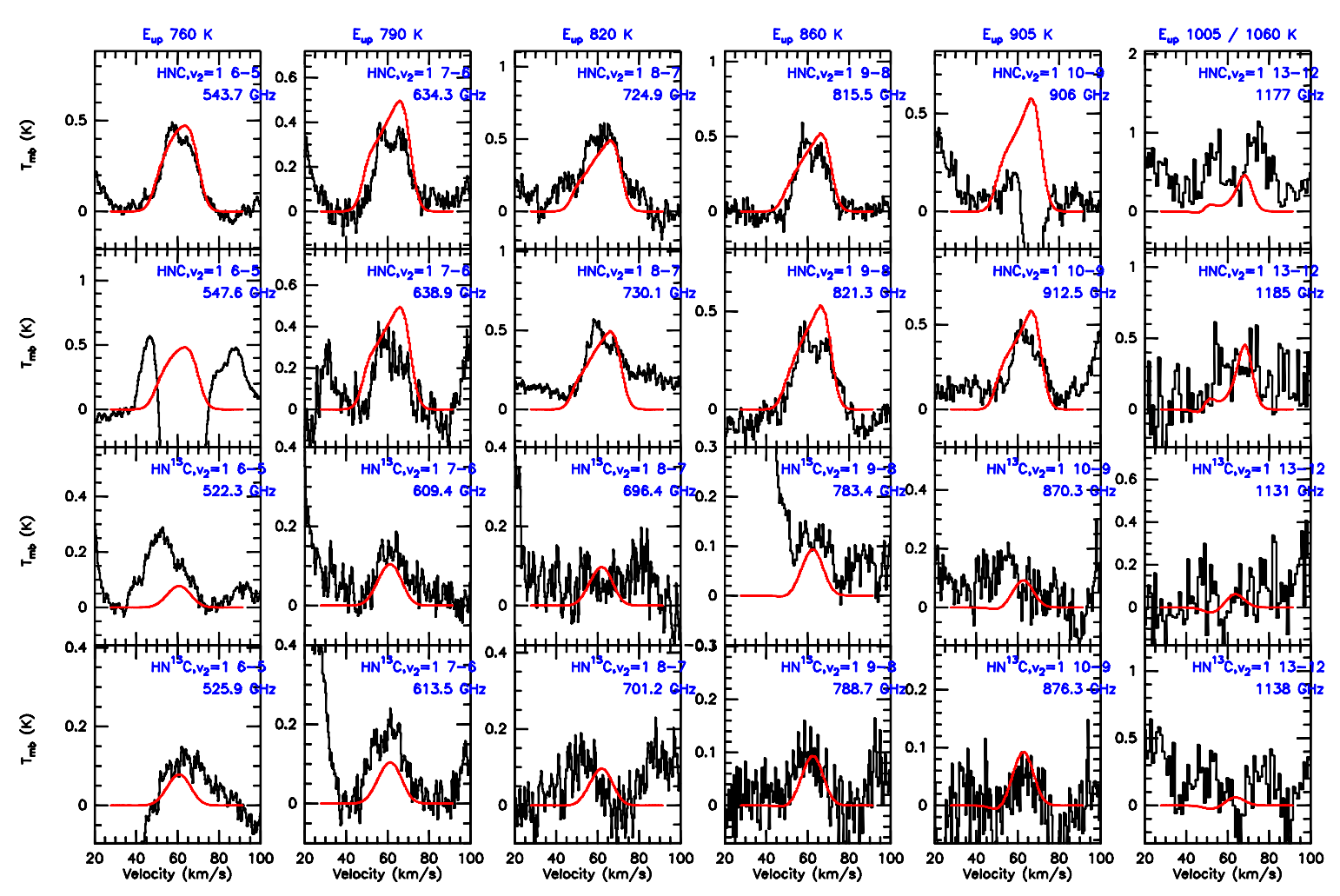

Figure 4.9: Lines from vibrationally excited $\mathrm{HNC}$ and $\mathrm{HN}^{13} \mathrm{C}$ in $\mathrm{SgrB2}-\mathrm{M}$, observed with Herschel/HIFI. The model output is overlaid in red. A source velocity of $60 \mathrm{~km} / \mathrm{s}$ was assumed. 

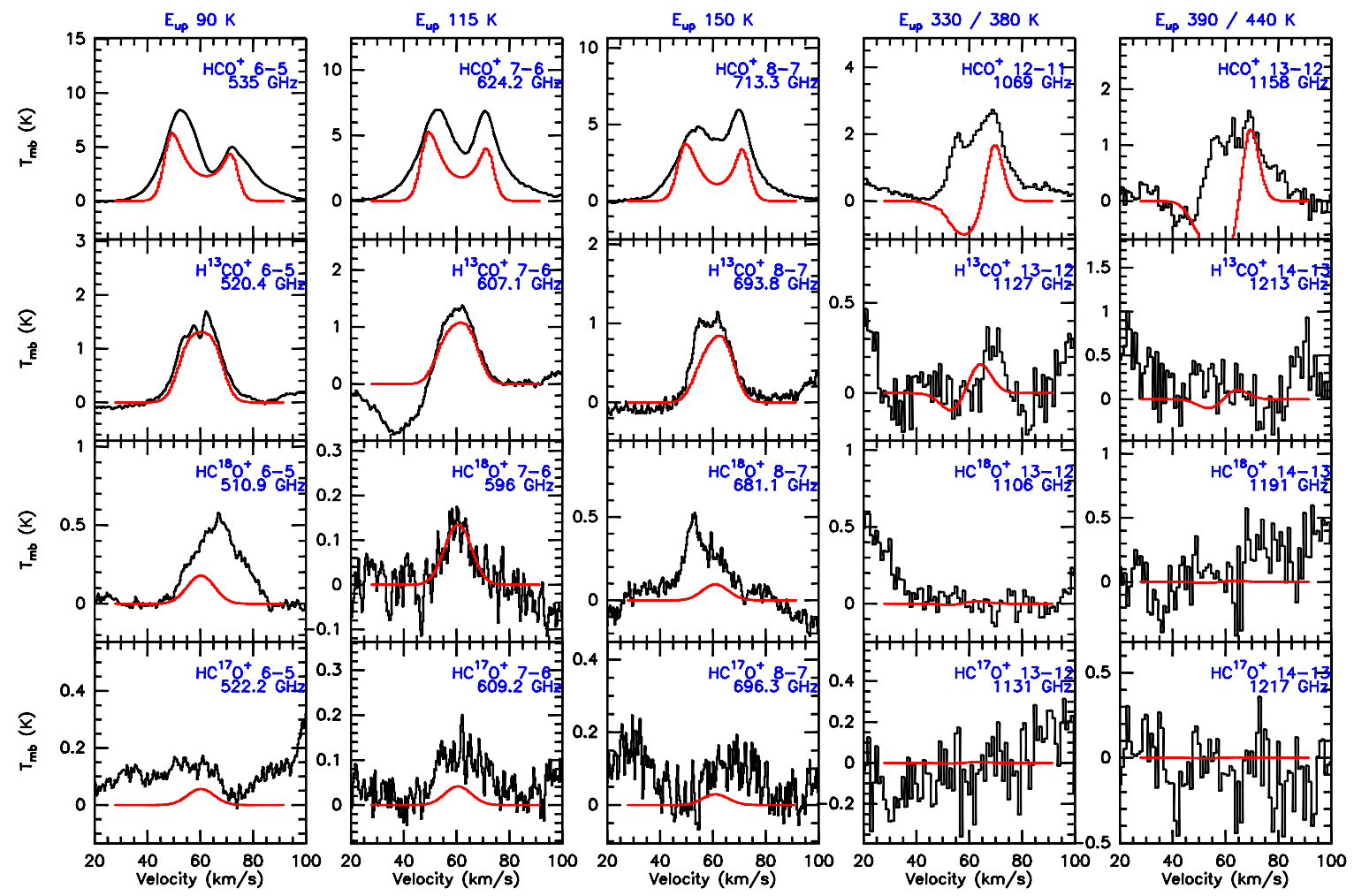

Figure 4.10: $\mathrm{HCO}^{+}$lines in SgrB2-M, observed with Herschel/HIFI. The model output is overlaid in red. A source velocity of $60 \mathrm{~km} / \mathrm{s}$ was assumed.

Figs. 4.8 and 4.9 show the spectra overlaid with the latter model. The ${ }^{14} \mathrm{~N} /{ }^{15} \mathrm{~N}$ ratio of 600 (Wilson \& Rood 1994) might be too high, as the $\mathrm{HC}^{15} \mathrm{~N}$ and $\mathrm{H}^{15} \mathrm{NC}$ spectra suggest a higher ${ }^{15} \mathrm{~N}$ abundance (or a larger extension of very high column densities).

\subsection{3 $\mathrm{HCO}^{+}$}

$\mathrm{HCO}^{+}$has a similar level structure and line strengths as $\mathrm{HCN}$, but as an ion (protonated $\mathrm{CO}$ ) it has a very different chemistry. As for HNC, it is believed that this molecule has a low abundance in hot, dense gas, but it has not been well constrained observationally. With the HIFI data set of SgrB2-M, it is possible to constrain the abundance at the high temperatures.

The $\mathrm{HCO}^{+}$vibrational state lies at around $1200 \mathrm{~K}$ above ground, $170 \mathrm{~K}$ higher in energy than $\mathrm{HCN}$. It is questionable whether the transitions are detected in these data. Figures 4.10 and 4.11 show the spectra overlaid with a model that has the same physical structure and an $\mathrm{HCO}^{+}$abundance of $10^{-9}$ below $50 \mathrm{~K}$ and $5 \times 10^{-9}$ above.

\subsubsection{Variation of the abundances}

Figure 4.12 shows the $\chi^{2}$ resulting from variations of the $\mathrm{HCN}, \mathrm{HNC}$, and $\mathrm{HCO}^{+}$abundance values. Jump temperatures of 50,100 , and $300 \mathrm{~K}$ were fixed, and the abundance in the four temperature regimes was independently varied by $\pm 20,40$, and $60 \%$.

The rather flat $\chi^{2}$ demonstrates that the abundance values are not well constrained, although in some cases the line shapes favor the central (original) value. This is also the case for HNC between 100 and $300 \mathrm{~K}$, where the $\chi^{2}$ would suggest a lower abundance (meaning less absorption in the high- $J$ transitions). The $\mathrm{HCO}^{+}$abundance above $100 \mathrm{~K}$ was, for simplicity, assumed to stay the same as below $100 \mathrm{~K}$. However, a lower abundance fits better to the lines from vibrationally excited $\mathrm{HCO}^{+}$, whose detection is questionable.

\subsubsection{Summary}

The model fits reasonably well to the $\mathrm{HCN}$ and $\mathrm{H}^{13} \mathrm{CN}$ lines from the other bands, which supports the results and conclusions drawn in the paper.

The model also fits to the $\mathrm{HNC}$ and $\mathrm{HCO}^{+}$lines when the molecular abundances are adapted. It is interesting to compare those abundances with $\mathrm{HCN}$ (Table 4.2). The HCN/HNC ratio is at least 3, with some higher value at 


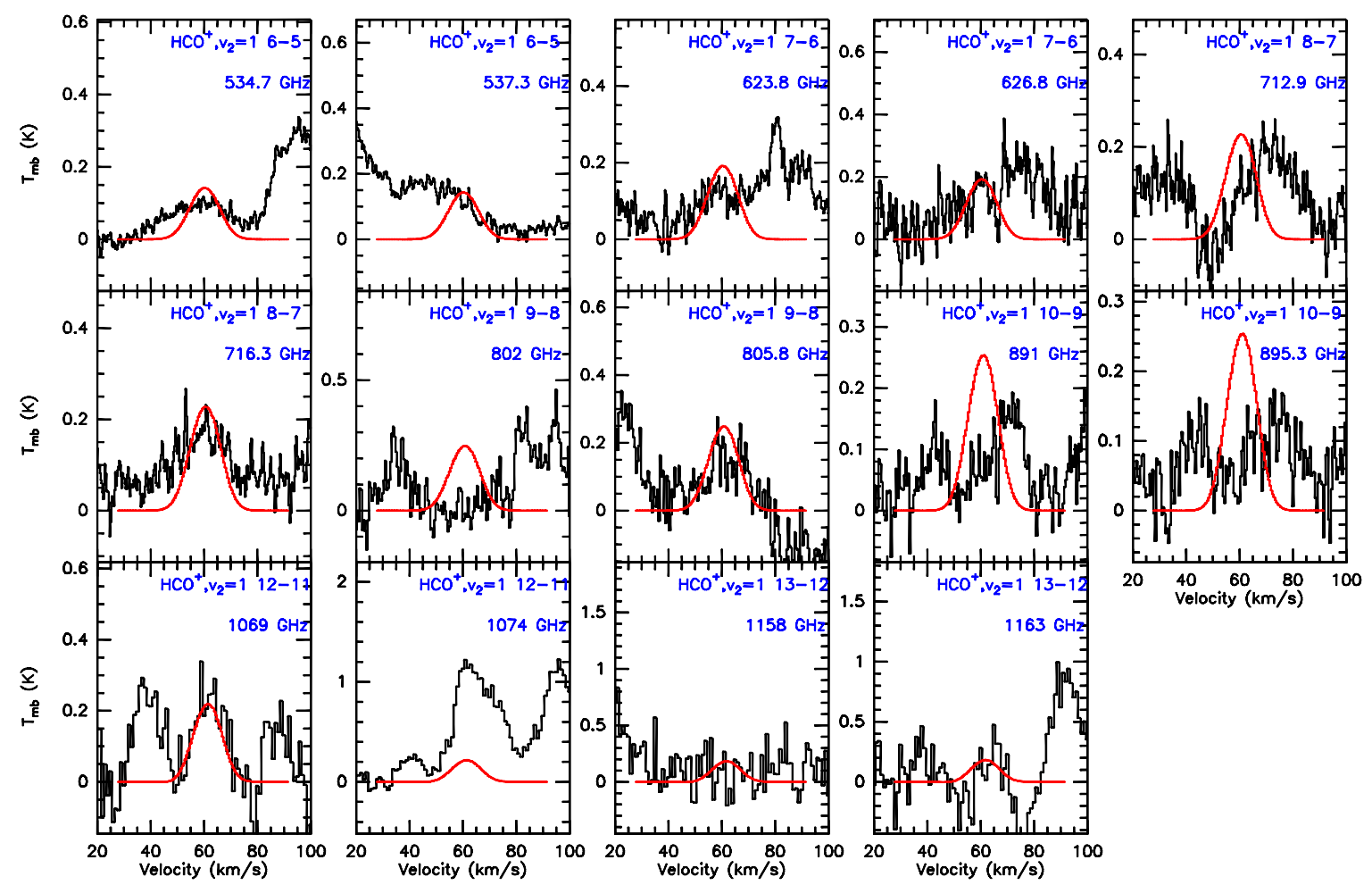

Figure 4.11: Lines from vibrationally excited $\mathrm{HCO}^{+}$in SgrB2-M, observed with Herschel/HIFI. The model output is overlaid in red. Note that the level energies and transition strengths were assumed to be the same as for HCN. A source velocity of $60 \mathrm{~km} / \mathrm{s}$ was assumed.

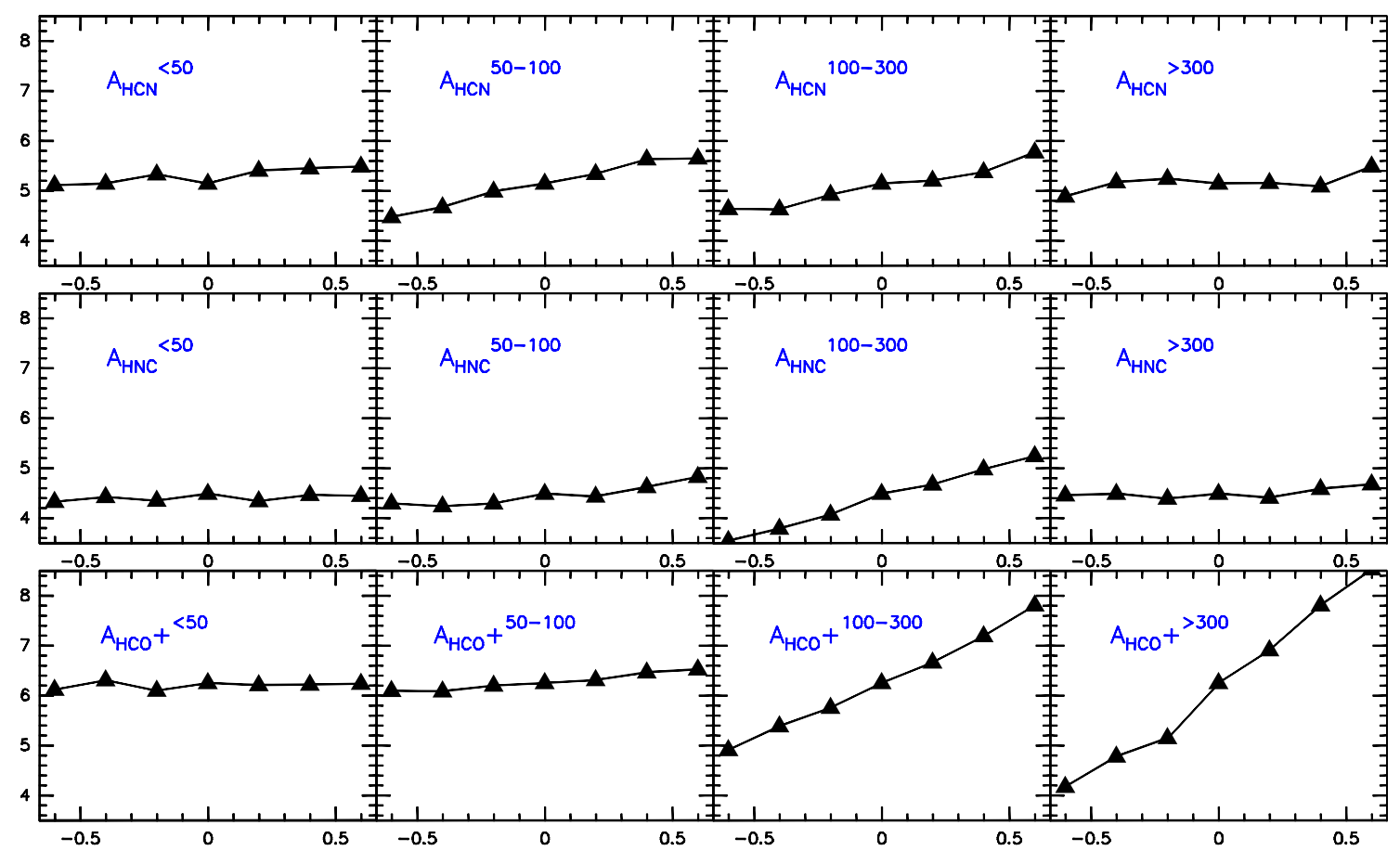

Figure 4.12: $\chi^{2}$ for the variation of the abundances in the model for SgrB2-M. 
Table 4.2: Abundances derived in SgrB2-M, relative to $\mathrm{H}_{2}$

\begin{tabular}{|l|cccc|}
\hline \hline Molecule & $<50 \mathrm{~K}$ & $50-100 \mathrm{~K}$ & $100-300 \mathrm{~K}$ & $>300 \mathrm{~K}$ \\
\hline $\mathrm{HCN}$ & $1(-8)$ & $1(-8)$ & $3(-8)$ & $3(-7)$ \\
$\mathrm{HNC}$ & $3(-9)$ & $3(-9)$ & $3(-9)$ & $7(-8)$ \\
$\mathrm{HCO}^{+}$ & $1(-9)$ & $5(-9)$ & $5(-9)$ & $5(-9)$ \\
\hline
\end{tabular}

Notes. The numbers in parentheses are powers of 10 .

the high temperatures $(>100 \mathrm{~K})$. The detection of vibrationally excited $\mathrm{HN}^{13} \mathrm{C}$ requires higher $\mathrm{HNC}$ abundances in the hot gas, as was also derived for HCN. This suggests a chemical connection between the two species.

$\mathrm{HCO}^{+}$, on the other hand, seems chemically unrelated to $\mathrm{HCN}$ and HNC. The self-absorption is not very deep, which suggests a lower abundance at low temperatures. This behavior is also predicted by Garrod et al. (2008). At high temperatures, the constant abundance adopted in the model is rather an upper limit, and would be much lower if the spectral features (Fig. 4.11) do not originate from vibrationally excited $\mathrm{HCO}^{+}$.

The abundances were derived using a manual method, i.e. changing the parameters by hand and comparing to the data by eye. This method cannot search the parameter space, nor can error estimates be given. As a hint on the errors, the $\chi^{2}$ was computed for different variations of the abundances. The exact abundance values are not well constrained, and it is entirely possible that a different combination of abundances would fit better to the data. 


\section{Chapter 5}

\section{VLA paper: Hot $\mathrm{HCN}$ around young massive stars at $0.1^{\prime \prime}$ resolution}

Sections 5.2 to 5.8 appeared in Astronomy $\mathcal{E}$ Astrophysics: Rolffs, R., Schilke, P., Wyrowski, F., Dullemond, C., Menten, K. M., Thorwirth, S., E Belloche, A. 2011, AE्FA, 529, A76+ (Rolffs et al. 2011a, received 19 January 2011 / accepted 3 March 2011)

\subsection{Overview}

Telescope: The VLA (Very Large Array) is a radio interferometer that consists of 27 antennas with $25 \mathrm{~m}$ diameter each. It is located in New Mexico and operated by NRAO (National Radio Astronomy Observatory). The interferomter cycles through four different configurations, with transitions every four months. The receiver with the shortest wavelength works at around $7 \mathrm{~mm}$. The limitations of the backend (small bandwidth and few channels) are currently overcome by new receivers in the expanded VLA project (eVLA), covering 1.2 to $50 \mathrm{GHz}$. Additional data come from the Effelsberg 100-m radio telescope in the Eifel mountains near Bonn and the IRAM (Institut de RadioAstronomie Millimétrique) 30-m millimeter-wave telescope on the Pico Veleta near Granada, Spain.

Observations: The direct $\ell$-type line of vibrationally excited HCN was observed toward three sources in 2009 during the transition from the most extended (A) to the next (B) configuration. This BnA configuration, where the northern arm is still more extended, provides a more circular beam for southern sources.

Modeling: The three-dimensional dust and line radiative transfer code RADMC-3D was used to manually adjust models to fit the data. First, the HII regions, visible in the continuum, were reproduced. Second, the density in the model was adapted in order to reproduce the line data (for fixed abundance of HCN).

Results: Both absorption against hypercompact HII regions and emission from their surroundings was detected, implying large column densities of hot $\mathrm{HCN}$ and large masses of hot gas. The models reproduce the data reasonably well, with heating only by stars in the HII regions and a Gaussian distribution of gas around them.

\subsection{Abstract}

Massive stars form deeply embedded in dense molecular gas, which they stir and heat up and ionize. During an early phase, the ionization is confined to hypercompact HII regions, and the stellar radiation is entirely absorbed by dust, giving rise to a hot molecular core. To investigate the innermost structure of such high-mass star-forming regions, we observed vibrationally excited $\mathrm{HCN}$ (via the direct $\ell$-type transition of $v_{2}=1, \Delta J=0, J=13$, which lies $1400 \mathrm{~K}$ above ground) toward the massive hot molecular cores G10.47+0.03, SgrB2-N, and SgrB2-M with the Very Large Array (VLA) at $7 \mathrm{~mm}$, reaching a resolution of about $1000 \mathrm{AU}\left(0.1^{\prime \prime}\right)$. We detect the line both in emission and in absorption against Hir regions. The latter allows to derive lower limits on the column densities of hot $\mathrm{HCN}$, which are several times $10^{19} \mathrm{~cm}^{-2}$. We see indication of expansion motions in $\mathrm{G} 10.47+0.03$ and detect velocity components in SgrB2-M at 50, 60, and $70 \mathrm{~km} \mathrm{~s}^{-1}$ relative to the Local Standard of Rest. The emission originates in regions of less than $0.1 \mathrm{pc}$ diameter around the hypercompact HII regions G10.47+0.03 B1 and SgrB2-N K2, and reaches brightness temperatures of more than $200 \mathrm{~K}$. Using the three-dimensional radiative transfer code RADMC-3D, we model the sources as dense dust cores heated by stars in the Hir regions, and derive 
Table 5.1: Observational summary

\begin{tabular}{|c|c|c|c|c|c|c|c|}
\hline \multirow[t]{2}{*}{ Source } & \multicolumn{2}{|c|}{ Pointing center } & \multirow{2}{*}{$\begin{array}{c}\text { Continuum beam }{ }^{a} \\
\left({ }^{\prime \prime} ;{ }^{\circ}\right)\end{array}$} & \multirow{2}{*}{$\begin{array}{l}\text { Line beam }{ }^{a} \\
\left({ }^{\prime \prime} ;{ }^{\circ}\right)\end{array}$} & \multirow{2}{*}{$\begin{array}{c}\mathrm{rms} 1 \mathrm{~km} \mathrm{~s}^{-1 b} \\
(\mathrm{mJy} / \text { Beam })\end{array}$} & \multirow{2}{*}{$\begin{array}{l}\text { rms cont. }^{b} \\
(\mathrm{~K})\end{array}$} & \multirow{2}{*}{$\begin{array}{r}\text { rms int. line }^{b} \\
\left(\mathrm{~K} \mathrm{~km} \mathrm{~s}^{-1}\right)\end{array}$} \\
\hline & $\mathrm{RA}(\mathrm{J} 2000)$ & $\operatorname{Dec}(\mathrm{J} 2000)$ & & & & & \\
\hline G10.47+0.03 & 180838.236 & -195150.3 & $0.12 \times 0.06 ; 82$ & $0.14 \times 0.1 ; 69$ & 2.3 & 45 & 900 \\
\hline SgrB2-N & 174719.902 & -282217.8 & $0.12 \times 0.08 ; 60$ & $0.15 \times 0.11 ; 51$ & 3.0 & 75 & 1300 \\
\hline SgrB2-M & 174720.202 & -282305.3 & $0.12 \times 0.08 ; 63$ & $0.15 \times 0.11 ; 51$ & 3.3 & 95 & 1400 \\
\hline
\end{tabular}

Notes. ${ }^{(a)}$ Different weighting schemes were used for continuum and line images. Major and minor axis and position angle are given. ${ }^{(b)} \mathrm{rms}$ noise in $1 \mathrm{~km} \mathrm{~s}^{-1}$ channel, continuum and integrated line maps (Figs. 5.2, 5.4, and 5.6). The noise is evaluated over a box from -2 to -1 " in both RA and Dec relative to the phase center.

masses of hot $(>300 \mathrm{~K})$ molecular gas of more than 100 solar masses (for an $\mathrm{HCN}$ fractional abundance of $10^{-5}$ ), challenging current simulations of massive star formation. Heating only by the stars in the HII regions is sufficient to produce such large quantities of hot molecular gas, provided that dust is optically thick to its own radiation, leading to high temperatures through diffusion of radiation.

\subsection{Introduction}

The formation of massive stars occurs deep inside dense molecular cloud cores. The high luminosity of massive (proto)stars stirs and heats up the surrounding gas, leading to the evaporation of ice mantles around dust grains and to high degrees of excitation of the molecules. This stage, just prior to ionization and destruction of the molecular gas, is characterized by strong line and dust emission from a compact region, and is called a hot molecular core (e.g. Cesaroni 2005). Since these sources are short-lived, they are rare and hence far away, which hampers the determination of their physical structure (density, temperature, velocity, molecular abundances). Nonetheless, such knowledge is a key information for understanding the process of massive star formation (Zinnecker \& Yorke 2007) and for the interpretation of molecular line surveys (e.g. Schilke et al. 2006; Belloche et al. 2007; Bergin et al. 2010), and has been constrained by radiative transfer modeling of both single-dish (e.g. Hatchell \& van der Tak 2003; Rolffs et al. 2011b) and interferometry observations (e.g. Osorio et al. 2009).

Especially interesting is the hottest molecular gas, which is closest to the heating sources. A good tracer is vibrationally excited $\mathrm{HCN}\left(v_{2}=1\right)$, whose rotationally excited levels lie $\sim 1000 \mathrm{~K}$ higher in energy than the ground vibrational state, and which has transitions in the $\mathrm{cm}$-wave regime, whose radiation is not obscured by dust. These so-called direct $\ell$-type transitions are intrinsically weak, but have been detected in Galactic high-mass star-forming regions (Thorwirth 2001) as well as toward the proto-planetary nebula CRL618 (Thorwirth et al. 2003b) and the starburst galaxy Arp220 (Salter et al. 2008).

Obviously, this hot gas is very compact, and high spatial resolution is needed to investigate it. In this paper, we present VLA observations (with among the highest resolution ever obtained for thermal molecular line emission) of three massive hot cores which have strong HCN direct $\ell$-type lines (as measured with the Effelsberg 100-m telescope, Sect. 5.5.4). The sources are G10.47+0.03 (e.g. Wyrowski et al. 1999), which lies at a distance of 10.6 kpc (Pandian et al. 2008) and has a luminosity of about $7 \times 10^{5} \mathrm{~L}_{\odot}$ (Cesaroni et al. 2010), and SgrB2-N and -M near the Galactic center at $7.8 \mathrm{kpc}$ distance (Reid et al. 2009), which have total luminosities of about $8.4 \times 10^{5}$ and $6.3 \times 10^{6} \mathrm{~L}_{\odot}$, respectively (Goldsmith et al. 1992). All sources are sites of very active high-mass star formation and contain several HII regions.

After describing observations and data reduction (Sect. 5.4), we present the results (Sect. 5.5) and the radiative transfer modeling (Sect. 5.6), discuss the implications (Sect. 5.7) and draw conclusions (Sect. 5.8).

\subsection{Observations and data reduction}

\subsubsection{VLA}

With the National Radio Astronomy Observatory's (NRAO's) ${ }^{1}$ Very Large Array (VLA) in its BnA configuration we observed three massive star forming regions in the direct $\ell$-type line of vibrationally excited $\mathrm{HCN}$ at 40.7669 $\mathrm{GHz}$ (project AR687). This transition connects the two levels of the $v_{2}=1, J=13$ doublet, whose energies lie 1411 and $1413 \mathrm{~K}$ above the ground state, respectively.

Each of the sources was observed during LST 15-21; G10.47+0.03 on 2009 January 30, SgrB2-M on January 31, and SgrB2-N on February 1. Bandpass calibration was done on 3C279 and 3C454.3, and primary flux cali-

\footnotetext{
${ }^{1}$ The National Radio Astronomy Observatory is a facility of the National Science Foundation operated under cooperative agreement by Associated Universities, Inc.
} 
bration on $3 \mathrm{C} 286$. The observations were performed in fast-switching mode with $150 \mathrm{~s}$ on the source and 30s on a phase calibrator (1755-225 for G10.47+0.03 and SgrA* for SgrB2), yielding an on-source integration time of about 4.5 hours per source. For amplitude calibration, 1733-130 (G10.47+0.03) or SgrA* (SgrB2) was observed every 20 minutes, and reference pointing was done every hour on these sources.

For G10.47+0.03, we centered one IF with $6.25 \mathrm{MHz}$ bandwidth at $40.762 \mathrm{GHz}$ (shifted from the rest frequency due to the apparent source motion) and one with $25 \mathrm{MHz}$ bandwidth at $40.78 \mathrm{GHz}$ to increase the continuum sensitivity. For the SgrB2 sources, the line is broader, so we used two overlapping IFs with $6.25 \mathrm{MHz}$ bandwidth centered at $40.761 \mathrm{GHz}$ and $40.765 \mathrm{GHz}$. The channel width is $0.72 \mathrm{~km} \mathrm{~s}^{-1}$.

Calibration and editing was done in AIPS: Bad data points were flagged; phase, amplitude, and flux were calibrated using the continuum data of the calibrator sources, and bandpass calibration was applied. In MIRIAD 2 (Sault et al. 1995), Doppler correction was done and the channels were averaged to a width of $1 \mathrm{~km} \mathrm{~s}^{-1}$, covering LSR velocities of $48-87 \mathrm{~km} \mathrm{~s}^{-1}$ in G10.47+0.03 and $27-93 \mathrm{~km} \mathrm{~s}^{-1}$ in SgrB2. The continuum was fitted using linefree channels (excluding 58-77, 44-80, and 42-82 $\mathrm{km} \mathrm{s}^{-1}$ in G10.47+0.03, SgrB2-N, and SgrB2-M, respectively) and subtracted from the line data. Imaging was done with almost uniform weighting for continuum maps (MIRIAD robust parameter -2 , resulting in beams of $0.12^{\prime \prime} \times 0.06^{\prime \prime}$ for G10.47 and $0.12^{\prime \prime} \times 0.08^{\prime \prime}$ for SgrB2) and more natural weighting for line maps (robust 0.5 , resulting in beams of $0.14^{\prime \prime} \times 0.1^{\prime \prime}$ for G10.47 and $0.15^{\prime \prime} \times 0.11^{\prime \prime}$ for SgrB2). For total fluxes and lower noise levels, the continuum was also imaged in natural weighting.

A pixel size of $0.02^{\prime \prime}$ and a cutoff for cleaning of twice the rms noise in the image was used. Table 5.1 summarizes beam sizes and noise levels.

For G10.47+0.03, the continuum clean components were used to self-calibrate the phases and apply the solutions to the line data. The absorption lines in this source were almost invisible before self-calibration. The procedure did not improve the SgrB2 data, presumably due to the good calibration with SgrA* only $0.7^{\circ}$ away. All figures shown in this paper were created with the GILDAS software ${ }^{3}$.

\subsubsection{Effelsberg}

With the Effelsberg 100-m telescope of the Max-Planck-Institut für Radioastronomie, we observed several sources in HCN direct $\ell$-type lines in 2000 (Thorwirth 2001), 2002, and 2007. The lines were the $J=9$ transition at 20.18 $\mathrm{GHz}, J=10$ at $24.66 \mathrm{GHz}, J=11$ at $29.58 \mathrm{GHz}$, and $J=12$ at $34.95 \mathrm{GHz}$. Beam sizes are 37, 31, 26, and 22", respectively. Pointing was done on nearby quasars, and is accurate to about $10^{\prime \prime}$. The different polarizations and scans were averaged, and a baseline was subtracted by fitting a polynomial of order 0 to 5 to line-free channels. The noise tube units measured by the telescope were converted to fluxes by comparison with a source of known flux, mostly NGC7027 (Ott et al. 1994), which was observed once per day. To correct the deformation of the telescope at low elevations, the resulting flux was multiplied by an additional factor of $\left(1-0.01 \times\left(30^{\circ}-e l v\right)\right)^{-1}$ (Gallimore et al. 2001), which is 1.25 at an elevation of $10^{\circ}$, typical for SgrB2.

The observations were complemented by data from the millimeter-wave line survey of SgrB2 with the IRAM 30-m telescope (Belloche et al. 2008).

\subsection{Results}

For each source we show spectra (Fig. 5.1), continuum and integrated line maps, as well as position-velocity diagrams. Absorption lines are summarized in Table 5.2 and emission lines in Table 5.3.

From Gaussian fits to the absorption lines, lower limits on the optical depth $\tau$ and on the column density of hot $\mathrm{HCN}$ can be derived: The line-to-continuum ratio is $e^{-\tau}$ if line emission can be neglected, i.e. if the beam-averaged (excitation) temperature of the absorbing gas is much lower than the beam-averaged background brightness temperature - else the line-to-continuum ratio is larger than $e^{-\tau}$. The background radiation is provided by free-free emission from ionized gas of around $10^{4} \mathrm{~K}$, which is at least an order of magnitude larger than the temperature of the absorbing gas. However, the observed continuum is lower than $10^{4} \mathrm{~K}$ due to optically thin freefree radiation in parts of the beam (see e.g. Cesaroni et al. (2010) for spectral energy distributions of HII regions with density gradient). Additionally, the hot molecular gas could emit over the whole beam. Therefore, the real optical depths can be much higher than derived from the line-to-continuum ratio, and the values given in Table 5.2 are only lower limits.

From $\tau$ and the observed line width $\Delta v$ (FWHM), the HCN column density can be derived, assuming Local Thermodynamic Equilibrium (LTE, see also Sect. 5.6.1) at a temperature $T$ : Using

$$
\tau=\frac{c^{2}}{8 \pi v^{2}} N_{\mathrm{u}} A_{\mathrm{ul}} \Phi\left(e^{\frac{h v}{k T}}-1\right)
$$

\footnotetext{
${ }^{2}$ http://bima.astro.umd.edu/miriad

${ }^{3}$ http://www.iram.fr/IRAMFR/GILDAS
} 

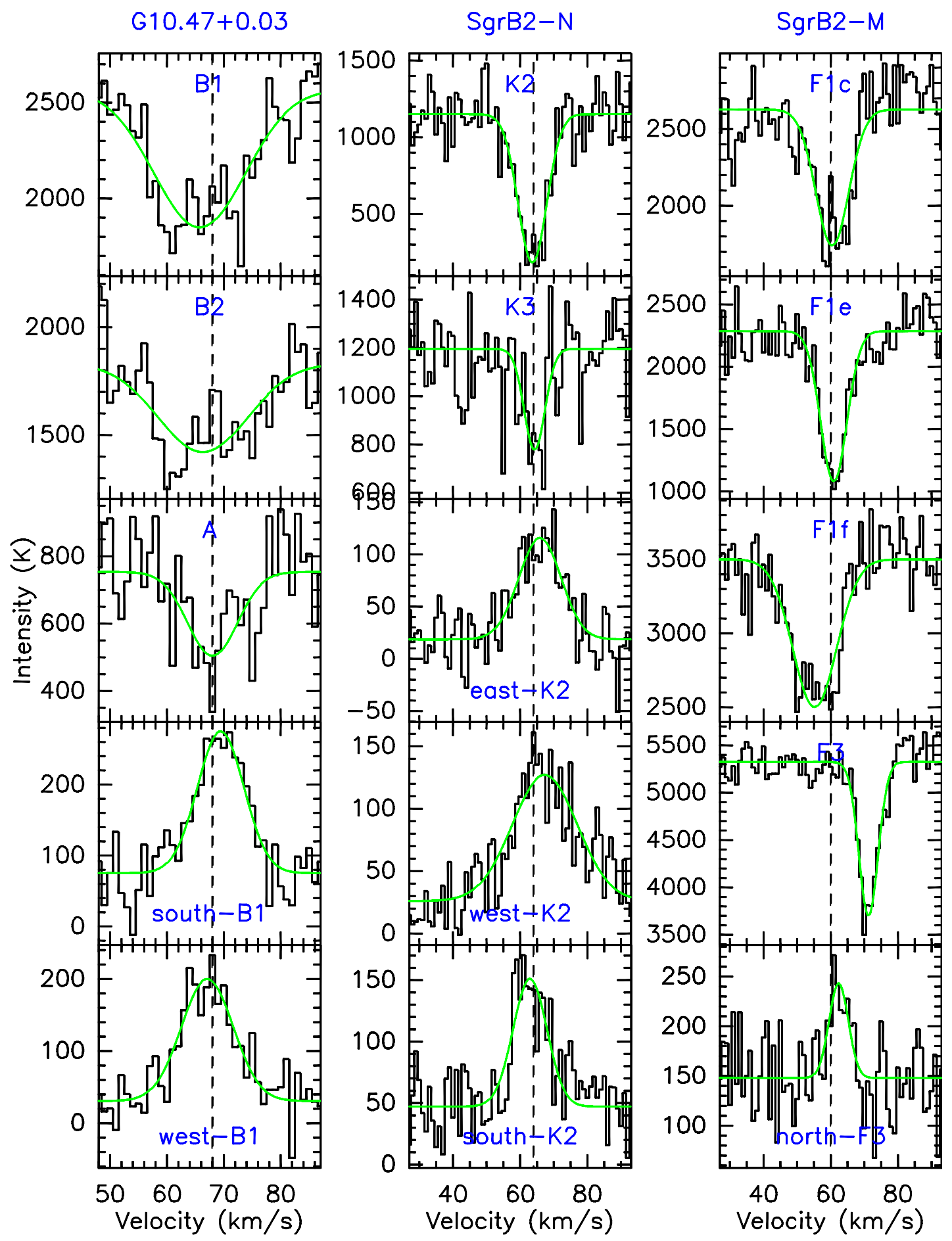

Figure 5.1: The $v_{2}=1, J=13$ direct $\ell$-type line of $\mathrm{HCN}$ observed toward several positions in G10.47+0.03 (left column), SgrB2-N (central column), and SgrB2-M (right column). The emission lines are averaged over the areas shown in Figs. 5.2, 5.4, and 5.6, while the absorption lines are observed with the beams given in Table 5.1. Overlaid in green are Gaussian fits, whose parameters are given in Tables 5.2 and 5.3. The vertical dashed lines mark the assumed systemic velocities. 


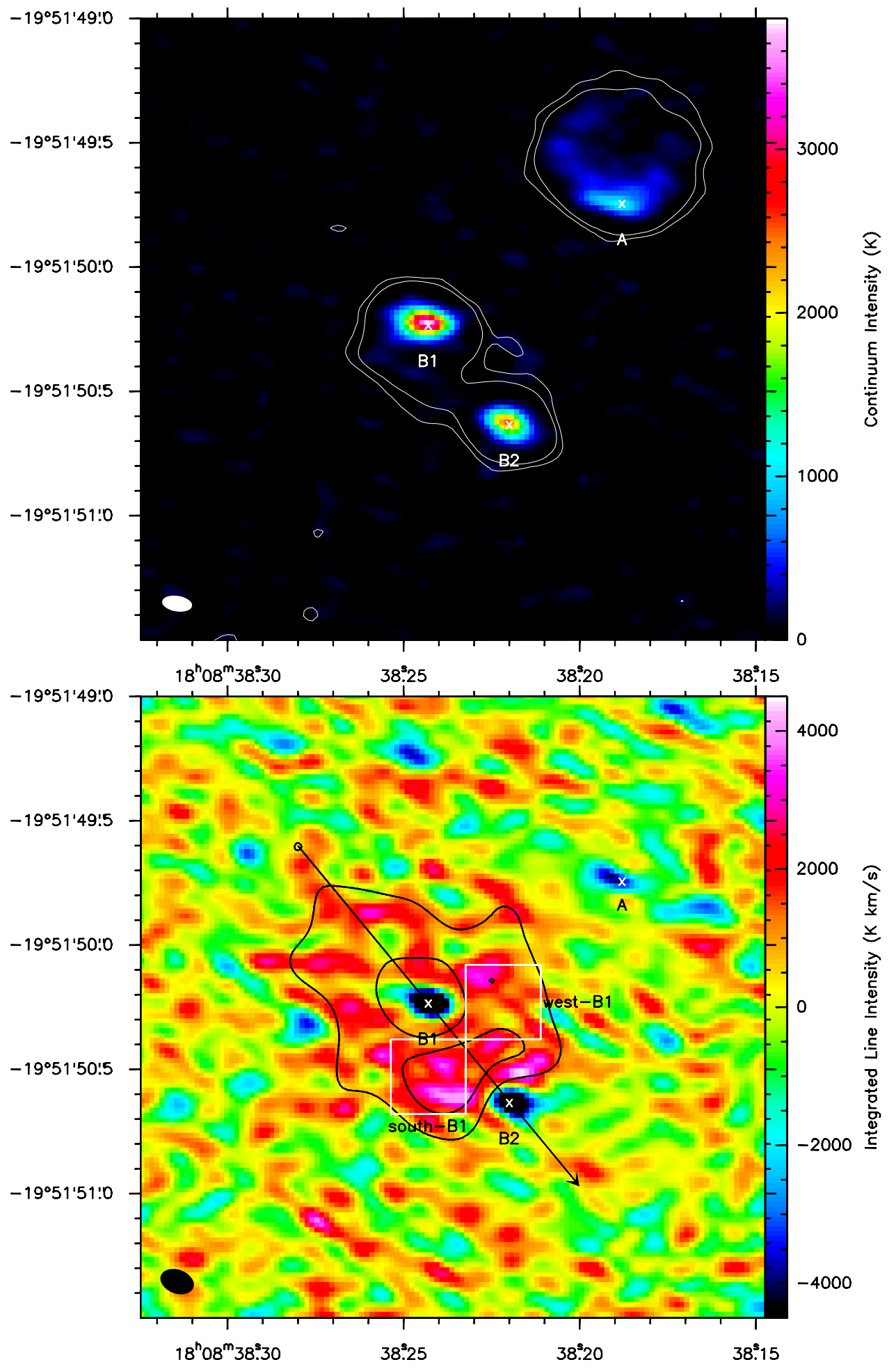

Figure 5.2: VLA maps of G10.47+0.03. The $7 \mathrm{~mm}$ continuum emission is shown on the top, the integrated line map on the bottom (beams are depicted in the lower left). Contours denote 3 and $6 \sigma$ levels of the naturally weighted continuum (white, top) and of the integrated line emission convolved to $0.25^{\prime \prime}$ resolution (black, bottom). The white crosses give the positions toward which the absorption spectra were obtained and the white boxes the area over which the emission spectra are integrated, shown in Fig. 5.1. The arrow denotes the direction along which the position-velocity plot of Fig. 5.3 was obtained. The map size is $2.5^{\prime \prime}$, about 0.13 pc. 
Table 5.2: Parameters of absorption lines shown in Fig. 5.1

\begin{tabular}{lcccccccc}
\hline \hline Hit region & $\begin{array}{c}\text { RA } \\
(\mathrm{J} 2000)\end{array}$ & $\begin{array}{c}\text { Dec. } \\
(\mathrm{J} 2000)\end{array}$ & $\begin{array}{c}\text { cont. }^{a} \\
(\mathrm{~K})\end{array}$ & $\begin{array}{c}\text { line width }^{b} \\
\left(\mathrm{~km} \mathrm{~s}^{-1}\right)\end{array}$ & $\begin{array}{c}\text { velocity } \\
\left(\mathrm{km} \mathrm{s}^{-1}\right)\end{array}$ & $\begin{array}{c}\text { line/cont. } \\
\tau\end{array}$ & $\begin{array}{c}\text { HCN column density }^{c} \\
\left(\mathrm{~cm}^{-2}\right)\end{array}$ \\
\hline G10.47+0.03 B1 & 180838.243 & -195150.22 & 2600 & 18.9 & 65.8 & 0.72 & 0.33 & $5.5(19)$ \\
G10.47+0.03 B2 & 180838.22 & -195150.62 & 1800 & 18.5 & 66.4 & 0.776 & 0.25 & $4.1(19)$ \\
G10.47+0.03 A & 180838.188 & -195149.74 & 750 & 10.3 & 68 & 0.67 & 0.4 & $3.6(19)$ \\
\hline SgrB2-N K2 & 174719.882 & -282218.45 & 1150 & 10.0 & 63.6 & 0.16 & 1.83 & $1.6(20)$ \\
SgrB2-N K3 & 174719.902 & -282217.18 & 1200 & 7.3 & 64.3 & 0.652 & 0.43 & $2.8(19)$ \\
\hline SgrB2-M F1c & 174720.126 & -282303.9 & 2650 & 11.5 & 60.4 & 0.664 & 0.41 & $4.1(19)$ \\
SgrB2-M F1e & 174720.131 & -282304.02 & 2300 & 9.2 & 60.9 & 0.474 & 0.75 & $6.1(19)$ \\
SgrB2-M F1f & 174720.147 & -282303.88 & 3500 & 15.3 & 55.2 & 0.714 & 0.34 & $4.4(19)$ \\
SgrB2-M F3 & 174720.172 & -282304.58 & 5350 & 6.7 & 71.2 & 0.696 & 0.36 & $2.1(19)$ \\
\hline
\end{tabular}

Notes. The beam size is $0.14^{\prime \prime} \times 0.1^{\prime \prime}$ for G10.47+0.03 and $0.15^{\prime \prime} \times 0.11^{\prime \prime}$ for SgrB2. The rms on $1 \mathrm{~km} \mathrm{~s}^{-1}$ channels is $120-150$ K. Results of Gaussian fits and lower limits on the optical depth $\tau$ and on the column density of hot HCN are given. ${ }^{(a)}$ The continuum flux density is the brightness temperature at the given locations in the line maps (which have a larger beam than the pure continuum maps). To convert from K to mJy/Beam, one has to divide by 53 for G10.47+0.03 and by 45 for SgrB2. ${ }^{(b)}$ Full Width at Half Maximum (FWHM) of the Gaussian ${ }^{(c)}$ The numbers in parentheses are powers of 10.

Table 5.3: Results of Gaussian fits to the emission lines shown in Fig. 5.1

\begin{tabular}{lccc}
\hline \hline field & $\begin{array}{c}\text { line width } \\
\left(\mathrm{km} \mathrm{s}^{-1}\right)\end{array}$ & $\begin{array}{c}\text { velocity } \\
\left(\mathrm{km} \mathrm{s}^{-1}\right)\end{array}$ & $\begin{array}{c}\text { intensity } \\
(\mathrm{K})\end{array}$ \\
\hline G10.47+0.03 south-B1 & 9.7 & 69.4 & 200 \\
G10.47+0.03 west-B1 & 10.8 & 67.1 & 170 \\
\hline SgrB2-N east-K2 & 14.9 & 65.9 & 100 \\
SgrB2-N west-K2 & 22.9 & 67.3 & 100 \\
SgrB2-N south-K2 & 11.9 & 62.9 & 100 \\
\hline SgrB2-M north-F3 & 6.8 & 62.4 & 100 \\
\hline
\end{tabular}

Notes. ${ }^{(a)}$ Full Width at Half Maximum (FWHM) of the Gaussian

with frequency $v=40.7669 \mathrm{GHz}$, Einstein A coefficient $A_{\mathrm{ul}}=3.75 \times 10^{-8} \mathrm{~s}^{-1}$ and profile function at Gaussian line center $\Phi=\sqrt{\frac{\ln (2)}{\pi}} \times \frac{2}{\Delta v} \approx \frac{0.94}{\Delta v}$, the total column density $N$ can be obtained from the column density in the upper state

$$
N_{\mathrm{u}}=N \frac{g_{\mathrm{u}}}{Q(T)} e^{-\frac{E_{u}}{k T}}
$$

inserting the statistical weight $g_{\mathrm{u}}=2 J+1=27$, the energy of the upper level $E_{\mathrm{u}}=1413 \mathrm{~K}$, and the partition function $Q(T)$ (Barber et al. 2002). The temperature has two competing effects on these equations: While a higher temperature raises $N_{\mathrm{u}}$, it lowers the population difference between upper and lower level (last factor of Equation 5.1). For given $\tau$ and $\Delta v$, a minimum of $N$ is reached around $500 \mathrm{~K}$, while twice as many molecules are required at around 300 and $1000 \mathrm{~K}$, and 10 times as many at 200 and $1800 \mathrm{~K}$ (100 times at $140 \mathrm{~K}$ ). In Table 5.2, we give this minimum, which can be regarded as a lower limit on the column density of hot HCN. Only if the level population difference is higher than expected from LTE, the true HCN column density would be lower.

\subsubsection{G10.47+0.03}

The 7mm continuum (Fig. 5.2 top) shows the two hypercompact Hir regions B1 and B2 as well as the cometaryshaped ultracompact Hir region A (see also Cesaroni et al. 2010). The total fluxes are $80 \mathrm{mJy}$ for B1, $48 \mathrm{mJy}$ for B2, and $79 \mathrm{mJy}$ for A.

The integrated line map (Fig. 5.2 bottom) shows the absorption and the distribution of line emission, which is concentrated around B1 and especially strong between B1 and B2. The absorption lines toward B1 and B2 have a component at lower velocities than the systemic velocity of around $68 \mathrm{~km} \mathrm{~s}^{-1}$ (derived from emission lines), indicating expansion motions (Figs. 5.1 and 5.3).

\subsubsection{SgrB2-N}

The continuum map (Fig. 5.4 top) shows K2 and K3 (Gaume et al. 1995) with fluxes of 70 and $140 \mathrm{mJy}$, respectively. Outside of the range shown in Fig. 5.4, we also detect K4, K1 (as a north-south elongated, 0.5" long HII 


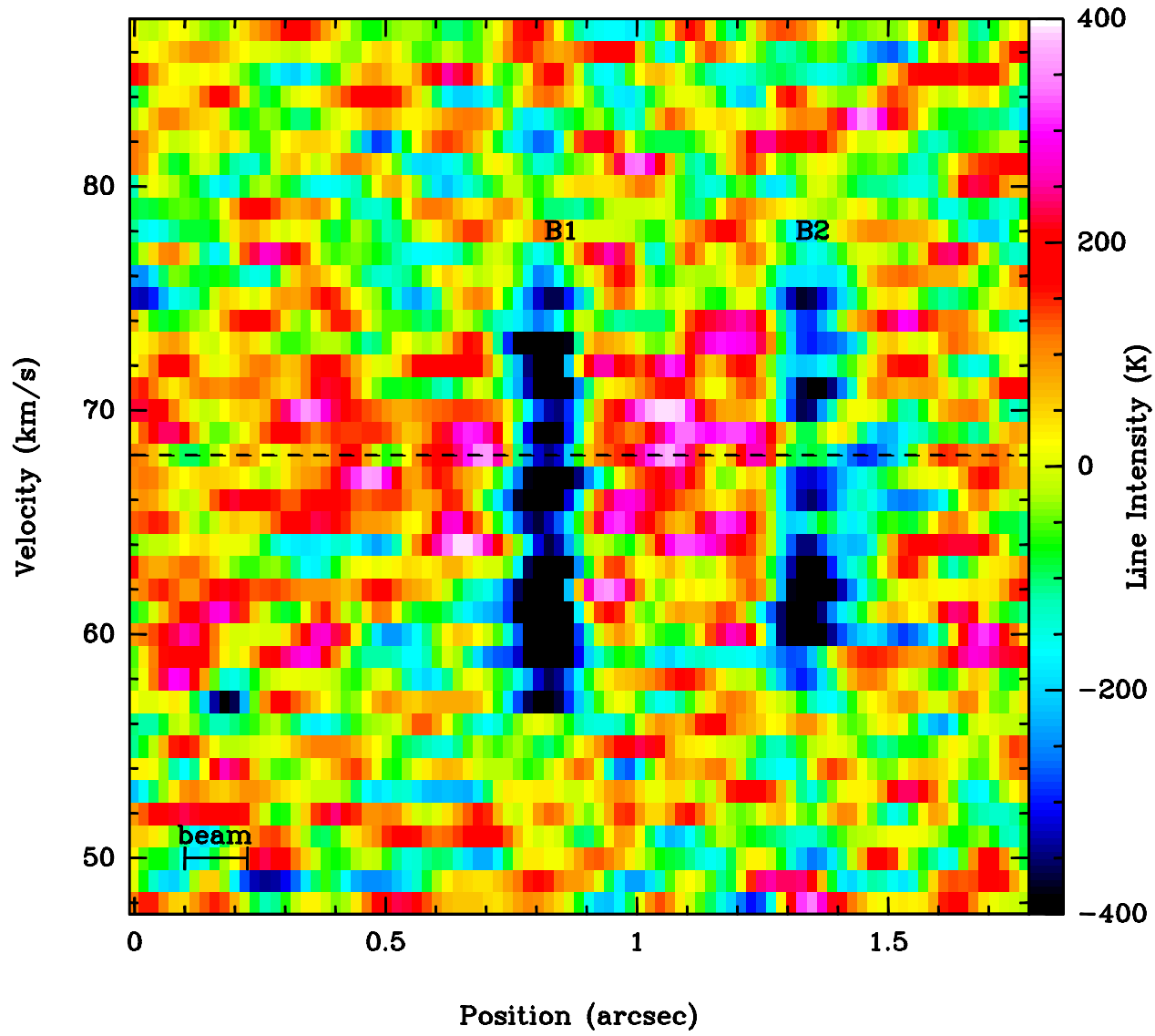

Figure 5.3: Position-velocity diagram of G10.47+0.03, along the arrow shown in Fig. 5.2. The velocity of 68 $\mathrm{km} \mathrm{s}^{-1}$ is marked as a dashed line, and the color scale is centered at $0 \mathrm{~K}$.

region of around $100 \mathrm{mJy}$ ), and a $10 \mathrm{mJy}$ point source at R.A. 17:47:19.905, Dec. -28:22:13.46 (4" north of K3). This latter source is associated with $\mathrm{C}_{2} \mathrm{H}_{5} \mathrm{CN}$ emission at $75 \mathrm{~km} \mathrm{~s}^{-1}$ and $3 \mathrm{~mm}$ continuum detected by Liu \& Snyder (1999). We note that emission from complex molecules (such as amino acetonitrile, Belloche et al. 2008) peaks at $\mathrm{K} 2$.

The integrated line map (Fig. 5.4 bottom) shows wide-spread line emission around K2. Toward K2 itself, the line absorbs over $80 \%$ of the continuum, implying an enormous optical depth (Fig. 5.1 and Table 5.2). The southern part seems to have a bit lower velocities than the northern part (Figs. 5.1 and 5.5).

\subsubsection{SgrB2-M}

The continuum map (Fig. 5.6 top) resembles the one obtained by de Pree et al. (1998). This source consists of a whole cluster of hypercompact HII regions, including F3 with $1.3 \mathrm{Jy}$ and F1c/e/f with about $0.5 \mathrm{Jy}$.

Line absorption is concentrated toward the strongest sources F1 and F3 (Fig. 5.6 bottom). Toward F1, the line is at $60 \mathrm{~km} \mathrm{~s}^{-1}$ with a component at $50 \mathrm{~km} \mathrm{~s}^{-1}$ in F1f (Fig. 5.7 top). A northeast-southwest velocity gradient from 69 to $73 \mathrm{~km} \mathrm{~s}^{-1}$ over $0.3^{\prime \prime}$ (2300 AU) is detected in F3 (Fig. 5.7 bottom). In addition to the lines from Fig. 5.1, there is a possible absorption line toward F2 at $57 \mathrm{~km} \mathrm{~s}^{-1}$ with a width of $7 \mathrm{~km} \mathrm{~s}^{-1}$ and a line-to-continuum ratio of 0.8. Although not visible in the integrated line map, there is also line emission present, as seen by averaging over a field outside the HII regions.

\subsubsection{Single-dish data}

We investigated several sources with the Effelsberg 100-m telescope for HCN direct $\ell$-type transitions, with no detections in $\mathrm{G} 31.41+0.31, \mathrm{G} 34.26+0.15, \mathrm{~W} 3\left(\mathrm{H}_{2} \mathrm{O}\right)$, and W51d. Toward W51e, the $J=12$ line was detected (with half the intensity of G10.47+0.03), but the lower- $J$ transitions are questionable ( $J=9$ at least 10 times lower than in G10.47+0.03). Clear detections in Galactic star-forming regions were only made in Orion-KL, G10.47+0.03, SgrB2-N, and -M. These latter three sources are shown in Fig. 5.8, with additional IRAM 30-m data for SgrB2. $J=19$ is contaminated by an $\mathrm{H} \gamma$ recombination line whose frequency corresponds to a $\sim 20 \mathrm{~km} \mathrm{~s}^{-1}$ higher velocity. $J=20$ is blended with a transition of ethanol in SgrB2-N and possibly with He $\gamma$ in -M. 


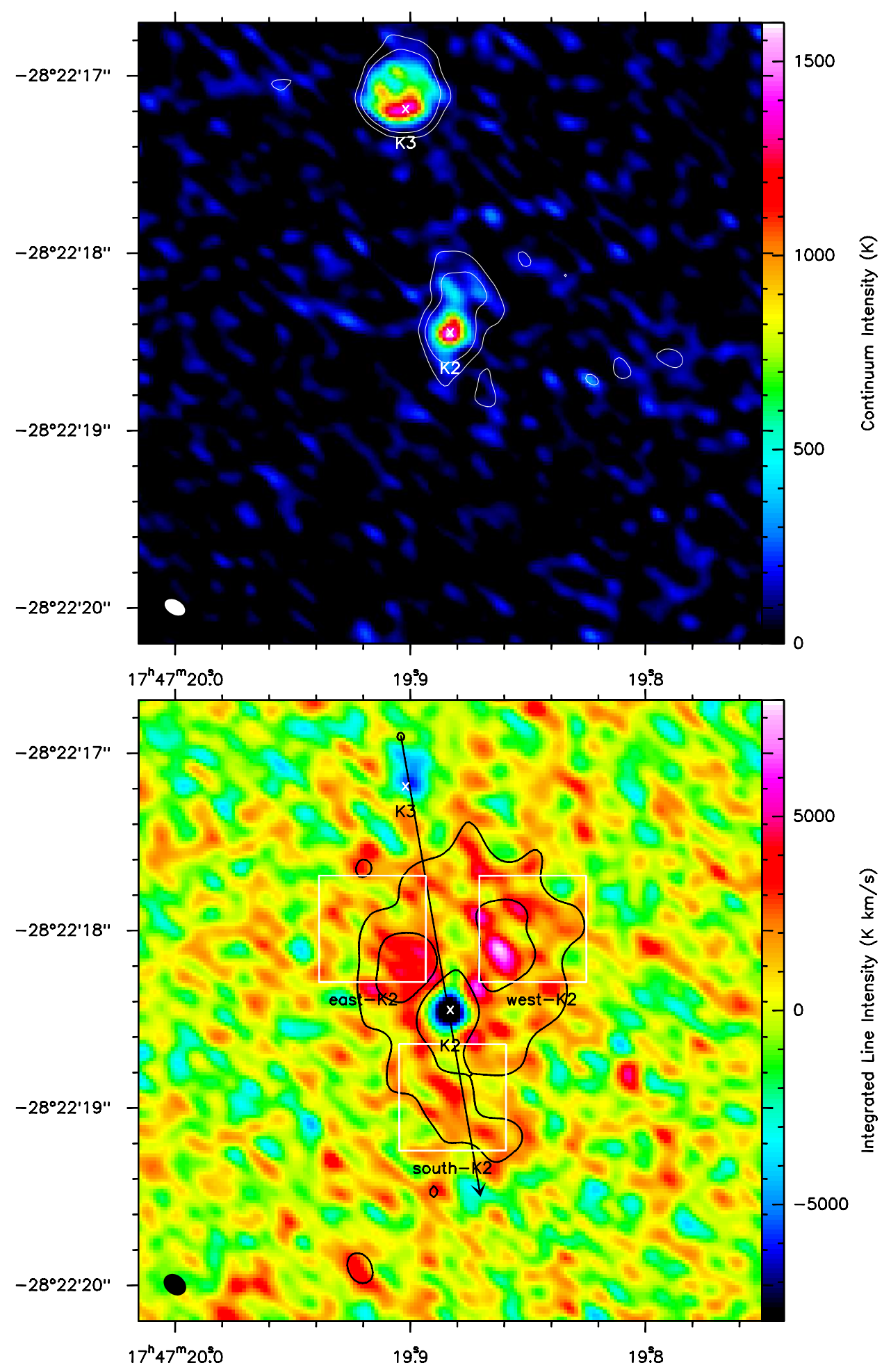

Figure 5.4: VLA maps of SgrB2-N. The 7mm continuum emission is shown on the top, the integrated line map on the bottom (beams are depicted in the lower left). Contours denote 3 and $6 \sigma$ levels of the naturally weighted continuum (white, top) and of the integrated line emission convolved to $0.25^{\prime \prime}$ resolution (black, bottom). The white crosses give the positions toward which the absorption spectra were obtained and the white boxes the area over which the emission spectra are integrated, shown in Fig. 5.1. The arrow denotes the direction along which the position-velocity plot of Fig. 5.5 was obtained. The map size is $3.5^{\prime \prime}$, about 0.13 pc. 


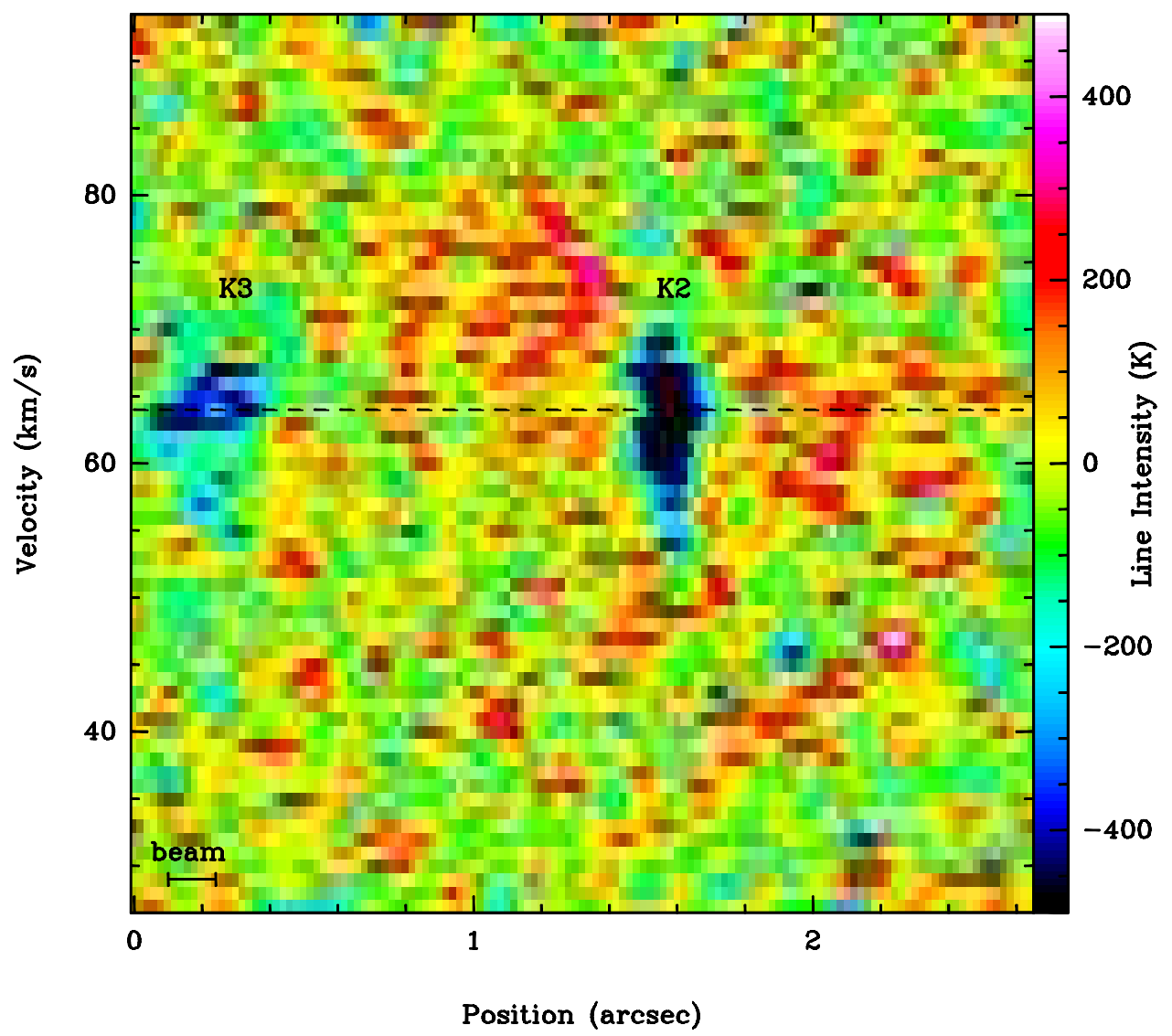

Figure 5.5: Position-velocity diagram of SgrB2-N, along the arrow shown in Fig. 5.4. The velocity of $64 \mathrm{~km} \mathrm{~s}^{-1}$ is marked as a dashed line, and the color scale is centered at $0 \mathrm{~K}$.

To compare our VLA data to the single-dish data, Fig. 5.9 shows the line integrated over the whole map of Figs. 5.2, 5.4, and 5.6. The VLA flux in G10.47+0.03 and SgrB2-N agrees well with the expectations from the Effelsberg data. In SgrB2-M, absorption dominates at low frequencies, but in the IRAM 30-m data weak emission can be seen. Emission also contributes to the VLA flux.

A bit puzzling is the different widths of $J=9$ and 10 in the Effelsberg data of SgrB2-M: It is $20 \mathrm{~km} \mathrm{~s}^{-1}$ in $J=9$ and only $6.6 \mathrm{~km} \mathrm{~s}^{-1}$ in $J=10$ (Fig. 5.8). As we found no blending at the $J=9$ frequency, this is probably due to different ratios of the 50,60, and $70 \mathrm{~km} \mathrm{~s}^{-1}$ components: For $J=10$, the $60 \mathrm{~km} \mathrm{~s}^{-1}$ component dominates.

In SgrB2-N, also the higher- $J$ transitions with level energies up to $2100 \mathrm{~K}$ were clearly detected. This allows to construct a rotation diagram (Fig. 5.10), yielding a temperature of $485 \pm 50 \mathrm{~K}$ and an $\mathrm{HCN}$ column density in a $1^{\prime \prime}$ source of $(5.8 \pm 2) \times 10^{19} \mathrm{~cm}^{-2}$. Note that taking absorption and optical depth into account would lead to a higher column density and a lower temperature, since both effects weaken especially the lower- $J$ lines.

\subsection{Modeling}

In an attempt to constrain the spatial structure of the hot molecular gas, we constructed radiative-transfer models that reproduce the observations. We employed a trial-and-error technique and compared the model to the data by eye. This method yields (at best) a model that is consistent with the data, but it cannot provide errors of the parameters, and different models could fit as well. The three-dimensional radiative-transfer code RADMC- $3 \mathrm{D}^{4}$ was used to compute the radiation that a model emits. It allows adaptive mesh refinement, following user-defined criteria. Dust properties and the dust density distribution as well as location, surface temperature and radius of stars are given as input. The program then computes the dust temperature by tracing photon packets which are randomly emitted by the stars. Additional inputs are density and temperature of ionized gas, the velocity field, fundamental molecular data, and the molecular abundance.

\footnotetext{
${ }^{4}$ http://www.ita.uni-heidelberg.de//dullemond/software/radmc-3d
} 


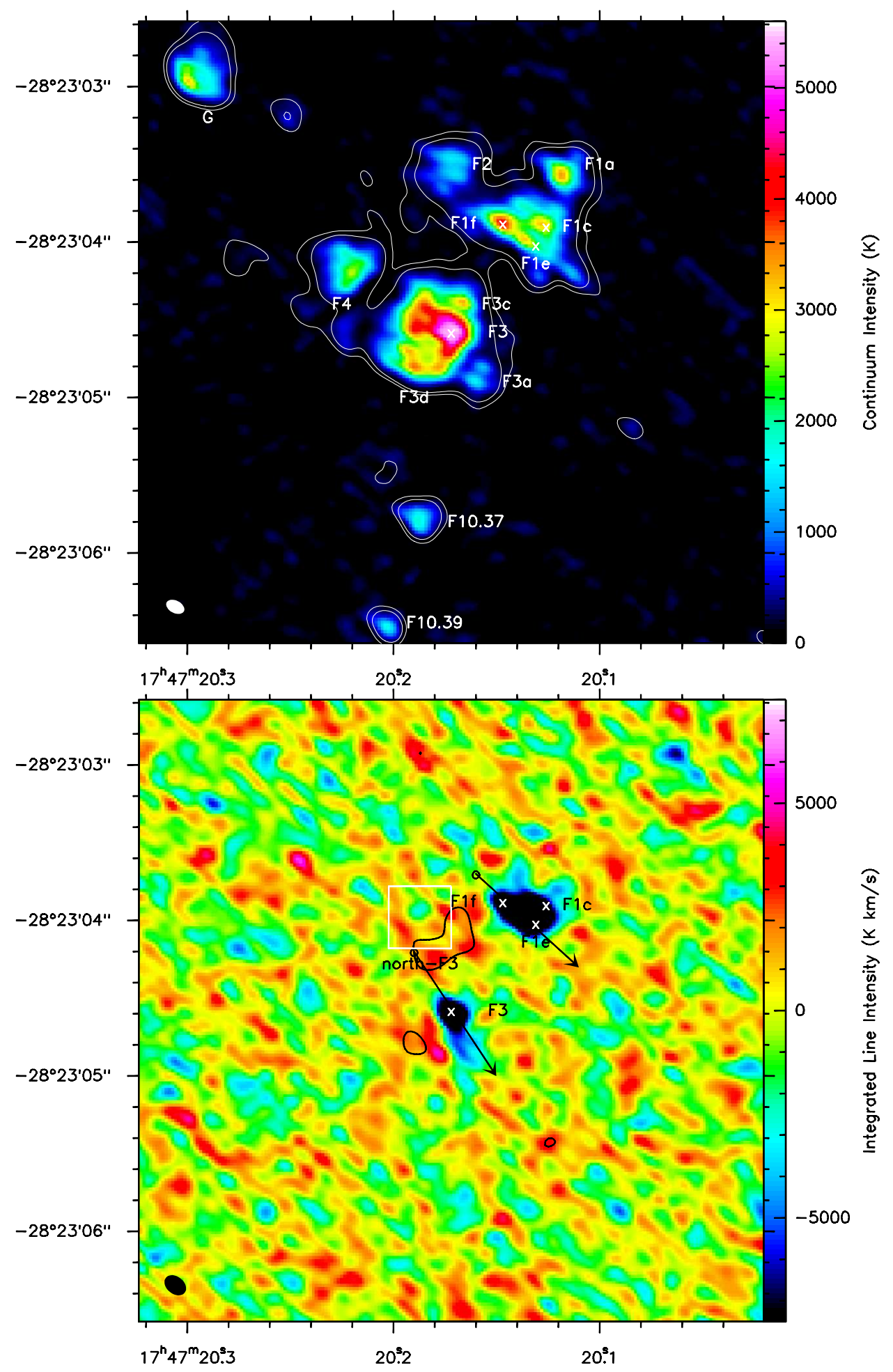

Figure 5.6: VLA maps of SgrB2-M. The 7mm continuum emission is shown on the top, the integrated line map on the bottom (beams are depicted in the lower left). Contours denote 3 and $6 \sigma$ levels of the naturally weighted continuum (white, top) and of the integrated line emission convolved to $0.25^{\prime \prime}$ resolution (black, bottom). The white crosses give the positions toward which the absorption spectra were obtained and the white boxes the area over which the emission spectra are integrated, shown in Fig. 5.1. The arrow denotes the direction along which the position-velocity plot of Fig. 5.7 was obtained. The map size is $4^{\prime \prime}$, about 0.15 pc. 

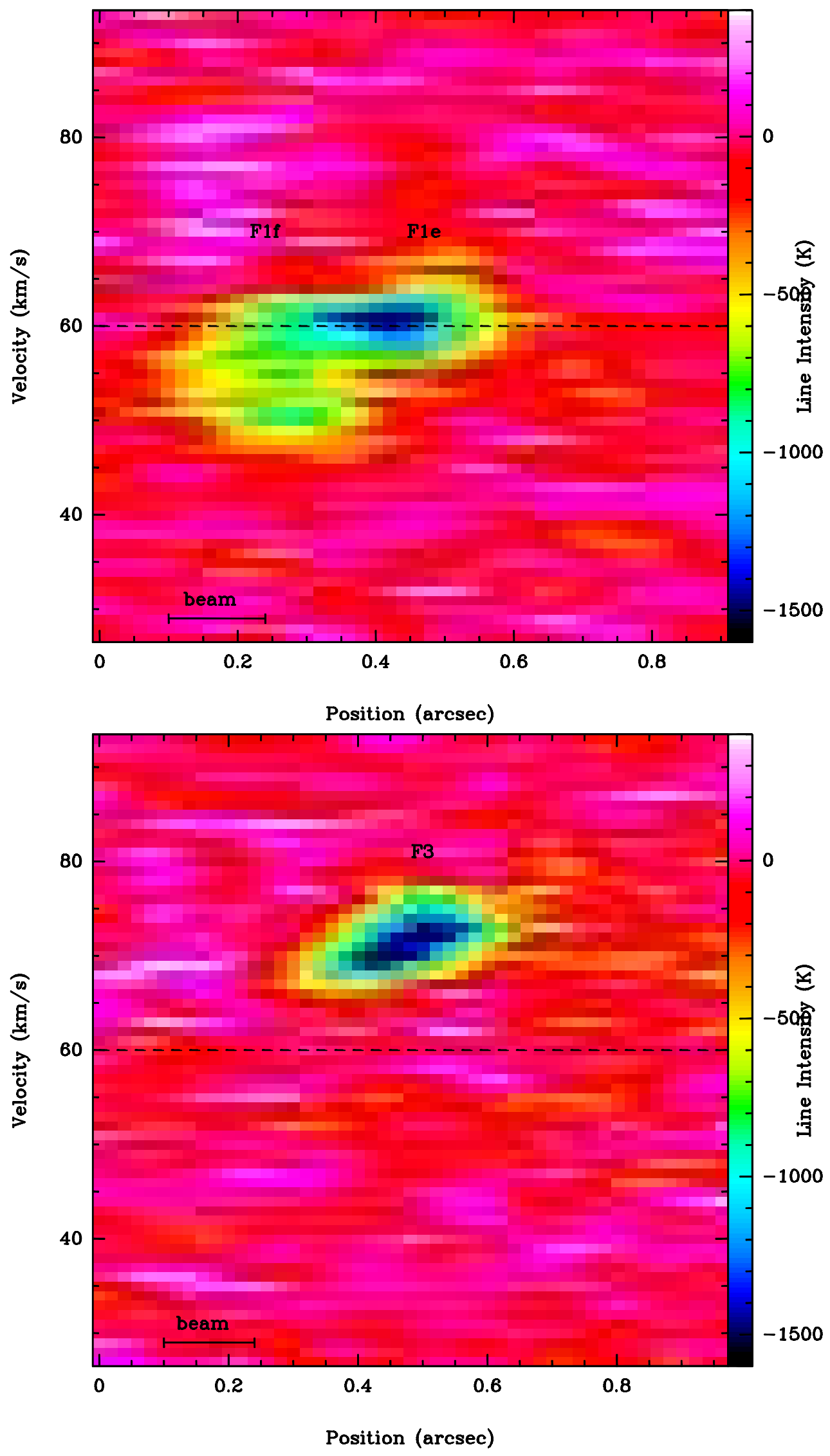

Figure 5.7: Position-velocity diagrams of SgrB2-M, along the arrows shown in Fig. 5.6. The cut through F1 is shown on the top, F3 on the bottom. The velocity of $60 \mathrm{~km} \mathrm{~s}^{-1}$ is marked as a dashed line, and the color scale is dominated by absorption. 

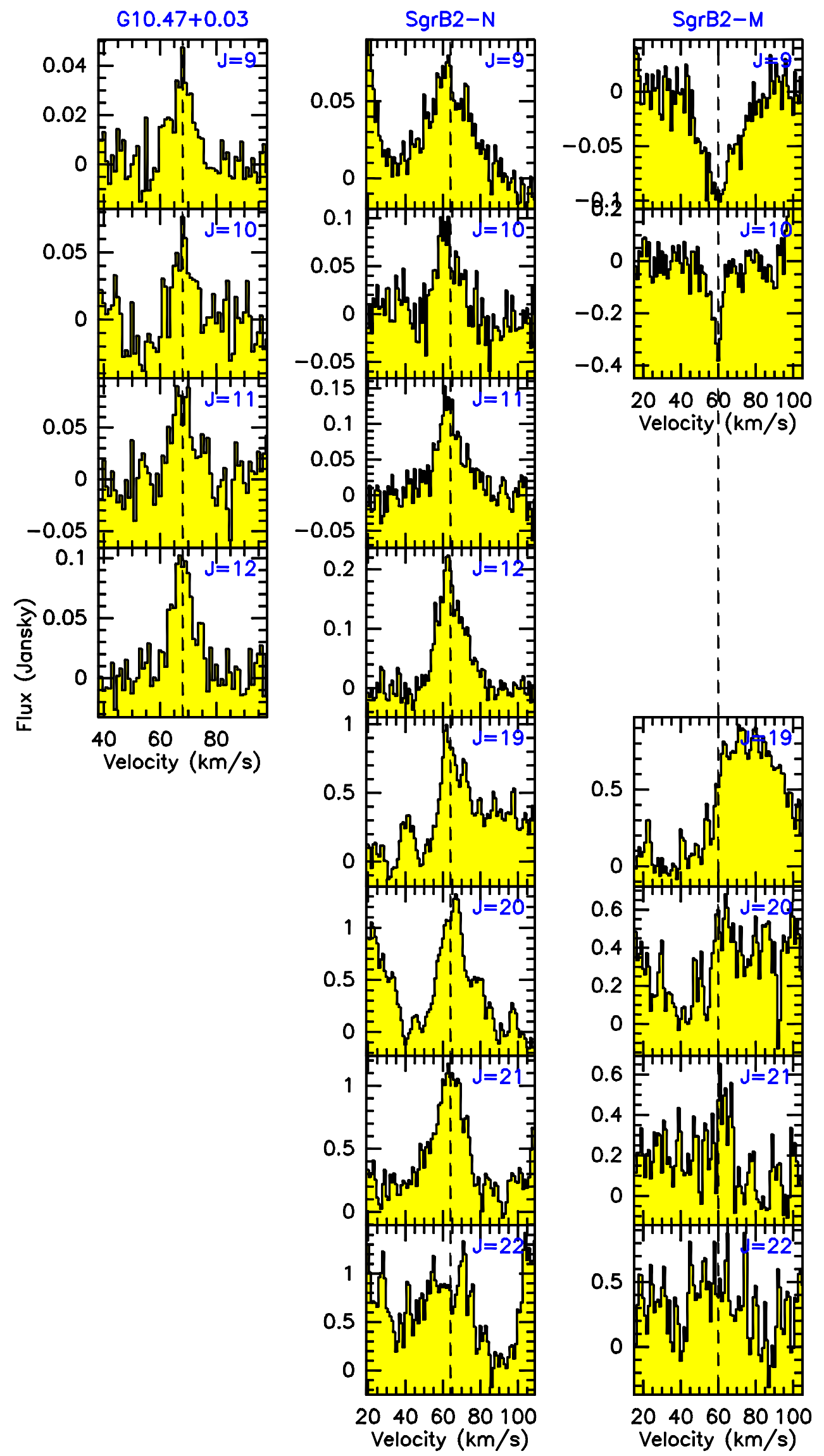

Figure 5.8: Single-dish spectra of G10.47+0.03 (left), SgrB2-N (central), and SgrB2-M (right). $J=9$ (20 GHz) to $12(35 \mathrm{GHz})$ were observed with the Effelsberg 100-m telescope, $J=19(85 \mathrm{GHz})$ to $22(113 \mathrm{GHz})$ with the IRAM $30-\mathrm{m}$ telescope. Beam sizes vary between 20 and $37^{\prime \prime}$, so the whole flux is received by the telescope. 

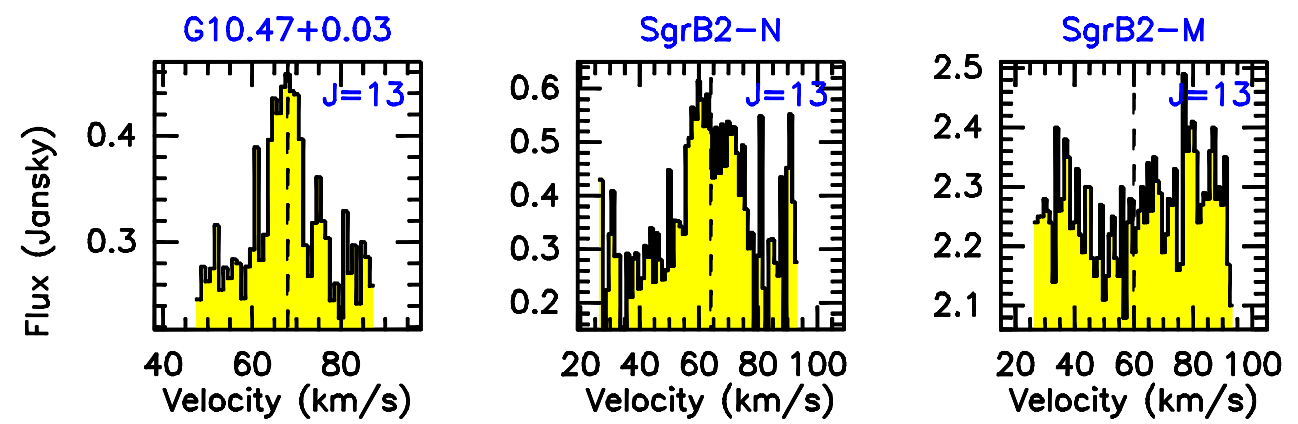

Figure 5.9: VLA line flux of G10.47+0.03 (left), SgrB2-N (central), and SgrB2-M (right), integrated over the whole maps shown in Figs. 5.2, 5.4, and 5.6.

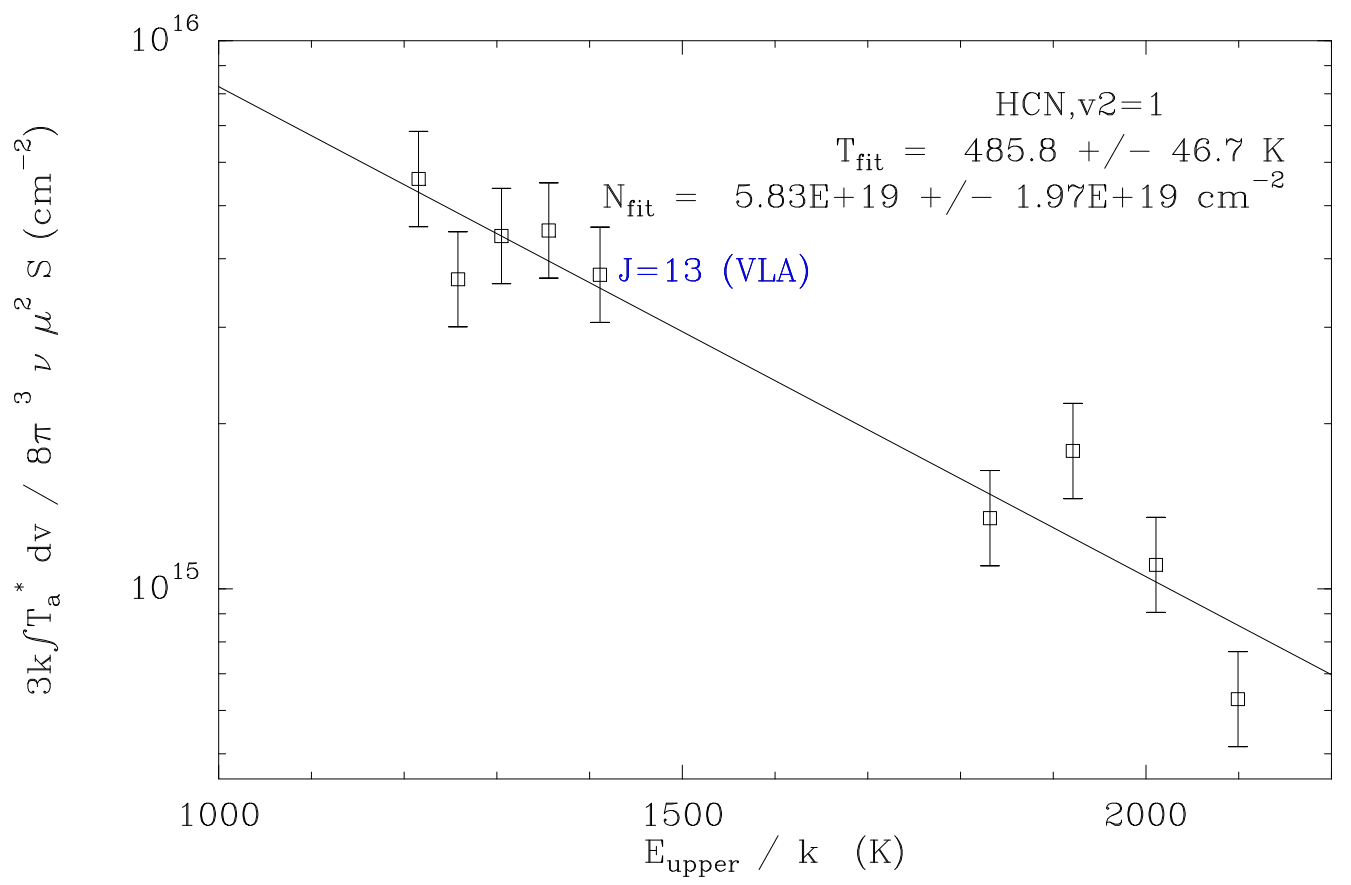

Figure 5.10: Rotation diagram for SgrB2-N, based on the single-dish fluxes. The integrated line flux is sourceaveraged for a source size of $1^{\prime \prime}$. The first four data points are from Effelsberg, the last four from IRAM 30-m; the VLA flux fits very well to the expectation.

\subsubsection{Assumptions}

We use the dust opacity from Ossenkopf \& Henning (1994) without grain mantles or coagulation. This is reasonable assuming that ice mantles around dust grains have evaporated completely and recently, so that the grains have had no time to coagulate again. Since the VLA continuum is dominated by free-free emission, the data are not sensitive to the dust emission and so we cannot constrain the dust optical depth, which is a combination of dust opacity and density, through the dust emission. However, the heating is strongly affected by the dust optical depth.

The temperature of the ionized gas is assumed to be $10^{4} \mathrm{~K}$, which is the order of magnitude expected from cooling by trace species. A different temperature would require a different electron density to account for the observed fluxes.

The HCN molecular data are from Thorwirth et al. (2003a) and taken from the Cologne Database for Molecular Spectroscopy (Müller et al. 2001, 2005). We use an $\mathrm{HCN}$ abundance of $10^{-5}$ relative to $\mathrm{H}_{2}$ for our models. Since we mainly constrain the density of $\mathrm{HCN}$, a different abundance would require different dust densities (with the gas/dust mass ratio of 100). Dust continuum data (from the SMA) indicate that the resulting dust densities are on the right order of magnitude, which justifies this abundance assumption.

The line radiative transfer assumes LTE (full non-LTE radiative transfer is also planned for RADMC-3D). LTE is a reasonable assumption for the observed line as HCN thermalizes to the ambient dust temperature: It is 
vibrationally excited by $14 \mu \mathrm{m}$ radiation emitted by warm dust, which is very optically thick at this wavelength due to the high densities (an optical depth of 1 is reached after about $25 \mathrm{AU}$ in a density of $10^{8} \mathrm{~cm}^{-3}$ ). In this warm and dense environment, also the levels in the ground vibrational state are thermalized by infrared pumping as well as by collisions and radiative excitation. Deviations from LTE occur at low temperatures, where the vibrational levels are not populated anyway.

The models have no macroscopic velocity field. We assume a constant intrinsic line width (microturbulence) throughout the source, which reflects internal motions. The resulting spectra are shifted by the source velocity $(68$ $\mathrm{km} \mathrm{s}^{-1}$ for G10.47+0.03, $64 \mathrm{~km} \mathrm{~s}^{-1}$ for SgrB2-N, $61 \mathrm{~km} \mathrm{~s}^{-1}$ for SgrB2-M F1e, and $71 \mathrm{~km} \mathrm{~s}^{-1}$ for SgrB2-M F3).

\subsubsection{Modeling results}

To compare the model to the observational data, the synthetic map produced by RADMC-3D, supplemented by distance and coordinate information, is Fourier-transformed, folded with the uv coverage of the observations, and imaged again. The first step of our fitting procedure is to reproduce the observed continuum by creating HII regions whose sizes and densities are based on Cesaroni et al. (2010) for G10.47+0.03 and de Pree et al. (1998) for SgrB2M. For SgrB2-N, we use similar models as for G10.47+0.03 since the $1.3 \mathrm{~cm}$ data of Gaume et al. (1995) are not sufficient in terms of angular resolution and wavelength. Sizes and densities of the HII regions are slightly modified to fit the observed continuum. The hypercompact HiI regions B1 and B2 in G10.47+0.03 and K2 in SgrB2-N have thus density gradients (with $r^{-1.5}$ and outer radii of $1590 \mathrm{AU}$ in B1, $950 \mathrm{AU}$ in B2 and $1000 \mathrm{AU}$ in $\mathrm{K} 2$ ), while all other HII regions have constant densities.

Cesaroni et al. (2010) and de Pree et al. (1998) also give spectral types of the exciting stars, which are converted to luminosity following Panagia (1973). These stars heat the dust and are, for simplicity, placed in the plane of the sky, except for two HII regions in SgrB2-M. The dust density distribution is adapted to fit the line observations. Figure 5.11 shows the column densities in $\mathrm{x}, \mathrm{y}$, and $\mathrm{z}$ directions of the three models that are described in the following.

G10.47+0.03 The model consists of a clump centered at B1 whose density follows a Gaussian with $7 \times 10^{7} \mathrm{H}_{2}$ $\mathrm{cm}^{-3}$ at the half-power radius of $7000 \mathrm{AU}$. This dust is heated by B1 with $10^{5} \mathrm{~L}_{\odot}, \mathrm{B} 2$ with $8.3 \times 10^{4} \mathrm{~L}_{\odot}$, and $\mathrm{A}$ with $4.6 \times 10^{4} \mathrm{~L}_{\odot}$. The intrinsic line width $(\mathrm{FWHM})$ is $8.3 \mathrm{~km} \mathrm{~s}^{-1}$. The model output is compared to the observations in Fig. 5.12.

SgrB2-N In the model for SgrB2-N, the dust is heated by $\mathrm{K} 2$ with $10^{5} \mathrm{~L}_{\odot}$ and $\mathrm{K} 3$ with $8.3 \times 10^{4} \mathrm{~L}_{\odot}$. The dust density follows a Gaussian centered at $\mathrm{K} 2$ with $7 \times 10^{7} \mathrm{H}_{2} \mathrm{~cm}^{-3}$ at the half-power radius of $10^{4} \mathrm{AU}$. An additional core (Gaussian with $5 \times 10^{8} \mathrm{H}_{2} \mathrm{~cm}^{-3}$ at the half-power radius of $1000 \mathrm{AU}$ ) $3000 \mathrm{AU}$ in front of $\mathrm{K} 2$ provides the strong absorption. The intrinsic line width is $10 \mathrm{~km} \mathrm{~s}^{-1}$. Figure 5.13 shows the comparison to the observations.

SgrB2-M In this model, the dust density follows two radial power laws with index 1.5, centered at F1f and F3. The first one starts with $5.5 \times 10^{8} \mathrm{H}_{2} \mathrm{~cm}^{-3}$ at a radius of $660 \mathrm{AU}$, the latter one with $5 \times 10^{8} \mathrm{H}_{2} \mathrm{~cm}^{-3}$ at $750 \mathrm{AU}$. Inside this latter radius, there is an HII region that contains a star of $8 \times 10^{4} \mathrm{~L}_{\odot}$. Additionally, a larger HII region is located $5000 \mathrm{AU}$ closer to us with a star of $2.5 \times 10^{5} \mathrm{~L}_{\odot}-\mathrm{F} 3$ is thus split into these two different HII regions. F1e with $2.5 \times 10^{4} \mathrm{~L}_{\odot}$ is located 2000 AU farther away from us than the other HII regions to facilitate absorption (Fig. 5.11). Further heating is provided by F1c, F1f, F2, F4 (each $\left.5.4 \times 10^{4} \mathrm{~L}_{\odot}\right), \mathrm{F} 1 \mathrm{a}\left(3.8 \times 10^{4} \mathrm{~L}_{\odot}\right)$, and F3a $\left(2.5 \times 10^{4} \mathrm{~L}_{\odot}\right)$. The intrinsic line width is $6.7 \mathrm{~km} \mathrm{~s}^{-1}$. A comparison to the observations is shown in Fig. 5.14.

These models are as simple as possible to approach a good fit to the observations, but are not unique. The model maps shown in Figs. 5.12 to 5.14 would appear more similar to the data if corresponding noise was added. This noise also makes it difficult to constrain more complicated models, since it is not clear how much of the clumpy substructure is real (which is on the order of only 2 or 3 times the rms noise)

\subsection{Discussion}

In this section, we discuss implications from the observational results and the modeling efforts.

\subsubsection{Optical depth}

As explained in Sect. 5.5, lower limits on optical depth and HCN column density can be derived from the absorption lines (Table 5.2). Outstanding is K2 in SgrB2-N, where the line is optically thick. The column densities of hot HCN toward the HII regions where it is detected are at least a few times $10^{19} \mathrm{~cm}^{-2}$, which translates into $\mathrm{H}_{2}$ column densities of $10^{24}$ to $10^{25} \mathrm{~cm}^{-2}$ even for a high HCN fractional abundance of $10^{-5}$. This represents only 

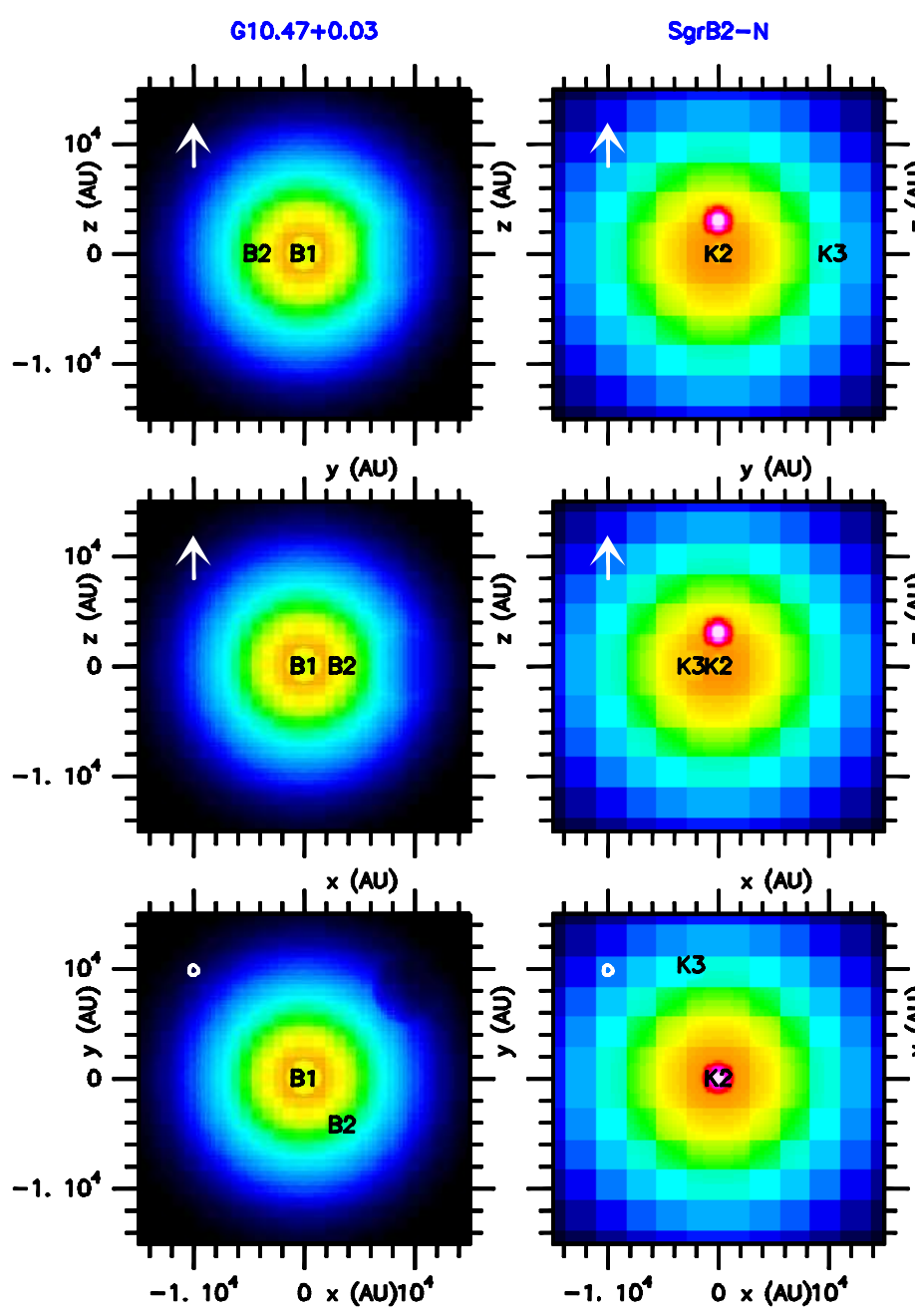

SgrB2-N

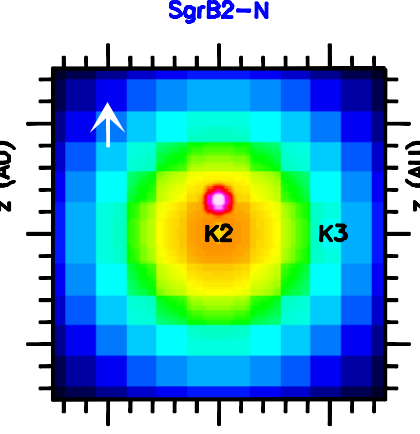

y (AU)

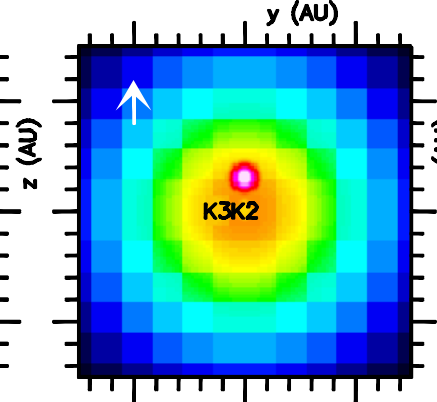

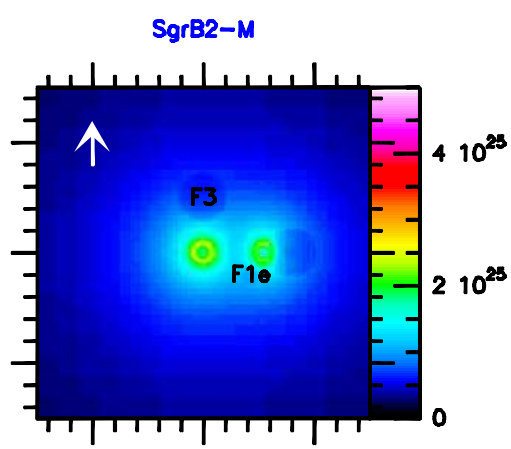

$y$ (AU)

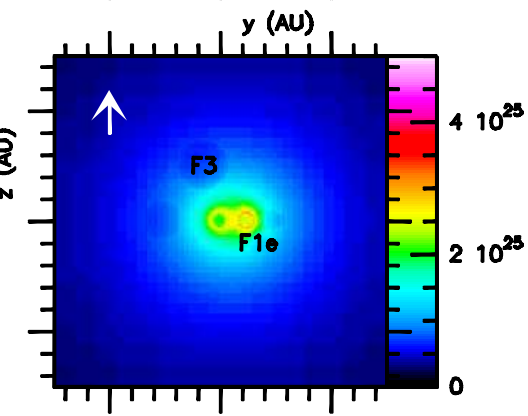

$\mathbf{x}(\mathrm{AU})$

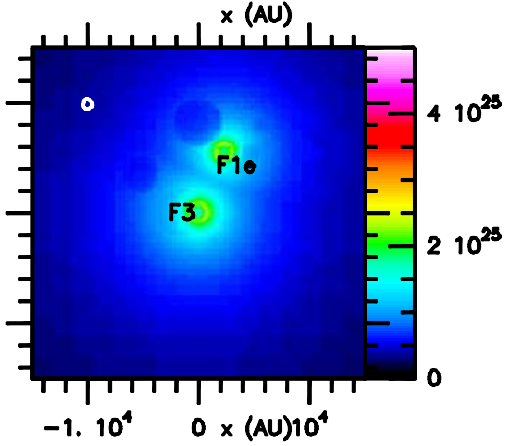

Figure 5.11: Column density maps of the models for the three sources. The lower panels show the column density (in $\mathrm{H}_{2} \mathrm{~cm}^{-2}$ ) in $\mathrm{z}$-direction, the central panels in $\mathrm{y}$-direction, and the upper panels in $\mathrm{x}$-direction (the arrow points to the observer, who is at positive $\mathrm{z}$ ). Two HII regions are marked for each source. The model radiation is compared to the observations in Figs. 5.12 to 5.14 .

the molecular gas at temperatures above roughly $300 \mathrm{~K}$. In the models, the mass above $300 \mathrm{~K}$ is $350 \mathrm{M}_{\odot}$ in G10.47+0.03, $500 \mathrm{M}_{\odot}$ in SgrB2-N, and $200 \mathrm{M}_{\odot}$ in SgrB2-M.

Such large quantities of hot molecular gas are necessary to explain the observations, and challenge theories of massive star formation. Current hydrodynamical simulations produce far too little hot molecular gas (e.g. Peters et al. 2010, with a total mass of $1000 \mathrm{M}_{\odot}$ ), both absolute and relative to the total mass, since the highly inhomogeneous structure (filamentary disks) does not lead to diffusion of the heating radiation. This is probably due to the lower initial mass and hence lower column densities and dust optical depths of these models, and it is to be hoped that in the future simulations of cores as massive as the ones presented in the current paper will reproduce our results. Also a high abundance of $\mathrm{HCN}$ (on the order of $10^{-5}$ ) in the dense, warm gas is needed, since the dust densities of our models are consistent with dust continuum data from the SMA and a lower abundance would mean an even higher mass of the hot gas. We expect future chemical models to compute such a high HCN abundance, probably through high-temperature gas-phase reactions.

Although HCN thermalizes to the ambient dust temperature, the population difference between the two $\ell$-type levels is very small, making them sensitive to the exact excitation conditions. The line optical depth is proportional to the population difference,

$$
\tau \propto N_{1}-N_{\mathrm{u}} \propto e^{-\frac{1411 \mathrm{~K}}{T_{\mathrm{l}}}}-e^{-\frac{1413 \mathrm{~K}}{T_{\mathrm{u}}}}
$$

where $T_{1}$ and $T_{\mathrm{u}}$ are the excitation temperatures for the lower and upper state, respectively. A difference of $0.1 \%$ between $T_{1}$ and $T_{\mathrm{u}}$ causes $\tau$ to change by more than $70 \%$; inversion occurs if $T_{\mathrm{u}}$ is $0.14 \%$ larger than $T_{1}$. The two levels are independently coupled to the ground vibrational state through the $14 \mu \mathrm{m}$ transitions, which determine the excitation temperatures. If these are different, the analysis would be far more difficult, as one would have to know the exact $14 \mu \mathrm{m}$ radiation field, where lines could slightly affect the optical depths. However, there is no such 

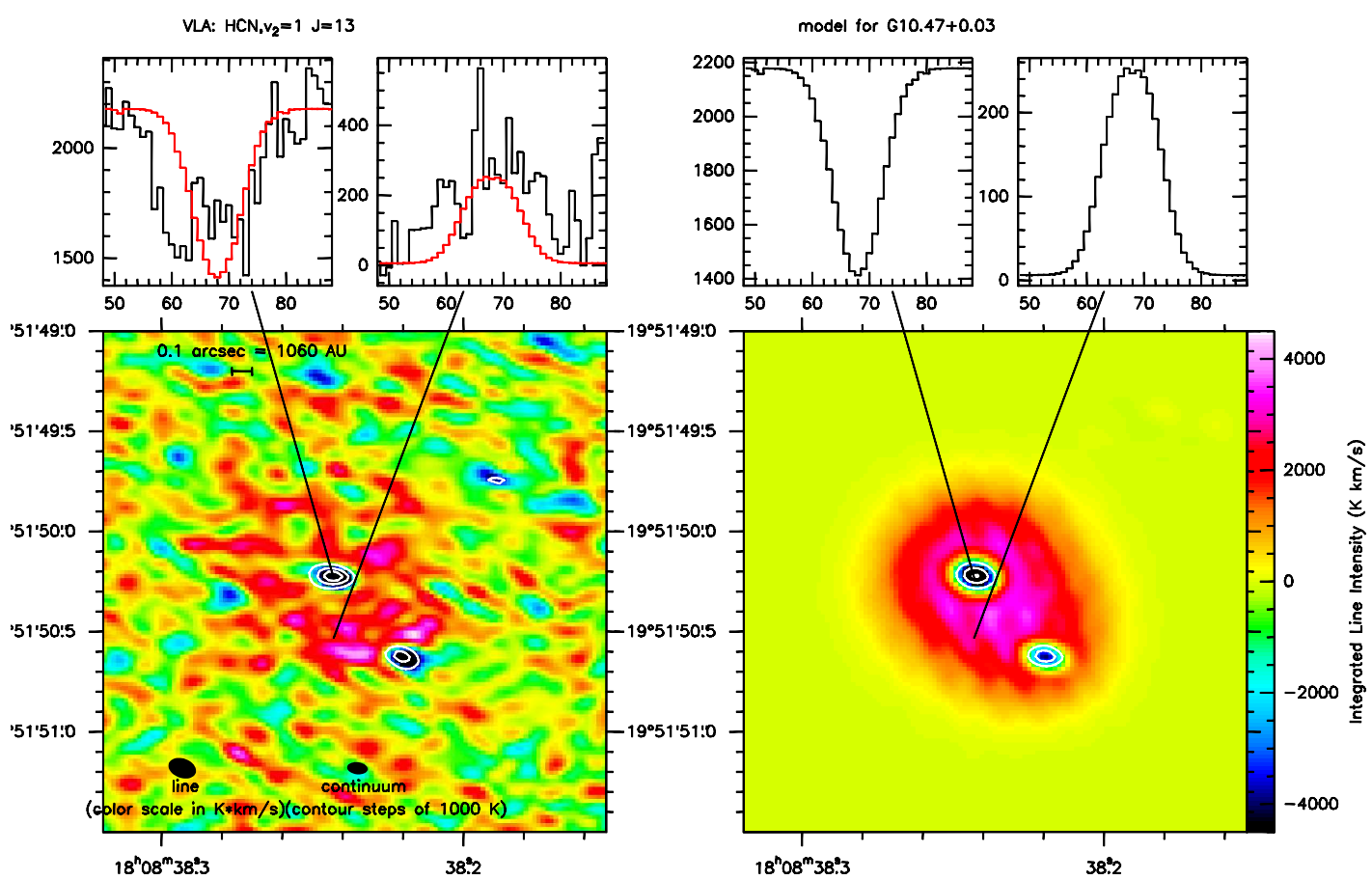

Figure 5.12: Model for G10.47+0.03 (right) compared to the data (left). The model spectra are also overlaid in red. The white contours denote the continuum in steps of $1000 \mathrm{~K}$.
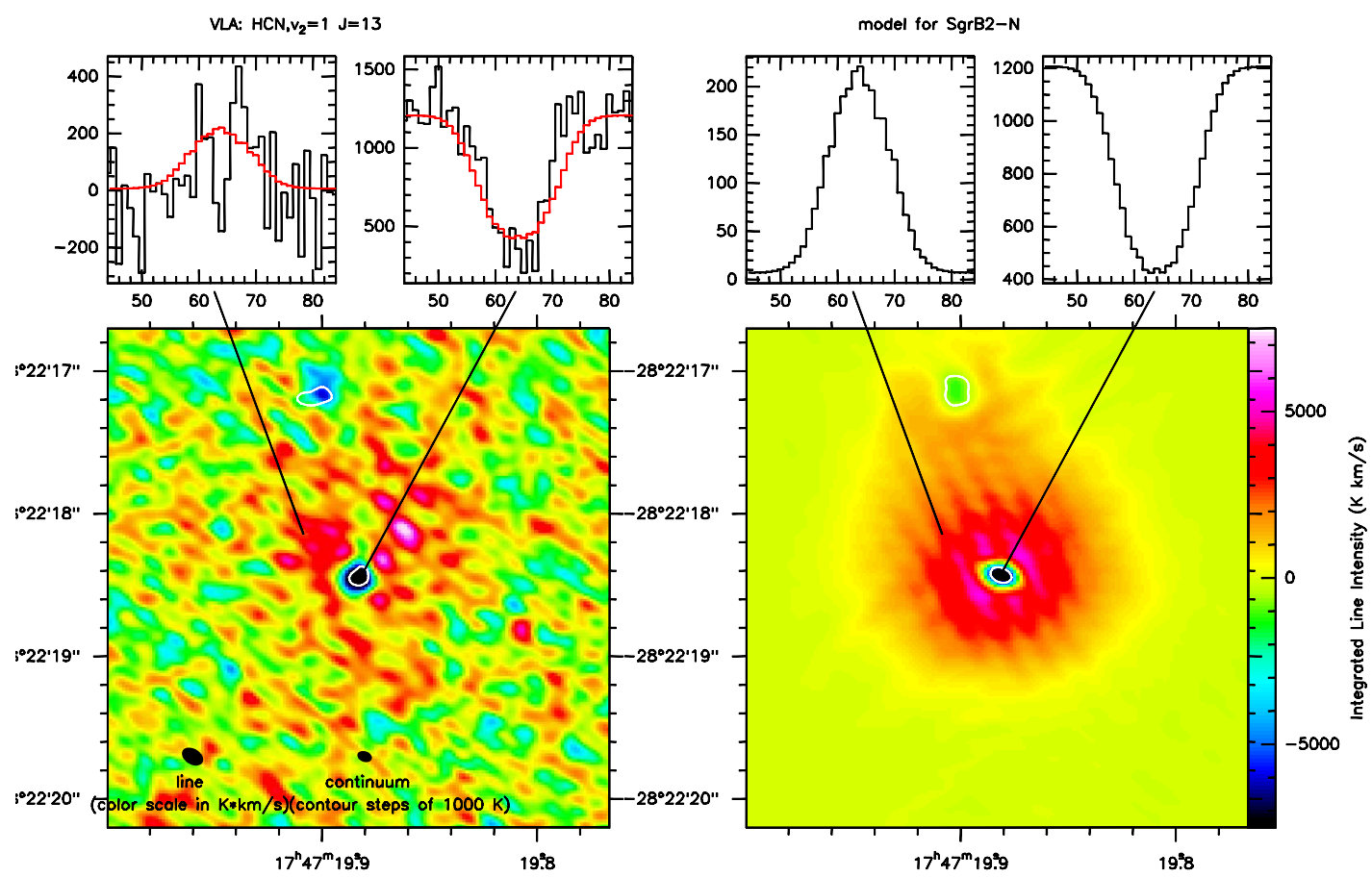

Figure 5.13: Model for SgrB2-N (right) compared to the data (left). The model spectra are also overlaid in red. The white contours denote the continuum in steps of $1000 \mathrm{~K}$. 


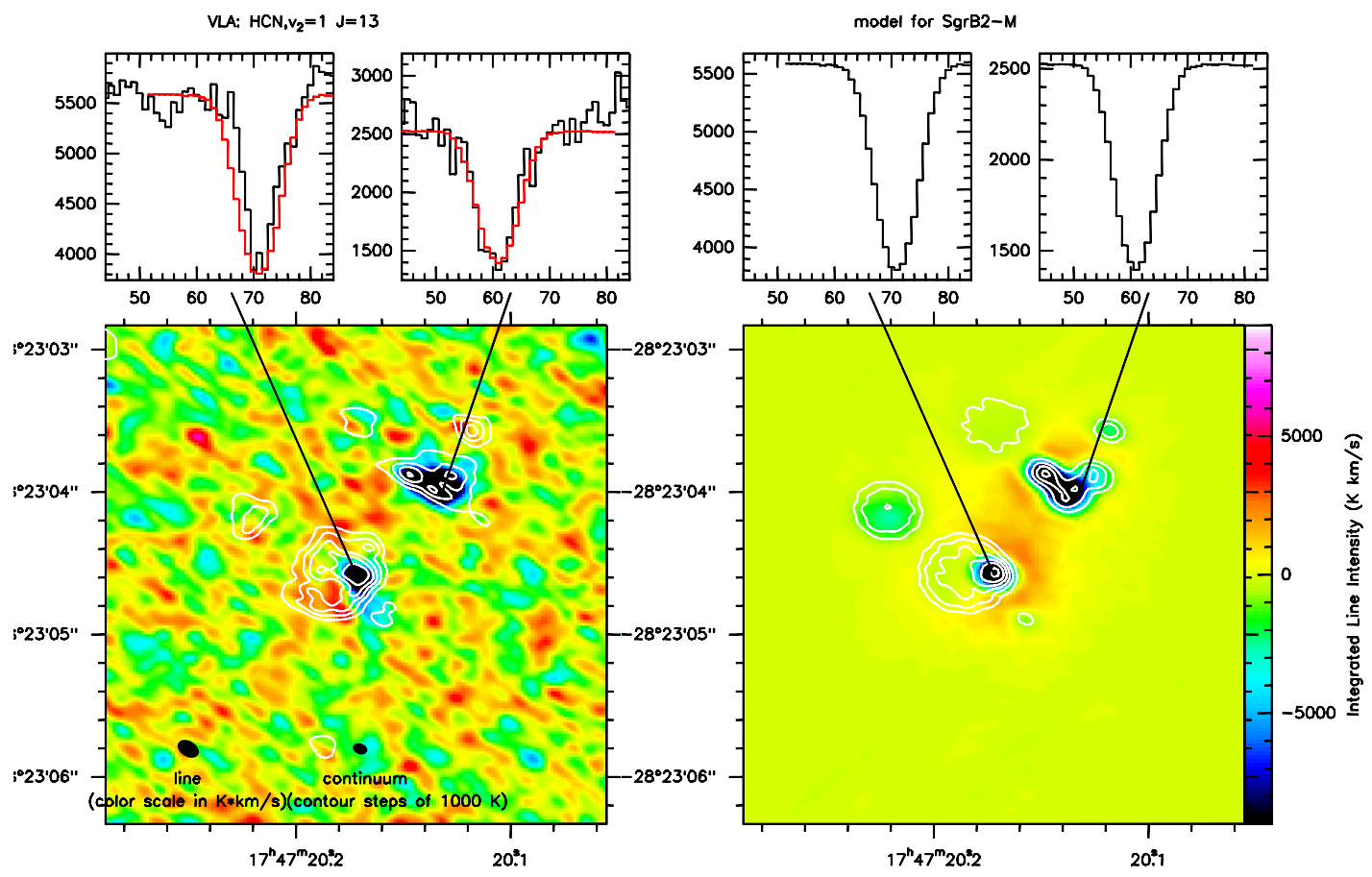

Figure 5.14: Model for SgrB2-M (right) compared to the data (left). The model spectra are also overlaid in red. The white contours denote the continuum in steps of $1000 \mathrm{~K}$.

mechanism known, and an extreme sub-thermal population difference, which would greatly reduce the required absorption column densities, is highly unlikely because of the agreement between absorption and emission, between $J=13$ and other direct $\ell$-type lines (Fig. 5.10), and between the derived column densities and dust emission data. Therefore, we consider LTE a good assumption for this transition.

If we assume a similar optical depth for the emission lines, say 0.3 , and a temperature of $400 \mathrm{~K}$, then the line intensity should be $100 \mathrm{~K}$, which is just the noise level in $1 \mathrm{~km} \mathrm{~s}^{-1}$ channels and $0.13^{\prime \prime}$ beams. Owing to the strength of the free-free radiation, we are thus more sensitive to absorption than emission lines. Averaging over a larger region (Fig. 5.1 and Table 5.3) lowers both noise and peak intensities. The latter reach up to $300 \mathrm{~K}$ in G10.47+0.03, suggesting that optical depth and temperature are higher than the above mentioned assumptions. Surprising is the detection of the line toward G10.47+0.03 A, since no $\mathrm{NH}_{3}(4,4)$ satellites were detected by Cesaroni et al. (2010).

In the model for SgrB2-M, we have placed the Hir regions F3 and F1e in front of and behind the bulk of the molecular gas, respectively. This prevents absorption toward most of F3 and leads to stronger absorption toward F1e. The exact offsets in $\mathrm{z}$ are not well constrained, however. To obtain the very strong absorption toward K2 in SgrB2-N, we put a very dense core just in front of the HII region. This is of course not very satisfying as it is unlikely that such a core is exactly along the line-of-sight. It was however not possible to reproduce both emission and absorption with a more symmetric distribution.

\subsubsection{Heating}

Heating up large masses of molecular gas requires deeply embedded massive (proto)stars with high luminosities. Their radiation is either originally in the infrared (Hosokawa \& Omukai 2009), not producing HII regions, or is quickly processed to the infrared by dust absorption of the UV radiation. Due to high column densities in all directions, the dust is optically thick even in the infrared and this radiation cannot escape, but diffuses outwards by multiple absorption/emission events until the dust is optically thin to its own radiation. This diffusion (or radiative trapping) leads to much higher temperatures in the inner part; e.g. at $2000 \mathrm{AU}$ from the HCHII region B1, diffusion raises the temperature from $150 \mathrm{~K}$ in a low-density model, where diffusion is not effective, to $450 \mathrm{~K}$ in the highdensity model for G10.47+0.03. This mechanism therefore seems indispensable to account for large masses of hot gas. Additionally, by absorbing stellar UV radiation the dust protects the molecules from dissociation. In general, heating is more efficient if the heating source is inside a density condensation (Kaufman et al. 1998) than if it is external, as could be the case for G34.26+0.15 (Watt \& Mundy 1999; Mookerjea et al. 2007).

In our models, the diffusion mechanism is accomplished by dense spherical clumps around heating sources. It 
is however not clear how many heating sources there are: The stars in the HII regions are sufficient to reproduce the observed line strength, as the presented models demonstrate. But a good fit can also be achieved when lowerluminosity stars are added to the models according to the Initial Luminosity Function, without changing the stars in the HII regions. So on the basis of these data it seems not possible to distinguish between the scenarios of heating only by the stars ionizing the HII regions and heating by HII regions plus embedded sources which do not emit in the radio regime. We may speculate that there are much more stars present than detected from the free-free radiation, since the observed sources are clusters in formation. Also, there could be more extended (faint) line emission (and the absorption coming from a larger column) than in the models, which would point to additional heating sources. Furthermore, the simple models predict strong self-absorption in the (optically thick) submm rotational transitions within the $v_{2}=1$ state of $\mathrm{HCN}$ and $\mathrm{H}^{13} \mathrm{CN}$, which is however not observed with the SMA, presumably due to clumpiness (Rolffs et al. 2011, in prep.).

While not excluding the possibility of additional heating sources, we could show that heating by the stars in the hypercompact HII regions is sufficient to reproduce the observations. Hence, our modeling results suggest that it is unlikely that the main heating is due to embedded objects without ionized gas. Given that the sources are dense condensations deeply embedded in giant molecular clouds and have high column densities, diffusion of radiation, which is necessary to increase the heating, is reasonable even for more inhomogeneous and asymmetric structures than modeled.

\subsubsection{Velocity field}

Many systematic motions are hidden in the line width of around $10 \mathrm{~km} \mathrm{~s}^{-1}$, which contains both turbulent and macroscopic motions. These components (e.g. the presence of rotation, infall, or outflows) can not be disentangled. For all emission lines, the signal-to-noise ratio is too poor to gain accurate information on the velocity structure.

G10.47+0.03 The most striking feature in this source is an additional blue-shifted absorption component in both B1 and B2 at a velocity of around $60 \mathrm{~km} \mathrm{~s}^{-1}$, compared to the source velocity of $68 \mathrm{~km} \mathrm{~s}^{-1}$ (Fig 5.1). The $\mathrm{NH}_{3}(4,4)$ absorption (Cesaroni et al. 1998, 2010) is at $53 \mathrm{~km} \mathrm{~s}^{-1}$ toward B1 and at $61 \mathrm{~km} \mathrm{~s}^{-1}$ toward B2, with much lower optical depth than the emission component. An explanation could be that the colder gas, probed by $\mathrm{NH}_{3}(4,4)$ with its level energy of $200 \mathrm{~K}$, is mainly in an outflow cone, while the hot gas probed by $\mathrm{HCN}, v_{2}=1, J=13$ is mainly at the systemic velocity close to the HCHII regions and only partly expanding. With the SMA, we see broad blueshifted absorption in several lines toward the dust continuum peak between B1 and B2 (Rolffs et al., in prep.). This indicates expansion motions involving many different molecular excitation conditions, while at the same time large-scale infall is present (Rolffs et al. 2011b). Given the high excitation and the different velocities, an unrelated foreground velocity component is unlikely. The region west of B1 appears to move at a velocity of $67 \mathrm{~km} \mathrm{~s}^{-1}$, while south of $\mathrm{B} 1$ the velocity is $69 \mathrm{~km} \mathrm{~s}^{-1}$.

SgrB2-N While the strong absorption line toward $\mathrm{K} 2$ has a line width of $10 \mathrm{~km} \mathrm{~s}^{-1}$ at the source velocity of $64 \mathrm{~km} \mathrm{~s}^{-1}$, the emission lines north of $\mathrm{K} 2$ have a higher line width and higher velocity, possibly indicating an additional component at $\sim 70 \mathrm{~km} \mathrm{~s}^{-1}$.

SgrB2-M Three velocity components can be seen from the absorption lines (Fig. 5.7), one at around $50 \mathrm{~km} \mathrm{~s}^{-1}$ in F1f, one at $60 \mathrm{~km} \mathrm{~s}^{-1}$ in F1c, F1e, and F1f, and one at around $70 \mathrm{~km} \mathrm{~s}^{-1}$ in F3. Relative to the ambient velocity of 60 or $64 \mathrm{~km} / \mathrm{s}$, which itself is not well determined due to different components, the F1f line shows blue-shifted absorption, indicating an expansion component, and the F3 line is red-shifted. This could mean infall or a motion of the whole F3 core. The F3 line shows a northeast-southwest velocity gradient from 69 to $73 \mathrm{~km} \mathrm{~s}^{-1}$ over $0.3^{\prime \prime}$ (2300 AU). If that is interpreted as Keplerian rotation, and if it is assumed that the whole orbit is seen and is edge-on, the lower limit on the central mass would be $5 \mathrm{M}_{\odot}$. The large-scale velocity field shows expansion in the inner parts and infall in the outer parts (Rolffs et al. 2010).

\subsection{Conclusions}

With the VLA, we have obtained maps of a high-excitation $(1400 \mathrm{~K}) \mathrm{HCN}$ line at high angular resolution $\left(0.1^{\prime \prime}\right)$ toward three massive star-forming regions. We see both absorption toward small and dense HII regions and emission. The infered optical depths require on the order of $10^{24} \mathrm{H}_{2} \mathrm{~cm}^{-2}$ and 100 solar masses of hot $(>300 \mathrm{~K})$ molecular gas within a region of less than $0.1 \mathrm{pc}$. We note that the column densities of hot HCN derived from the absorption lines are only lower limits, and can be substantially higher especially due to beam dilution. Heating is provided by the stars powering hypercompact HII regions, possibly complemented by heating sources invisible in the radio domain. Diffusion of radiation in an optically thick dusty environment is likely to increase the temperatures. The 
line velocities reveal expansion motions in G10.47+0.03 and components at 50, 60, and $70 \mathrm{~km} \mathrm{~s}^{-1}$ in SgrB2-M, the latter with a velocity gradient. Our three-dimensional radiative transfer modeling with RADMC-3D demonstrate the great power and potential of our approach to constrain the source structure of high-mass star-forming regions. HCN direct $\ell$-type lines are a unique tool, whose future observation will also be possible with ALMA and the SKA.

\section{Acknowledgements}

We thank the VLA staff for having carried out the observations. ST acknowlegdes support by the Deutsche Forschungsgemeinschaft through grant TH 1301/3-1. 


\section{Chapter 6}

\section{SMA paper: Structure of the Hot Molecular Core G10.47+0.03}

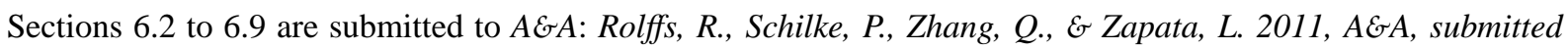
(Rolffs et al. 2011c, received 20 April 2011)

\subsection{Overview}

Telescope: The SMA (Submillimeter Array) is a submillimeter interferometer atop Mauna Kea, Hawaii (4200m altitude). It consists of eight 6-m dishes. A spectrally resolved bandwidth of 2 times $2 \mathrm{GHz}$ (new: $4 \mathrm{GHz}$ ), separated by $10 \mathrm{GHz}$, can be provided for most of the frequencies between 180 and $700 \mathrm{GHz}$. The SMA is operated by the Harvard-Smithsonian Center for Astrophysics (CfA) in Cambridge, MA, and the Academia Sinica Institute of Astronomy and Astrophysics in Taiwan.

Observations: Observations of the massive hot molecular core G10.47+0.03 started in 2007 in the extended configuration at $345 / 355 \mathrm{GHz}$, followed by $690 \mathrm{GHz}$ observations in 2008, aided by a near location of Jupiter, whose moons can serve as calibrator sources. The angular resolution of the $345 / 355 \mathrm{GHz}$ data was improved by the very extended configuration in 2009.

Modeling: The modeling was done in a similar way to the VLA data, using RADMC-3D. The continuum is dust emission, which constrains the density distribution. The line data are much more complicated than in the case of the VLA data, however. As the lines from the ground vibrational state would require non-LTE radiative transfer, only vibrationally excited HCN was modeled.

Results: Absorption features show the presence of an outflow. The density has a central flattening, reminiscent of a Plummer profile. Lines from vibrationally excited HCN are very optically thick, but not self-absorbed. The modeling suggests that this is due to clumpiness and the non-spherical velocity field.

\subsection{Abstract}

Context: The physical structure of Hot Molecular Cores, where forming massive stars have heated up dense dust and gas, but have not yet ionized the molecules, poses a prominent challenge in the research of high-mass star formation and astrochemistry.

Aims: We aim at constraining the spatial distribution of density, temperature, velocity field, and chemical abundances in the Hot Molecular Core G10.47+0.03.

Methods: With the Submillimeter Array (SMA), we obtained high spatial and spectral resolution of a multitude of molecular lines at different frequencies, including at $690 \mathrm{GHz}$. At $345 \mathrm{GHz}$, our beam size is $0.3^{\prime \prime}$, corresponding to $3000 \mathrm{AU}$. We analyze the data using the three-dimensional dust and line radiative transfer code RADMC-3D, and myXCLASS for line identification. 
Table 6.1: Observational summary

\begin{tabular}{lcccccc}
\hline \hline Date & $\begin{array}{c}\text { frequency } \\
(\mathrm{GHz})\end{array}$ & array configuration & $\begin{array}{c}\text { baseline }^{a} \\
(\mathrm{~m})\end{array}$ & no. antennas & $\begin{array}{c}\text { integration time } \\
(\mathrm{min})\end{array}$ & gain calibrator \\
\hline 12 Aug 2007 & $345 / 355$ & extended & 205 & 7 & 281 & $1733-130$ \\
10 May 2008 & $681 / 691+201 / 211$ & compact north & 125 & 5 & 270 & Callisto \\
6 Sep 2008 & $681 / 691+345 / 355$ & subcompact & 70 & 6 & 82 & Callisto \\
7 Sep 2008 & $681 / 691+345 / 355$ & subcompact & 70 & 7 & 93 & Callisto, Ganymede \\
4 July 2009 & $345 / 355$ & very extended & 516 & 8 & 330 & $1733-130$ \\
\hline
\end{tabular}

Notes. ${ }^{(a)}$ Maximum projected baseline

Results: We find hundreds of molecular lines from complex molecules and high excitations. Even vibrationally excited $\mathrm{HC}^{15} \mathrm{~N}$ at $690 \mathrm{GHz}$ is detected. Absorption against the dust continuum occurs in twelve transitions, whose shape implies an outflow along the line-of-sight. Outside the continuum peak, the line shapes are indicative of infall. Dust continuum and molecular line emission are resolved at $345 / 355 \mathrm{GHz}$, revealing central flattening and rapid radial falloff of the density outwards of $10^{4} \mathrm{AU}$, best reproduced by a Plummer radial profile of the density. No fragmentation is detected, but modeling of the line shapes of vibrationally excited HCN suggests the density to be clumpy.

Conclusions: We conclude that G10.47+0.03 is characterized by beginning of feedback from massive stars, while infall is ongoing. Large gas masses (hundreds of $\mathbf{M}_{\odot}$ ) are heated to high temperatures, aided by diffusion of radiation in a high-column-density environment. The increased thermal, radiative, turbulent, and wind-driven pressure drives expansion in the central region and is likely responsible for the central flattening of the density.

\subsection{Introduction}

Massive stars and star clusters are born deeply embedded in molecular clouds (for a review, see Zinnecker \& Yorke 2007). When cores have sufficiently contracted to form massive stars, the dense dust and gas is heated by these stars. The ice mantles around dust grains evaporate, and a rich plethora of molecular lines can be observed, along with strong dust emission (Kurtz et al. 2000; Cesaroni 2005). Often, these Hot Molecular Cores are associated with ultracompact or hypercompact HII regions, which are ionized by newly formed massive stars (Hoare et al. 2007). The physical and chemical structure of Hot Molecular Cores is of great importance for the study of high-mass star formation and of astrochemistry. Investigations of the structure are hampered, though, by the compactness of the sources, by their scarcity and large distance, and by the large foreground column density, which only longwavelength radiation can pass.

What is needed, hence, is high angular resolution at (sub)millimeter wavelenghts, combined with high spectral resolution of many molecular lines, which contain all the information about chemistry, velocity field, and temperature. With the Submillimeter Array (SMA) in Hawaii, we observed the massive Hot Molecular Core G10.47+0.03 at around 200, 345, and $690 \mathrm{GHz}$, yielding a best resolution of $0.3^{\prime \prime}$ and 350 identified molecular lines. Due to its large dust mass and high temperatures, this hot core is among the strongest sources at submm wavelengths, although it is located at a distance of $10.6 \mathrm{kpc}$ (Pandian et al. 2008). It has an estimated luminosity of $7 \times 10^{5} \mathrm{~L}_{\odot}$ (Cesaroni et al. 2010) and displays exceptionally many lines from highly excited molecules (e.g. $\mathrm{HC}_{3} \mathrm{~N}$, Wyrowski et al. 1999).

\subsection{Observations and data reduction}

The high-mass star-forming region G10.47+0.03 was observed with the Submillimeter Array ${ }^{1}$ (SMA) during five nights in different array and receiver configurations, yielding six two-GHz-wide bands centered at around 201, 211, $345,355,681$, and $691 \mathrm{GHz}$. Table 6.1 summarizes the observations. We note that Jupiter was close $\left(\sim 10^{\circ}\right)$ to the target source in 2008, so its moon Callisto could be used as gain calibrator at $690 \mathrm{GHz}$, where it is otherwise very difficult to find a suitable calibrator source. It was observed repeatedly for 2 minutes after spending 10 minutes on the target source. For the $345 \mathrm{GHz}$ observations in 2007 and 2009, after each 15 minutes we switched to 1733-130 (NRAO 530), which lies $10.9^{\circ}$ away from the target source. In addition, strong sources (Uranus, Ceres, 3c454.3, $3 \mathrm{c} 273$ ) were observed before and after these loops to allow bandpass and flux calibration.

\footnotetext{
${ }^{1}$ The Submillimeter Array is a joint project between the Smithsonian Astrophysical Observatory and the Academia Sinica Institute of Astronomy and Astrophysics and is funded by the Smithsonian Institution and the Academia Sinica (Ho et al. 2004).
} 

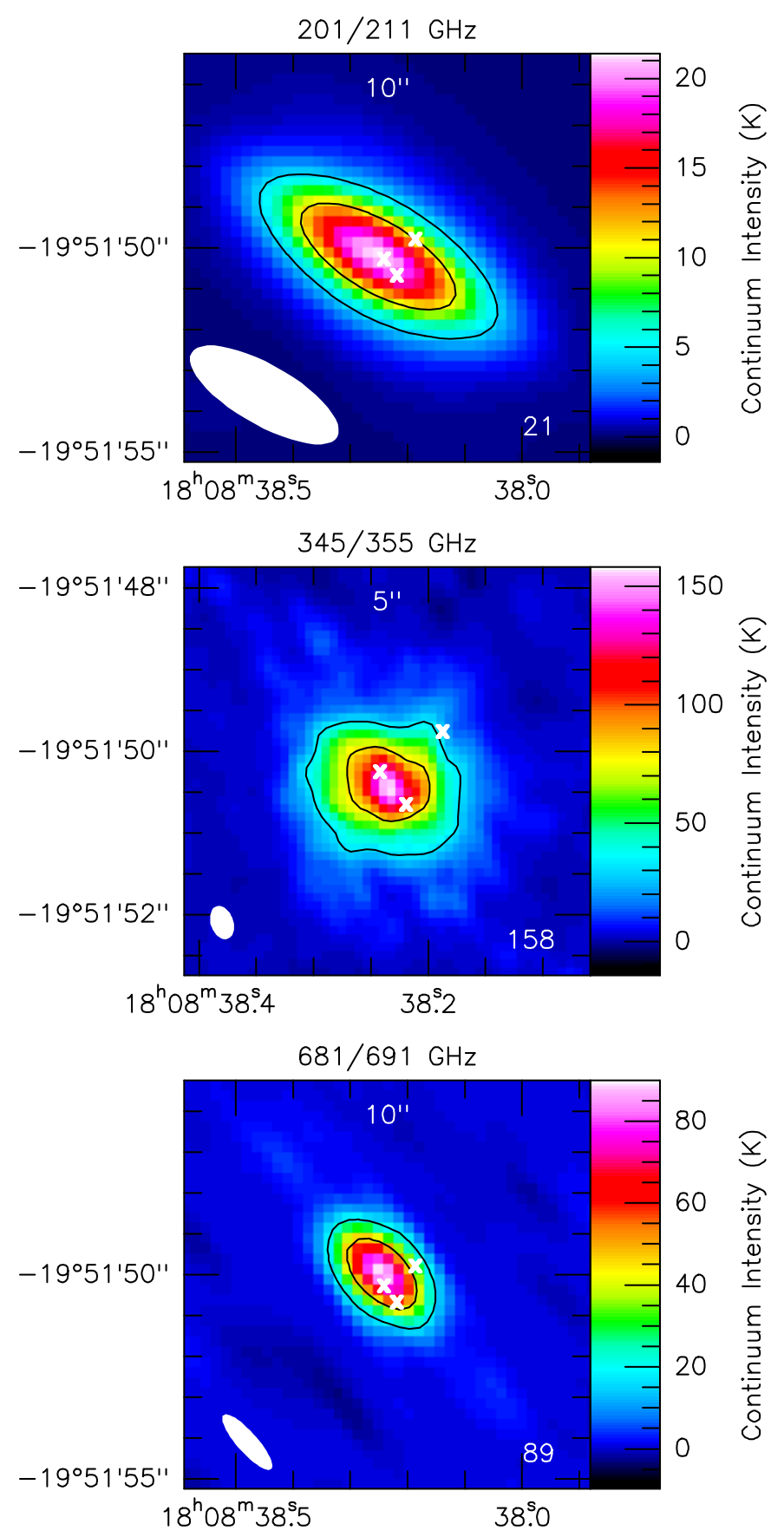

Figure 6.1: Continuum maps of G10.47+0.03 observed with the SMA at 201/211 GHz (top panel), $345 / 355 \mathrm{GHz}$ (central), and $681 / 691 \mathrm{GHz}$ (bottom). The map size is either 5 or $10^{\prime \prime}$. Contours mark 20 and $50 \%$ of the peak flux, which is given in the lower right (in K). The beam is depicted in the lower left. The white crosses denote the HII regions $\mathrm{B} 1, \mathrm{~B} 2$, and $\mathrm{A}$ (from left to right). 
Table 6.2: Beam sizes and noise levels

\begin{tabular}{lccc}
\hline $\begin{array}{l}\text { Frequency } \\
(\mathrm{GHz})\end{array}$ & $\begin{array}{c}\text { beam }^{a} \\
\left({ }^{\prime \prime} ;{ }^{\circ}\right)\end{array}$ & $\begin{array}{c}\mathrm{rms}^{b} \\
(\mathrm{mJy} / \text { Beam })\end{array}$ & $(\mathrm{K})$ \\
\hline $199.9-201.8$ & $4.27 \times 1.84 ; 57$ & 38 & 0.15 \\
$209.9-211.8$ & $4.14 \times 1.95 ; 55$ & 37 & 0.13 \\
$201 / 211$ cont. & $4.14 \times 1.48 ; 60$ & 2.9 & 0.014 \\
\hline $344.1-345.9$ & $0.45 \times 0.29 ; 18$ & 55 & 4.3 \\
$354.1-355.9$ & $0.42 \times 0.29 ; 22$ & 55 & 4.4 \\
$345 / 355$ cont. & $0.42 \times 0.28 ; 20$ & 12 & 1.0 \\
\hline $680.3-682.2$ & $2.12 \times 0.65 ; 37$ & 840 & 1.6 \\
$690.3-692.2$ & $2.08 \times 0.60 ; 38$ & 820 & 1.7 \\
$681 / 691$ cont. & $1.77 \times 0.50 ; 42$ & 227 & 0.67 \\
\hline
\end{tabular}

Notes. (a) Different weighting schemes were used for continuum and line images. Major and minor axis and position angle (from north to east) are given. ${ }^{(b)} \mathrm{rms}$ noise is given for a frequency width of $1 \mathrm{~km} / \mathrm{s}$ in the line maps. It is evaluated from -10 to $-5^{\prime \prime}$ in both RA and Dec relative to the phase center.

Calibration and editing of the data were done in the IDL MIR package ${ }^{2}$, involving correction and application of the system temperature, phase-only bandpass calibration, continuum regeneration, phase-and-amplitude bandpass calibration, flagging, gain calibration (phase and amplitude), and flux calibration. The data were converted to MIRIAD $^{3}$ (Sault et al. 1995), where the edge channels of each chunk were flagged and the channels were rebinned to a width of $1 \mathrm{~km} \mathrm{~s}^{-1}$ at $201 \mathrm{GHz}, 0.75 \mathrm{~km} \mathrm{~s}^{-1}$ at $345 \mathrm{GHz}$, and $1.42 \mathrm{~km} \mathrm{~s}^{-1}$ at $681 / 691 \mathrm{GHz}$. The different dates were merged for each frequency setup. Since the 2007 observations had the phase center at R.A. 18:08:38.28, Dec. -19:51:50.0, while the later observations were centered on R.A. 18:08:38.232, Dec. $-19: 51: 50.4$, the 345/355 GHz data set had to be merged in AIPS. The channels less affected by spectral lines were identified and used for separating lines and continuum in MIRIAD. The continuum data of upper and lower sideband were merged and imaged using almost uniform weighting at $201 / 211 \mathrm{GHz}$ and $345 / 355 \mathrm{GHz}$ (robust= -2 ) and more natural weighting at $681 / 691 \mathrm{GHz}$ (robust=0.5). A cutoff for cleaning of about 3 times the rms noise in the image was used. Based on these clean components, the continuum visibility data were self-calibrated, which substantially reduced the fluctuations outside the source, and the solutions were applied to the line data as well. Imaging of the spectrally resolved data was done with robust $=0.5$ at $201 / 211 \mathrm{GHz}$ and $345 / 355 \mathrm{GHz}$ and robust $=2$ at $681 / 691 \mathrm{GHz}$. Table 6.2 gives the resulting beam sizes and noise levels in the maps.

Line identification was made with the myXCLASS program ${ }^{4}$, which accesses the CDMS 5 and JPL ${ }^{6}$ molecular $^{5}$ data bases. All figures in this paper were made with the GILDAS software ${ }^{7}$.

\subsection{Observational results}

\subsubsection{Continuum}

Figure 6.1 shows the obtained continuum maps. The total flux is $6 \mathrm{Jy}$ at $201 / 211 \mathrm{GHz}, 27 \mathrm{Jy}$ at $345 / 355 \mathrm{GHz}$, and $95 \mathrm{Jy}$ at $681 / 691 \mathrm{GHz}$, corresponding to a spectral index of 2.8 between the lower two and 1.8 between the upper two frequencies. While the beam sizes are not sufficient to resolve the continuum emission at 201/211 and $681 / 691 \mathrm{GHz}$, the extension can be clearly seen at $345 / 355 \mathrm{GHz}$. At this frequency, the peak intensity is $158 \mathrm{~K}$ (1.84 Jy/Beam), and the $3 \sigma$ contour extends over $3^{\prime \prime}$.

\subsubsection{Line identification}

The data cubes were convolved to a common resolution of $5^{\prime \prime}$ and central spectra were extracted. This resolution was chosen to decrease the noise and the effects of a strong continuum, as well as to facilitate a comparison between the different frequencies; it gives the total (source-integrated) flux for most lines.

To identify the spectral features, a simple homogeneous model was computed in Local Thermodynamic Equlibrium (LTE), using the myXCLASS program. A source size of $1.5^{\prime \prime}$, a temperature of $200 \mathrm{~K}$ and a line width of 5

\footnotetext{
${ }^{2}$ http://www.cfa.harvard.edu/cqi/mircook.html

${ }^{3} \mathrm{http}: / /$ bima.astro.umd.edu/miriad

${ }^{4}$ https://www.astro.uni-koeln.de/projects/schilke/XCLASS

${ }^{5} \mathrm{http}: / /$ www.cdms.de

${ }^{6}$ http://spec.jpl.nasa.gov

${ }^{7}$ http://www.iram.fr/IRAMFR/GILDAS
} 

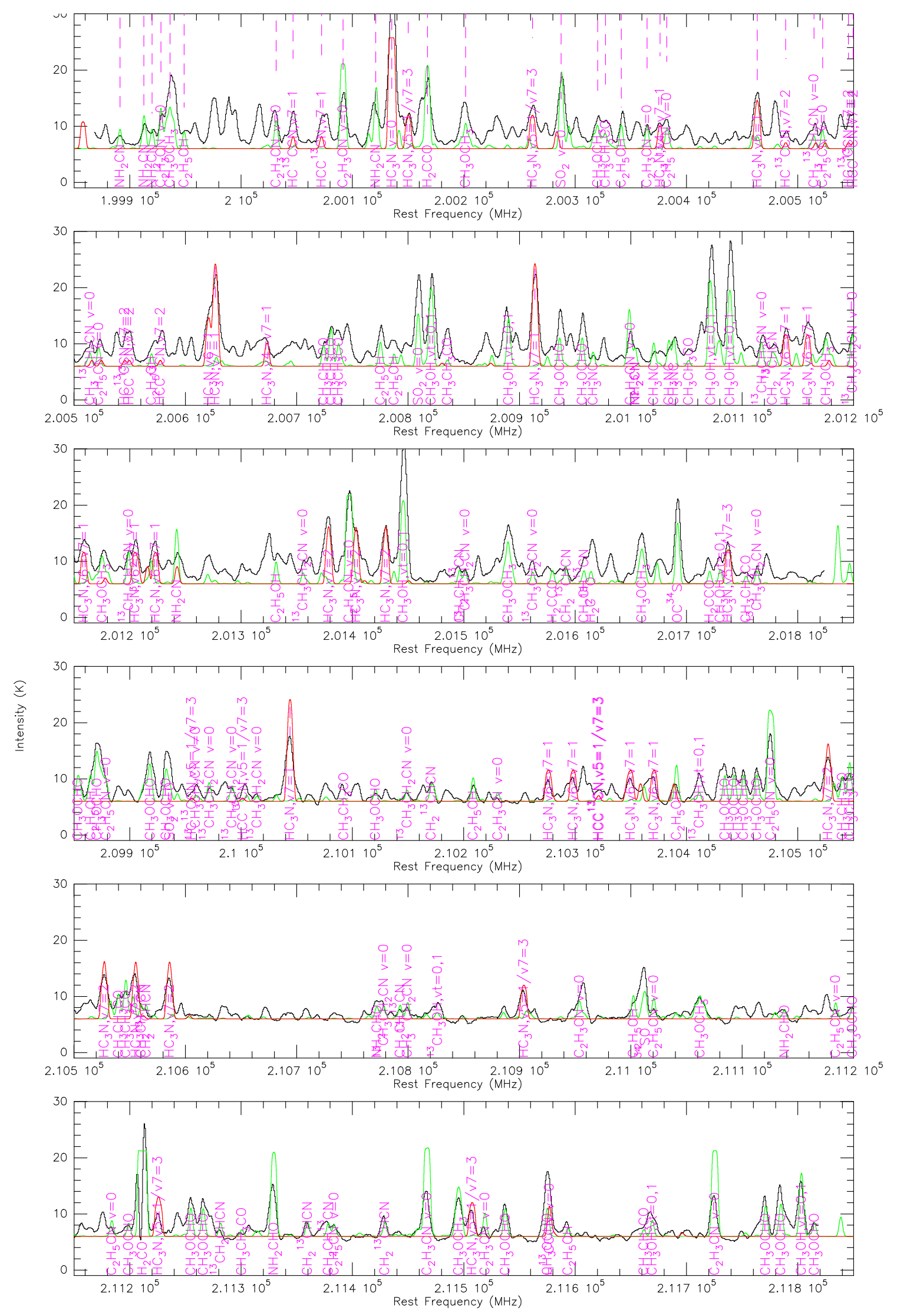

Figure 6.2: $201 / 211 \mathrm{GHz}$ spectral range convolved to $5^{\prime \prime}$ resolution. The green overlay is the model for all molecules except $\mathrm{HC}_{3} \mathrm{~N}$, whose emission is overlaid in red. 

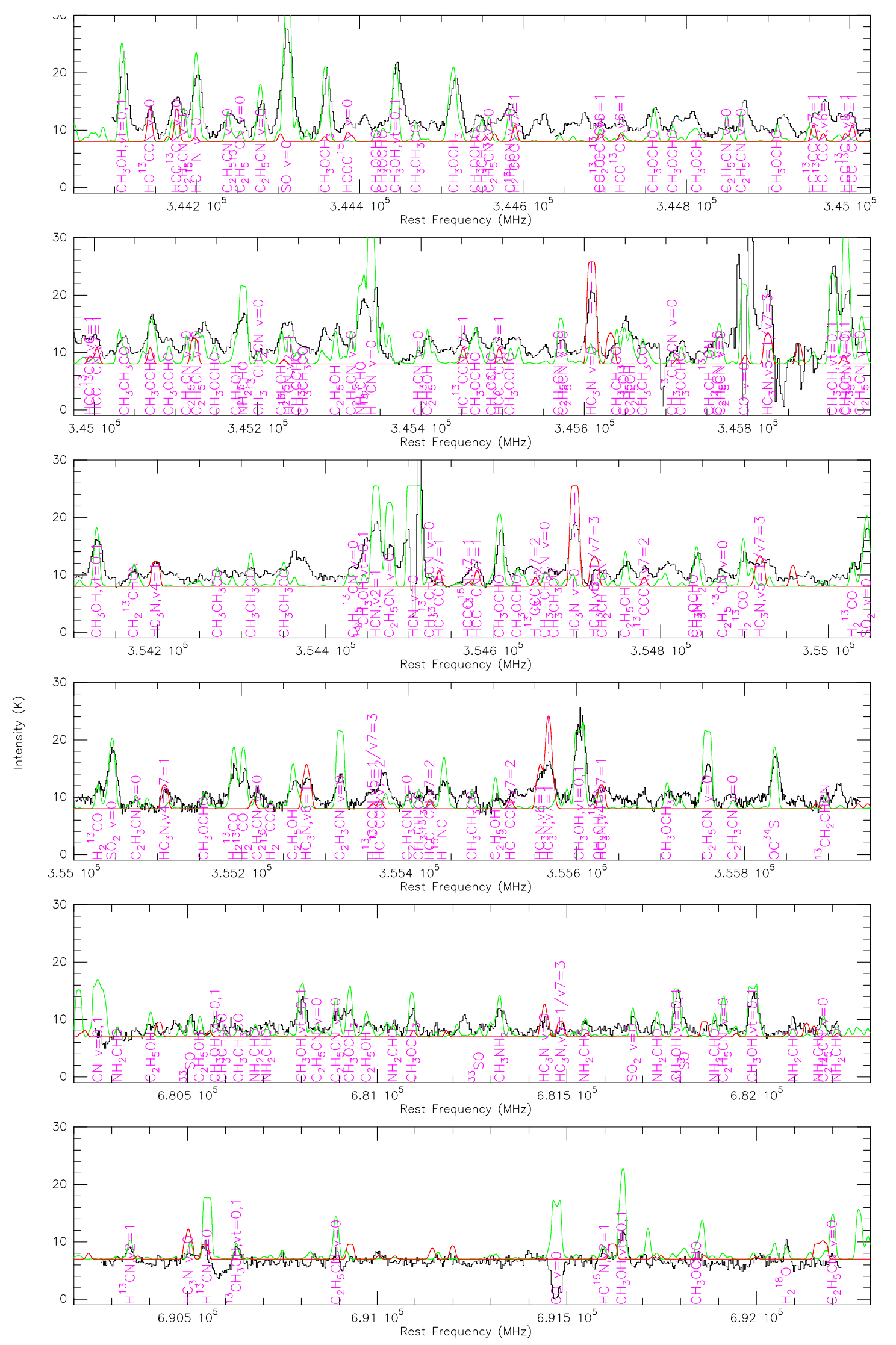

Figure 6.3: $345 / 355$ and $681 / 691 \mathrm{GHz}$ spectral range convolved to 5 " resolution. The green overlay is the model for all molecules except $\mathrm{HC}_{3} \mathrm{~N}$, whose emission is overlaid in red. 
Table 6.3: List of identified molecules

\begin{tabular}{lcc}
\hline \hline Molecule & no. lines & $\begin{array}{c}{ }^{a} \\
\text { column density } \\
\left(\mathrm{cm}^{-2}\right)\end{array}$ \\
\hline $\mathrm{SO}$ & 5 & $4(17)$ \\
$\mathrm{SO}_{2}$ & 5 & $3(17)$ \\
$\mathrm{OCS}$ & 2 & $3(18)$ \\
\hline $\mathrm{CN}$ & 1 & $\ldots{ }^{c}$ \\
$\mathrm{HCN}$ & 8 & $1(18) ; 3(18)^{d}$ \\
$\mathrm{HNC}$ & 1 & $3(17)$ \\
$\mathrm{CH}_{3} \mathrm{NH}_{2}$ & 2 & $4(17)$ \\
$\mathrm{CH}_{3} \mathrm{NC}$ & 4 & $7(15)$ \\
$\mathrm{NH}_{2} \mathrm{CN}$ & 7 & $2(16)$ \\
$\mathrm{NH}_{2} \mathrm{CHO}$ & 15 & $2(17)$ \\
$\mathrm{HC}_{3} \mathrm{~N}$ & 76 & $5(16) ; 1(18)^{d}$ \\
$\mathrm{C}_{2} \mathrm{H}_{3} \mathrm{CN}$ & 33 & $7(17)$ \\
$\mathrm{C}_{2} \mathrm{H}_{5} \mathrm{CN}$ & 55 & $9(17)$ \\
\hline $\mathrm{H}_{2} \mathrm{O}$ & 1 & $1(20)$ \\
$\mathrm{CO}$ & 2 & $\ldots$ \\
$\mathrm{H}_{2} \mathrm{CO}$ & 6 & $3(18)$ \\
$\mathrm{CH}_{3} \mathrm{OH}$ & 23 & $9(18)$ \\
$\mathrm{H}_{2} \mathrm{C}_{2} \mathrm{O}$ & 4 & $4(17)$ \\
$\mathrm{C}_{2} \mathrm{H}_{5} \mathrm{OH}$ & 23 & $6(17)$ \\
$\mathrm{CH}_{3} \mathrm{OCH}$ & 14 & $1.5(18)$ \\
$\mathrm{CH}_{3} \mathrm{OCHO}$ & 38 & $7(17)$ \\
$\mathrm{CH}_{3} \mathrm{CH} \mathrm{CH}_{3} \mathrm{CO}$ & 26 & $5(17)$ \\
\hline & &
\end{tabular}

Notes. The molecules are ordered by S-, N-, and O-bearing and by complexity. ${ }^{(a)}$ Number of identified lines (labeled in Figs. 6.2 and 6.3) ${ }^{(b)}$ Column density used in the myXCLASS model, with a source size of $1.5^{\prime \prime}$ and a temperature of $200 \mathrm{~K}$. The parentheses are powers of $10 .{ }^{(c)}$ The lines are in absorption, which is not modeled here. ${ }^{(d)}$ For $\mathrm{HCN}$ and $\mathrm{HC}_{3} \mathrm{~N}$, an additional component of $0.5^{\prime \prime}$ and $500 \mathrm{~K}$ was used to more closely match the vibrational lines.

$\mathrm{km} \mathrm{s}^{-1}$ were fixed, while the molecular column density was varied to obtain a good fit to the data (see Table 6.3). The continuum was neglected in the radiative transfer, only a foreground absorbing column density of $5 \times 10^{24} \mathrm{H}_{2}$ $\mathrm{cm}^{-2}$ was taken into account. Continuum levels of 6,8 , and $7 \mathrm{~K}$ were added to the spectra at 201/211, 345/355, and $681 / 691 \mathrm{GHz}$, respectively. Figures 6.2 and 6.3 show the spectra and the model, and Table 6.3 gives the derived column densities. We note that the model is not supposed to give an optimum fit to the data, but is just for identification purposes.

\subsubsection{Line maps}

From many maps of interesting lines (see appendix, Figs. 6.18, 6.19, and 6.20), we show here only a selection, which are relevant for the expansion motion (Fig. 6.4) and the high excitation (Fig. 6.5). They are ordered as in Table 6.3. The velocity was integrated over the ranges with detectable signal and no significant deviations in the channel maps - i.e. the whole flux for simple line shapes and several velocity ranges for more complex line shapes. For Fig. 6.4, we chose common velocity ranges for the four lines, which are $30-50 \mathrm{~km} \mathrm{~s}^{-1}$, representing the highvelocity part of the (front-side) outflow, 51-64 $\mathrm{km} \mathrm{s}^{-1}$, the low-velocity part, $65-70 \mathrm{~km} \mathrm{~s}^{-1}$, the systemic velocity, and $71-84 \mathrm{~km} \mathrm{~s}^{-1}$, the low-velocity part of the back-side outflow. Higher velocities are not detected, probably due to dust absorption. In case of contamination by a neighboring line, the velocity range was chosen to avoid this line. Still, blending is likely for $\mathrm{SO}$ at $344.3 \mathrm{GHz}$ by methanol (frequency corresponds to a $1.5 \mathrm{~km} \mathrm{~s}^{-1}$ lower velocity), $\mathrm{H}^{13} \mathrm{CN}$ at $345.3 \mathrm{GHz}$ by $\mathrm{SO}_{2}\left(1 \mathrm{~km} \mathrm{~s}^{-1}\right.$ higher velocity), $\mathrm{H}^{15} \mathrm{NC}$ at $355.4 \mathrm{GHz}$ by $\mathrm{CH}_{3} \mathrm{CH}_{3} \mathrm{CO}\left(3.3 \mathrm{~km} \mathrm{~s}^{-1}\right.$ lower velocity), and $\mathrm{CH}_{3} \mathrm{NH}_{2}$ at $354.8 \mathrm{GHz}$ by $\mathrm{CH}_{3} \mathrm{OCHO}\left(3.8 \mathrm{~km} \mathrm{~s}^{-1}\right.$ higher velocity).

\subsubsection{Spectra}

Figure 6.6 shows central spectra of the 12 transitions with absorption features. Most of the absorption is blueshifted relative to the systemic velocity of $68 \mathrm{~km} \mathrm{~s}^{-1}$. 


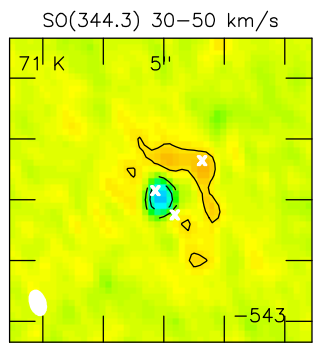

$\operatorname{HCN}(354.5) 30-50 \mathrm{~km} / \mathrm{s}$

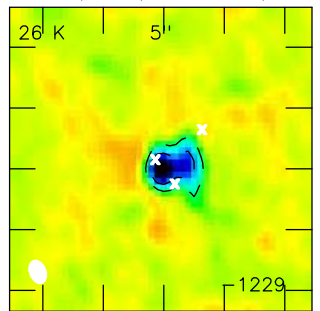

$\mathrm{HC}_{3} \mathrm{~N}, \mathrm{v}=0$ (345.6) $30-50 \mathrm{~km} / \mathrm{s}$

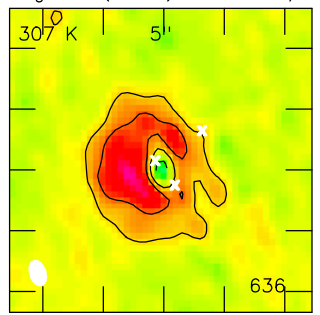

$\mathrm{CO}(345.8) 30-50 \mathrm{~km} / \mathrm{s}$

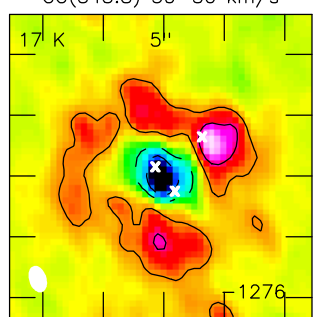

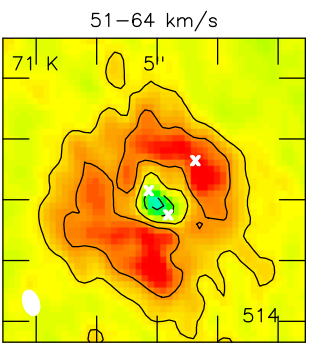

$51-64 \mathrm{~km} / \mathrm{s}$

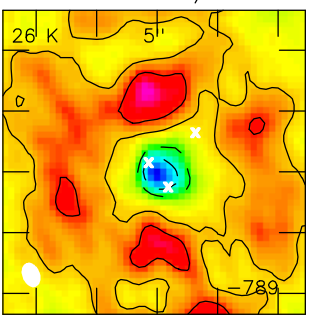

$51-64 \mathrm{~km} / \mathrm{s}$

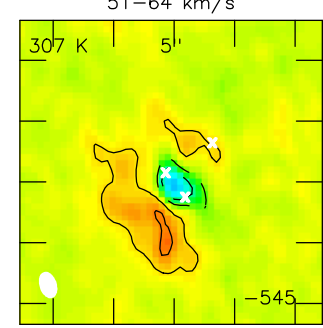

$51-64 \mathrm{~km} / \mathrm{s}$

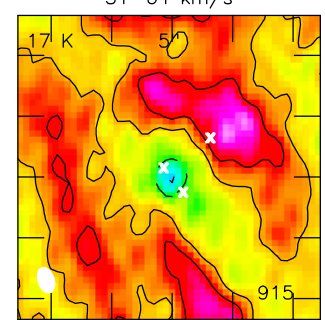

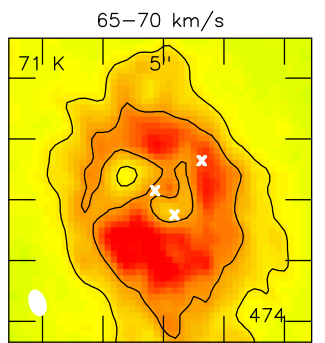

$65-70 \mathrm{~km} / \mathrm{s}$

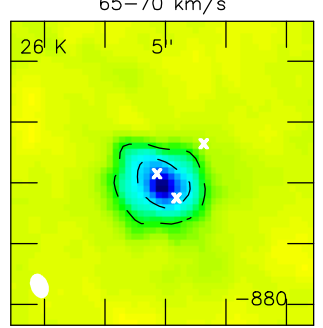

$65-70 \mathrm{~km} / \mathrm{s}$

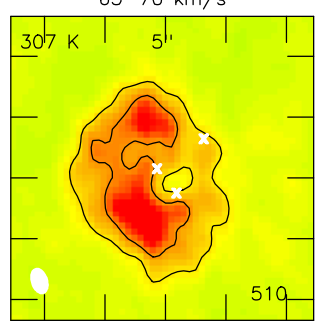

$65-70 \mathrm{~km} / \mathrm{s}$

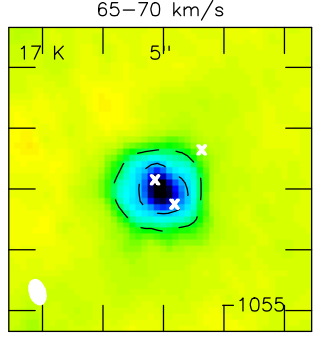

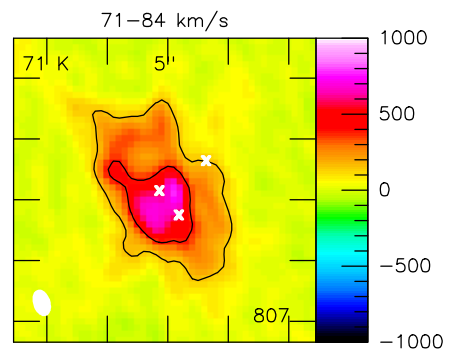

$71-84 \mathrm{~km} / \mathrm{s}$

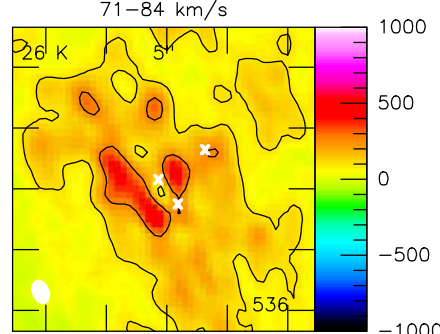

$71-84 \mathrm{~km} / \mathrm{s}$

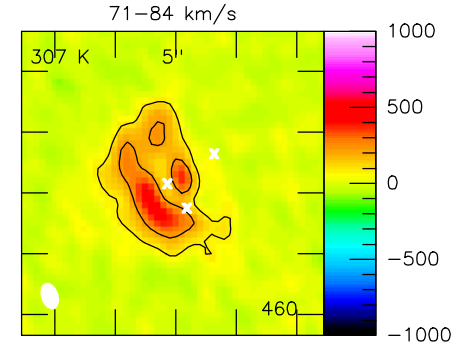

$71-84 \mathrm{~km} / \mathrm{s}$

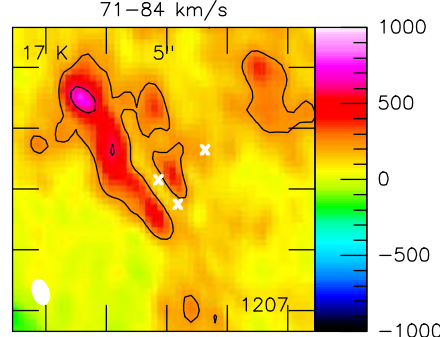

Figure 6.4: Line maps (selected to trace expansion motions) integrated over the velocity range indicated in each panel (which can be considered, from left to right, as high-velocity part of the outflow, low-velocity part, systemic velocity, and low-velocity part of the outflow at the far side). The first velocity range of $\mathrm{HC}_{3} \mathrm{~N}$ is blended by other lines, in the second velocity range of $\mathrm{CO}$ the emission is so extended to cause imaging artifacts. The map size is $5^{\prime \prime}$ (tick spaces are 1", centered on R.A. 18:08:38.236, Dec. -19:51:50.25). Beams are shown in the lower left, and the number in the lower right of each panel is the maximum flux in $\mathrm{K} \mathrm{km} \mathrm{s}^{-1}$ (contours are \pm 20 and $50 \%$ of that value). The color scale is from -1000 to $1000 \mathrm{~K} \mathrm{~km} \mathrm{~s}^{-1}$. The energy of the lower level is given in the upper left.

\subsubsection{Comparison to APEX data}

To estimate calibration and filtering of extended emission, we compare the SMA data to data from the APEX (Atacama Pathfinder Experiment) 12-m telescope (Güsten et al. 2006; Schuller et al. 2009; Rolffs et al. 2011b). The $345 \mathrm{GHz}$ flux from the LABOCA bolometer array is $34 \mathrm{Jy} / \mathrm{Beam}$, the $850 \mathrm{GHz}$ flux from SABOCA is 450 $\mathrm{Jy} / \mathrm{Beam}$. At both frequencies, the source is unresolved with the beams of 18.2 and 7.4", respectively. The spectral index is 2.86 , so the $690 \mathrm{GHz}$ flux would be $250 \mathrm{Jy}$. The SMA fluxes at 210 and $345 \mathrm{GHz}$ fit very well to the APEX data, but at $690 \mathrm{GHz}$ the continuum is much lower. This might be partly explained by decorrelation due to fast phase fluctuations, and partly by missing short baselines filtering out extended emission.

Figure 6.7 shows APEX spectra overlaid with SMA spectra which were convolved to the APEX beam (18.2" at $345 \mathrm{GHz}, 17.7^{\prime \prime}$ at $355 \mathrm{GHz}$, and 9.1" at $690 \mathrm{GHz}$ ). The lines match very well. The most notable exception is CO 6-5, which is purely in absorption with the SMA, but has strong emission as seen with APEX (>1000 Jy). Extended emission of CO 6-5 is filtered out due to missing short spacings, leaving an appearant absorption towards the continuum, which is more compact and hence not affected as much as $\mathrm{CO}$ by the filtering. 


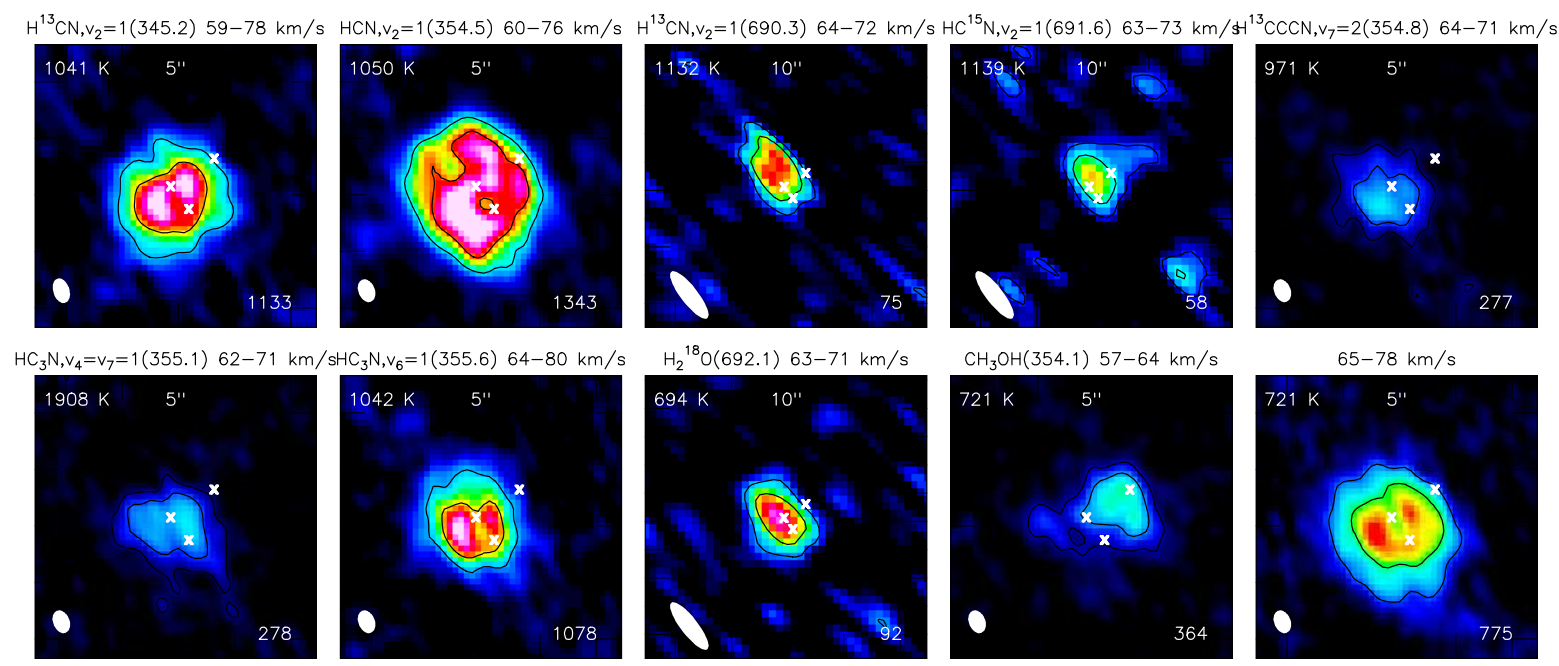

Figure 6.5: Selected high-excitation line maps. As in Fig. 6.4, but with the color scale ranging from 0 to 1000 $\mathrm{K} \mathrm{km} \mathrm{s}^{-1}$ in the $345 / 355 \mathrm{GHz}$ data and from 0 to $100 \mathrm{~K} \mathrm{~km} \mathrm{~s}^{-1}$ at $690 \mathrm{GHz}$, where the map size is $10^{\prime \prime}$.

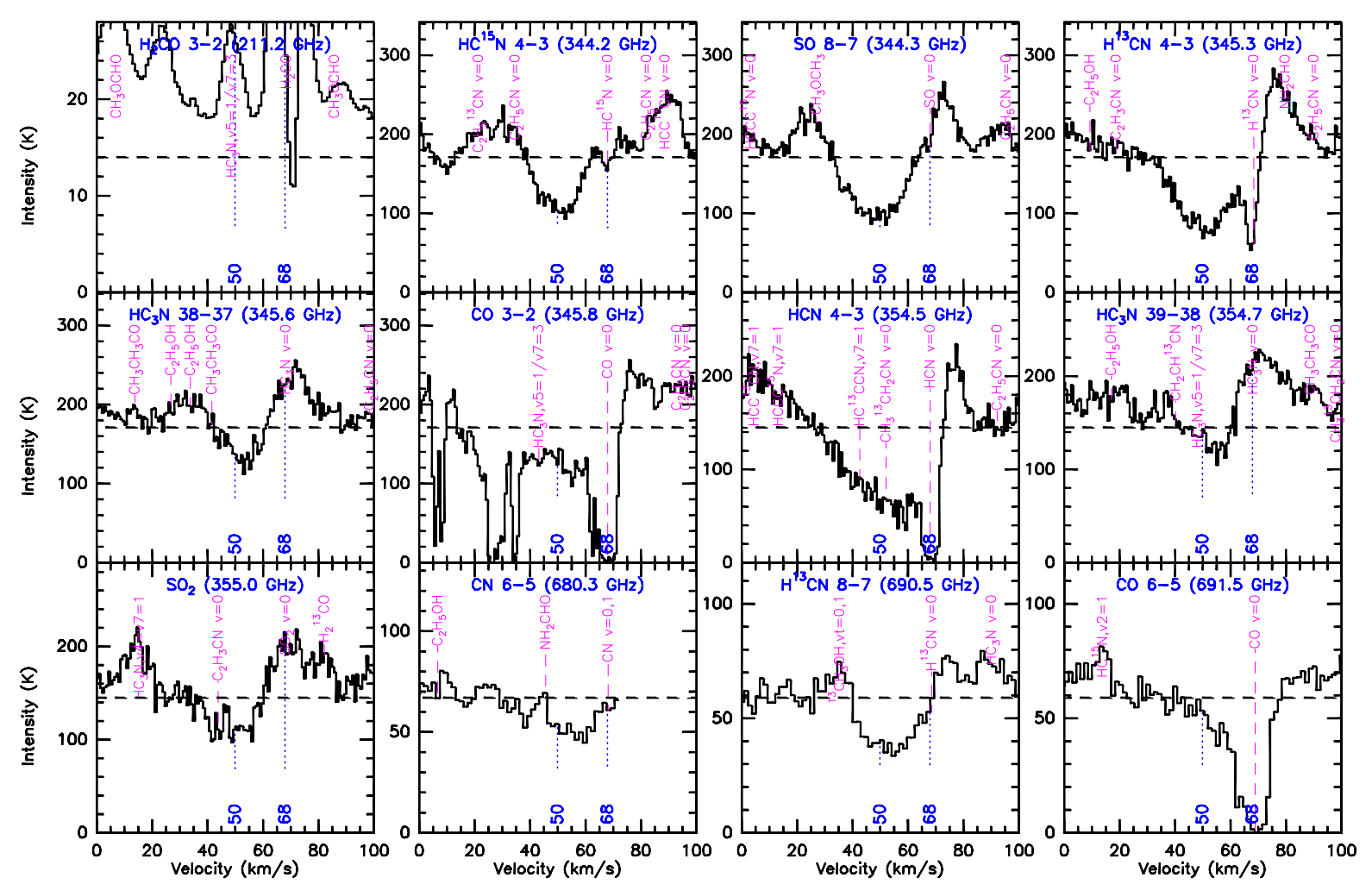

Figure 6.6: Lines with absorption features toward the continuum peak. The systemic velocity of $68 \mathrm{~km} \mathrm{~s}^{-1}$ and a blue-shifted velocity of $50 \mathrm{~km} \mathrm{~s}^{-1}$ are marked. There are absorption components centered at both velocities, indicating expansion. The dashed horizontal line denotes the continuum level. 

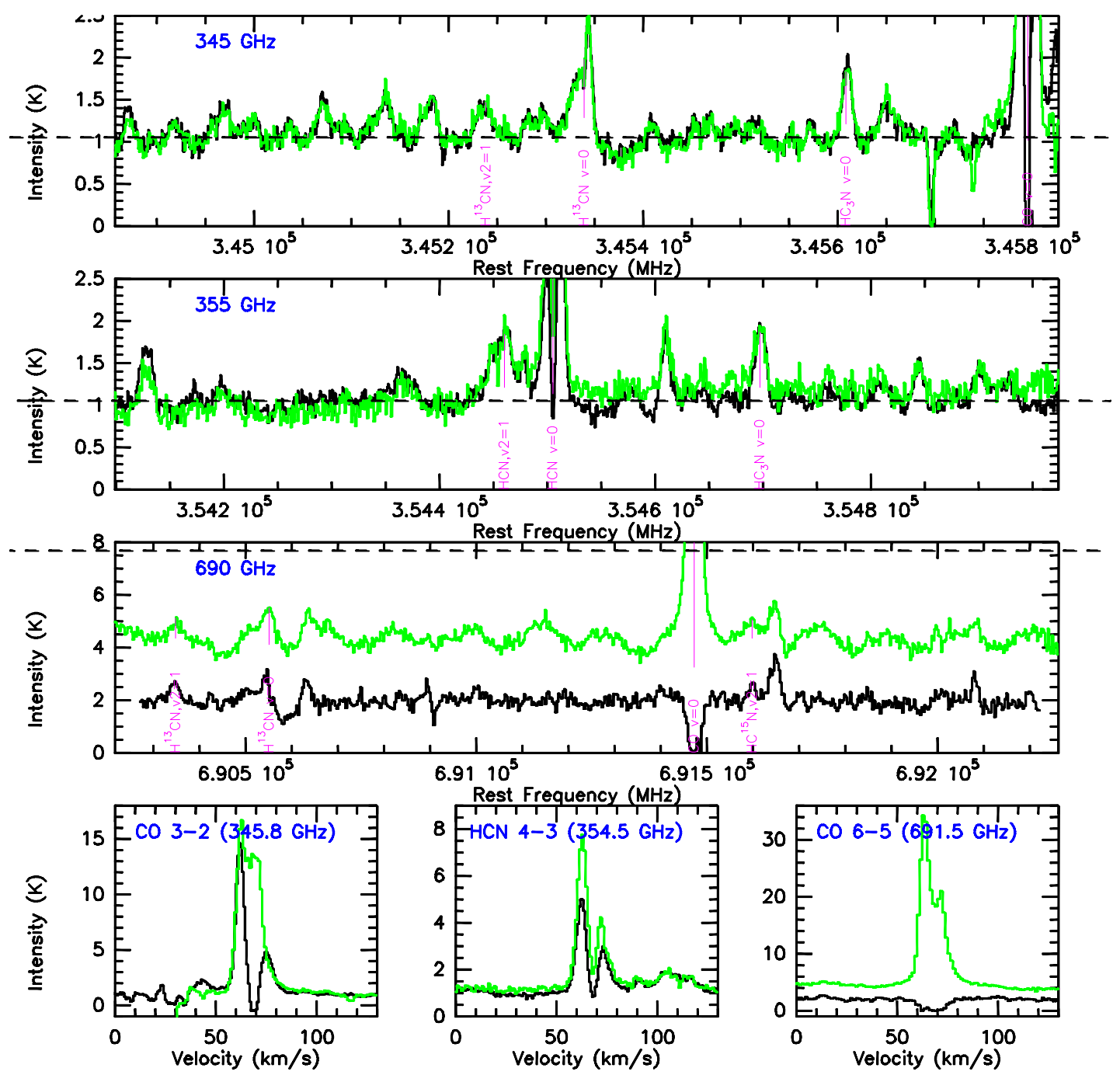

Figure 6.7: Comparison of APEX data (green) to the SMA data convolved to the APEX beam (black). The dashed lines mark the continuum level as expected from LABOCA and SABOCA measurements. The HCN and CO lines and the $690 \mathrm{GHz}$ continuum are affected by filtering of extended emission due to missing short spacings; the rest agrees very well.

\subsection{Modeling}

In this section, we compare a few models to continuum and vibrationally excited $\mathrm{HCN}$ in order to constrain the structure of this complex region, mainly density distribution and velocity field. The models are inevitably simplified, and we used a trial-and-error technique with parameter variations and comparison of model and data by eye to approach a good fit if possible for a tested structure, but no global optimization of fit parameters was performed.

The three-dimensional radiative-transfer code RADMC-3D ${ }^{8}$, developed by C. Dullemond, was employed to compute the dust temperature from stellar heating and the continuum and line emission of a model. The basic setup is the same as described in Rolffs et al. (2011a), but here we also test clumpiness and velocity structure in the models. The line transfer assumes LTE, which is a good approximation for vibrationally excited HCN, but prevents the modeling of the ground-state lines.

\subsubsection{The Models}

While no perfect fit was found, we selected five of the tested models for presentation and comparison to the data. Models A and B have been previously used to fit APEX and VLA data of this source, respectively. Model C has a radial density profile that best matches the observed continuum (if heated by the stars in the HiI regions). Models

\footnotetext{
${ }^{8}$ http://www.ita.uni-heidelberg.de/ dullemond/software/radmc-3d
} 
Table 6.4: Summary of the models

\begin{tabular}{lcccc}
\hline \hline Model & density & heating & velocity field & displayed in Figs. \\
\hline A & power-law & 1 star & infall & $6.9,6.11$ (red) \\
B & Gaussian & 3 stars & none & $6.9,6.11$ (green) \\
C & Plummer & 3 stars & none & 6.9 (blue), 6.12 (red) \\
D & clumpy & 3 stars & dispersion & 6.9 (cyan), 6.12 (green) \\
E & clumpy, outflow cone & 3 stars & dispersion, outflow & 6.9 (magenta), 6.10, 6.13-6.16 (red) \\
\hline
\end{tabular}

$\mathrm{D}$ and $\mathrm{E}$ have density fluctuations on small scales, which are expected from self-gravity and which improve the line fitting. Model $\mathrm{E}$ is an attempt to include the outflow (which is seen by the blue-shifted absorption features) and is presented in more detail.

The models are described explicitly in the following. Table 6.4 summarizes the main properties of the models.

Model A This is the model that was presented in Rolffs et al. (2011b) to fit the APEX data (continuum and many lines from $\mathrm{HCN}, \mathrm{HCO}^{+}$, and $\mathrm{CO}$, including vibrationally excited $\mathrm{HCN}$ ). The density follows a radial power law, $n=7 \times 10^{8} \times\left(\frac{r}{485 \mathrm{AU}}\right)^{-1.75} \mathrm{H}_{2} \mathrm{~cm}^{-3}$ for radii larger than $485 \mathrm{AU}$. Inside there is an HII region with an electron density of $1.5 \times 10^{6} \mathrm{~cm}^{-3}$ to reproduce the free-free radiation from $\mathrm{B} 1$, and a star of $5.6 \times 10^{5} \mathrm{~L}_{\odot}$. The dust opacity is from Ossenkopf \& Henning (1994) without grain mantles, but with coagulation at a density of $10^{5} \mathrm{~cm}^{-3}$. The $\mathrm{HCN}$ abundance is $3 \times 10^{-5}$ at temperatures above $100 \mathrm{~K}$ and $5 \times 10^{-8}$ below $100 \mathrm{~K}$. The linewidth is $5 \mathrm{~km} \mathrm{~s}^{-1}$ FWHM, and the gas is infalling with $1 \mathrm{~km} \mathrm{~s}^{-1}$. The total mass (in a cube of $3 \mathrm{pc}$ diameter) is $2.4 \times 10^{4} \mathrm{M}_{\odot}$, of which $70 \mathrm{M}_{\odot}$ are at temperatures above $300 \mathrm{~K}$.

Model B This is the model that was presented in Rolffs et al. (2011a) to fit the VLA data of vibrationally excited $\mathrm{HCN}$. The density follows a Gaussian, centered at B1, with $7 \times 10^{7} \mathrm{H}_{2} \mathrm{~cm}^{-3}$ at the half-maximum radius of 7000 AU. As in all following models, heatings sources are the stars in the HII regions B1 with $10^{5} \mathrm{~L}_{\odot}$, B2 with $8.3 \times 10^{4}$ $\mathrm{L}_{\odot}$, and A with $4.6 \times 10^{4} \mathrm{~L}_{\odot}$. The dust opacity is from Ossenkopf \& Henning (1994) without grain mantles or coagulation, as in all following models. The HCN abundance is $10^{-5}$. The intrinsic line width (FWHM) is 8.3 $\mathrm{km} \mathrm{s}^{-1}$, and there is no macroscopic velocity field. The total mass is $3.5 \times 10^{3} \mathrm{M}_{\odot}$, of which $350 \mathrm{M}_{\odot}$ are at temperatures above $300 \mathrm{~K}$.

Model C The density in this model follows a Plummer profile, $n=1.4 \times 10^{8} \times\left(1+\left(\frac{r}{11500 \mathrm{AU}}\right)^{2}\right)^{-2.5} \mathrm{H}_{2} \mathrm{~cm}^{-3}$ (half-maximum radius $6500 \mathrm{AU}$ ). It is centered $2000 \mathrm{AU}$ south and $1000 \mathrm{AU}$ west of $\mathrm{B} 1$. The Plummer model is very similar to a Gaussian inside the half-maximum radius, but has higher density outside (still falling off steeply as $r^{-5}$ ). The $\mathrm{HCN}$ abundance is $10^{-5}$ at temperatures above $300 \mathrm{~K}$ and $10^{-6}$ below, as in the following models. The intrinsic line width (FWHM) is $8.3 \mathrm{~km} \mathrm{~s}^{-1}$, and there is no macroscopic velocity field. The total mass is $6.8 \times 10^{3}$ $\mathrm{M}_{\odot}$, of which $350 \mathrm{M}_{\odot}$ are at temperatures above $300 \mathrm{~K}$.

Model D This model consists of 100 clumps, which have a Plummer half-maximum radius of $1000 \mathrm{AU}$ and central densities ranging from $4 \times 10^{8}$ to $2 \times 10^{9} \mathrm{H}_{2} \mathrm{~cm}^{-3}$ (the mass of the Gaussian clump would be five times the stellar mass, according to the Initial Mass Function). They are randomly placed in the model according to a Plummer distribution with half-maximum radius 4000 AU. The density in each model cell is the maximum of all contributions from the clumps. So with a lot of overlap, this structure consists rather of density fluctuations than of separate cores. The intrinsic line width (FWHM) is $5 \mathrm{~km} \mathrm{~s}^{-1}$. Each clump has a random line-of-sight velocity with Gaussian half-maximum value of $\pm 5 \mathrm{~km} \mathrm{~s}^{-1}$. The line-of-sight velocity of each cell is an average of all clumps, weighted with their density contribution. The total mass is $9.4 \times 10^{3} \mathrm{M}_{\odot}$, of which $470 \mathrm{M}_{\odot}$ are at temperatures above $300 \mathrm{~K}$.

Model E This model has the same structure as model D, but a half-maximum radius of the distribution of the clumps of only $3000 \mathrm{AU}$. In addition, a bipolar outflow is present, with an opening angle of $100^{\circ}$ and a length of $2 \times 10^{4} \mathrm{AU}$. Its center lies $1000 \mathrm{AU}$ south and $500 \mathrm{AU}$ west of B1. The axis is tilted from the line of sight by $20^{\circ}$, and the foreground part directed towards B2. Inside the outflow cone, the density is reduced to $20 \%$ and the velocity is $10 \mathrm{~km} \mathrm{~s}^{-1}$ outwards. This is only a toy model of the outflow. The total mass is $4.6 \times 10^{3} \mathrm{M}_{\odot}$, of which $400 \mathrm{M}_{\odot}$ are at temperatures above $300 \mathrm{~K}$. The clumpy structure is visualized in Fig. 6.8. 


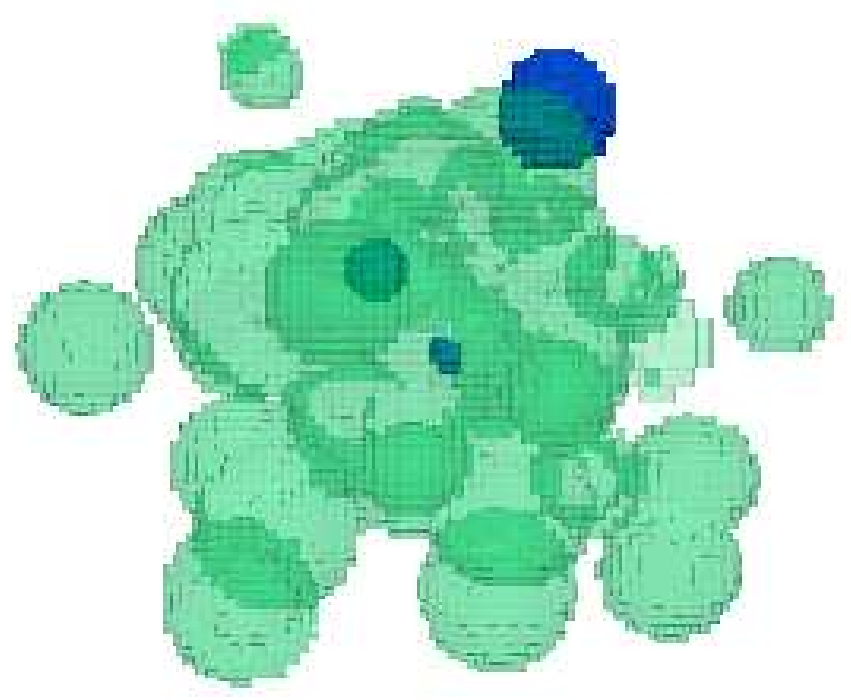

Figure 6.8: Clumpy structure of model E displayed as isocontours of $10^{7} \mathrm{H}_{2} \mathrm{~cm}^{-3}$ (green, transparent). The HII regions, whose stars heat up the gas, are shown in blue. The size of the contoured region is around $0.2 \mathrm{pc}$.

\subsubsection{Continuum}

The continuum radiation that the models emit is compared to the data. Models D and E have randomly placed core centers; therefore their radiation (continuum and lines) have a random component, which cannot be exactly reproduced in different runs with the same parameters.

The radial profile of the $345 / 355 \mathrm{GHz}$ continuum map is extracted with central position R.A. 18:08:38.237, Dec. - 19:51:50.421, which is the center of a two-dimensional Gaussian fit $\left(1.4^{\prime \prime} \times 1.17^{\prime \prime}\right.$, elongated along the B1B2 axis). The model maps were Fourier-transformed, folded with the uv-coverage of the observations, and imaged in the same way as the data. The radial profile of the model was extracted from the same central coordinates. Figure 6.9 shows a comparison of the radial profiles, Fig. 6.10 a comparison of the continuum map of model E to the data.

The flux from APEX/LABOCA (345 GHz) is $34 \mathrm{Jy} /$ Beam (18.2" beam size) and from APEX/SABOCA (850 $\mathrm{GHz}) 450 \mathrm{Jy} /$ Beam (7.4" beam size). The models emit 31 (A), 20 (B), 26 (C), 20 (D), and 18 (E) Jy/Beam for LABOCA and 266 (A), 231 (B), 311 (C), 146 (D), and 162 (E) Jy/Beam for SABOCA. In models B-E an extended component similar to A could be added without affecting the results of the interferometer modeling.

\subsubsection{Vibrationally excited HCN}

The models are compared to lines from vibrationally excited $\mathrm{HCN}$, including the $J=13$ direct $\ell$-type transition at 40.7669 GHz observed with the VLA (Rolffs et al. 2011a). Figures 6.11 and 6.12 show this line and three rotational transitions of vibrationally excited HCN observed with the SMA, overlaid with models A-D. Model E is shown in more detail in comparison to the $J=13$ direct $\ell$-type line of vibrationally excited HCN (Fig. 6.13), the 4-3 line of vibrationally excited $\mathrm{HCN}$ (Fig. 6.14) and $\mathrm{H}^{13} \mathrm{CN}$ (Fig. 6.15), and the 8-7 transition of vibrationally excited $\mathrm{H}^{13} \mathrm{CN}$ (Fig. 6.16). The levels of all these transitions lie between 1050 and $1400 \mathrm{~K}$ above ground.

\subsection{Discussion}

\subsubsection{Chemistry}

In addition to about 350 identified lines, there are around 90 unidentified (U-) lines. 75 of these 90 U-lines are in the $201 / 211 \mathrm{GHz}$ range, which covers a similar velocity range as the higher-frequency bands combined (almost $6000 \mathrm{~km} \mathrm{~s}^{-1}$ ). This is probably due to the lower noise at these frequencies. The line density is mainly determined by the width of single lines $\left(5-10 \mathrm{~km} \mathrm{~s}^{-1}\right)$, i.e. a forest of overlapping lines covers most of the bands. The identified molecules (3 S-bearing, $10 \mathrm{~N}$-bearing, and 9 other, O-bearing, molecules) represent only the strongest lines, while more complex molecules have many weak lines which probably add up to the continuum and are undetectable due 


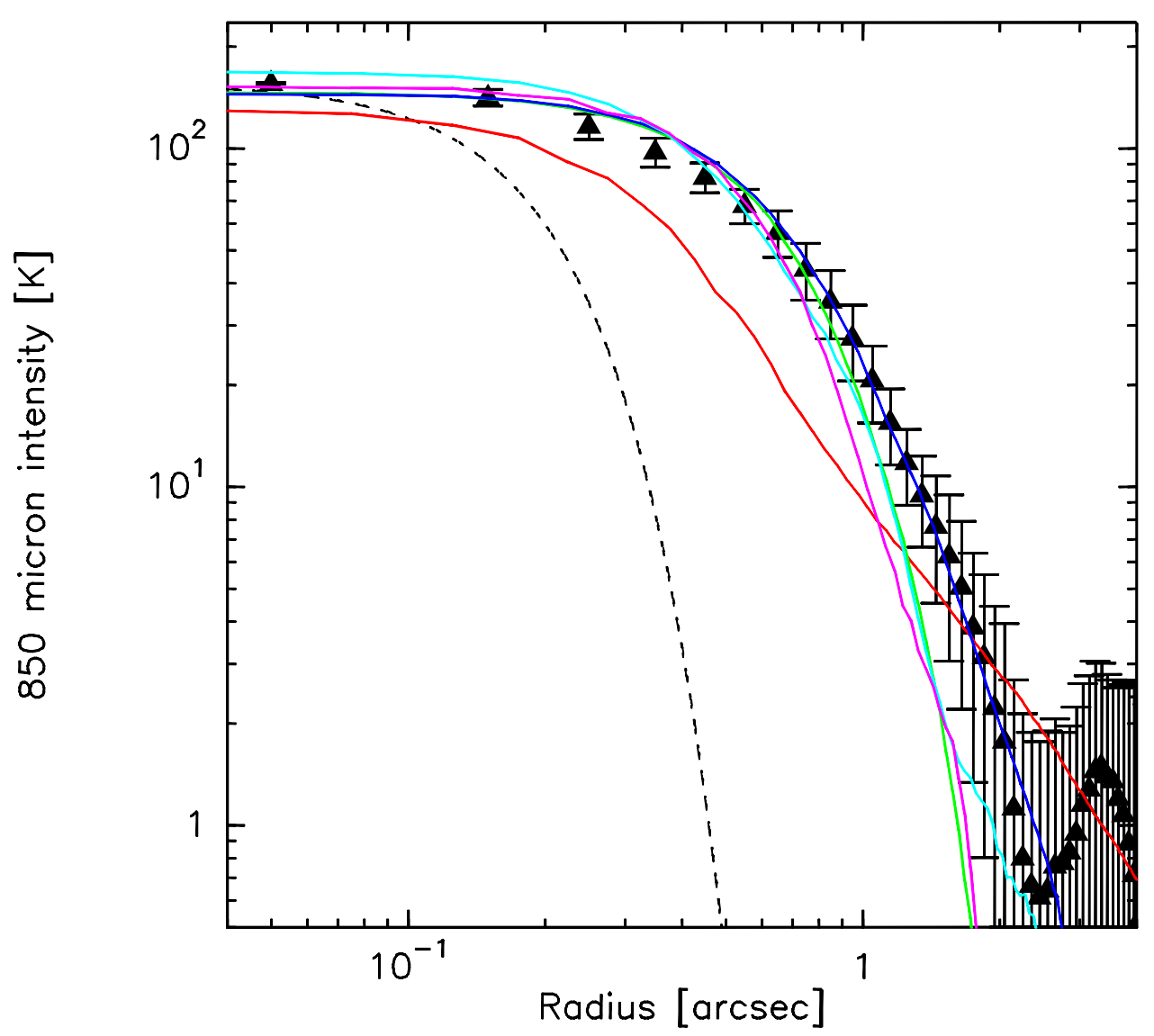

Figure 6.9: Models A (red), B (green), C (blue), D (cyan), and E (magenta) compared to the SMA continuum profile, which is displayed as data points with a $0.1^{\prime \prime}$ radial interval. The errorbars denote the rms deviation from a circular shape, and the dashed curve represents the beam.

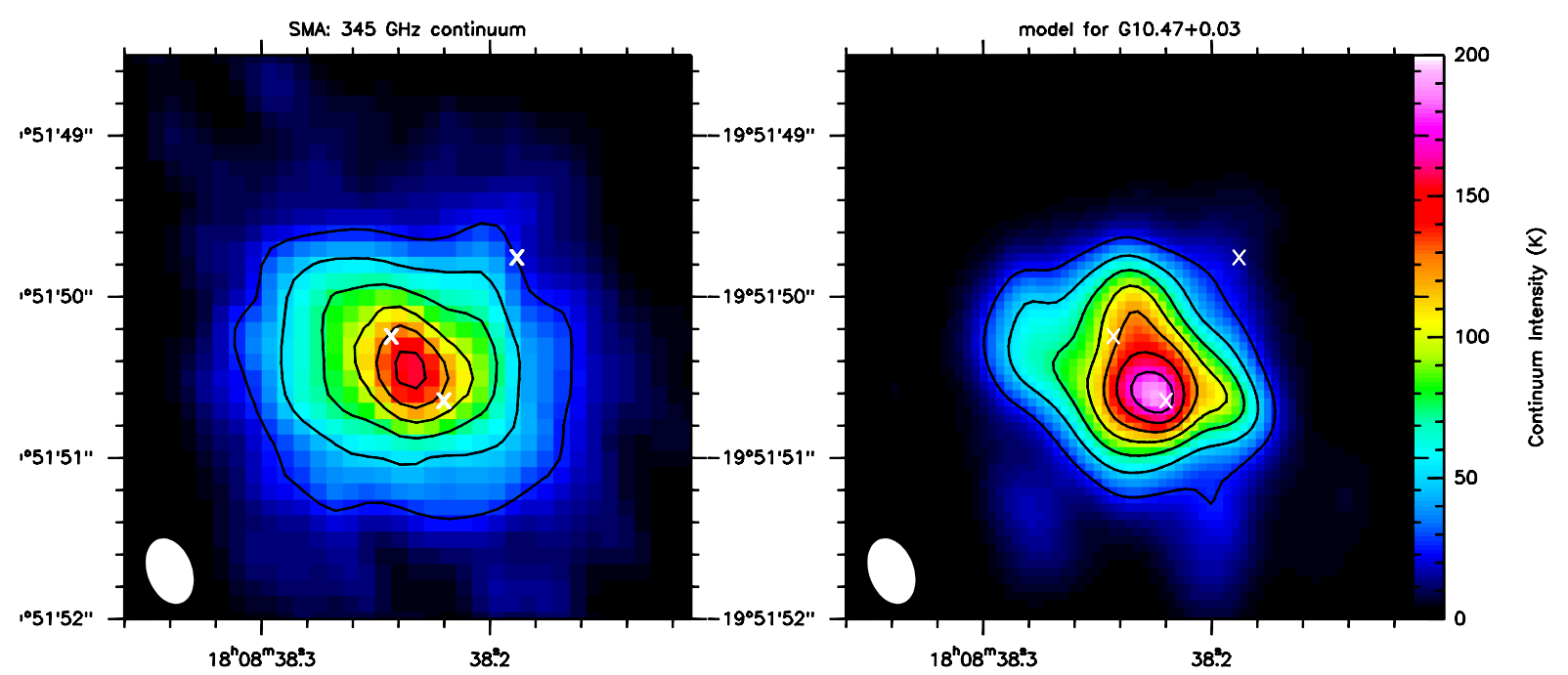

Figure 6.10: Model E (right) compared to the SMA continuum map at $345 \mathrm{GHz}$ (left). 

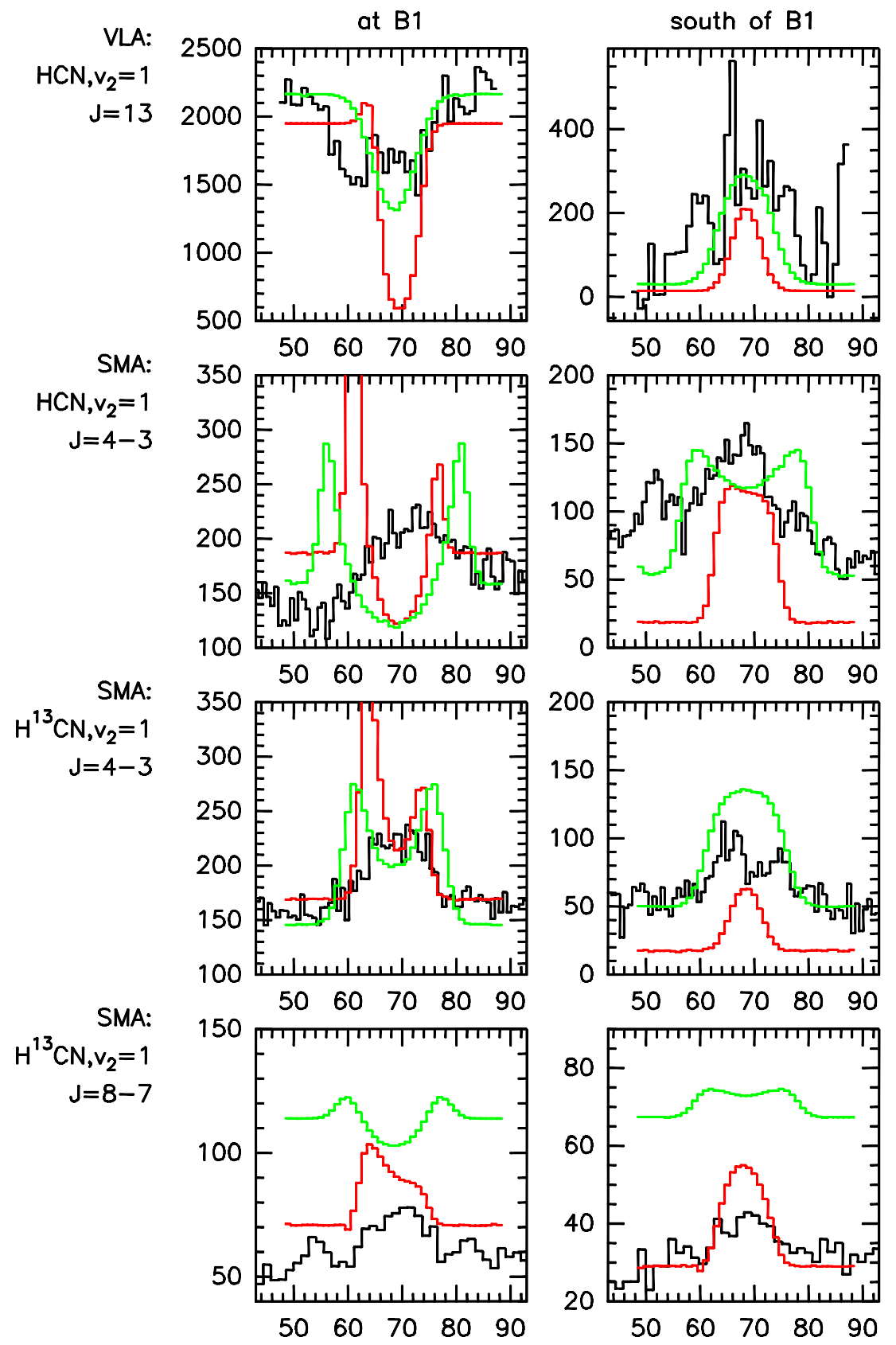

Figure 6.11: Models A (red) and B (green) compared to lines from vibrationally excited $\mathrm{HCN}$ at the position of $\mathrm{B} 1$ and $0.3^{\prime \prime}$ (VLA) or $0.8^{\prime \prime}$ (SMA) south of B1.

to line confusion. No spatial separation of different molecules could be seen. The differences in the maps can probably be explained by excitation and optical depth.

As the detailed models show, the abundance of $\mathrm{HCN}$ in the hot gas is very high, on the order of $10^{-5}$. The abundance of $\mathrm{HC}_{3} \mathrm{~N}$ seems to follow a similar increase at high temperatures. Notable line detections are $\mathrm{HC}^{15} \mathrm{~N}, v_{2}=1$ at $691.6 \mathrm{GHz}$ (lower level is $1139 \mathrm{~K}$ above ground), $\mathrm{HC}_{3} \mathrm{~N}, v_{4}=v_{7}=1$ at $355.1 \mathrm{GHz}(1908 \mathrm{~K})$, and the ${ }^{13} \mathrm{C}$ substituted $\mathrm{HC}_{3} \mathrm{~N}, v_{7}=2(970 \mathrm{~K})$. The $\mathrm{H}_{2}^{18} \mathrm{O}$ line at $692.1 \mathrm{GHz}(694 \mathrm{~K})$ implies a high abundance of water in the dense, warm gas. These abundance enhancements could be connected, e.g. through a reduced abundance of $\mathrm{OH}$, which at high temperatures reacts to form water instead of destroying N-bearing molecules (Rodgers \& Charnley 2001).

\subsubsection{Density Distribution}

As the high-resolution continuum map at $345 / 355 \mathrm{GHz}$ shows, a power-law radial density is not consistent with the data. A Gaussian fits well in the inner part, but falls off too steeply in the outer parts. What fits best to the data is a Plummer model (Fig. 6.9, blue). This profile is very similar to a Gaussian inside the half-maximum radius, but is 

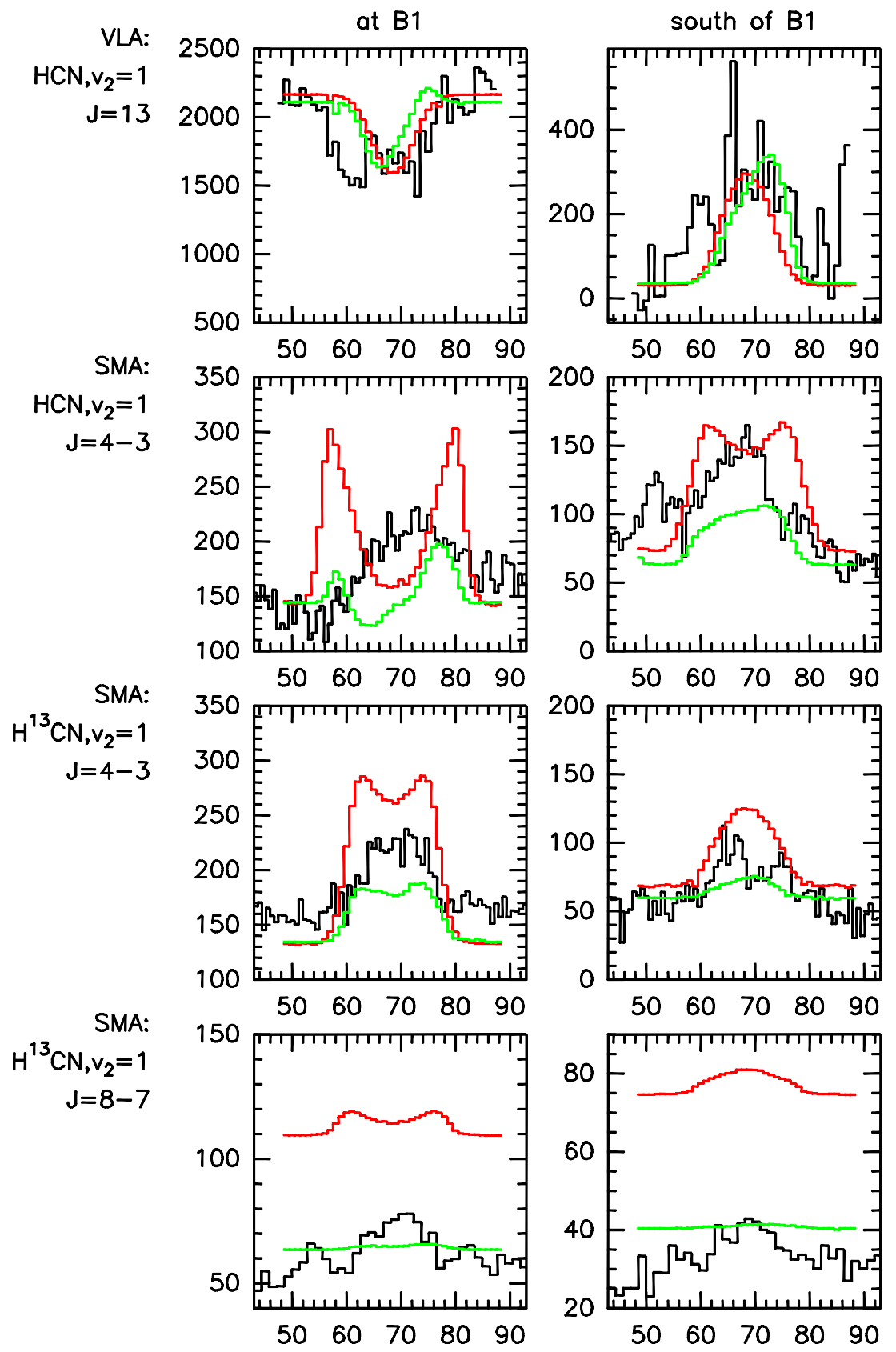

Figure 6.12: Models C (red) and D (green) compared to lines from vibrationally excited HCN at the position of B1 and $0.3^{\prime \prime}$ (VLA) or $0.8^{\prime \prime}$ (SMA) south of B1.

denser outside, where it falls off as $r^{-5}$. It is also used to describe the stellar density of star clusters. Although that might reveal a connection of this forming star cluster to later stages, it could also have completely different physical reasons. The stellar density distribution is determined by stellar dynamics, while the gas density distribution is determined by the interplay of different sources of pressure (gravitational versus turbulent, rotational, magnetic, radiative, and thermal pressure). The observed central flattening of the density can be explained by centrally increased pressure, which is expected from feedback by the newly formed massive stars. This pressure stalls the infall and piles up the mass.

The SMA continuum at $690 \mathrm{GHz}$ is rather weak compared to the $345 \mathrm{GHz}$ flux and the high-frequency singledish flux. As interferometer observations at these frequencies are challenging and only very few have been conducted yet (e.g. Beuther et al. 2006), this could be due to decorrelation caused by rapid phase noise. On the other hand, the line strengths are similar to the APEX data (Fig. 6.7), so if the low continuum is real, it can be due to optical depth effects in very dense, small condensations, while extended emission is filtered out. The continuum at $690 \mathrm{GHz}$ must be more extended than at $345 \mathrm{GHz}$ because the larger dust opacity makes it stronger only in the optically thin regions, while it can be weaker in the optically thick case due to the colder foreground. In addition, 


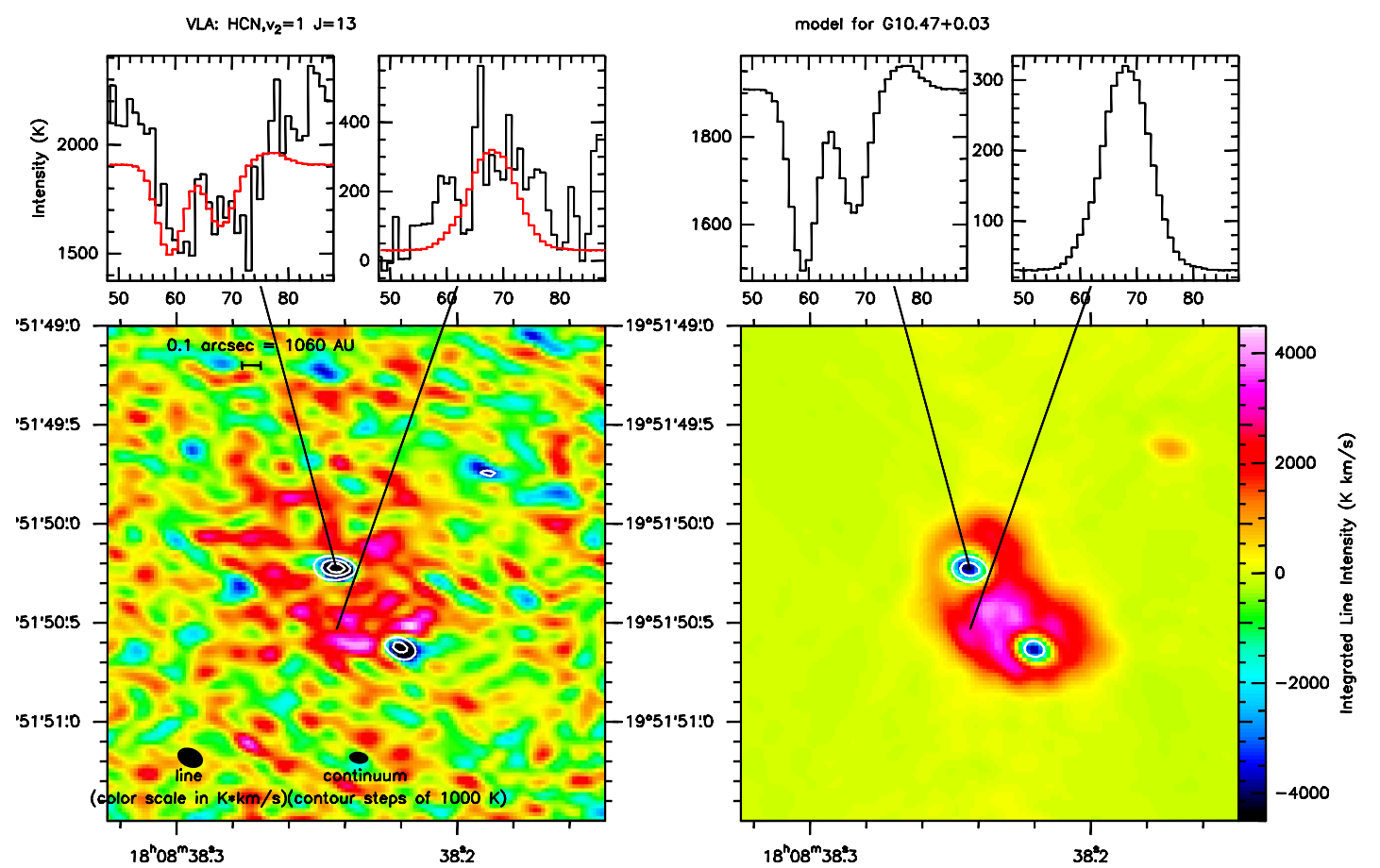

Figure 6.13: Model E (right) compared to the $J=13$ direct $\ell$-type line of vibrationally excited $\mathrm{HCN}$ at $40.8 \mathrm{GHz}$ (VLA), shown as integrated line map and spectra at two locations (left, model spectra are overlaid in red). The white contours denote the $7 \mathrm{~mm}$ continuum in steps of $1000 \mathrm{~K}$.

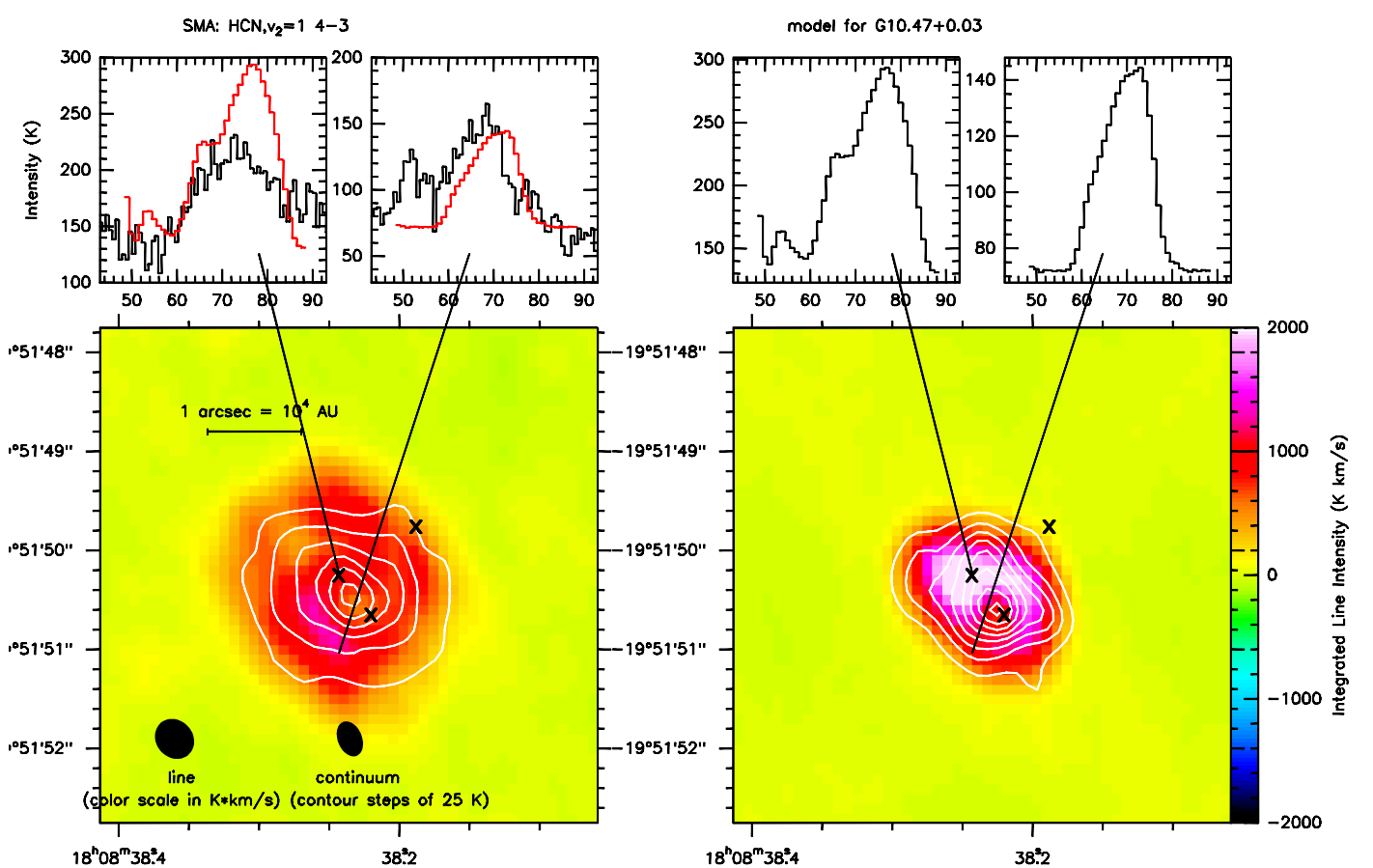

Figure 6.14: Model E (right) compared to the $J=4-3$ line of vibrationally excited $\mathrm{HCN}$ at $354.5 \mathrm{GHz}$ (SMA), shown as integrated line map and spectra at two locations (left, model spectra are overlaid in red). The white contours denote the $355 \mathrm{GHz}$ continuum in steps of $25 \mathrm{~K}$. 


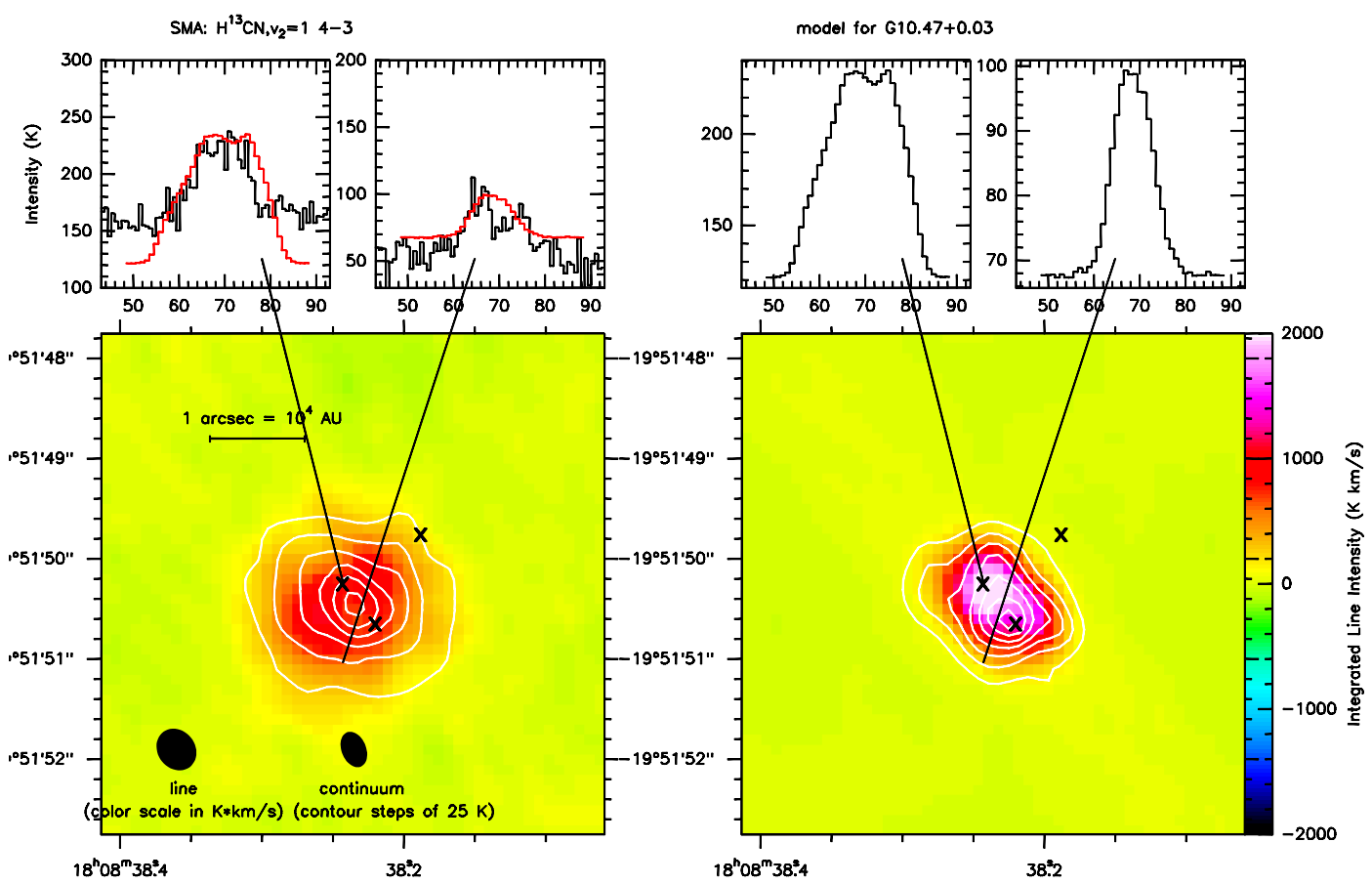

Figure 6.15: Model E (right) compared to the $J=4-3$ line of vibrationally excited $\mathrm{H}^{13} \mathrm{CN}$ at $345.2 \mathrm{GHz}$ (SMA), shown as integrated line map and spectra at two locations (left, model spectra are overlaid in red). The white contours denote the $345 \mathrm{GHz}$ continuum in steps of $25 \mathrm{~K}$.

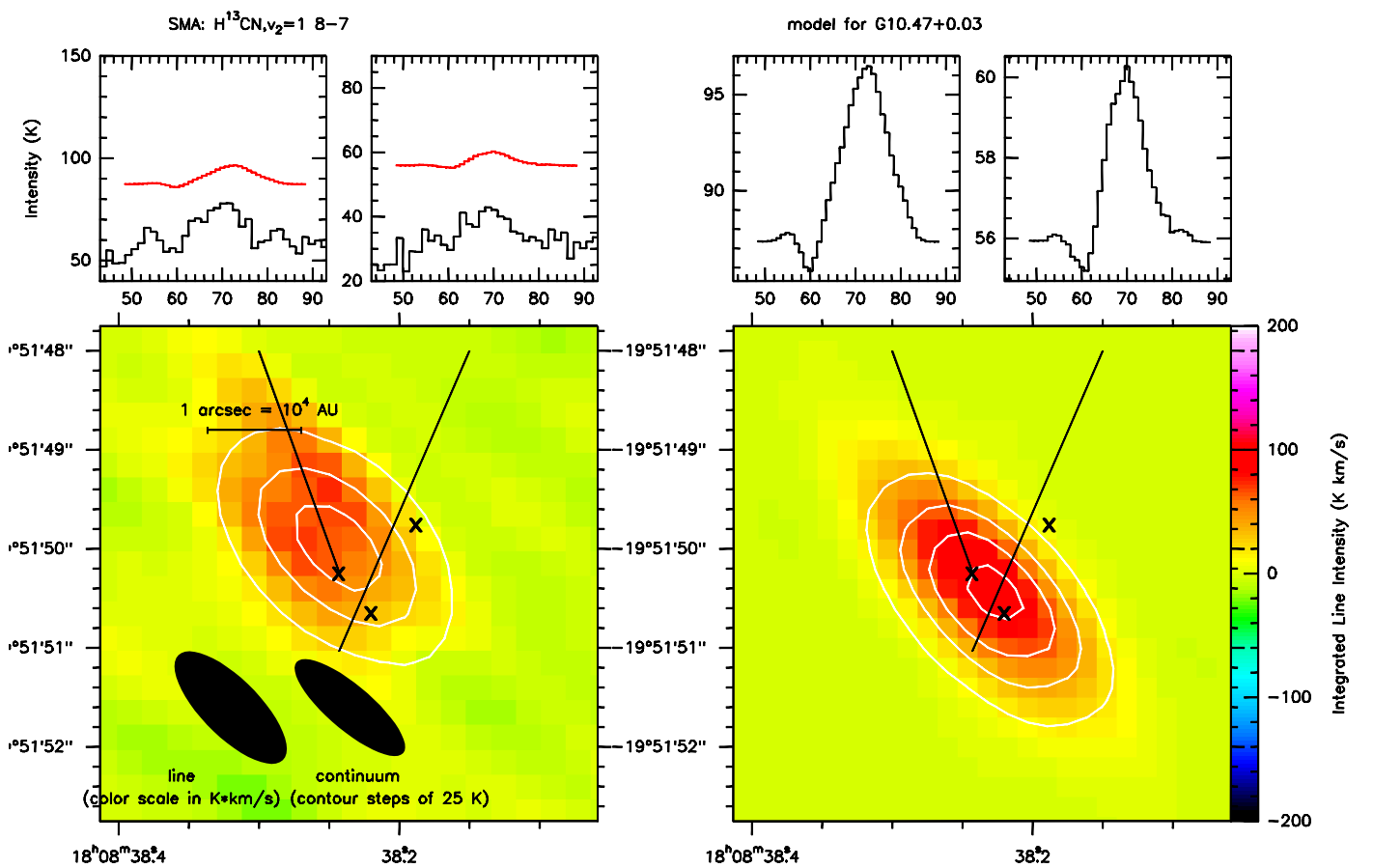

Figure 6.16: Model E (right) compared to the $J=8-7$ line of vibrationally excited $\mathrm{H}^{13} \mathrm{CN}$ at $690.4 \mathrm{GHz}$ (SMA), shown as integrated line map and spectra at two locations (left, model spectra are overlaid in red). The white contours denote the $690 \mathrm{GHz}$ continuum in steps of $25 \mathrm{~K}$. 
the minimum baselines at $690 \mathrm{GHz}$ are much longer than at $345 \mathrm{GHz}$.

There is no fragmentation observed in neither the continuum nor the lines. The reason may be that we do not resolve structures smaller than the beam size of about $3000 \mathrm{AU}$. We note that the continuum source extends over about 10 beams ( $\left.3^{\prime \prime}\right)$, so no large-scale fragmentation is present. Models of the line emission reveal that a spherical density distribution leads to strong self-absorption features, which are not observed. Clumpiness better reproduces the line shapes, but without sufficient spatial resolution we cannot derive the properties of fragmentation.

This missing fragmentation is similar to the contiuum data recently obtained by Qin et al. (2011) for SgrB2-N, which follows a very similar radial profile and also displays a similar spectral line content. Maybe the two sources are at the same evolutionary stage, where the first hypercompact HII regions have developed and heated the gas, but large-scale fragmentation has not set in.

The rotational transition of vibrationally excited $\mathrm{HCN}$ (4-3 at $354.46 \mathrm{GHz}$ ) has a very high optical depth. The Einstein A coefficient is 50,000 times larger than for the direct $\ell$-type line $(J=13$ at $40.7669 \mathrm{GHz}$, observed with the VLA), which has an optical depth of $\lesssim 1$. It must be self-absorbed, as also the comparison to the $\mathrm{H}^{13} \mathrm{CN}$ line shows (Fig. 6.5), but this is not obvious in the line shape. The single-peaked profile can only be explained by an inhomogeneous density and velocity field, since the temperature gradient otherwise leads to a strong double-peaked profile.

The observed continuum depends on density, temperature, and dust opacity. The density can vary by many orders of magnitude, and therefore dominates the resulting continuum. It is clear that the temperature is high in the inner region and falls off outwards; this is included in the models as the stars in the hypercompact HII regions heat the dust. It is entirely possible, however, that more heating sources are present. The extension of high-excitation lines is generally a bit smaller in the models than in the data, especially highly excited $\mathrm{HC}_{3} \mathrm{~N}$ is somewhat extended to the east (Fig. 6.5).

\subsubsection{Velocity Field}

While most lines do not show any velocity structure in their maps, there seems to be a component of highly excited $\mathrm{CH}_{3} \mathrm{OH}$ at lower velocity close to the UCHII region A (Fig. 6.5). Alternatively, this could arise from blending by a different line.

On large scales, the asymmetries of self-absorbed lines clearly indicate infall motions (Rolffs et al. 2011b, and Fig. 6.7), and also the absorption of the $\mathrm{H}_{2} \mathrm{CO}$ line is a bit red-shifted (Fig. 6.6). On smaller scales, we see expansion motions, as very nicely traced by the absorption features (Fig. 6.6). The blue-shifted absorption is stronger in the western part of the continuum. In addition, the SO line at $344.3 \mathrm{GHz}$, the $\mathrm{HCN}$ line at $354.5 \mathrm{GHz}$, and the $\mathrm{CO}$ line at $345.8 \mathrm{GHz}$ have red-shifted emission in the north-eastern part. A red-shifted clump 5" north of the hot core, which is only seen in $\mathrm{HCN}$ and CO, seems to be too far away to be related to the outflow (Fig. 6.4).

Olmi et al. (1996) detected a north-south velocity gradient in ${ }^{13} \mathrm{CO}$, and Hofner \& Churchwell (1996) see blueshifted water masers $0.2^{\prime \prime}$ north and $0.4^{\prime \prime}$ south of $\mathrm{B}$, and a red-shifted water maser (at $70 \mathrm{~km} \mathrm{~s}^{-1}$ ) $1.2^{\prime \prime}$ north of B1. Absorption against the hypercompact HII regions B1 and B2 is found to be blue-shifted in $\mathrm{NH}_{3}(4,4)($ Cesaroni et al. 2010, $53 \mathrm{~km} \mathrm{~s}^{-1}$ toward B1 and $61 \mathrm{~km} \mathrm{~s}^{-1}$ toward B2), and also partly in vibrationally excited HCN (Rolffs et al. 2011a, and Fig. 6.13).

One can conclude that there is likely a bipolar outflow, whose foreground part is tilted to the south-west, and whose background part is tilted to the north-east. A sketch of the outflow scenario is presented in Fig. 6.17. This scenario explains qualitatively all observed velocity features. The different $\mathrm{NH}_{3}(4,4)$ velocities, for instance, can be understood as an effect of the projection of the outflow to the line-of-sight. An embedded source, which has not yet developed a detectable HII region, is driving the outflow in this scenario. A nearly spherical infall with lower velocity, but higher rate than the expansion surrounds the outflow and produces the blue asymmetric line profiles seen on larger scales. This accretion probably continues well into the central region along certain paths, driven by gravitational attraction and the momentum of the large-scale accretion flow.

An alternative scenario might arise if the hypercompact HII regions B1 and B2 are not spherical, but in the form of bipolar expanding bubbles from a photoevaporating disk (Hollenbach et al. 1994). In this case, the stars in the HCHII regions would drive two outflows, which are both roughly aligned with the line-of-sight.

In addition, it is possibile that the source is a large pseudo-disk accreting from a massive envelope, rotating roughly in the plane of the sky, driving an outflow perpendicular to it, and having formed B1 and B2 as binaries (and maybe more stars). This would correspond to the transition between phase II and III of Zapata et al. (2010).

A quantitative radiative transfer model, which reproduces all observed lines, is possible, but beyond the scope of this paper. That the outflow is important for reproducing the line shapes has been demonstrated in our modeling (Sect. 6.6). 


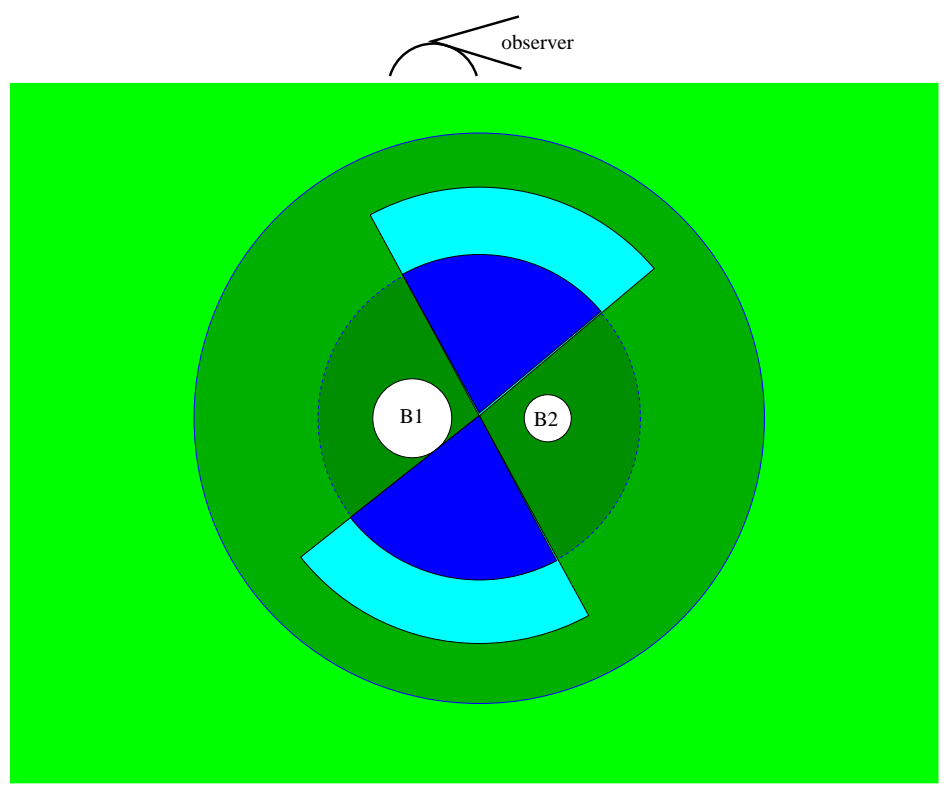

Figure 6.17: Qualitative scenario for the outflow in G10.47+0.03. The observer is above the plot. Infalling molecular gas is shown in green, expanding molecular gas in blue, and ionized gas in white. The circles denote half-maximum and Plummer radius in Model C (the gas must be clumpy, though) and the boundaries of the HII regions, which have density gradients. If the HII regions are not spherical, the outflow could also originate from the ionizing stars.

\subsection{Conclusions}

With the SMA, we have obtained high-resolution, spectrally resolved maps of the massive hot molecular core G10.47+0.03 at different frequencies, covering a bandwidth of $12 \mathrm{GHz}$ in total and including observations at $690 \mathrm{GHz}$. Our main results are

- Hundreds of molecular lines reveal a rich chemistry, with molecules such as $\mathrm{HCN}$ and $\mathrm{HC}_{3} \mathrm{~N}$ especially abundant at high temperatures. Vibrationally excited $\mathrm{HCN}$, whose levels lie more than $1000 \mathrm{~K}$ above ground, is very optically thick, and even vibrationally excited $\mathrm{HC}^{15} \mathrm{~N}$ shows up at $690 \mathrm{GHz}$.

- Blue-shifted absorption in a dozen lines indicates an outflow oriented roughly along the line-of-sight. It is embedded as there is also absorption at the systemic velocity of cold foreground gas in $\mathrm{CO}$ and HCN.

- The averaged radial profile of the submm continuum displays a central flattening and rapid falloff, and is best fitted by a Plummer profile of the density. The mass of the source is on the order of several thousand $\mathrm{M}_{\odot}$, of which a few hundred are at high ( $\left.>300 \mathrm{~K}\right)$ temperatures.

- No fragmentation is observed over the $\sim 10$ beam sizes (30,000 AU) of the continuum emission. Highexcitation lines which are very optically thick do not show self-absorption. The line modeling suggests that this must be due to density fluctuations in combination with the velocity field. If the rather low continuum at $690 \mathrm{GHz}$ is real, it points to small, very dense condensations.

From these findings, a picture emerges of a very young forming star cluster, characterized by beginning of feedback from massive stars, while infall is ongoing. This feedback includes heating of the dust and gas, ionization that is still confined to small regions, and increased pressure in the inner part that leads to expansion motions and central flattening of the density.

\section{Acknowledgements}

We are grateful to Mark Gurwell for his help with the reduction of the $690 \mathrm{GHz}$ data, and to Kees Dullemond for kindly providing us with the RADMC-3D code. R. Rolffs acknowledges support from the International Max Planck Research School (IMPRS) for Astronomy and Astrophysics. 


\subsection{Appendix: Line maps}

In Figs. 6.18, 6.19, and 6.20, we show more integrated line maps, ordered by molecule as in Table 6.3 and by frequency of the transition (see also Sect. 6.5.3). 

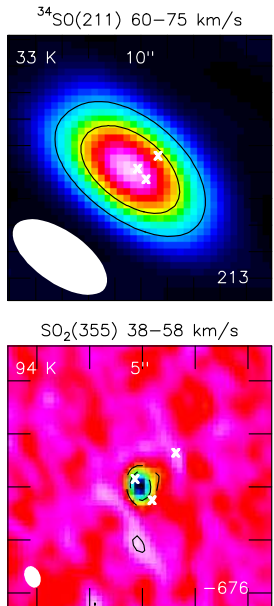

$\mathrm{CN}(680.3) 28-40 \mathrm{~km} / \mathrm{s}$
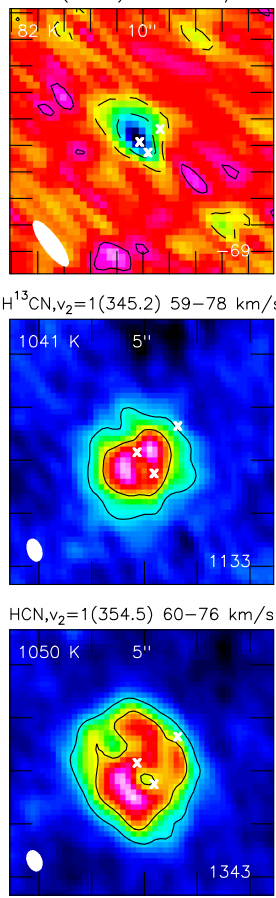

$75-84 \mathrm{~km} / \mathrm{s}$

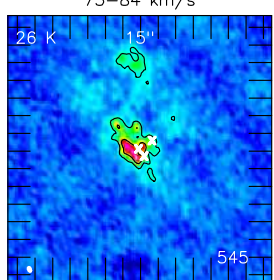

$H^{15} \mathrm{NC}(355.4) 59-72 \mathrm{~km} / \mathrm{s}$

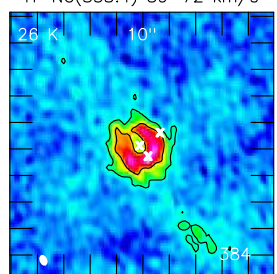

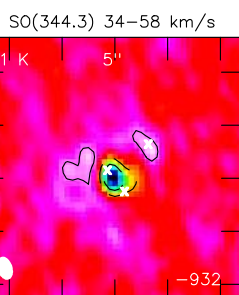

$59-76 \mathrm{~km} / \mathrm{s}$

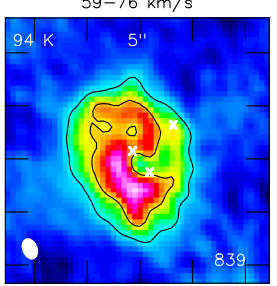

$47-61 \mathrm{~km} / \mathrm{s}$
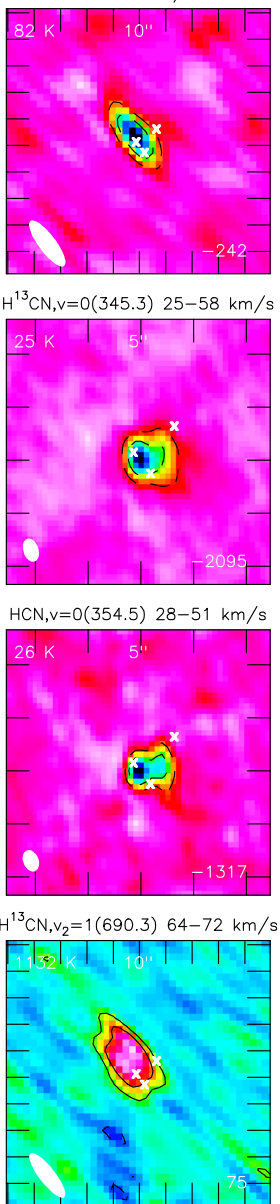

$\mathrm{CH}_{3} \mathrm{NH}_{2}(354.8) 59-73 \mathrm{~km} / \mathrm{s}$

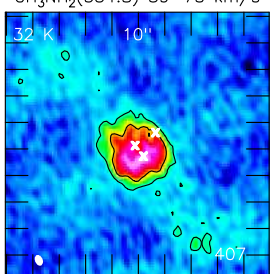

$58-68 \mathrm{~km} / \mathrm{s}$

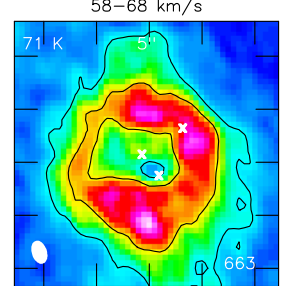

$\mathrm{SO}_{2}(681.7) 62-72 \mathrm{~km} / \mathrm{s}$

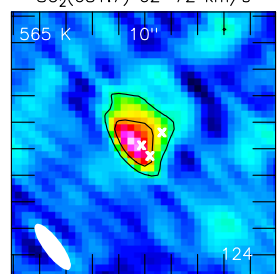

$H C^{15} \mathrm{~N}, \mathrm{v}=0(344.2) 45-58 \mathrm{~km} / \mathrm{s}$

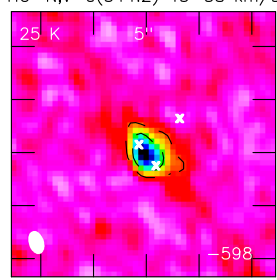

$58-64 \mathrm{~km} / \mathrm{s}$

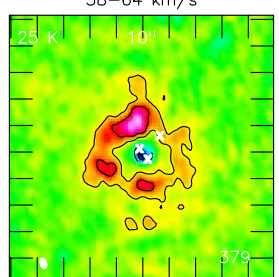

$52-64 \mathrm{~km} / \mathrm{s}$

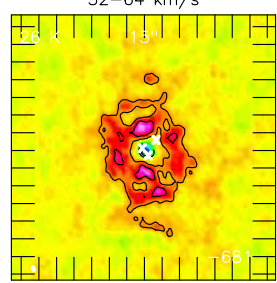

$H^{13} \mathrm{CN}, \mathrm{v}=\mathrm{O}(690.6) 39-65 \mathrm{~km} / \mathrm{s}$

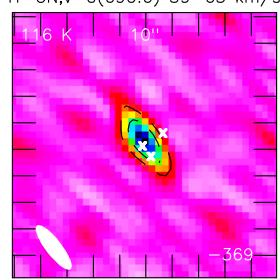

$\mathrm{CH}_{3} \mathrm{NH}_{2}(681.3) 59-72 \mathrm{~km} / \mathrm{s}$

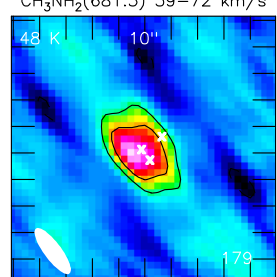

$68-74 \mathrm{~km} / \mathrm{s}$

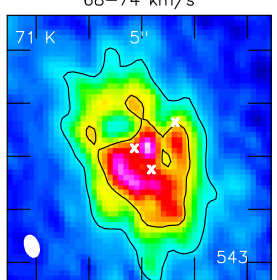

$\mathrm{OC}^{34} \mathrm{~S}(201.7) 58-78 \mathrm{~km} / \mathrm{s}$

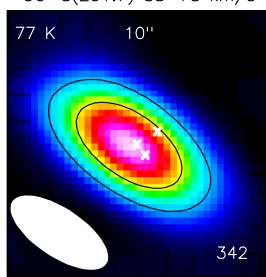

$58-68 \mathrm{~km} / \mathrm{s}$

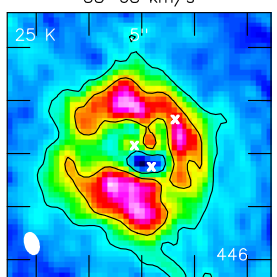

$64-69 \mathrm{~km} / \mathrm{s}$

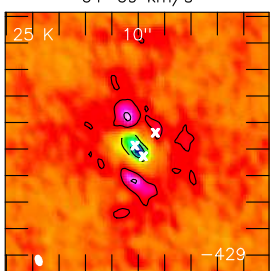

$64-70 \mathrm{~km} / \mathrm{s}$

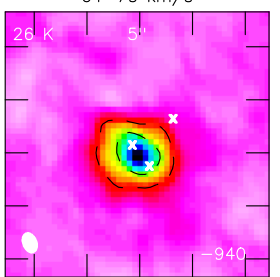

$66-72 \mathrm{~km} / \mathrm{s}$

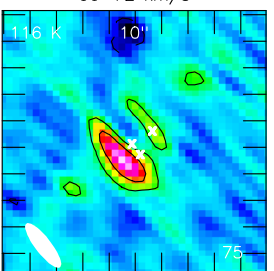

$\mathrm{CH}_{3} \mathrm{NC}(201) 58-75 \mathrm{~km} / \mathrm{s}$

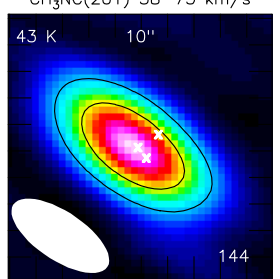

$74-81 \mathrm{~km} / \mathrm{s}$

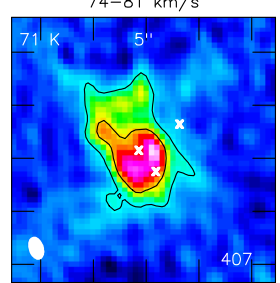

$0 C^{34} \mathrm{~s}(355.9) 60-74 \mathrm{~km} / \mathrm{s}$

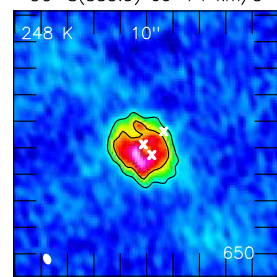

$68-78 \mathrm{~km} / \mathrm{s}$

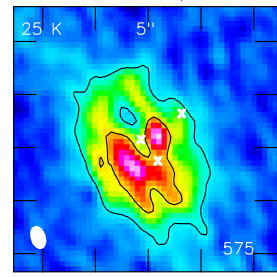

$69-73 \mathrm{~km} / \mathrm{s}$

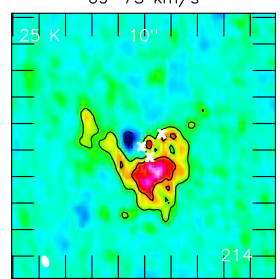

$71-74 \mathrm{~km} / \mathrm{s}$

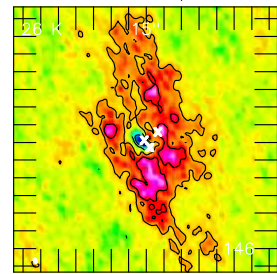

$H C^{15} \mathrm{~N}, \mathrm{v}_{2}=1(691.6) 63-73 \mathrm{~km} / \mathrm{s}$

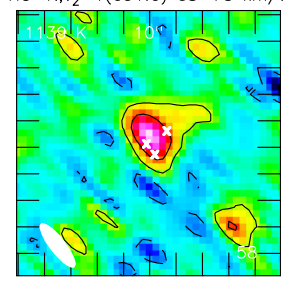

$\mathrm{NH}_{2} \mathrm{CN}(199.9) 61-71 \mathrm{~km} / \mathrm{s}$

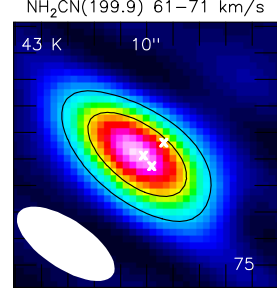

Figure 6.18: Line maps integrated over the range indicated in each panel. The map size is either 5, 10, or 15", as written in the map (tick spaces are 1", centered on R.A. 18:08:38.236, Dec. -19:51:50.25). Beams are shown in the lower left, and the number in the lower right of each panel is the maximum flux in $\mathrm{K} \mathrm{km} \mathrm{s}^{-1}$ (the contours are \pm 20 and $50 \%$ of that value). The color scale ranges from the minimum to the maximum value. The energy of the lower level is given in the upper left. 

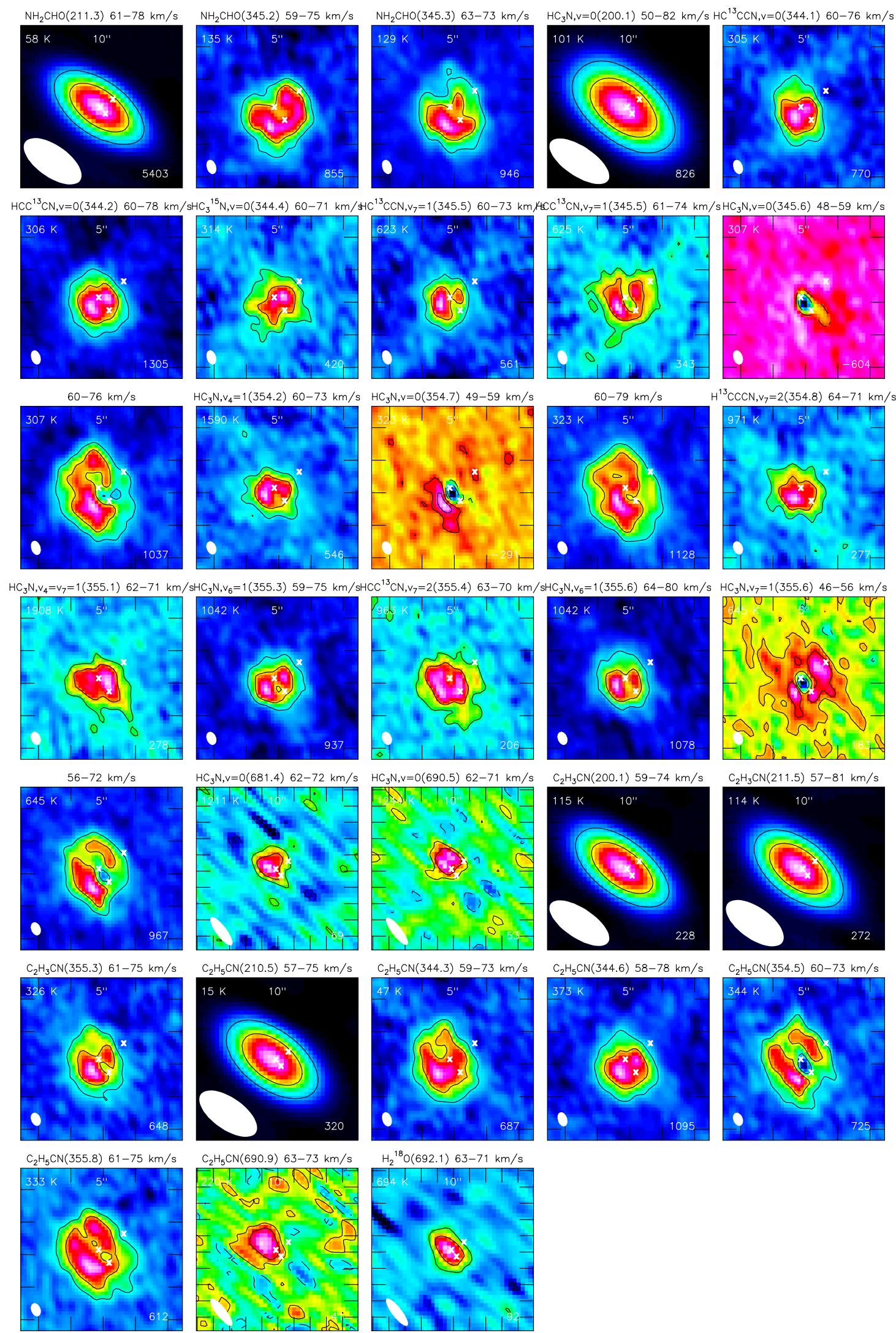

Figure 6.19: As Fig. 6.18, contd. 

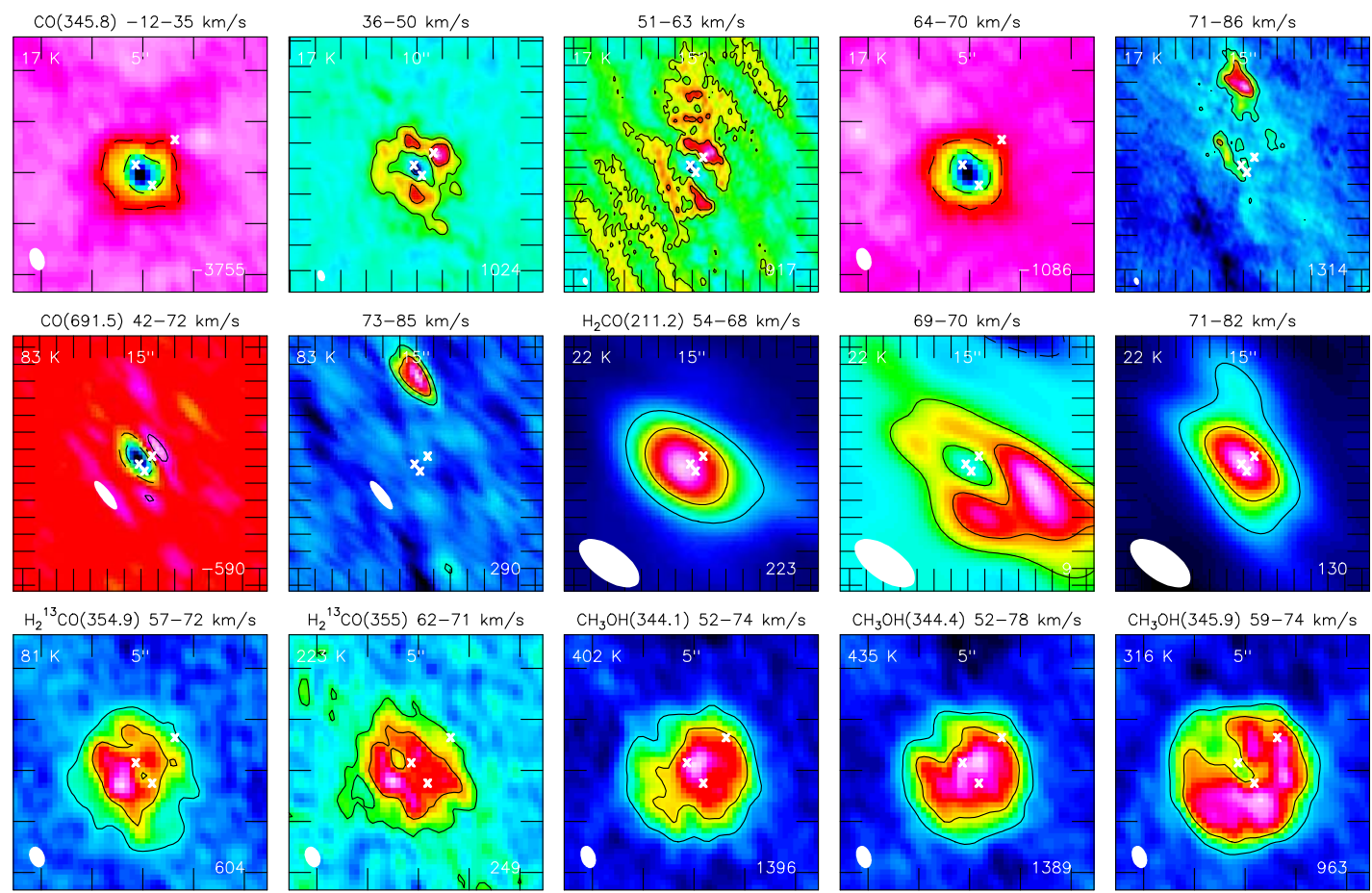

$\mathrm{CH}_{3} \mathrm{OH}(344.1) 52-74 \mathrm{~km} / \mathrm{s}$

$\mathrm{CH}_{3} \mathrm{OH}(344.4) 52-78 \mathrm{~km} / \mathrm{s}$

$\mathrm{CH}_{3} \mathrm{OH}(345.9) 59-74 \mathrm{~km} / \mathrm{s}$
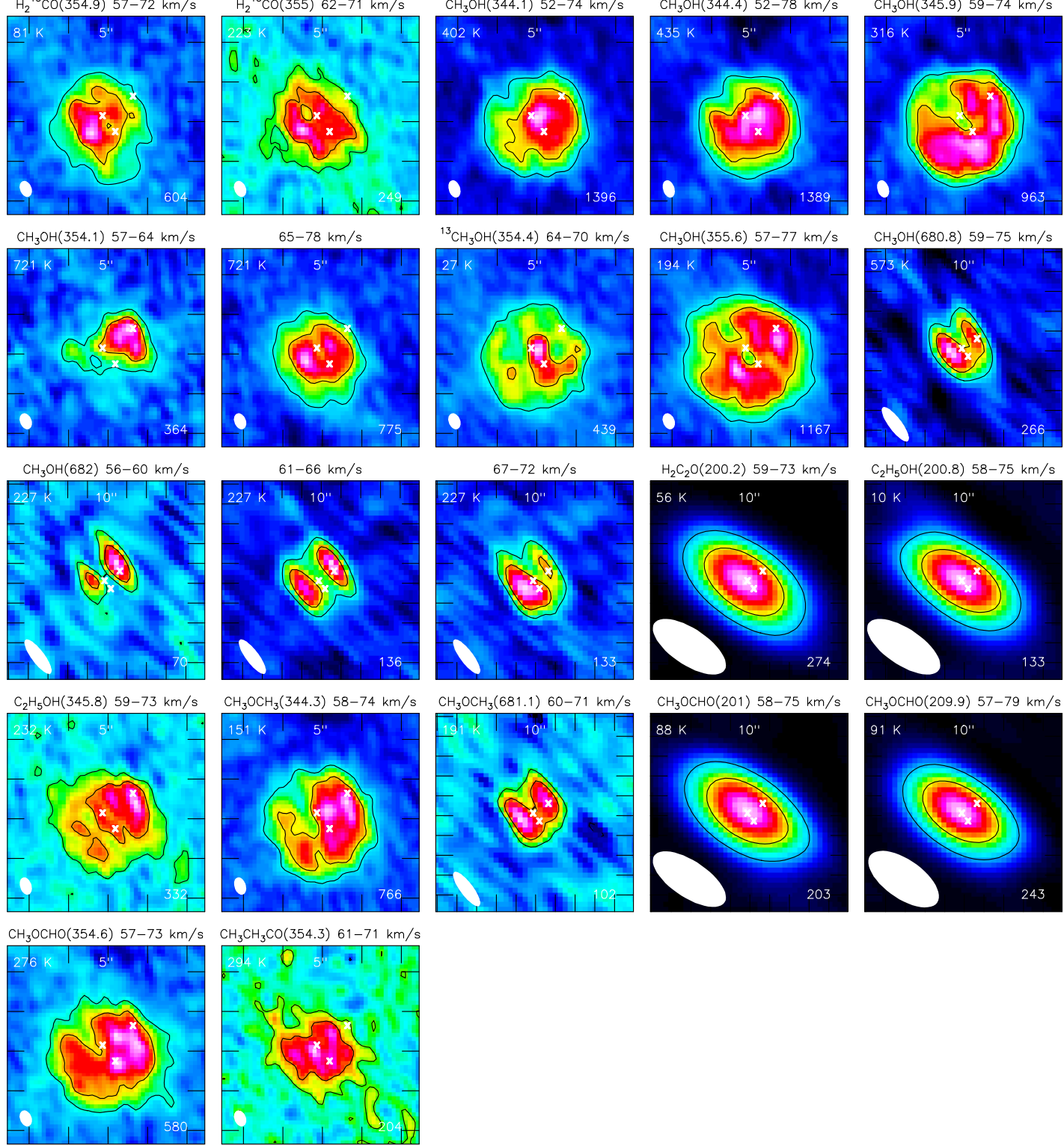

Figure 6.20: As Fig. 6.18, contd. 


\section{Chapter 7}

\section{Additional SMA paper: Submillimeter Continuum Observations of Sagittarius B2 at Subarcsecond Spatial Resolution}

A similar data set as for G10.47+0.03 has been obtained with the SMA for SgrB2-M and -N in 2010. The line data are not yet analyzed, but an $A \mathcal{E} A$ letter on the continuum is submitted: Qin, S. L., Schilke, P., Rolffs, R., Comito,

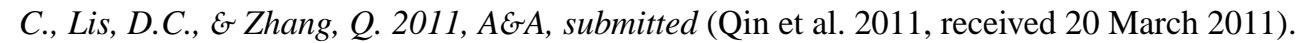

While SgrB2-M can be decomposed into twelve cores, SgrB2-N shows only two components, one northern core and one around K2, which does not fragment on the scales traced by the beam (3000 AU). This resembles $\mathrm{G} 10.47+0.03$, and also the radial profile and the continuum modeling that I performed for SgrB2-N are similar. The whole paper is reproduced in the following, but I wrote only Sect. 7.5.

\subsection{Abstract}

We report the first high spatial resolution submillimeter continuum observations of the Sagittarius B2 cloud complex using the Submillimeter Array (SMA). With the subarcsecond resolution provided by the SMA, the two massive star forming clumps Sgr B2(N) and Sgr B2(M) are resolved into multiple compact sources. In total, twelve submillimeter cores are identified in the Sgr B2(M) region, while only two components are observed in the Sgr B2(N) clump. The gas mass and column density are estimated from the dust continuum emission. We find that most of the cores have gas masses in excess of $100 \mathrm{M}_{\odot}$ and column densities above $10^{25} \mathrm{~cm}^{-2}$. The very fragmented appearance of Sgr B2(M), in contrast to the monolithic structure of Sgr B2 (N), suggests that the former is more evolved. The density profile of the Sgr B2(N)-SMA1 core is well fitted by a Plummer density distribution. This would lead one to believe that in the evolutionaly sequence of the Sgr B2 cloud complex, a massive star forms first in a homogeneous core, and the rest of the cluster forms subsequently in the then fragmenting structure.

\subsection{Introduction}

The Sagittarius B2 star-forming region is located $\sim 100 \mathrm{pc}$ from Sgr A*, within the $\sim 400 \mathrm{pc}$ wide dense Central Molecular Zone (CMZ) of the Galactic center, at a distance of $\sim 8 \mathrm{kpc}$ from the Sun (Reid et al. 2009). It is the strongest submillimeter continuum source in the CMZ (Schuller et al. 2009). It contains dense cores, Sgr B2(N) and Sgr B2(M), hosting clusters of compact Hir regions (Gaume et al. 1995; de Pree et al. 1998). It has been suggested that these two hot cores are at different evolutionary stages (Reid et al. 2009; Lis et al. 1993; Hollis et al. 2003; Qin et al. 2008). Spectral observations in centimeter and millimeter regimes have been conducted towards Sgr B2 (e.g. Carlstrom \& Vogel 1989; Mehringer \& Menten 1997; Nummelin et al. 1998; Liu \& Snyder 1999; Hollis et al. 2003; Friedel et al. 2004; Jones et al. 2008; Belloche et al. 2008), suggesting that Sgr B2(N) is chemically more active. Nearly half of all known interstellar molecules were first identified in Sgr B2(N), although sulphur-bearing molecules are more abundant in Sgr B2(M) than in Sgr B2(N).

The differences between Sgr B2(N) and Sgr B2(M), both in kinematics and chemistry, may originate in different physical conditions and thus different chemical history, or may simply be an evolutionary effect. A better understanding of the small-scale source structure and the exact origin of the molecular line emission is needed to distinguish between these two possibilities. In this Letter, we present high spatial resolution submillimeter 
continuum observations of Sgr B2(N) and Sgr B2(M), using the SMA ${ }^{1}$. The observations presented here resolve both Sgr B2 clumps into multiple submillimeter components. Together with the SMA spectral line data cubes and ongoing Herschel/HIFI complete spectral surveys towards Sgr B2(N) and Sgr B2(M) in the HEXOS key project, these observations will help in answering fundamental questions about the chemical ccomposition and physical conditions in Sgr B2(N) and Sgr B2(M).

\subsection{Observations}

The SMA observations of Sgr B2 presented here were carried out using seven antennas in the compact configuration on 2010 June 11, and using eight antennas in the very extended configuration on 2010 July 11 . The phase tracking centers were $\alpha(\mathrm{J} 2000.0)=17^{\mathrm{h}} 47^{\mathrm{m}} 19.883^{\mathrm{s}}, \delta(\mathrm{J} 2000.0)=-28^{\circ} 22^{\prime} 18.4^{\prime \prime}$ for Sgr B2(N) and $\alpha(\mathrm{J} 2000.0)=17^{\mathrm{h}} 47^{\mathrm{m}} 20.158^{\mathrm{s}}, \delta(\mathrm{J} 2000.0)=-28^{\circ} 23^{\prime} 05.0^{\prime \prime}$ for Sgr B2(M). Both tracks were observed in doublebandwidth mode with a $4 \mathrm{GHz}$ bandwidth in each of the lower sideband (LSB) and upper sideband (USB). The spectral resolution was $0.8125 \mathrm{MHz}$ per channel, corresponding to a velocity resolution of $\sim 0.7 \mathrm{~km} \mathrm{~s}^{-1}$. The observations covered rest frequencies from 342.2 to $346.2 \mathrm{GHz}$ (LSB), and from 354.2 to $358.2 \mathrm{GHz}$ (USB). QSOs 1733-130 and 1924-292 were evenly interleaved with the array pointings toward Sgr B2(N) and Sgr B2(M) during the observations in both configurations, for antenna gain calibration.

For the compact configuration observations, the typical system temperature was $273 \mathrm{~K}$. Mars, 3c454.3 and $3 \mathrm{c} 279$ were observed for bandpass calibration. The flux calibration was based on the observations of Neptune $\left(\sim 1.1^{\prime \prime}\right)$. For the very extended array observations, the typical system temperature was 292 K. 3c454.3 and 3c279 were used for bandpass calibration, and Uranus $\left(\sim 1.7^{\prime \prime}\right)$ was used for flux calibration. The absolute flux scale is estimated to be accurate to within $20 \%$.

The calibration and imaging were performed in Miriad (Sault et al. 1995). We note that there are spectralwindow-based bandpass errors in both amplitude and phase on some baselines in the compact array data, which have been corrected by use of a bright point source using the BLCAL task. The system temperature measurements for antennas 2 and 7 in the very extended array data were not recorded properly and have been corrected using the SMAFIX task. We selected line free channels as done by Qin et al. (2008). The continuum images were constructed from those, combining the LSB and USB data of both the compact and very extended array observations. We performed a self-calibration on the continuum data using the model from 'CLEANed' components for a few iterations in order to remove residual errors. Using the model from 'CLEANed' components, the self-calibration in our case did not introduce errors in the source structure, but improved the image quality and minimized the gain calibration errors. The final images were corrected for the primary beam response. The projected baselines ranged from 9 to $80 \mathrm{k} \lambda$ in the compact configuration and from 27 to $590 \mathrm{k} \lambda$ in the very extended configuration. The resulting synthesized beam is $0 .{ }^{\prime \prime} 4 \times 00^{\prime \prime} 24\left(\mathrm{PA}=14.4^{\circ}\right)$ using uniform weighting, and the $1 \sigma$ rms noise levels are 21 and $31 \mathrm{mJy}$ for Sgr B2(M) and Sgr B2(N) images, respectively. The difference in rms noise is caused by having more line free channels for continuum images in Sgr B2(M) (2417 channels) than in Sgr B2(N) (740 channels). Since there are no systematic offsets between the submm and $\mathrm{cm}$ sources, we believe the absolute astrometry to be good to $0.1^{\prime \prime}$.

\subsection{Results}

Continuum images of Sgr B2(N) and Sgr B2(M) at $850 \mu \mathrm{m}$ are shown in Fig. 7.1. Multiple submillimeter continuum cores are clearly detected and resolved towards both Sgr B2(M) and Sgr B2(N) with a spatial resolution of $0 . " 4 \times 0, ' 24$. Unlike in radio observations at $1.3 \mathrm{~cm}$ with a comparable resolution, which detected the UC HII regions K1, K2, K3, and K4 (Gaume et al. 1995), only two submillimeter continuum sources, SMA1 and SMA2, are observed in Sgr B2(N) (Fig. 7.1, upper panel). The bright component Sgr B2(N)-SMA1 is situated close to the UC HII region K2, and Sgr B2(N)-SMA2 is located $5^{\prime \prime}$ north of Sgr B2(N)-SMA1. The observations have shown that large saturated molecules only exist within a small region $\left(<5^{\prime \prime}\right)$ of Sgr B2(N) called the Large Molecule Heimat, Sgr B2(N-LMH) (Snyder et al. 1994). Our observations indicate that Sgr B2(N-LMH) coincides with Sgr B2(N)-SMA. Sgr B2(N)-SMA2 has also been detected in continuum emission at $7 \mathrm{~mm}$ and $3 \mathrm{~mm}$ (Rolffs et al. 2011a; Liu \& Snyder 1999) and molecular lines of $\mathrm{CH}_{3} \mathrm{OH}$ and $\mathrm{C}_{2} \mathrm{H}_{5} \mathrm{CN}$ at $7 \mathrm{~mm}$ (Mehringer \& Menten 1997; Hollis et al. 2003). Lower resolution continuum observations at $1.3 \mathrm{~mm}$ (see note to Table 1 of Qin et al. 2008) suggested that the Sgr B2(N)K1-K3 clump could not be fitted with a single Gaussian component, and another component existed at Sgr B2(N)-SMA2 position. Our observations here have resolved out Sgr B2(N)-SMA2, confirming it to be a high-mass core.

\footnotetext{
${ }^{1}$ The Submillimeter Array is a joint project between the Smithsonian Astrophysical Observatory and the Academia Sinica Institute of Astronomy and Astrophysics, and is funded by the Smithsonian Institution and the Academia Sinica.
} 
Table 7.1: Properties of the Continuum Sources

\begin{tabular}{|c|c|c|c|c|c|c|c|}
\hline Source & $\alpha(\mathrm{J} 2000.0)$ & $\delta(\mathrm{J} 2000.0)$ & Deconvolved size & $\begin{array}{r}\text { Peak Intensity } \\
\left(\mathrm{Jy} \mathrm{beam}^{-1}\right)\end{array}$ & $\begin{array}{c}\text { Flux Density } \\
(\mathrm{Jy})\end{array}$ & $\begin{array}{c}M_{\mathrm{H}_{2}} \\
\left(10^{2} \mathrm{M}_{\odot}\right)\end{array}$ & $\begin{array}{c}N_{\mathrm{H}_{2}} \\
\left(10^{25} \mathrm{~cm}^{-2}\right)\end{array}$ \\
\hline Sgr B2(N)-SMA1 & 174719.889 & -282218.22 & $1{ }^{\prime \prime} 72 \times 1{ }^{\prime \prime} 28\left(-7.7^{\circ}\right)$ & $1.79 \pm 0.039$ & $47.48 \pm 1.034$ & $27.31 \pm 0.59$ & $4.54 \pm 0.1$ \\
\hline Sgr B2(N)-SMA2 & 174719.885 & -282213.29 & $1 .^{\prime \prime} 44 \times 1 \prime^{\prime \prime} 02\left(-24.5^{\circ}\right)$ & $0.601 \pm 0.031$ & $10.12 \pm 0.521$ & $5.82 \pm 0.38$ & $1.45 \pm 0.1$ \\
\hline Sgr B2(M)-SMA1 & 174720.157 & -282304.53 & $1 \prime^{\prime \prime} 51 \times 00^{\prime \prime} 59\left(10.6^{\circ}\right)$ & $2.39 \pm 0.138$ & $20.9 \pm 1.21$ & $12.02 \pm 0.7$ & $4.94 \pm 0.29$ \\
\hline Sgr B2(M)-SMA2 & 174720.133 & -282304.06 & $0 .^{\prime \prime} 98 \times 00^{\prime \prime} 58\left(14.7^{\circ}\right)$ & $1.75 \pm 0.12$ & $12.51 \pm 0.859$ & $7.19 \pm 0.49$ & $4.63 \pm 0.32$ \\
\hline Sgr B2(M)-SMA3 & 174720.098 & -282303.9 & $0 .{ }^{\prime \prime} 93 \times 0 .{ }^{\prime \prime} 3\left(-9.9^{\circ}\right)$ & $0.866 \pm 0.09$ & $4.147 \pm 0.431$ & $2.38 \pm 0.25$ & $3.13 \pm 0.33$ \\
\hline Sgr B2(M)-SMA4 & 174720.150 & -282303.26 & $0 .^{\prime \prime} 55 \times 00^{\prime \prime} 32\left(-1.5^{\circ}\right)$ & $0.483 \pm 0.021$ & $1.443 \pm 0.063$ & $0.83 \pm 0.04$ & $1.73 \pm 0.08$ \\
\hline Sgr B2(M)-SMA5 & 174720.205 & -282304.78 & $00^{\prime \prime} 66 \times 0^{\prime \prime} 52\left(15.9^{\circ}\right)$ & $0.52 \pm 0.051$ & $2.488 \pm 0.244$ & $1.43 \pm 0.14$ & $1.53 \pm 0.15$ \\
\hline Sgr B2(M)-SMA6 & 174720.171 & -282305.91 & $0 . \prime 75 \times 0.0^{\prime \prime} 43\left(-24.3^{\circ}\right)$ & $0.71 \pm 0.067$ & $3.05 \pm 0.288$ & $1.75 \pm 0.17$ & $1.99 \pm 0.19$ \\
\hline Sgr B2(M)-SMA7 & 174720.11 & -282306.18 & $0 . \prime 72 \times 0 . .37\left(14.9^{\circ}\right)$ & $0.6 \pm 0.051$ & $2.366 \pm 0.201$ & $1.36 \pm 0.12$ & $1.87 \pm 0.16$ \\
\hline Sgr B2(M)-SMA8 & 174720.216 & -282306.48 & $0 . \prime 52 \times 0 . \prime 45\left(-63.4^{\circ}\right)$ & $0.31 \pm 0.048$ & $1.246 \pm 0.193$ & $0.71 \pm 0.11$ & $1.12 \pm 0.17$ \\
\hline Sgr B2(M)-SMA9 & 174720.238 & -282306.87 & $0 . \prime 83 \times 0 . \prime 42\left(23.9^{\circ}\right)$ & $0.369 \pm 0.032$ & $1.922 \pm 0.164$ & $1.11 \pm 0.09$ & $1.16 \pm 0.1$ \\
\hline Sgr B2(M)-SMA10 & 174719.989 & -282305.83 & $3 .^{\prime \prime} 06 \times 1 .^{\prime \prime} 0\left(-25.8^{\circ}\right)$ & $0.183 \pm 0.015$ & $4.321 \pm 0.363$ & $2.49 \pm 0.29$ & $0.3 \pm 0.03$ \\
\hline Sgr B2(M)-SMA11 & 174720.106 & -282303.01 & $0 . \prime 97 \times 0 . " 48\left(-27.7^{\circ}\right)$ & $0.695 \pm 0.058$ & $4.423 \pm 0.37$ & $2.54 \pm 0.21$ & $2 \pm 0.17$ \\
\hline Sgr B2(M)-SMA12 & 174720.132 & -282302.24 & $0 \prime^{\prime \prime} 82 \times 0 .^{\prime \prime} 52\left(0.9^{\circ}\right)$ & $0.394 \pm 0.018$ & $2.629 \pm 0.121$ & $1.51 \pm 0.08$ & $1.3 \pm 0.07$ \\
\hline
\end{tabular}

Notes. Units of right ascension are hours, minutes, and seconds, and units of declination are degrees, arcminutes, and arcseconds.

The continuum image of Sgr B2(M) (Fig. 7.1, lower panel) shows a complicated morphology, with a roughly north-south extending envelope encompassing several compact components. In total, twelve submillimeter sources are resolved in Sgr B2(M). Using the Very Large Array (VLA), Gaume et al. (1995) have detected four bright UC HII regions (F1-F4) at $1.3 \mathrm{~cm}$ within 2" in Sgr B2(M). The highest resolution $7 \mathrm{~mm}$ VLA image with a position accuracy of 0.1" (de Pree et al. 1998) has resolved nineteen UC HII regions in the central region of Sgr B2(M)(F1F4). Five submillimeter components, Sgr B2(M)-SMA1 to SMA5 are detected in the central region of Sgr B2(M) in our observations. Outside of the central region, seven components, Sgr B2(M)-SMA6 to SMA12 are identified. Given the positional accuracies of our observations and the observations by de Pree et al. (1998), the projected positions of Sgr B2(M)-SMA2, SMA6, SMA11 and SMA12 coincide with those of UC HII regions F1, F10.37, F10.30 and F10.318, respectively. No centimeter source is detected towards Sgr B2(M)-SMA10, located southwest of the central region, which shows extended structure.

Multi-component Gaussian fits were carried out towards both the Sgr B2(N) and Sgr B2 (M) clumps using the IMFIT task. The residual fluxes after fitting are 4 and $11 \mathrm{Jy}$ for Sgr B2(N) and Sgr B2(M), respectively. The large residual error in Sgr B2(M) clump is most likely caused by its complicated source structure. The peak positions, deconvolved angular sizes (FWHM), peak intensities, and total flux densities of the continuum components are summarized in Table 7.1. The total flux densities of the Sgr B2(N) and Sgr B2 (M) cores are 58 and $61 \mathrm{Jy}$, while the peak fluxes measured by the bolometer array LABOCA at the 12-m telescope APEX are 150 and 138 Jy in a $18.2^{\prime \prime}$ beam respectively (Schuller, priv. comm.), indicating that $\sim 60 \%$ flux is filtered out and our SMA observations only pick up the densest parts of the Sgr B2 cores.

Assuming that the $850 \mu \mathrm{m}$ continuum is due to optically thin dust emission and using an average grain radius of $0.1 \mu \mathrm{m}$, grain density of $3 \mathrm{~g} \mathrm{~cm}^{-3}$ and a gas to dust ratio of 100 (Hildebrand 1983; Lis et al. 1991), the mass and column density can be calculated using the formulae given in Lis et al. (1991). We adopt $Q(v)=4 \times 10^{-5}$ at 850 $\mu \mathrm{m}$ (Hildebrand 1983; Lis et al. 1991) and a dust temperature of $150 \mathrm{~K}$ (Carlstrom \& Vogel 1989; Lis et al. 1993) in the calculation. Based on flux densities at $1.3 \mathrm{~cm}$ and $7 \mathrm{~mm}$ (Gaume et al. 1995; de Pree et al. 1998; Rolffs et al. 2011a), most of $\mathrm{K}$ and $\mathrm{F}$ subcomponents (except for $\mathrm{K} 2$ and F3) at $7 \mathrm{~mm}$ have fluxes less than or comparable with those at $22.4 \mathrm{GHz}$, showing descending spectra and suggesting optically thin HII regions at short wavelengths. The contributions of the free-free emission to the flux densities of the submillimeter components are less than $0.7 \%$ for $\mathrm{K} 2$ and $\mathrm{F} 3$ and less than $0.1 \%$ for other components and can safely be ignored. The estimated clump masses and column densities are given in Table 7.1. The flux density of the fourteen detected submillimeter components ranges from 1.2 to $47 \mathrm{Jy}$, corresponding to gas masses from 71 to $2731 M_{\odot}$. The column densities are a few times $10^{25}$ $\mathrm{cm}^{-2}$. Under the Rayleigh-Jeans approximation, $1 \mathrm{Jy} \mathrm{beam}^{-1}$ in our SMA observations corresponds to a brightness temperature of $113 \mathrm{~K}$. The peak brightness temperatures of Sgr B2(M)-SMA1 and Sgr B2(N)-SMA1 are 270 and $200 \mathrm{~K}$ respectively, which give a lower limit to the dust temperatures at the peak position of the continuum. In this paper, the column densities and masses are estimated by use of source-averaged continuum fluxes. Based on the model fitting (Lis et al. 1991, 1993), the adopted $Q(v)$, dust temperature, and optically thin approximation are reasonable guesses for the Sgr B2(N) and Sgr B2(M) cloud complexes. The total masses of Sgr B2(N) and (M), determined by summing up over the components, are very comparable, 3313 and $3532 \mathrm{M}_{\odot}$, respectively, in spite of very different morphologies. The adopted gas temperature of $150 \mathrm{~K}$ we consider a reasonable guess for the 
average core temperatures, although the most massive cores show higher peak brightness temperatures. For those, the optically thin assumption also is probably not justified, and we would be underestimating their masses.

\subsection{Modeling}

A striking feature in the maps is the appearance of Sgr B2(N)-SMA1 which, although well resolved, does not appear to be fragmented. We used the three-dimensional radiative-transfer code RADMC-3D ${ }^{2}$, developed by C. Dullemond, to model the continuum emission of Sgr B2(N)-SMA1. Figure 7.2 shows three example models, whose radial profiles were obtained by Fourier-transforming the computed dust continuum maps, 'observing' with the uv coverage of the data, and averaging the image in circular annuli. All models are heated by the stars in the UC HII regions $\mathrm{K} 2$ and $\mathrm{K} 3$, assumed to have luminosities of $\approx 10^{5} \mathrm{~L}_{\odot}$, which is uncertain, but should give the right order of magnitude (Rolffs et al. 2011a). The dust mass opacity $\left(0.6 \mathrm{~cm}^{2} \mathrm{~g}^{-1}\right)$ is interpolated from Ossenkopf \& Henning (1994) without grain mantles or coagulation, which corresponds to a $Q(v)$ of a few times $10^{-5}$ at $850 \mu \mathrm{m}$ and is consistent with the value used in our calculation of masses and column densities. The model with a density distribution that follows the Plummer profile, $n=1.7 \times 10^{8} \times\left(1+\left(\frac{r}{11500 \mathrm{AU}}\right)^{2}\right)^{-2.5} \mathrm{H}_{2} \mathrm{~cm}^{-3}$ (half-power radius 6500 AU) provides the best fit. Also shown in Fig. 7.2 are a Gaussian model with central density $2 \times 10^{8} \mathrm{H}_{2} \mathrm{~cm}^{-3}$ and half-power radius $6500 \mathrm{AU}$, and a model whose density follows a radial power law, $n=10^{9} \times\left(\frac{r}{1000 \mathrm{AU}}\right)^{-1.5} \mathrm{H}_{2} \mathrm{~cm}^{-3}$ (outside of $1000 \mathrm{AU}$, the radius of the HII region K2). The latter model reproduces the peak flux measured by the bolometer array LABOCA at the 12-m telescope APEX, which is $150 \mathrm{Jy}$ in a $18.2^{\prime \prime}$ beam, but does not fit the inner regions observed by the SMA very well. The Plummer and Gauss models fit Sgr B2(N)-SMA1 well, both in terms of shape and absolute flux. This implies the existence of an extended component, which is filtered out in the SMA map, but picked up by LABOCA.

Whether the Plummer profile, which is also used to describe density profiles of star clusters, has any relevance for an evolving star cluster, or whether the cluster loses its memory of the gas density profile in the subsequent dynamical evolution is not clear at this point. The model has a mass of around $3000 \mathrm{M}_{\odot}$ inside a radius of 11500 $\mathrm{AU}(0.056 \mathrm{pc})$, which gives an average density of $4 \times 10^{6} \mathrm{M}_{\odot} \mathrm{pc}^{-3}$. This is only the mass contained in the gas, and does not include the mass of the already formed compact objects providing the luminosity. Sgr B2(N) could be a very young, embedded stage of the formation of a massive star cluster.

\subsection{Discussion}

Our SMA observations resolve the Sgr B2(M) and (N) cores into fourteen compact submillimeter continuum components. The two cores display very different morphologies. The source Sgr B2(N)-SMA1 is located northeast of the centimeter source $\mathrm{K} 2$, with an offset of $\sim 0.2^{\prime \prime}$. The continuum observations at $1.3 \mathrm{~cm}$ with a resolution of $0.25^{\prime \prime}$ (Gaume et al. 1995) and at $7 \mathrm{~mm}$ with a resolution of 0.1" (Rolffs et al. 2011a) also showed a compact component centered at $\mathrm{K} 2$. We failed to detect any submillimeter continuum emission associated with sources $\mathrm{K} 1$, $\mathrm{K} 3$, and $\mathrm{K} 4$. Sgr B2(N)-SMA2 is a high-mass dust core.

In contrast to Sgr B2(N), a very fragmented cluster of high mass submillimeter sources is detected in Sgr B2(M). In addition to the two brightest and most massive components, Sgr B2(M)-SMA1 and Sgr B2(M)-SMA2, situated in the central region of Sgr B2(M), ten additional sources are detected, demonstrating a high degree of fragmentation. The sensitivity of $0.021 \mathrm{Jy} \mathrm{beam}^{-1}$ in our observations corresponds to a detectable gas mass of $1.2 \mathrm{M}_{\odot}$, but the observations are likely dynamic range limited, so it is difficult to determine the clump mass function in Sgr B2(M) down to smaller masses.

The estimated column densities $\left(10^{25} \mathrm{~cm}^{-2}=33.4 \mathrm{~g} \mathrm{~cm}^{-2}\right)$ in both the homogeneous starforming region Sgr B2(N) and the clustered Sgr B2(M) region are well in excess of the threshold of $1 \mathrm{~g} \mathrm{~cm}^{-2}$ for preventing cloud fragmentation and formation of massive stars (Krumholz \& McKee 2008). The source sizes and masses derived from Gaussian fitting, assuming a spherical source, lead to volume densities in excess of $10^{7} \mathrm{~cm}^{-3}$ for all submillimeter sources detected in the SMA images. Assuming a gas temperature of $150 \mathrm{~K}$, the thermal Jeans masses are less than $10 \mathrm{M}_{\odot}$, but the turbulent support is considerable. The large column densities, gas masses and the velocity field (Rolffs et al. 2010) suggest that the submillimeter components in the two regions are gravitationally unstable and are in the process of forming massive stars. This process seems more advanced in Sgr B2(M), which is also reflected in the large number of embedded UC HII regions found there. However, star formation in Sgr B2(N) appears not to have progressed very far, a conclusion also supported by the presence of only one UC HII region embedded in one of the two clumps studied here. The observations also showed that massive star formation taking

\footnotetext{
${ }^{2}$ http://www.ita.uni-heidelberg.de/ dullemond/software/radmc-3d
} 
place in the two clumps with outflow age of $\sim 10^{3}$ and $\sim 10^{4}$ year for Sgr B2(N) and Sgr B2(M) clumps, respectively (Lis et al. 1993). If one can generalize from these two examples, and if they provide snapshots in time of the evolution of basically equal cores, it seems that a massive star forms first in a relatively homogeneous core, another example with Plummer density profile and without fragmentation is the high-mass core G10.47 (Rolffs et al. 2011c), followed up by fragmentation or at least visible break-up of the core and subsequent star formation, perhaps aided by radiative or outflow feedback from the first star. This scenario may well apply to extremely highmass cluster forming cores or to special environments only, since high-mass IRDCs have been shown to fragment early (Zhang et al. 2009). 

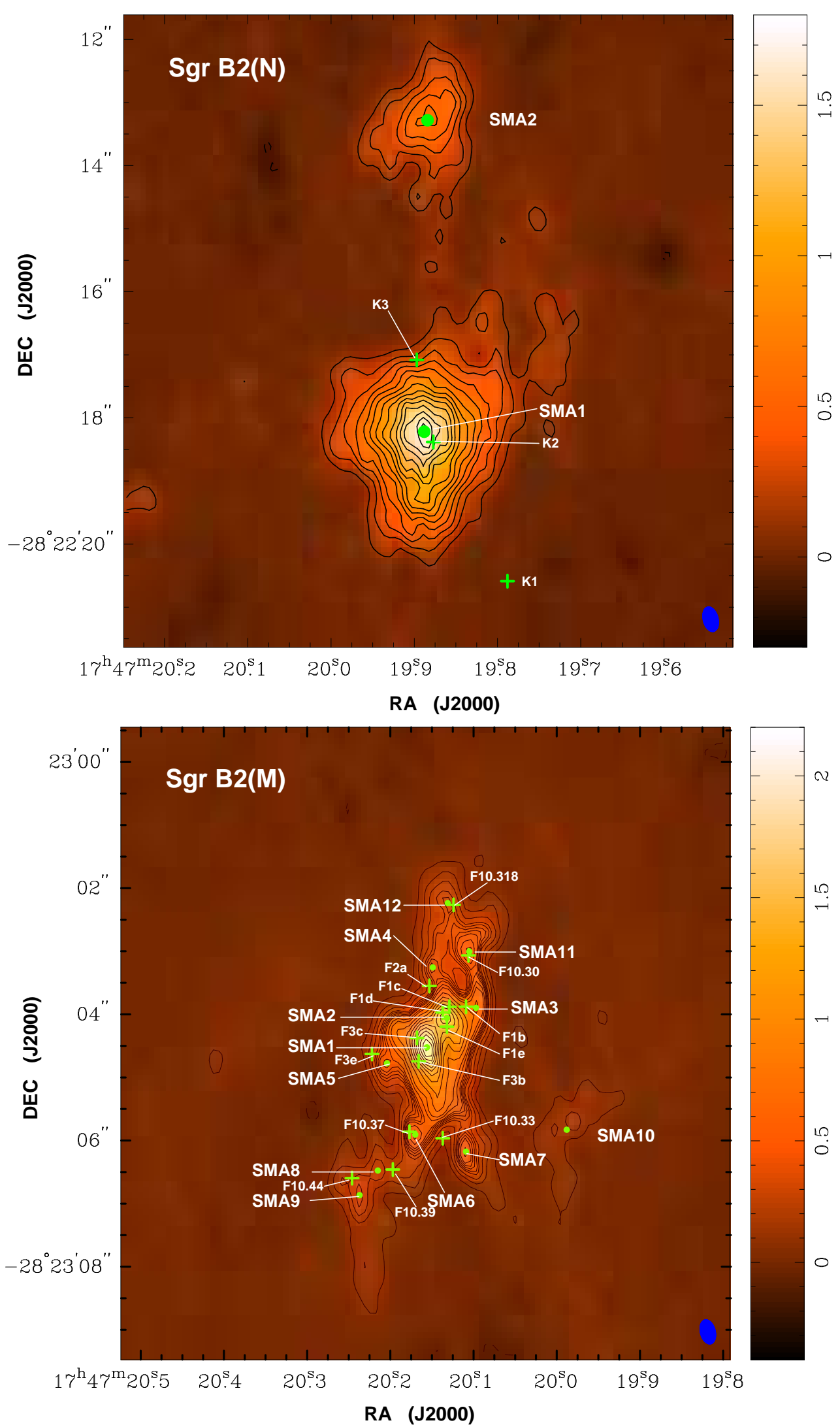

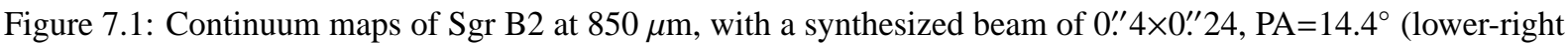
corner in each panel). The left panels shows the image of $\operatorname{Sgr} \mathrm{B} 2(\mathrm{~N})$, with contour levels $(-1,1, \ldots 14) \times 4 \sigma$ $\left(1 \sigma=0.031 \mathrm{Jy} \mathrm{beam}^{-1}\right)$. The cross symbols indicate the positions of UC HiI regions detected in $1.3 \mathrm{~cm}$ continuum (Gaume et al. 1995). The right panel present the image of Sgr B2(M), with contour levels $(-1,1,2,3,4,4.5,5.5,5$, $6,7,8,10, \ldots 28) \times 4 \sigma\left(1 \sigma=0.021 \mathrm{Jy} \mathrm{beam}^{-1}\right)$. The cross symbols indicate the positions of UC HII regions detected in $7 \mathrm{~mm}$ continuum (de Pree et al. 1998). In each panel, the filled circle symbols present the peak positions of the submillimeter continuum sources. 


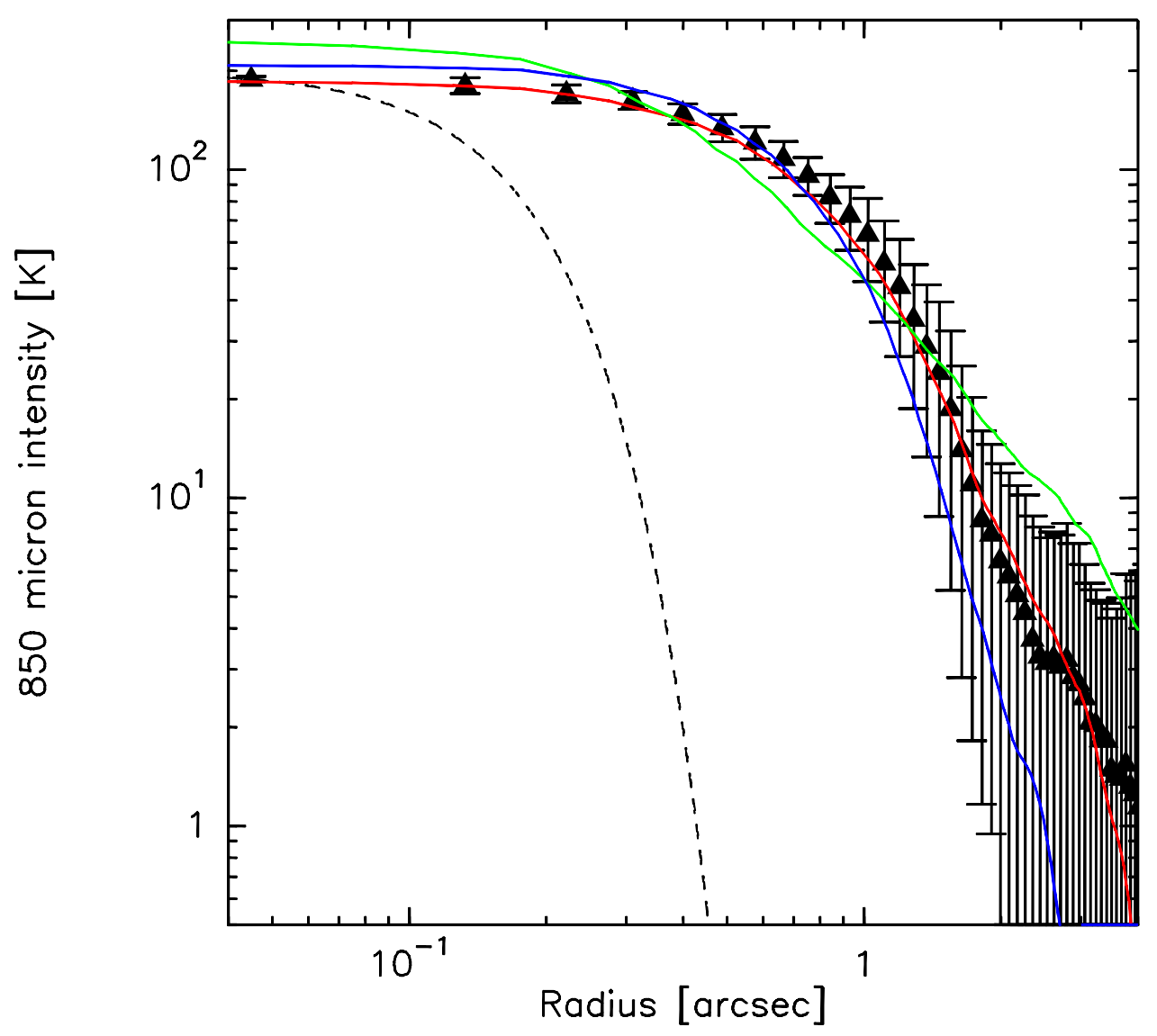

Figure 7.2: Radial profile of the Sgr B2(N)-SMA1 component, with errorbars denoting the rms in circular annuli. Overlaid are three models with different density distributions (red: Plummer; blue: Gaussian; green: power-law). The beam is depicted as a dashed Gaussian. 


\section{Chapter 8}

\section{Discussion}

In this chapter I summarize and discuss all results that were obtained from the different data sets and models. More details can be found in Sects. 3.9, 4.6, 5.7, and 6.7.

\subsection{Heating}

\subsubsection{Internal Heating}

Heating is internal if it is caused by heating sources which are deeply embedded inside the core, whereas external heating comes from outside the core, e.g. from UCHIr regions which are not surrounded by high column densities. The results obtained in this thesis support the internal heating of HMCs.

The single-dish observations reveal strong emission from vibrationally excited HCN in all sources and from vibrationally excited $\mathrm{H}^{13} \mathrm{CN}$ in half of the sources that were observed with APEX. In addition, other high-excitation lines such as from $\mathrm{HC}_{3} \mathrm{~N}$ or vibrationally excited $\mathrm{HNC}$ (in SgrB2-M with Herschel/HIFI) are detected. Especially vibrationally excited $\mathrm{H}^{13} \mathrm{CN}$ requires large masses of hot gas, which can hardly be provided without diffusion of radiation. Diffusion is photon-trapping by high dust column densities in all directions from the heating source, and increases the efficiency of heating dramatically (e.g. Kaufman et al. 1998).

The VLA data show the extension of vibrationally excited HCN, tracing hot dust. Emission around the HCHII regions and absorption against them clearly point to the stars in the HCHII regions as heating sources. Modeling of the dust temperature and the resulting line emission and absorption supports this heating, aided by large column densities that lead to diffusion.

The SMA data contain many optically thick high-excitation lines, whose emission coincides with the dust continuum. Moreover, lines from higher energy levels are generally more compact, indicating a centrally peaked temperature. Also the self-absorption features in the single-dish data require such a temperature gradient.

At least the three sources investigated by interferometers (G10.47+0.03, SgrB2-N and -M) and, most likely, the other three sources that show vibrationally excited $\mathrm{H}^{13} \mathrm{CN}$ in the APEX data (G327.3-0.6, IRAS 16065-5158, IRAS 17233-3606) must be internally heated. The situation is less clear for the remaining six sources that were observed with APEX, since larger scales are probed by this instrument and interferometer data are needed to clarify e.g. the location of vibrationally excited HCN. G34.26+0.15 is a case for external heating (Watt \& Mundy 1999; Mookerjea et al. 2007); it nevertheless shows strong vibrationally excited HCN and global infall, indicating ongoing star formation, which could heat the hot core from the inside in addition to the neighboring UCHII region.

Modes of internal heating are ultraviolet radiation from hot stars, infrared radiation from colder stars and from accretion, which releases energy close to the stars, as well as shocks and decaying turbulence, converting kinetic energy from winds and outflows into heat. The emission spectrum of embedded stars or protostars does not matter, since their radiation is reprocessed by dust. Only their luminosity is relevant, and the high luminosity of HMCs points to high-mass stars or star clusters/associations as the dominant heating source. For instance, external heating by X-ray radiation is responsible for the hot layer in the envelope of SgrB2 (Comito et al. 2003; Wilson et al. 2006), but can be excluded for HMCs already on the basis of this luminosity argument. Also cosmic rays are negligible compared to the internal sources.

\subsubsection{Multiplicity of Heating Sources}

The high-excitation and most other lines observed with the single-dish telescopes can be well explained by central heating, as assumed in the RATRAN modeling. However, the emission from high- $J$ lines in the outer pixels of $\mathrm{CHAMP}+$ could mean additional heating there. 
Most obvious is the multiplicity of heating sources in the VLA continuum maps, where many HII regions are detected. Especially SgrB2-M is very rich in HII regions, each ionized by a massive star. Also G10.47+0.03 and SgrB2-N have more than one Hir region, and the presence (or neighborhood) of multiple stars seems to be a general feature of HMCs.

While it is relatively easy to take these stars into account in the modeling with RADMC-3D, heating sources without detectable ionized gas are much more difficult to incorporate. Their locations are not evident, and even their existence is questionable. It is expected that a huge number exists, since massive stars as those visible in the HII regions do not form alone, but accompanied by many less massive stars. Several models were run with additional stars whose luminosities are distributed according to the initial luminosity function, but the results are ambiguous. In particular, the observational data could be reproduced as well with only the stars in the HII regions. It is possible that these are the first stars that have formed, and the rest will follow later.

On the other hand, the extension of high-excitation lines in the SMA maps is generally a bit smaller in the models than in the data, especially highly excited $\mathrm{HC}_{3} \mathrm{~N}$ is somewhat extended to the east (Fig. 6.5). More modeling efforts are needed to decide if this is an abundance/density effect or if more heating sources are necessary.

\subsection{Density Distribution}

\subsubsection{Radial Profile}

Dust continuum emission traces the density, modulated by temperature and dust opacity. Temperature gradients are taken into account in the modeling by the central heating (RATRAN) or the stars in the HII regions (RADMC-3D). Variations in opacity are much less than in density, and are neglected due to unknown opacity behavior and the degeneracy with density.

The averaged radial profile of the continuum, as measured with LABOCA on APEX, can be fitted by a powerlaw density distribution, with density proportional to radius to the power of about -1.5 . The LABOCA data have a resolution of $18.2^{\prime \prime}$, and therefore are sensitive only to large scales. This power-law density distribution fits also to most of the lines observed with APEX and Herschel.

The interferometer data, however, reveal that in the inner part the density does not follow a radial power-law profile, but is rather flat in a central region and falls off rapidly outside this region. The VLA line emission could be modeled with a Gaussian density profile and the dust emission seen by the SMA with a Plummer profile (which resembles a Gaussian in the center). Such centrally dense profiles could arise from increased pressure due to feedback from massive stars.

\subsubsection{Clumpiness}

The emission from high- $J$ lines in the outer pixels of the CHAMP+ receiver on APEX can only be explained by the presence of high densities at the location of the outer pixels (15-20" from the center). However, there cannot be such high densities everywhere at these radii, as the continuum measured with LABOCA would be much higher then. Therefore, the density has to vary, and high-density clumps in a lower-density environment are needed to explain the emission in the outer pixels. These clumps have to be warm enough to excite the high- $J$ levels, probably suggesting additional heating.

An independent hint on clumpiness comes from the line shapes. There is a lack of self-absorption in optically thick lines in some sources observed with APEX. These lines also do not show a central plateau, but are simply single-peaked. An explanation for such a shape would be the overlay of many optically thick profiles, each with a different velocity. These velocities would be associated with separate clumps.

Vibrationally excited $\mathrm{HCN}$ is very optically thick at least in those sources with vibrationally excited $\mathrm{H}^{13} \mathrm{CN}$. Nevertheless, it too does not display self-absorption features. For the APEX data, this can be explained by the large beam: Any absorption is reemitted well inside the beam, so no self-absorption can be observed. This does not apply for the SMA data, however, which resolves the emission. Modeling a homogeneous, internally heated core inevitably produces strong self-absorption in the optically thick lines. The situation improves significantly when the model is inhomogeneous, with density fluctuations (clumpiness) and varying velocity field. The line shapes can be much better reproduced with such models.

Direct imaging of the clumpy substructure could have been possible with the resolution of the VLA data. Indeed, the maps show strong variations of the emission, but the low signal-to-noise ratio in addition to possible phase noises makes it very unclear whether this is real. It is entirely possible that such variations arise simply from noise, so the degree of clumpiness cannot be decided on the basis of these data. The SMA data, with $\sim 3$ times lower resolution, do not show any fragmentation of line or continuum emission in the central region. 


\subsection{Velocity Field}

\subsubsection{Infall}

Blue asymmetries in self-absorbed lines are an indication of infall, as the absorption is red-shifted and the blueshifted emission from the far inner side can escape freely. In many of the sources observed with APEX this infall signature is detected, and could be modeled as a spherical infall with about $10 \%$ of the free-fall speed $\left(\sim 1 \mathrm{~km} \mathrm{~s}^{-1}\right)$. This means that large-scale accretion onto the hot core occurs, although with a lower speed than expected from unhindered contraction.

The reduced average infall speed is a hint on processes that counteract gravitational contraction, in particular turbulent support. Overall, gravity is still stronger, allowing the HMC to grow in mass.

\subsubsection{Expansion}

In many lines observed with APEX, high-velocity line wings are seen. This emission of fast gas likely originates in an outflow. Also self-absorbed line profiles with red asymmetries are present, which are indicative of expansion motions. In some sources, e.g. G327.3-0.6 and SgrB2-M, both red and blue asymmetries occur, putting in question whether the source is expanding or infalling.

This could be investigated with Herschel data of SgrB2-M, which include higher- $J$ transitions of HCN. The systematic change of the asymmetries from blue to red with increasing $J$ could be modeled as infall in the outer parts and expansion in the inner parts. Thus, the infall is reversed close to the protocluster due to the onset of feedback from the young stars.

More directly, blue-shifted absorption (meaning that foreground gas moves towards us) could be observed at high angular resolution with the VLA and the SMA. Velocity components of hot gas moving at $\sim 10 \mathrm{~km} \mathrm{~s}^{-1}$ away from the HCHII regions SgrB2-M F1f and G10.47+0.03 B1 and B2 are detected.

The SMA observations show such expansion motions for a variety of molecular lines in absorption against the dust continuum. Expansion velocities up to $\sim 40 \mathrm{~km} \mathrm{~s}^{-1}$ are seen, with the bulk moving at $\sim 18 \mathrm{~km} \mathrm{~s}^{-1}$. In spite of this outflow, spectra of lines with high optical depth such as $\mathrm{CO}, \mathrm{HCN}$, or $\mathrm{H}^{13} \mathrm{CN}$ display the infall asymmetry if integrated over a larger region. This means that blue-shifted emission from the far side is stronger than blue-shifted absorption at the near side, and that the outflow is confined to a small central region, while the gas is infalling on larger scales and with a higher rate, but at a lower velocity.

\subsubsection{Other Motions}

Turbulence, along with unresolved macroscopic motions, broadens the lines to widths of $3-10 \mathrm{~km} \mathrm{~s}^{-1}$. Different velocity components at different locations are not resolved with the single-dish observations, and might contribute to the line width and also to shifts in the line velocity that were observed with Herschel/HIFI. With the interferometer observations, velocity components in SgrB2-M could be clearly distinguished (F1 and F3).

Continuous velocity gradients can be an indication of rotation, but could also be due to unresolved components. The absorption towards SgrB2-M F3 shows a velocity gradient, and it is possible that this arises from rotation of an accretion disk around an $\mathrm{HCH}$ II region.

\subsection{Molecular Abundances}

\subsubsection{Freeze-out}

The self-absorption features provide information on the cold foreground gas. It is however difficult to disentangle density and abundance effects. Under the assumption of spherical symmetry and a power-law density distribution, a depletion at low temperatures of $\mathrm{HCN}$ and $\mathrm{HCO}^{+}$is necessary to fit the line shapes measured with APEX. While this depletion could be due to freeze-out of $\mathrm{HCN}$ on dust grains, the reduced abundance of $\mathrm{HCO}^{+}$is more likely a secondary effect.

\subsubsection{Hot Molecules}

$\mathrm{HCN}$ is found to be very abundant in the hot gas. This result can be derived independently from each data set on the basis of vibrationally excited HCN. The abundance can reach a few times $10^{-5}$ at high temperatures $(>100-300$ $\mathrm{K})$. It is substantially enhanced with respect to lower temperatures, at least in those sources where vibrationally excited $\mathrm{H}^{13} \mathrm{CN}$ is detected with APEX. In the remaining sources the abundance could stay the same as at lower temperatures. 
Also $\mathrm{HNC}$ is enhanced at high temperatures, as the vibrationally excited $\mathrm{HN}^{13} \mathrm{C}$ in $\mathrm{SgrB} 2-\mathrm{M}$ demonstrates. $\mathrm{HC}_{3} \mathrm{~N}$ has a high abundance in the hot gas, too. All these molecular abundances are probably related by a gas-phase chemistry network that produces these molecules at high temperatures. A key could be water, whose abundance probably surpasses that of $\mathrm{CO}$ at high temperatures and which is about to be studied in detail with Herschel (e.g. van Dishoeck et al. 2011). As its high-temperature production consumes $\mathrm{OH}$, it could allow a rich $\mathrm{N}$-chemistry (Rodgers \& Charnley 2001).

\subsection{Implications for High-Mass Star Formation}

\subsubsection{Large Mass of Hot Gas}

The mass of hot gas found in many HMCs is much larger than previously thought, namely on the order of hundreds of solar masses which have temperatures in excess of $300 \mathrm{~K}$. This has profound impacts on the formation of star clusters.

Diffusion of radiation is needed to increase the temperature in the central region. This implies large column densities in all directions, so highly filamentary, clumpy, or disk-like structures have at least to be embedded in more homogeneous, optically thick envelopes that further reprocess most of their dust emission.

The high density in molecular gas in the whole central region at a time when massive stars have already formed poses the question of where the low-mass stars are. If they have formed simultaneously to the high-mass stars, they could still be at an early evolutionary stage, having their own accretion disk or small core, and not be affected much by the surrounding gas. They are very difficult to detect due to the large distances. On the other hand, if low-mass stars have not yet formed in the central region, they cannot anymore due to the high pressure. In this case, they form only farther outside, and the central IMF is very top-heavy.

The average gas densities in the HMCs are on the order of $10^{5}$ to $10^{6} \mathrm{M}_{\odot} \mathrm{pc}^{-3}$, depending on where the radius is set. This is more than the stellar density in young massive star clusters. Further star formation could transfer part of this gas mass into star mass, or the density could be reduced by expansion and mass loss. A connection to later stages of star cluster evolution is thus possible, e.g. through the missing embedded starburst clusters (Pfalzner 2009).

\subsubsection{Onset of Feedback}

The formation of massive stars produces a variety of feedback onto the surrounding gas. Consequences are beginning ionization, traced by the HCHII regions, as well as a reversal of the gravitational infall, caused by the increased pressure due to stellar radiation, winds, outflows, and heating. The expansion motions can be well seen in the changing asymmetries of the HCN lines and the blue-shifted absorption in the interferometer data, while a global infall manifests itself in the red asymmetries.

The onset of feedback is thus traced by the velocity field. An important aspect is that the expansion motions are not well collimated, as is the case for outflows from low-mass stars, but have a wide impact angle. They are therefore probably not primarily caused by a single bipolar, magneto-centrifugal outflow, but rather by radiation/wind pressure aided by thermal pressure.

\subsubsection{Distribution of Matter}

This increased pressure in the inner part also determines the density distribution, and indeed a central flattening is found. A power-law density that continues in the central region is probably prevented or disrupted by feedback processes, and can be excluded by the interferometer observations.

The stars with strong ionizing radiation are well localized by the VLA data, and form multiple systems or clusters. In contrast, the dust does not show clear multiplicity in G10.47+0.03 and SgrB2-N. The apparent lack of fragmentation could be due to clumpiness on smaller scales than traced by the beam or an evolutionary sequence with first the formation of high-mass stars and then fragmentation and low-mass star formation. 


\section{Chapter 9}

\section{Conclusions}

For this $\mathrm{PhD}$ thesis, I obtained high-quality data and analyzed them with detailed radiative transfer modeling. The results improve our understanding of Hot Molecular Cores and their internal structure.

The observational data are cutting-edge both in terms of high frequencies and angular resolution. The APEX telescope, situated at an extraordinary site for submillimeter observations, allowed to advance into frequency regimes hardly accessible from the ground, and to observe spectrally resolved lines of $\mathrm{HCN}, \mathrm{HCO}^{+}, \mathrm{CO}$, and their isotopologues from a variety of energy levels, tracing different excitation conditions and optical depths. With the launch of the Herschel space observatory and the start of the HEXOS key program, this kind of study could be substantially expanded by including many more lines toward SgrB2-M, which lie at high frequencies completely absorbed by the Earth's atmosphere.

Through the use of interferometry, high angular resolution could be achieved. With both the VLA and the SMA, I obtained the highest resolution possible with these instruments. Maps of vibrationally excited HCN and free-free continuum emission were taken with the VLA in three sources, and hundreds of molecular lines and the dust continuum emission were mapped with the SMA in G10.47+0.03. Moreover, the SMA succeeded in combining high frequencies and spatial resolution by the challenging observations at $690 \mathrm{GHz}$.

To extract the information contained in the data, radiative transfer modeling is essential. I computed the temperature in a centrally heated sphere with power-law density distribution in an approximate way using diffusion of radiation in the optically thick inner part and balance between heating and cooling in the outer part. With the Monte-Carlo code RATRAN, level populations and the emitted line shapes were computed. This spherical modeling was applied to the single-dish data, which do not have sufficient angular resolution to constrain more complex models.

As a proper modeling of the interferometer data demands multiple heating sources and a non-spherical distribution of ionized and molecular gas, the three-dimensional dust and line radiative transfer code RADMC-3D was used. The dust temperature is computed from stellar heating and the molecular lines by assuming the level populations to be in Local Thermodynamic Equilibrium (LTE). It was applied to the dust emission and the lines from vibrationally excited HCN, since this is very closely in LTE with the dust temperature.

For modeling the APEX data, I developed a fitting strategy to systematically search the parameter space. It is based on exploring different molecular abundances for physical structures which fit the dust continuum. For the other data sets, I used a trial-and-error technique to reproduce the data. The unlimited diversity of possible three-dimensional structures makes an unbiased search much more difficult than for spherical models with just a handful of parameters. Models with few parameters are more easy to handle, but too simple to account for the complexity of the real sources.

The results I obtained from observations and modeling include the following. The prevalence, but not exclusivity of self-absorbed, blue asymmetric profiles of highly optically thick rotational lines imply a dominance of moderate infall. The lack of self-absorption and emission in the outer pixels of CHAMP+ must be due to clumpiness. Red asymmetric profiles point to expansion motions. A nice example of a starting reversal of infall in SgrB2-M could be detected by modeling the line asymmetries observed with Herschel/HIFI. Another example of the simultaneous presence of infall and expansion was found in the blue-shifted absorption features in G10.47+0.03, which are seen with the interferometers in addition to the infall profiles.

A density structure that follows a radial power law fits to the single-dish data, but not to the spatially resolved interferometer data. On these smaller scales, the density seems to match more closely a Plummer profile, i.e. relatively flat in the central region and rapidly falling off outwards. Clear large-scale fragmentation is missing in G10.47+0.03 and SgrB2-N, which are among the strongest line emitters, but small-scale density fluctuations are indicated by the line shapes. Heating is internal, and probably mainly by the stars that also ionize the hypercompact HII regions. With much of the current work relying on the HCN molecule and its vibrational excitation, it is now 
clear that its abundance increases with temperature, reaching high values on the order of $10^{-5}$ in the hot gas.

The combination of all the results obtained in this $\mathrm{PhD}$ thesis suggests a consistent picture of Hot Molecular Cores that is characterized by the beginning feedback from high-mass star formation. The envelope is still infalling, and the core is heated by newly formed massive stars through diffusion of radiation in a high-column-density environment. These stars also start an ionization, which is still confined to small regions, and an expansion of the gas by means of increased radiative, thermal, turbulent, and wind-driven pressure. The feedback processes are reflected in the velocity field with central expansion motions, in hypercompact HII regions, in the centrally flat density distribution, and the high temperatures. A complex chemistry develops, and the high HCN abundance found here underlines the importance of gas-phase reaction networks after the evaporation of ice mantles.

This thesis demonstrates the power of radiative transfer modeling for analyzing observational data and constraining the source structure. This method provides not just guesses, but makes it possible to exclude models in a systematic way, both by trial-and-error techniques and by scanning of a parameter space. I emphasize that the basis of progress in astronomy is good observational data, provided by state-of-the-art telescopes, such as the data that I have obtained and presented here. 


\section{Chapter 10}

\section{Outlook}

In this chapter I give a brief outlook to future possibilities and ideas in observations and data analysis. The field of observational molecular astrophysics develops fast and is on the verge of an exciting new era that is coming with ALMA. Meanwhile, theory and analysis tools profit from rising computer power.

\subsection{Observations}

Certainly the most important and ground-breaking upcoming instrument is ALMA (Atacama Large Millimeter Array), an interferometer at the APEX site that will be completed in 2013. It will consist of 50 12-m antennas that can be arranged with maximum baselines of $16 \mathrm{~km}$, plus the Atacama Compact Array (ACA) with twelve 7-m antennas and four 12-m antennas. The spectral range accessible will be 84-950 GHz. Early science, using 16 antennas and four receiver bands, will start in September 2011, for which the first call for proposals was issued recently (30 March 2011).

ALMA will allow to image Hot Molecular Cores at $0.01^{\prime \prime}$ resolution in the dust continuum and a large variety of molecular lines at once. Although at such high resolution the signal-to-noise ratio will be marginal for the lines ( $\sim 100 \mathrm{~K}$ noise in $1 \mathrm{~km} \mathrm{~s}^{-1}$ channels, as in my VLA data), a slightly lower resolution would increase the sensitivity sufficiently. The good uv coverage will guarantee the sensitivity to extended emission as well. Such observations will reveal unprecedented details about the structure of HMCs, thereby significantly improving our understanding of (clustered) high-mass star formation and astrochemistry.

Similar data cubes, although with much lower resolution and sensitivity and much poorer uv coverage, already come from the SMA. The broad bandwidth ( $8 \mathrm{GHz}$ simultaneously) is comparable to the ALMA capabilities. Millimeter interferometers that have less bandwidth, but better sensitivity, are CARMA (Combined Array for Research in Millimeter Astronomy) and the IRAM Plateau de Bure interferometer. CARMA data of HCN 3-2 (including the vibrational satellite) and SiO toward W51d and G34.26 await reduction (proposal PI T. G. Phillips), and could clarify the issues of external heating and clumpiness in those sources.

The VLA currently undergoes a digital retrofit and is being equipped with new receivers (eVLA), covering 1.2 to $50 \mathrm{GHz}$ and allowing a large bandwidth and good spectral resolution. The high sensitivity will make observations of the dust continuum at $7 \mathrm{~mm}$ with high angular resolution feasible, as well as improved observations of lines, e.g. from different vibrational levels of $\mathrm{HC}_{3} \mathrm{~N}$.

The Herschel data will improve the spectral energy distributions of HMCs, constrain the line-of-sight structure through the high-frequency rotational lines, and provide important inputs to astrochemistry. All data for the HEXOS project are now taken. In addition, a proposal to observe HCN lines in a few HMCs is accepted (PI P. Schilke). Another important field for Herschel will be the study of water in HMCs, complemented by a recently accepted APEX proposal (PI F. Wyrowski) to observe $\mathrm{H}_{2}^{18} \mathrm{O}$, based on my APEX data of the $692 \mathrm{GHz}$ line. SOFIA (Stratospheric Observatory for Infrared Astronomy) is a $2.5-\mathrm{m}$ airplane telescope that is just starting to observe at $\mathrm{THz}$ frequencies.

\subsection{Analysis}

Just as important as the data acquirement is their analysis. The obvious next step is three-dimensional radiative transfer modeling in non-LTE, i.e. exactly computing the level populations. This will allow to apply the models also to ground-state lines, which are not in LTE. Possible radiative transfer codes for this are an expanded version of RADMC-3D (which is planned to include exact non-LTE line transfer) and LIME (Brinch \& Hogerheijde 2010). The latter code uses flexible unstructured grids, but needs also the temperature as an input. 
Typical data which demand this kind of radiative transfer modeling are the spectral line data cubes from the high-resolution SMA observations of SgrB2-N and -M. Many lines from different energy levels and molecules with spectrally resolved line shapes are seen in maps of high dynamic range. These properties are also characteristic of future ALMA data.

The question arises how to find a fit to the data. For three-dimensional models, a large limitation in possible models has to be made by defining the parameter set. The problem of finding the optimal combination of parameters and estimating their errors could be solved with the help of automatic algorithms that scan the parameter space, such as those implemented in the fitting tool MAGIX (see also Sect. 2.6.6). However, such methods require the computation of many models, each taking up much computational time, and hence large computing power.

A direct comparison of observational data to hydrodynamical simulations of star formation is currently prevented by the different initial conditions of a simulation and a real source. When computational power allows the simulations of many different conditions, the outcome could be parameterized. By fitting the parameters to reproduce real data, much insight into the physics and time evolution of high-mass star formation will be gained.

Astrochemical calculations of molecular abundances could be coupled to such simulations. In this case, the chemical evolution would be followed in parallel to the changes of the physical structure. More feasible would probably be the detailed computation of the chemistry for a limited number of changing physical conditions, their parameterization, and interpolation to determine the abundances in an approximate way for the whole simulation.

Computationally much less intense, and hence easily applicable to a wide range of initial conditions, would be an analytical approach to the emerging of structures. A simplified physical modeling of pressure due to gravity and feedback could lead to density distributions and velocity fields, taking e.g. the momentum of an initial collapse into account. Such computations could result in similar structures as those modeled in this thesis, e.g. Plummerlike density distributions and expansion starting in the inner part. However, the computations might become very complex for non-spherical geometries, and gradually cross over to the hydrodynamical simulations. 


\section{Acknowledgments}

My foremost thanks go to Peter Schilke, who was the thesis advisor and accompanied my research continuously through discussions, advice, and questions. His ideas always helped to find solutions, and it is on a large part his merit that my thesis project went so successfully.

I am grateful to Karl Menten for being a referee of this thesis and for providing the pleasant and stimulating workplace at the MPIfR. Also my colleagues at the submm group contributed to the friendly and creative research atmosphere.

I am thankful to the International Max Planck Research School (IMPRS) for Astronomy and Astrophysics for the financial and organisational frame of this thesis, and for bringing together the PhD students.

Many thanks go to the co-authors of my papers for helping to obtain the data and to improve the manuscripts, and to Friedrich Wyrowski for being a member of my thesis committee and his manifold help. I appreciate that Claus Kiefer and Volker Ossenkopf have joined my PhD examination committee.

I am grateful to my parents for having inspired my curiosity and for the support of my studies of physics and astronomy.

I especially thank my wife Jasmin for her patient help and the great free-time that we share, and my daughter Clara for the funny moments and the deep happiness she evokes. 


\section{Bibliography}

Andre, P., Ward-Thompson, D., \& Barsony, M., Submillimeter continuum observations of Rho Ophiuchi A - The candidate protostar VLA 1623 and prestellar clumps. 1993, ApJ, 406, 122

Araya, E., Hofner, P., Kurtz, S., Bronfman, L., \& DeDeo, S., $\mathrm{CH}_{3} \mathrm{CN}$ Observations toward Southern Massive Star-forming Regions. 2005, ApJS, 157, 279

Araya, E., Hofner, P., Kurtz, S., Olmi, L., \& Linz, H., Thermal Methanol Observations of the Outflow from the G31.41+0.31 Hot Molecular Core. 2008, ApJ, 675, 420

Ballesteros-Paredes, J., Six myths on the virial theorem for interstellar clouds. 2006, MNRAS, 372, 443

Ballesteros-Paredes, J., Hartmann, L. W., Vázquez-Semadeni, E., Heitsch, F., \& Zamora-Avilés, M. A., Gravity or turbulence? Velocity dispersion-size relation. 2011, MNRAS, 411, 65

Ballesteros-Paredes, J., Vázquez-Semadeni, E., \& Scalo, J., Clouds as Turbulent Density Fluctuations: Implications for Pressure Confinement and Spectral Line Data Interpretation. 1999, ApJ, 515, 286

Bally, J. \& Zinnecker, H., The Birth of High-Mass Stars: Accretion and/or Mergers? 2005, AJ, 129, 2281

Barber, R. J., Harris, G. J., \& Tennyson, J., Temperature dependent partition functions and equilibrium constant for HCN and HNC. 2002, J. Chem. Phys., 117, 24

Belloche, A., Comito, C., Hieret, C., et al., The search for complex molecules in the ISM: a complete $3 \mathrm{~mm}$ line survey of Sgr B2-N and -M. 2007, in Molecules in Space and Laboratory

Belloche, A., Menten, K. M., Comito, C., et al., Detection of amino acetonitrile in Sgr B2(N). 2008, A\&A, 482, 179

Beltrán, M. T., Cesaroni, R., Neri, R., \& Codella, C., Rotating toroids in G10.62-0.38, G19.61-0.23, and G29.960.02. 2011, A\&A, 525, A151+

Beltrán, M. T., Cesaroni, R., Neri, R., et al., A detailed study of the rotating toroids in G31.41+0.31 and G24.78+0.08. 2005, A\&A, 435, 901

Bergin, E. A., Phillips, T. G., Comito, C., et al., Herschel observations of EXtra-Ordinary Sources (HEXOS): The present and future of spectral surveys with Herschel/HIFI. 2010, A\&A, 521, L20+

Bernes, C., A Monte Carlo approach to non-LTE radiative transfer problems. 1979, A\&A, 73, 67

Beuther, H., Physics and chemistry of hot molecular cores. 2007, in IAU Symposium, Vol. 237, IAU Symposium, ed. B. G. Elmegreen \& J. Palous, 148-154

Beuther, H., Schilke, P., Sridharan, T. K., et al., Massive molecular outflows. 2002, A\&A, 383, 892

Beuther, H., Walsh, A. J., \& Longmore, S. N., Hot High-Mass Accretion Disk Candidates. 2009, ApJS, 184, 366

Beuther, H., Walsh, A. J., Thorwirth, S., et al., ATCA 3 mm observations of NGC 6334I and I(N): dense cores, outflows, and an UCHII region. 2008, A\&A, 481, 169

Beuther, H., Zhang, Q., Reid, M. J., et al., Submillimeter Array $440 \mu \mathrm{m} / 690 \mathrm{GHz}$ Line and Continuum Observations of Orion KL. 2006, ApJ, 636, 323

Bonnell, I. A., Bate, M. R., Clarke, C. J., \& Pringle, J. E., Competitive accretion in embedded stellar clusters. 2001, MNRAS, 323, 785 
Bonnell, I. A., Bate, M. R., \& Zinnecker, H., On the formation of massive stars. 1998, MNRAS, 298, 93

Bonnell, I. A., Vine, S. G., \& Bate, M. R., Massive star formation: nurture, not nature. 2004, MNRAS, 349,735

Boonman, A. M. S., Stark, R., van der Tak, F. F. S., et al., Highly Abundant HCN in the Inner Hot Envelope of GL 2591: Probing the Birth of a Hot Core? 2001, ApJ, 553, L63

Brinch, C. \& Hogerheijde, M. R., LIME - a flexible, non-LTE line excitation and radiation transfer method for millimeter and far-infrared wavelengths. 2010, A\&A, 523, A25+

Brogan, C. L., Hunter, T. R., Indebetouw, R., et al., Properties of the Massive Protocluster NGC6334I. 2009, in Bulletin of the American Astronomical Society, Vol. 41, Bulletin of the American Astronomical Society, 499-+

Cameron, A. G. W., Abundances of the Elements in the Solar System. 1973, Space Science Reviews, 15, 121

Carlstrom, J. E. \& Vogel, S. N., Millimeter continuum observations of a massive protostellar core in Sagittarius B2. 1989, ApJ, 337, 408

Carroll, T. J. \& Goldsmith, P. F., Infrared pumping and rotational excitation of molecules in interstellar clouds. 1981, ApJ, 245, 891

Ceccarelli, C., Bacmann, A., Boogert, A., et al., Herschel spectral surveys of star-forming regions. Overview of the 555-636 GHz range. 2010, A\&A, 521, L22+

Cesaroni, R., Hot molecular cores. 2005, in IAU Symposium, Vol. 227, Massive Star Birth: A Crossroads of Astrophysics, ed. R. Cesaroni, M. Felli, E. Churchwell, \& M. Walmsley, 59-69

Cesaroni, R., Churchwell, E., Hofner, P., Walmsley, C. M., \& Kurtz, S., Hot ammonia towards compact HiI regions. 1994, A\&A, 288, 903

Cesaroni, R., Galli, D., Lodato, G., Walmsley, C. M., \& Zhang, Q., Disks Around Young O-B (Proto)Stars: Observations and Theory. 2007, Protostars and Planets V, 197

Cesaroni, R., Hofner, P., Araya, E., \& Kurtz, S., The structure of hot molecular cores over 1000 AU. 2010, A\&A, $509, \mathrm{~A} 50+$

Cesaroni, R., Hofner, P., Walmsley, C. M., \& Churchwell, E., Sub-arcsecond structure of hot cores in the $\mathrm{NH}_{3}$ $(4,4)$ line. 1998, A\&A, 331, 709

Churchwell, E., The Formation and Early Evolution of Massive Stars. 2002, in Astronomical Society of the Pacific Conference Series, Vol. 267, Hot Star Workshop III: The Earliest Phases of Massive Star Birth, ed. P. Crowther, $3-+$

Churchwell, E., Walmsley, C. M., \& Cesaroni, R., A survey of ammonia and water vapor emission from ultracompact Hir regions. 1990, A\&AS, 83, 119

Comito, C. \& Schilke, P., Reconstructing reality: Strategies for sideband deconvolution. 2002, A\&A, 395, 357

Comito, C., Schilke, P., Gerin, M., et al., The line-of-sight distribution of water in the SgrB2 complex. 2003, A\&A, 402,635

de Graauw, T., Helmich, F. P., Phillips, T. G., et al., The Herschel-Heterodyne Instrument for the Far-Infrared (HIFI). 2010, A\&A, 518, L6+

de Pree, C. G., Goss, W. M., \& Gaume, R. A., Ionized Gas in Sagittarius B2 Main on Scales of 0.065 Arcsecond (600 AU). 1998, ApJ, 500, 847

de Vicente, P., Martín-Pintado, J., Neri, R., \& Colom, P., A ridge of recent massive star formation between Sgr B2M and Sgr B2N. 2000, A\&A, 361, 1058

Dedes, C., Leurini, S., Wyrowski, F., et al., A study of three southern high-mass star-forming regions. 2011, A\&A, 526, A59+

Deharveng, L. \& Zavagno, A., Massive Star Formation Triggered by Galactic HII Regions. 2008, in Astronomical Society of the Pacific Conference Series, Vol. 387, Massive Star Formation: Observations Confront Theory, ed. H. Beuther, H. Linz, \& T. Henning, 338-+ 
Duley, W. W. \& Williams, D. A., The formation of $\mathrm{H}_{2}$ on interstellar dust. 1993, MNRAS, 260, 37

Dumouchel, F., Faure, A., \& Lique, F., The rotational excitation of HCN and HNC by He: temperature dependence of the collisional rate coefficients. 2010, MNRAS, 406, 2488

Einstein, A., Strahlungs-Emission und -Absorption nach der Quantentheorie. 1916, Deutsche Physikalische Gesellschaft, 18, 318

Elitzur, M. \& Asensio Ramos, A., A new exact method for line radiative transfer. 2006, MNRAS, 365, 779

Emprechtinger, M., Lis, D. C., Bell, T., et al., The distribution of water in the high-mass star-forming region NGC 6334 I. 2010, A\&A, 521, L28+

Evans, II, N., Studying Infall. 2003, in SFChem 2002: Chemistry as a Diagnostic of Star Formation, ed. C. L. Curry \& M. Fich, 157-+

Faúndez, S., Bronfman, L., Garay, G., et al., SIMBA survey of southern high-mass star forming regions. I. Physical parameters of the $1.2 \mathrm{~mm} / \mathrm{IRAS}$ sources. 2004, A\&A, 426, 97

Flower, D. R. \& Launay, J. M., Rate coefficients for the rotational excitation of CO by ortho- and para- $\mathrm{H}_{2} .1985$, MNRAS, 214, 271

Friedel, D. N., Snyder, L. E., Turner, B. E., \& Remijan, A., A Spectral Line Survey of Selected 3 Millimeter Bands toward Sagittarius B2(N-LMH) Using the National Radio Astronomy Observatory 12 Meter Radio Telescope and the Berkeley-Illinois-Maryland Association Array. I. The Observational Data. 2004, ApJ, 600, 234

Fuchs, U., Bruenken, S., Fuchs, G. W., et al., High Resolution Spectroscopy of HCN Isotopomers: $\mathrm{H}^{13} \mathrm{CN}, \mathrm{HC}^{15} \mathrm{~N}$, and $\mathrm{H}^{13} \mathrm{C}^{15} \mathrm{~N}$ in the Ground and First Excited Bending Vibrational State. 2004, Z. Naturforsch. A, 59, 861

Furuya, R. S., Cesaroni, R., \& Shinnaga, H., Infall, outflow, and rotation in the G19.61-0.23 hot molecular core. 2011, A\&A, 525, A72+

Gallimore, J. F., Henkel, C., Baum, S. A., et al., The Nature of the Nuclear $\mathrm{H}_{2} \mathrm{O}$ Masers of NGC 1068: Reverberation and Evidence for a Rotating Disk Geometry. 2001, ApJ, 556, 694

Garrod, R. T., Weaver, S. L. W., \& Herbst, E., Complex Chemistry in Star-forming Regions: An Expanded GasGrain Warm-up Chemical Model. 2008, ApJ, 682, 283

Gaume, R. A. \& Claussen, M. J., The Sagittarius B2 star-forming region - Subarcsecond radio spectral line and continuum observations. 1990, ApJ, 351, 538

Gaume, R. A., Claussen, M. J., de Pree, C. G., Goss, W. M., \& Mehringer, D. M., The Sagittarius B2 Star-forming Region. I. Sensitive 1.3 Centimeter Continuum Observations. 1995, ApJ, 449, 663

Genzel, R., Physical Conditions and Heating/Cooling Processes in High Mass Star Formation Regions. 1991, in NATO ASIC Proc. 342: The Physics of Star Formation and Early Stellar Evolution, ed. C. J. Lada \& N. D. Kylafis, 155-+

Gibb, E., Nummelin, A., Irvine, W. M., Whittet, D. C. B., \& Bergman, P., Chemistry of the Organic-Rich Hot Core G327.3-0.6. 2000, ApJ, 545, 309

Goldsmith, P. F., Lis, D. C., Lester, D. F., \& Harvey, P. M., High angular resolution far-infrared observations of Sagittarius B2. 1992, ApJ, 389, 338

Green, S. \& Thaddeus, P., Rotational Excitation of HCN by Collisions. 1974, ApJ, 191, 653

Güsten, R., Baryshev, A., Bell, A., et al., Submillimeter heterodyne arrays for APEX. 2008, in Presented at the Society of Photo-Optical Instrumentation Engineers (SPIE) Conference, Vol. 7020, Society of Photo-Optical Instrumentation Engineers (SPIE) Conference Series

Güsten, R., Nyman, L. A., Schilke, P., et al., The Atacama Pathfinder EXperiment (APEX) - a new submillimeter facility for southern skies -. 2006, A\&A, 454, L13

Haken, H. \& Wolf, H. C., Molekülphysik und Quantenchemie. 2003 (Molekülphysik und Quantenchemie, by Hermann Haken, Hans Christoph Wolf, pp. 528. ISBN 3-540-43551-4. Springer, January 2003.)

Hatchell, J. \& van der Tak, F. F. S., The physical structure of high-mass star-forming cores. 2003, A\&A, 409, 589 
Hauschildt, H., Güsten, R., Phillips, T. G., et al., First Detection of CS/10-9/ in Galactic Star Forming Cores. 1993, A\&A, 273, L23+

Hennebelle, P. \& Fromang, S., Magnetic processes in a collapsing dense core. I. Accretion and ejection. 2008, A\&A, 477, 9

Henning, T., Lapinov, A., Schreyer, K., Stecklum, B., \& Zinchenko, I., IRAS 12326-6245: Luminous very young stellar objects with a massive molecular outflow. 2000, A\&A, 364, 613

Herbst, E. \& van Dishoeck, E. F., Complex Organic Interstellar Molecules. 2009, ARA\&A, 47, 427

Heyminck, S., Kasemann, C., Güsten, R., de Lange, G., \& Graf, U. U., The first-light APEX submillimeter heterodyne instrument FLASH. 2006, A\&A, 454, L21

Hildebrand, R. H., The Determination of Cloud Masses and Dust Characteristics from Submillimetre Thermal Emission. 1983, QJRAS, 24, 267

Ho, P. T. P., Moran, J. M., \& Lo, K. Y., The Submillimeter Array. 2004, ApJ, 616, L1

Ho, P. T. P. \& Young, L. M., The Contracting Molecular Cores e1 and e2 in W51. 1996, ApJ, 472, 742

Hoare, M. G., Kurtz, S. E., Lizano, S., Keto, E., \& Hofner, P., Ultracompact HiI Regions and the Early Lives of Massive Stars. 2007, Protostars and Planets V, 181

Hofner, P. \& Churchwell, E., A survey of water maser emission toward ultracompact HII regions. 1996, A\&AS, 120,283

Hogerheijde, M. R. \& van der Tak, F. F. S., An accelerated Monte Carlo method to solve two-dimensional radiative transfer and molecular excitation. With applications to axisymmetric models of star formation. 2000, A\&A, 362, 697

Hollenbach, D., Johnstone, D., Lizano, S., \& Shu, F., Photoevaporation of disks around massive stars and application to ultracompact HII regions. 1994, ApJ, 428, 654

Hollis, J. M., Pedelty, J. A., Boboltz, D. A., et al., Kinematics of the Sagittarius B2(N-LMH) Molecular Core. 2003, ApJ, 596, L235

Hosokawa, T. \& Omukai, K., Evolution of Massive Protostars with High Accretion Rates. 2009, ApJ, 691, 823

Huebner, W. F., Buhl, D., \& Snyder, L. E., HCN radio emission from Comet Kohoutek /1973f/. 1974, Icarus, 23, 580

Hunter, T. R., Brogan, C. L., Megeath, S. T., et al., Millimeter Multiplicity in NGC 6334 I and I(N). 2006, ApJ, 649,888

Ivezic, Z., Nenkova, M., \& Elitzur, M., User Manual for DUSTY. 1999, University of Kentucky Internal Report, accessible at http://www.pa.uky.edu/ moshe/dusty

Jones, P. A., Burton, M. G., Cunningham, M. R., et al., Spectral imaging of the Sagittarius B2 region in multiple 3-mm molecular lines with the Mopra telescope. 2008, MNRAS, 386, 117

Kasemann, C., Güsten, R., Heyminck, S., et al., CHAMP ${ }^{+}$: a powerful array receiver for APEX. 2006, in Presented at the Society of Photo-Optical Instrumentation Engineers (SPIE) Conference, Vol. 6275, Society of PhotoOptical Instrumentation Engineers (SPIE) Conference Series

Kaufman, M. J., Hollenbach, D. J., \& Tielens, A. G. G. M., High-Temperature Molecular Cores near Massive Stars and Application to the Orion Hot Core. 1998, ApJ, 497, 276

Keto, E., The Formation of Massive Stars by Accretion through Trapped Hypercompact HiI Regions. 2003, ApJ, 599,1196

Keto, E., Rybicki, G. B., Bergin, E. A., \& Plume, R., Radiative Transfer and Starless Cores. 2004, ApJ, 613, 355

Keto, E. \& Wood, K., Observations on the Formation of Massive Stars by Accretion. 2006, ApJ, 637, 850

Klein, B., Philipp, S. D., Krämer, I., et al., The APEX digital Fast Fourier Transform Spectrometer. 2006, A\&A, 454, L29 
Klessen, R. S., Ballesteros-Paredes, J., Li, Y., \& Mac Low, M., Gravoturbulent Star Cluster Formation. 2004, in Astronomical Society of the Pacific Conference Series, Vol. 322, The Formation and Evolution of Massive Young Star Clusters, ed. H. J. G. L. M. Lamers, L. J. Smith, \& A. Nota, 299-+

Kroupa, P., The Initial Mass Function of Stars: Evidence for Uniformity in Variable Systems. 2002, Science, 295, 82

Krumholz, M. R., Klein, R. I., McKee, C. F., Offner, S. S. R., \& Cunningham, A. J., The Formation of Massive Star Systems by Accretion. 2009, Science, 323, 754

Krumholz, M. R. \& Matzner, C. D., The Dynamics of Radiation-pressure-dominated Hir Regions. 2009, ApJ, 703, 1352

Krumholz, M. R. \& McKee, C. F., A minimum column density of $1 \mathrm{~g} \mathrm{~cm}^{-2}$ for massive star formation. 2008, Nature, 451, 1082

Kurtz, S., Hypercompact Hir regions. 2005, in IAU Symposium, Vol. 227, Massive Star Birth: A Crossroads of Astrophysics, ed. R. Cesaroni, M. Felli, E. Churchwell, \& M. Walmsley, 111-119

Kurtz, S., Cesaroni, R., Churchwell, E., Hofner, P., \& Walmsley, C. M., Hot Molecular Cores and the Earliest Phases of High-Mass Star Formation. 2000, Protostars and Planets IV, 299

Lada, C. J. \& Lada, E. A., Embedded Clusters in Molecular Clouds. 2003, ARA\&A, 41, 57

Larson, R. B., Numerical calculations of the dynamics of collapsing proto-star. 1969a, MNRAS, 145, 271

Larson, R. B., The emitted spectrum of a proto-star. 1969b, MNRAS, 145, 297

Leurini, S., Codella, C., Zapata, L. A., et al., Extremely high velocity gas from the massive young stellar objects in IRAS 17233-3606. 2009, A\&A, 507, 1443

Leurini, S., Hieret, C., Thorwirth, S., et al., High-mass star formation in the IRAS 17233-3606 region: a new nearby and bright hot core in the southern sky. 2008, A\&A, 485, 167

Li, Z. \& Nakamura, F., Cluster Formation in Protostellar Outflow-driven Turbulence. 2006, ApJ, 640, L187

Lis, D. C., Carlstrom, J. E., \& Keene, J., Millimeter continuum observations of Galactic center giant molecular cloud cores. 1991, ApJ, 380, 429

Lis, D. C. \& Goldsmith, P. F., Modeling of the continuum and molecular line emission from the Sagittarius B2 molecular cloud. 1990, ApJ, 356, 195

Lis, D. C., Goldsmith, P. F., Carlstrom, J. E., \& Scoville, N. Z., Millimeter-wavelength aperture synthesis observations of massive star-forming regions in Sagittarius B2. 1993, ApJ, 402, 238

Liu, S.-Y. \& Snyder, L. E., Subarcsecond Resolution Observations of Sagittarius B2 at 85 GHZ. 1999, ApJ, 523, 683

Loren, R. B. \& Mundy, L. G., The methyl cyanide hot and warm cores in Orion - Statistical equilibrium excitation models of a symmetric-top molecule. 1984, ApJ, 286, 232

Mac Low, M. \& Klessen, R. S., Control of star formation by supersonic turbulence. 2004, Reviews of Modern Physics, 76, 125

MacLeod, G. C., Scalise, E. J., Saedt, S., Galt, J. A., \& Gaylard, M. J., Masers in Massive Star-Forming Regions Associated with the Brightest Steep-Spectrum IRAS Point Sources. 1998, AJ, 116, 1897

Maki, A. G., Microwave spectra of molecules of astrophysical interest. 6. Carbonyl sulfide and hydrogen cyanide. 1974, Journal of Physical and Chemical Reference Data, 3, 221

Maki, A. G., Mellau, G. C., Klee, S., Winnewisser, M., \& Quapp, W., High-Temperature Infrared Measurements in the Region of the Bending Fundamental of $\mathrm{H}^{12} \mathrm{C}^{14} \mathrm{~N}, \mathrm{H}^{12} \mathrm{C}^{15} \mathrm{~N}$, and $\mathrm{H}^{13} \mathrm{C}^{14} \mathrm{~N}$. 2000, Journal of Molecular Spectroscopy, 202, 67

Masunaga, H., Miyama, S. M., \& Inutsuka, S., A Radiation Hydrodynamic Model for Protostellar Collapse. I. The First Collapse. 1998, ApJ, 495, 346 
Mathis, J. S., Rumpl, W., \& Nordsieck, K. H., The size distribution of interstellar grains. 1977, ApJ, 217, 425

McKee, C. F. \& Ostriker, J. P., A theory of the interstellar medium - Three components regulated by supernova explosions in an inhomogeneous substrate. 1977, ApJ, 218, 148

McKee, C. F. \& Tan, J. C., The Formation of Massive Stars from Turbulent Cores. 2003, ApJ, 585, 850

Mehringer, D. M. \& Menten, K. M., 44 GHz Methanol Masers and Quasi-thermal Emission in Sagittarius B2. 1997, ApJ, 474, 346

Menten, K. M., Pillai, T., \& Wyrowski, F., Initial conditions for massive star birth-Infrared dark clouds. 2005, in IAU Symposium, Vol. 227, Massive Star Birth: A Crossroads of Astrophysics, ed. R. Cesaroni, M. Felli, E. Churchwell, \& M. Walmsley, 23-34

Menten, K. M., Reid, M. J., Forbrich, J., \& Brunthaler, A., The distance to the Orion Nebula. 2007, A\&A, 474, 515

Minier, V., André, P., Bergman, P., et al., Evidence of triggered star formation in G327.3-0.6. Dust-continuum mapping of an infrared dark cloud with P-ArTéMiS. 2009, A\&A, 501, L1

Mookerjea, B., Casper, E., Mundy, L. G., \& Looney, L. W., Kinematics and Chemistry of the Hot Molecular Core in G34.26+0.15 at High Resolution. 2007, ApJ, 659, 447

Morris, M., Palmer, P., \& Zuckerman, B., Hot ammonia in Orion. 1980, ApJ, 237, 1

Müller, H. S. P., Schlöder, F., Stutzki, J., \& Winnewisser, G., The Cologne Database for Molecular Spectroscopy, CDMS: a useful tool for astronomers and spectroscopists. 2005, J. Mol. Struct., 742, 215

Müller, H. S. P., Thorwirth, S., Roth, D. A., \& Winnewisser, G., The Cologne Database for Molecular Spectroscopy, CDMS. 2001, A\&A, 370, L49

Myers, P. C., Filamentary Structure of Star-forming Complexes. 2009, ApJ, 700, 1609

Neckel, T., UBV, VRI and H-beta observations of stars in the HII regions NGC 6334 and NGC 6357. 1978, A\&A, 69,51

Nomura, H. \& Millar, T. J., The physical and chemical structure of hot molecular cores. 2004, A\&A, 414, 409

Nummelin, A., Bergman, P., Hjalmarson, A., et al., A Three-Position Spectral Line Survey of Sagittarius B2 between 218 and 263 GHz. I. The Observational Data. 1998, ApJS, 117, 427

Offer, A. R. \& van Dishoeck, E. F., Rotational excitation of interstellar OH by para- and ortho-H 2 . 1992, MNRAS, 257,377

Olmi, L., Cesaroni, R., Neri, R., \& Walmsley, C. M., High resolution $\mathrm{CH}_{3} \mathrm{CN}$ observations towards hot cores. 1996, A\&A, 315, 565

Osorio, M., Anglada, G., Lizano, S., \& D’Alessio, P., Collapsing Hot Molecular Cores: A Model for the Dust Spectrum and Ammonia Line Emission of the G31.41+0.31 Hot Core. 2009, ApJ, 694, 29

Osorio, M., Lizano, S., \& D’Alessio, P., Hot Molecular Cores and the Formation of Massive Stars. 1999, ApJ, 525, 808

Ossenkopf, V. \& Henning, T., Dust opacities for protostellar cores. 1994, A\&A, 291, 943

Osterloh, M., Henning, T., \& Launhardt, R., Infrared Images and Millimeter Data from Cold Southern IRAS Sources. 1997, ApJS, 110, 71

Ott, M., Witzel, A., Quirrenbach, A., et al., An updated list of radio flux density calibrators. 1994, A\&A, 284, 331

Ott, S., The Herschel Data Processing System - HIPE and Pipelines - Up and Running Since the Start of the Mission. 2010, in Astronomical Society of the Pacific Conference Series, Vol. 434, Astronomical Data Analysis Software and Systems XIX, ed. Y. Mizumoto, K.-I. Morita, \& M. Ohishi, 139-+

Panagia, N., Some Physical parameters of early-type stars. 1973, AJ, 78, 929

Pandian, J. D., Momjian, E., \& Goldsmith, P. F., Resolving distance ambiguities towards 6.7 GHz methanol masers. 2008, A\&A, 486, 191 
Pavlyuchenkov, Y., Semenov, D., Henning, T., et al., Molecular Line Radiative Transfer in Protoplanetary Disks: Monte Carlo Simulations versus Approximate Methods. 2007, ApJ, 669, 1262

Penston, M. V., Dynamics of self-gravitating gaseous spheres-III. Analytical results in the free-fall of isothermal cases. 1969, MNRAS, 144, 425

Peters, T., Banerjee, R., Klessen, R. S., \& Mac Low, M., The Interplay of Magnetic Fields, Fragmentation, and Ionization Feedback in High-mass Star Formation. 2011, ApJ, 729, 72

Peters, T., Banerjee, R., Klessen, R. S., et al., Hir Regions: Witnesses to Massive Star Formation. 2010, ApJ, 711, 1017

Pfalzner, S., Universality of young cluster sequences. 2009, A\&A, 498, L37

Pilbratt, G. L., Riedinger, J. R., Passvogel, T., et al., Herschel Space Observatory. An ESA facility for far-infrared and submillimetre astronomy. 2010, A\&A, 518, L1+

Qin, S., Zhao, J., Moran, J. M., et al., Infall and Outflow of Molecular Gas in Sgr B2. 2008, ApJ, 677, 353

Qin, S. L., Schilke, P., Rolffs, R., et al., Submillimeter Continuum Observations of Sagittarius B2 at Subarcsecond Spatial Resolution. 2011, A\&A, submitted

Qiu, K., Zhang, Q., \& Menten, K. M., Outflows, Accretion, and Clustered Protostellar Cores Around a Forming O Star. 2011, ApJ, 728, 6

Rathborne, J. M., Jackson, J. M., \& Simon, R., Infrared Dark Clouds: Precursors to Star Clusters. 2006, ApJ, 641, 389

Reid, M. J., Menten, K. M., Zheng, X. W., Brunthaler, A., \& Xu, Y., A Trigonometric Parallax of Sgr B2. 2009, ApJ, 705, 1548

Risacher, C., Vassilev, V., Monje, R., et al., A $0.8 \mathrm{~mm}$ heterodyne facility receiver for the APEX telescope. 2006, A\&A, 454, L17

Rodgers, S. D. \& Charnley, S. B., Chemical Differentiation in Regions of Massive Star Formation. 2001, ApJ, 546, 324

Rohlfs, K. \& Wilson, T. L., Tools of radio astronomy. 2004 (Tools of radio astronomy, 4th rev. and enl. ed., by K. Rohlfs and T.L. Wilson. Berlin: Springer, 2004)

Rolffs, R., Constraining the structure of hot cores through exact radiative transfer modeling of molecular lines. 2007, Diploma Thesis, Universität Bonn

Rolffs, R., Schilke, P., Comito, C., et al., Reversal of infall in SgrB2(M) revealed by Herschel/HIFI observations of HCN lines at THz frequencies. 2010, A\&A, 521, L46+

Rolffs, R., Schilke, P., Wyrowski, F., et al., Hot HCN around young massive stars at 0.1" resolution. 2011a, A\&A, 529, A76+

Rolffs, R., Schilke, P., Wyrowski, F., et al., Structure of evolved cluster-forming regions. 2011b, A\&A, 527, A68+

Rolffs, R., Schilke, P., Zhang, Q., \& Zapata, L., Structure of the Hot Molecular Core G10.47+0.03. 2011c, A\&A, submitted

Rudolph, A., Welch, W. J., Palmer, P., \& Dubrulle, B., Dynamical collapse of the W51 star-forming region. 1990, ApJ, 363, 528

Rybicki, G. B. \& Hummer, D. G., An accelerated lambda iteration method for multilevel radiative transfer. I Non-overlapping lines with background continuum. 1991, A\&A, 245, 171

Rybicki, G. B. \& Lightman, A. P., Radiative processes in astrophysics. 1979 (New York, Wiley-Interscience, 1979. 393 p.)

Salter, C. J., Ghosh, T., Catinella, B., et al., The Arecibo ARP 220 Spectral Census. I. Discovery of the Pre-Biotic Molecule Methanimine and New Cm-Wavelength Transitions of Other Molecules. 2008, AJ, 136, 389

Sandell, G., (Sub)mm continuum mapping of NGC 6334 I \& I(N). A cobweb of filaments and protostars. 2000, A\&A, 358, 242 
Sato, M., Reid, M. J., Brunthaler, A., \& Menten, K. M., Trigonometric Parallax of W51 Main/South. 2010, ApJ, 720,1055

Sault, R. J., Teuben, P. J., \& Wright, M. C. H., A Retrospective View of MIRIAD. 1995, in Astronomical Society of the Pacific Conference Series, Vol. 77, Astronomical Data Analysis Software and Systems IV, ed. R. A. Shaw, H. E. Payne, \& J. J. E. Hayes, 433-+

Schilke, P., Comito, C., \& Thorwirth, S., First Detection of Vibrationally Excited HNC in Space. 2003, ApJ, 582, L101

Schilke, P., Comito, C., Thorwirth, S., et al., Submillimeter spectroscopy of southern hot cores: NGC 6334(I) and G327.3-0.6. 2006, A\&A, 454, L41

Schilke, P., Mehringer, D. M., \& Menten, K. M., A Submillimeter HCN Laser in IRC +10216. 2000, ApJ, 528, L37

Schilke, P., Walmsley, C. M., Pineau Des Forets, G., et al., A study of HCN, HNC and their isotopomers in OMC-1. I - Abundances and chemistry. 1992, A\&A, 256, 595

Schöier, F. L., van der Tak, F. F. S., van Dishoeck, E. F., \& Black, J. H., An atomic and molecular database for analysis of submillimetre line observations. 2005, A\&A, 432, 369

Schuller, F., Menten, K. M., Contreras, Y., et al., ATLASGAL - The APEX telescope large area survey of the galaxy at $870 \mu \mathrm{m} .2009, \mathrm{~A} \& \mathrm{~A}, 504,415$

Shepherd, D., Massive star outflows. 2005, in IAU Symposium, Vol. 227, Massive Star Birth: A Crossroads of Astrophysics, ed. R. Cesaroni, M. Felli, E. Churchwell, \& M. Walmsley, 237-246

Shetty, R., Glover, S. C., Dullemond, C. P., \& Klessen, R. S., Modelling CO emission - I. CO as a column density tracer and the $\mathrm{X}$ factor in molecular clouds. 2011, MNRAS, 11

Shi, H., Zhao, J., \& Han, J. L., Nature of W51e2: Massive Cores at Different Phases of Star Formation. 2010, ApJ, 710,843

Shu, F. H., Self-similar collapse of isothermal spheres and star formation. 1977, ApJ, 214, 488

Simpson, J. P. \& Rubin, R. H., IRAS low-resolution spectral observations of HII regions. 1990, ApJ, 354, 165

Siringo, G., Kreysa, E., Kovács, A., et al., The Large APEX BOlometer CAmera LABOCA. 2009, A\&A, 497, 945

Snyder, L. E., Kuan, Y., \& Miao, Y., Where Is the Heavy Molecule Heimat in SgrB2? 1994, in Lecture Notes in Physics, Berlin Springer Verlag, Vol. 439, The Structure and Content of Molecular Clouds, ed. T. L. Wilson \& K. J. Johnston, 187-+

Sobolev, V. V., Moving envelopes of stars. 1960 (Cambridge: Harvard University Press, 1960)

Spitzer, L., Physical processes in the interstellar medium. 1978 (New York Wiley-Interscience, 1978. 333 p.)

Sridharan, T. K., Beuther, H., Schilke, P., Menten, K. M., \& Wyrowski, F., High-Mass Protostellar Candidates. I. The Sample and Initial Results. 2002, ApJ, 566, 931

Stahler, S. W. \& Palla, F., The Formation of Stars. 2005 (The Formation of Stars, by Steven W. Stahler, Francesco Palla, pp. 865. ISBN 3-527-40559-3. Wiley-VCH , January 2005.)

Stutzki, J., Genzel, R., Harris, A. I., Herman, J., \& Jaffe, D. T., First detection of HCN J = 9-8 (797 GHz) line emission - Very high densities in the Orion core. 1988, ApJ, 330, L125

Thorwirth, S., Carbon Chain- and Ring-Molecules in the Laboratory and in Space. 2001, PhD thesis, Universität zu Köln

Thorwirth, S., Müller, H. S. P., Lewen, F., et al., A Concise New Look at the 1-Type Spectrum of $\mathrm{H}^{12} \mathrm{C}^{14}$ N. 2003a, ApJ, 585, L163

Thorwirth, S., Walsh, A. J., Wyrowski, F., et al., Millimeter wave spectral line surveys and line mapping studies of NGC6334 I and I(N). 2007, in Molecules in Space and Laboratory

Thorwirth, S., Wyrowski, F., Schilke, P., et al., Detection of HCN Direct 1-Type Transitions Probing Hot Molecular Gas in the Proto-Planetary Nebula CRL 618. 2003b, ApJ, 586, 338 
Tielens, A. G. G. M., The Physics and Chemistry of the Interstellar Medium. 2005 (The Physics and Chemistry of the Interstellar Medium, by A. G. G. M. Tielens, pp. . ISBN 0521826349. Cambridge, UK: Cambridge University Press, 2005.)

van der Tak, F. F. S., Observations and Models of The Embedded Phase of High-Mass Star Formation. 2002, in Astronomical Society of the Pacific Conference Series, Vol. 267, Hot Star Workshop III: The Earliest Phases of Massive Star Birth, ed. P. Crowther, 33-+

van der Tak, F. F. S., Hot Molecular Cores and High Mass Star Formation. 2004, in IAU Symposium, Vol. 221, Star Formation at High Angular Resolution, ed. M. Burton, R. Jayawardhana, \& T. Bourke, 59-+

van der Tak, F. F. S. \& van Dishoeck, E. F., Limits on the cosmic-ray ionization rate toward massive young stars. 2000, A\&A, 358, L79

van Dishoeck, E. F., Kristensen, L. E., Benz, A. O., et al., Water in Star-forming Regions with the Herschel Space Observatory (WISH). I. Overview of Key Program and First Results. 2011, PASP, 123, 138

van Zadelhoff, G.-J., Dullemond, C. P., van der Tak, F. F. S., et al., Numerical methods for non-LTE line radiative transfer: Performance and convergence characteristics. 2002, A\&A, 395, 373

Vassilev, V., Meledin, D., Lapkin, I., et al., A Swedish heterodyne facility instrument for the APEX telescope. 2008, A\&A, 490, 1157

Walmsley, M., Dense Cores in Molecular Clouds. 1995, in Revista Mexicana de Astronomia y Astrofisica, vol. 27, Vol. 1, Revista Mexicana de Astronomia y Astrofisica Conference Series, ed. S. Lizano \& J. M. Torrelles, $137-+$

Walsh, A. J., Burton, M. G., Hyland, A. R., \& Robinson, G., Studies of ultracompact HiI regions - II. Highresolution radio continuum and methanol maser survey. 1998, MNRAS, 301, 640

Walsh, A. J., Thorwirth, S., Beuther, H., \& Burton, M. G., Mopra line survey mapping of NGC 6334I and I(N) at 3mm. 2010, MNRAS, 404, 1396

Wang, P., Li, Z., Abel, T., \& Nakamura, F., Outflow Feedback Regulated Massive Star Formation in Parsec-Scale Cluster-Forming Clumps. 2010, ApJ, 709, 27

Watt, S. \& Mundy, L. G., Molecular Environments of Young Massive Stars: G34.26+0.15, G11.94-0.62, G33.92+0.11, and IRAS 18511+0146. 1999, ApJS, 125, 143

Wilson, T. L., Henkel, C., \& Hüttemeister, S., The detection of the $(J, K)=(18,18)$ line of $\mathrm{NH}_{3} .2006$, A\&A, 460, 533

Wilson, T. L. \& Rood, R., Abundances in the Interstellar Medium. 1994, ARA\&A, 32, 191

Wood, D. O. S. \& Churchwell, E., The morphologies and physical properties of ultracompact HiI regions. 1989, ApJS, 69, 831

Wyrowski, F., Menten, K. M., Schilke, P., et al., Revealing the environs of the remarkable southern hot core G327.3-0.6. 2006, A\&A, 454, L91

Wyrowski, F., Schilke, P., \& Walmsley, C. M., Vibrationally excited $\mathrm{HC}_{3} \mathrm{~N}$ toward hot cores. 1999, A\&A, 341, 882

Zapata, L. A., Ho, P. T. P., Schilke, P., et al., A Ring/Disk/Outflow System Associated with W51 North: A Very Massive Star in the Making. 2009a, ApJ, 698, 1422

Zapata, L. A., Leurini, S., Menten, K. M., et al., Unveiling a Compact Cluster of Massive and Young Stars in IRAS 17233-3606. 2008a, AJ, 136, 1455

Zapata, L. A., Palau, A., Ho, P. T. P., et al., Forming an early O-type star through gas accretion? 2008b, A\&A, 479, L25

Zapata, L. A., Schmid-Burgk, J., Ho, P. T. P., Rodríguez, L. F., \& Menten, K. M., Explosive Disintegration of a Massive Young Stellar System in Orion. 2009b, ApJ, 704, L45

Zapata, L. A., Schmid-Burgk, J., \& Menten, K. M., Orion KL: the hot core that is not a "hot core". 2011, A\&A, 529, A24+ 
Zapata, L. A., Tang, Y., \& Leurini, S., Extremely Large and Hot Multilayer Keplerian Disk Around the O-type Protostar W51N: The Precursors of the HCHII Regions? 2010, ApJ, 725, 1091

Zavagno, A., Anderson, L. D., Russeil, D., et al., Star formation triggered by Hir regions in our Galaxy. First results for N49 from the Herschel infrared survey of the Galactic plane. 2010, A\&A, 518, L101+

Zavagno, A., Pomarès, M., Deharveng, L., et al., Triggered star formation on the borders of the Galactic $\mathrm{H}$ ii region RCW 120. 2007, A\&A, 472, 835

Zhang, Q., Ho, P. T. P., \& Ohashi, N., Dynamical Collapse in W51 Massive Cores: CS (3-2) and $\mathrm{CH}_{3} \mathrm{CN}$ Observations. 1998, ApJ, 494, 636

Zhang, Q., Wang, Y., Pillai, T., \& Rathborne, J., Fragmentation at the Earliest Phase of Massive Star Formation. 2009, ApJ, 696, 268

Zhou, S., In search of evidence for protostellar collapse - A systematic study of line formation in low-mass dense cores. 1992, ApJ, 394, 204

Zhou, S. \& Evans, II, N. J., Kinematic Signatures of Protostellar Collapse. 1994, in Astronomical Society of the Pacific Conference Series, Vol. 65, Clouds, Cores, and Low Mass Stars, ed. D. P. Clemens \& R. Barvainis, $183-+$

Zinnecker, H. \& Yorke, H. W., Toward Understanding Massive Star Formation. 2007, ARA\&A, 45, 481

Ziurys, L. M. \& Turner, B. E., Detection of interstellar vibrationally excited HCN. 1986, ApJ, 300, L19 
Ich versichere, dass ich die von mir vorgelegte Dissertation selbständig angefertigt, die benutzten Quellen und Hilfsmittel vollständig angegeben und die Stellen der Arbeit - einschließlich Tabellen, Karten und Abbildungen -, die anderen Werken im Wortlaut oder dem Sinn nach entnommen sind, in jedem Einzelfall als Entlehnung kenntlich gemacht habe; dass diese Dissertation noch keiner anderen Fakultät oder Universität zur Prüfung vorgelegen hat; dass sie - abgesehen von unten angegebenen Teilpublikationen - noch nicht veröffentlicht worden ist sowie, dass ich eine solche Veröffentlichung vor Abschluss des Promotionsverfahrens nicht vornehmen werde.

Die Bestimmungen der Promotionsordnung sind mir bekannt. Die von mir vorgelegte Dissertation ist von Prof. Dr. Peter Schilke betreut worden.

Nachfolgend genannte Teilpublikationen liegen vor:

Rolffs, R., Schilke, P., Comito, C., et al.: Reversal of infall in SgrB2(M) revealed by Herschel/HIFI observations of HCN lines at THz frequencies. 2010, A\&A, 521, L46+

Rolffs, R., Schilke, P., Wyrowski, F., et al.: Hot HCN around young massive stars at 0.1” resolution. 2011, A\&A, 529, A76+

Rolffs, R., Schilke, P., Wyrowski, F., et al.: Structure of evolved cluster-forming regions. 2011, A\&A, 527, A68+

Rolffs, R., Schilke, P., Zhang, Q., \& Zapata, L.: Structure of the Hot Molecular Core G10.47+0.03. 2011, A\&A, eingereicht

Qin, S. L., Schilke, P., Rolffs, R., et al.: Submillimeter Continuum Observations of Sagittarius B2 at Subarcsecond Spatial Resolution. 2011, A\&A, eingereicht 